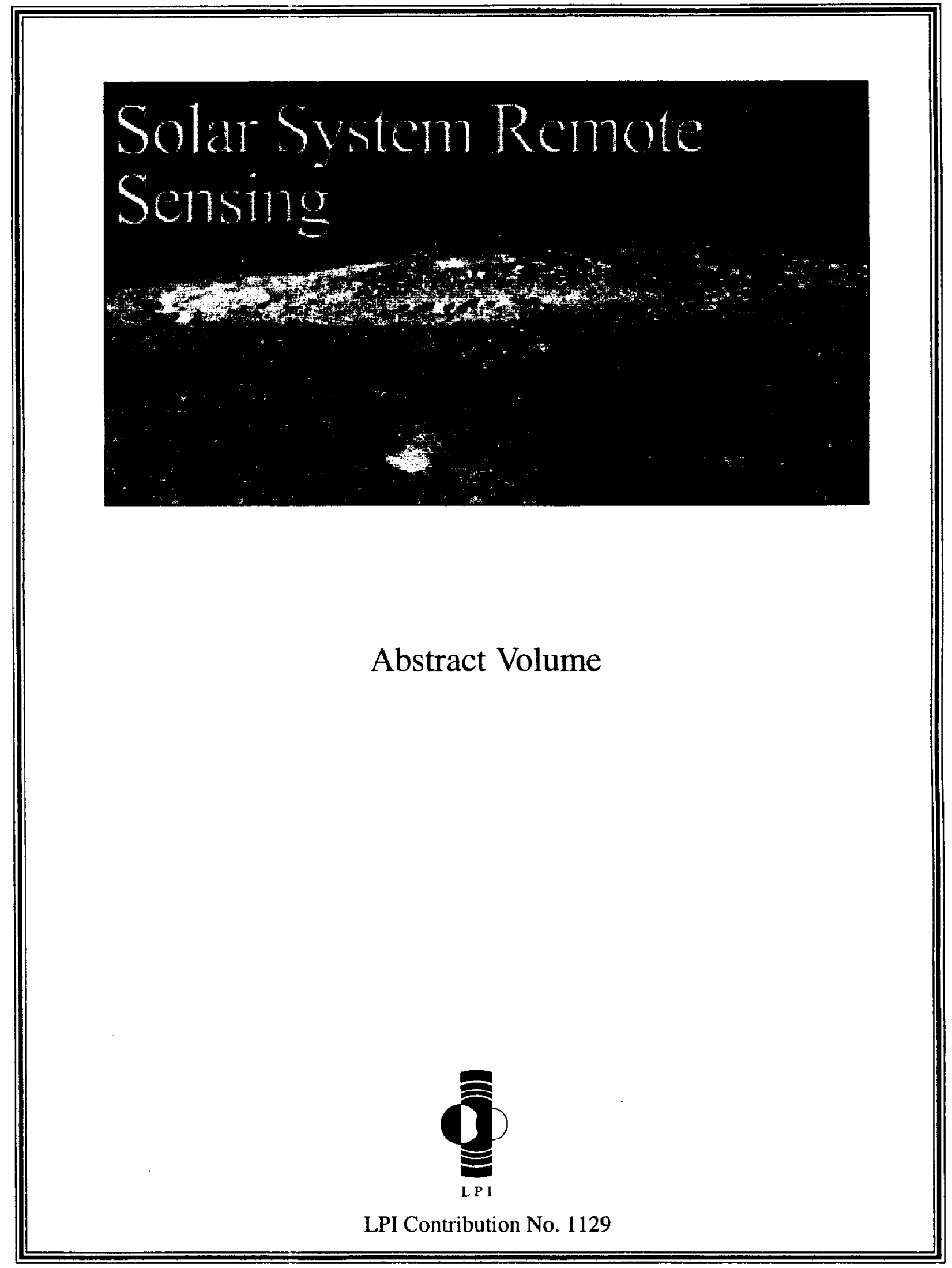




\title{
Solar System Remote Sensing
}

\author{
September 20-21, 2002
}

\section{Pittsburgh, Pennsylvania}

\author{
Sponsors \\ University of Pittsburgh \\ Lunar and Planetary Institute \\ Spectroscopy Society of Pittsburgh \\ Nilional Aeronautics and Space Administration

\begin{abstract}
Conveners
William Cassidy (University of Pittsburgh)

Deborah Domingue (Applied Physics Laboratory, Johns Hopkins University)

Rubert M. Nelson (Jet Propulsion Laboratory)
\end{abstract} \\ Scientific Organizing Committee \\ William Cassidy (University of Pittsburgh) \\ Deborah Domingue (Applied Physics Laboratory, Johns Hopkins University) \\ Rıbert M. Nelson (Jet Propulsion Laboratory) \\ Carle Pieters (Brown University) \\ Yurij Shkuratov (Kharkov Astronomical Observatory) \\ Ann Sprague (University of Arizona) \\ Joseph Veverka (Cornell University) \\ Faith Vilas (NASA Headquarters) \\ Kobert Witkowski (University of Pittsburgh)
}

Lunar and Planetary Institute 3600 Bay Area Boulevard Houston TX 77058-1113

LPI Contribution No. 1129 


\section{Compiled in 2002 by \\ LUNAR AND PLANETARY INSTITUTE}

The Institute is operated by the Universities Space Research Association under Contract No. NASW-4574 with the National Aeronautics and Space Administration.

Material in this volume may be copied without restraint for library, abstract service, education, or personal research purposes; however, republication of any paper or portion thereof requires the written permission of the authors as well as the appropriate acknowledgment of this publication.

Abstracts in this volume may be cited as

Author A. B. (2002) Title of abstract. In Solar System Remote Sensing, p. XX. LPI Contribution No. 1129, Lunar and Planetary Institute, Houston.

This volume is distributed by

ORDER DEPARTMENT

Lunar and Planetary Institute

3600 Bay Area Boulevard

Houston TX 77058-1113, USA

Phone: $281-486-2172$

Fax: 281-486-2186

E-mail: order@lpi.usra.edu

Mail order requestors will be invoiced for the cost of shipping and handling. 


\section{Preface}

This volume contains abstracts that have been accepted for presentation at the symposium on Solar System Remote Sensing, September 20-21, 2002, in

Pittsburgh, Pennsylvania.

Administration and publications support for this meeting were provided by the staff of the Publications and Program Services Departments at the Lunar and Planetary Institute. 



\section{Contents}

Effects of Rock Coating on Reflectance Spectra of Rock Samples

H. Akiyama and S. Sasaki 1

On the Distribution and Implications of Mantled and Exhumed Terrains on Mars

R. E. Arvidson, P. R. Christinsen, J. Garvin, and M. Mellon

Testing the Hapke Model for the Optical Effects of Space Weathering

D. T. Blewett and P. G. Luc' $y$ 5

Photometric Modeling of a Cometary Nucleus: Taking Hapke Modeling to the Limit

B. J. Buratti, M. D. Hicks, I. A. Soderblom, J. K. Hillier, and D. Britt

The Diverse Surface Compositions of the Galilean Satellites

R. W. Carlson

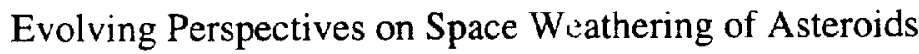

C. R. Chapman

The Nature of Space Weathering Effects on Asteroid 433 Eros

B. E. Clark, P. Lucey, and P. Helfenstein....

ANTS: Applying a New Paradigm to Lunar and Planetary Exploration

P. E. Clark, S. A. Curtis, and M. L. Rilee

Planetary Regolith Surface Analogi and Mesoscale Topography: Optimized

Determination of Hapke Parameters Using Multi-Angular Spectro-Imaging

Laboratory Data

A. Cord, P. Pinet, Y. Daydou, D. Stankevich, and Yu. Shkuratov

Toward an Improved Single-Particle Model for Large, Irregular Grains

W. M. Grundy, B. Schmitt, and S. Doute

Backscatter Effects of Particles with Perfect and Irregular Shapes:

Geometric Optics Approach

Ye. Grynko and Yu. Shkuratov

Mars Radar Observations with the Goldstone Solar System Radar

A. F. Haldemann, R. F. Jurgens, K. W. Larsen, R. E. Arvidson, and M. A. Slade ..... 23

Thermal Modeling of Planetary Regoliths

A. S. Hale and B. W. Hapk' 
A New Method for Estimating the Single Scattering Phase Functions of Regolith Grains $P$. Helfenstein.

The Lunar Phase Curve in the Near Ultraviolet

A. R. Hendrix

Ultraviolet Surface Properties of the Icy Galilean Satellites from Phase Curve Analysis A. R. Hendrix and D. L. Domingue

Earth-based Radar Speckle Displacement Interferometry to Study the Spin-Vector of Venus

I. V. Holin

Evidence for Global Radar Speckle Pattern Displacement to Measure Mercury's Obliquity and Librations

I. V. Holin

Using the Hapke Rough Reflectance Factor for Calibration of Atmospheric Emissions at Mercury and the Moon

D. M. Hunten and A. L. Sprague

Dust Coatings at the Mars Pathfinder Landing Site

J. R. Johnson, W. M. Grundy, and M. T. Lemmon

Microstructural Studies of Space Weathering Effects in Lunar Materials

L. P. Keller

Four Station Interferometric Radar Observations of Mars

$K$.W. Larsen, A. F. Haldemann, R. F. Jurgens, R. E. Arvidson, and $M$. A. Slade

A Complete First Order Hapke Model of the Near-Infrared Spectral Reflectance of the Moon

P. G. Lucey

Coherent Backscattering by Random Particulate Media in the Solar System

K. Muinonen

CRISM: Compact Reconnaissance Imaging Spectrometer for Mars on the Mars Reconnaissance Orbiter

S. Murchie, R. Arvidson, O. Barnouin-Jha, K. Beisser, J.-P. Bibring,

J. Bishop, J. Bold, T. Choo, R. T. Clancy, E. H. Darlington, D. Des Marais,

D. Fort, R. Green, J. Hayes, J. Lees, E. Malaret, D. Mehoke, R. Morris, J. Mustard, K. Peacock, M. Robinson, T. Roush, E. Schaefer, P. Silverglate, M. Smith, P. Thompson, and B. Tossman. 
Small Particles and Cemented Textures: Two Challenges for

Remote Compositional Analysis

J. F. Mustard

The Opposition Effect: A Very Unusual Case

R. M. Nelson 53

Space Weathering Processes on Mercury

S. K. Noble and C. M. Pieters 55

New Estimates of Lunar Opposition Spike Characteristics with Clementine Data

V. V. Omelchenko, V. G. Kavdash, and Yu. G. Shkuratov 57

Groundbased Radar Investigations of Asteroids and Planetary Satellites

S. J. Ostro 59

Size-dependent Scattering Propertics of Planetary Regolith Analogs

J. L. Piatek, B. Hapke, R. M. Nelson, A. S. Hale, and W. D. Smythe

Lessons Learned from Lunar Exploration: The Moon Continues to Surprise Us

C. M. Pieters

Intercontinental Bistatic Radar Tes1 Observation of Asteroid 1998 WT24

S. Righini, S. Poppi, S. Montebugnoli, M. Di Martino, L. Saba, M. Delbò,

S. Ostro, J. Monari, M. Poloni, A. Orlati, and A. Zaitsev 65

Compositional Remote Sensing of Icy Planets and Satellites Beyond Jupiter

T. L. Roush

Simulation of Space Weathering by Nanosecond Pulse Laser Irradiation:

Spectral Change and TEM-ESR Confirmation of Nanophase Iron Particles

S. Sasaki, E. Kurahashi, K. Nakamura, T. Hiroi, and C. Yamanaka

Dependence of the Spectral BRDF of Sulfur Surfaces with Material Texture:

Laboratory Measurements with the Spectrophoto-Goniometer at LPG

B. Schmitt, N. Bonnefoy, O. Brissaud, and S. Douté

Remote Sensing Analysis of the Terra Meridiani Region, Mars

F. P. Seelos IV and R. E. Arvidson

Initial Results from the Bloomsbur: University Goniometer Laboratory

$M$. K. Shepard 
Interpreting Photometry of Planetary Regoliths: Progress and Problems as Seen from Kharkov

Yu. G. Shkuratov

Mercury's Surface Composition from Surface Spectroscopy

A. L. Sprague

Light Scatter by Particulate Surfaces with Different Topography

D. Stankevich, Yu. Shkuratov, P. Pinet, and A. Cord...

Combining the MGM and Hapke Theory: Two Models are Better than One

J. M. Sunshine, B. E. Clark, and P. G. Lucey.

Origin of Surface-correlated and Agglutinitic Nanophase $\mathrm{Fe}^{0}$ : A Bedtime Story for Bruce

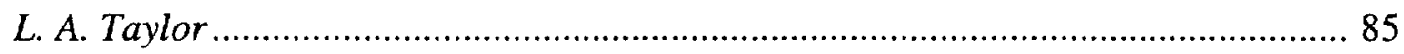

Unmixing the Lunar Surface

S. Tompkins.

Hubble Space Telescope Photometry of Mimas, Enceladus, Tethys, Dione, and Rhea

A. Verbiscer, R. French, and C. McGhee

End Member Analyses of Spaceborne Thermal Infrared Data of Meteor Crater, Arizona and Application to Future Mars Data Sets

S. P. Wright and M. S. Ramsey

An Automated Spectral Deconvolution Algorithm: Application to Thermal Infrared Studies of Earth and Mars

N. V. Zorn and M. S. Ramsey

Negative Polarization of Light Scattered by Cometary Dust and Planetary Regolith:

Two Different Mechanisms

E. S. Zubko and Yu. G. Shkuratov 
EFFECTS OF ROCK COATING ON REFLECTANCE SPECTRA OF ROCK SAMPLES. H. Akiyama ${ }^{1}$ and S. Sasaki ${ }^{2}{ }^{1}$ Nishimatsu Construction C1., Ltd. 2-4-10-202 Fukami Nishi Yamato-city Kanagawa-pref. 242-0018 Japan akiyama@dream.big.jp, ${ }^{2}$ Dept.Earth \& Planetary Sci., Univ. Tokyo, Tokyo 113-0033 JAPAN sho@eps.s.utokyo.ac.jp.

Introduction: Optical theory of "rock coating (dust film type)" is important, because rock surface on planetary bodies is frequently covired with another materials such as dust and regolith. Rock coating is defined as the state that the surface if the rock is covered by material of differing optical properties [1].

Hapke [2] applied a two-layer model to the rock coating. However, validity of the twi-layer model has not been discussed enough. Hapks: compared measured values [3] with his theoretical values only at a specific wavelength $(750 \mathrm{~nm})$. Thes: is no guarantee that measured value agrees well with the theoretical value in other wavelengths. We tried a different method to match the measured value for wide range of wavelength.

The "adding method" is used in the studies of vegetation canopy and of solar emission. In this method, multiple scattering between coating layer and rock surface is calculated and reflectance of coated rock surface is estimated. We here applied the adding method to the rock coating.

Experimental Method: As for dust film-type rock coating, it is important to measure the optical properties of the film and the reflectance if the coated surface. In this paper, olivine powders were used as a material of dust film. We scattered powders thinly and homogeneously on the surface of the rock, and simulated rock coating (Fig.-1). Thickness of the olivine layer at each survey point was measured by a laser displacement gage, and the reflectance of each survey point was measured by the bidirectional spectrometer.

Moreover, the reflectance and transmittance of the olivine layers were measured using an integrating sphere, since the reflectance and trinsmittance of the covered layer should be known in the adding method.

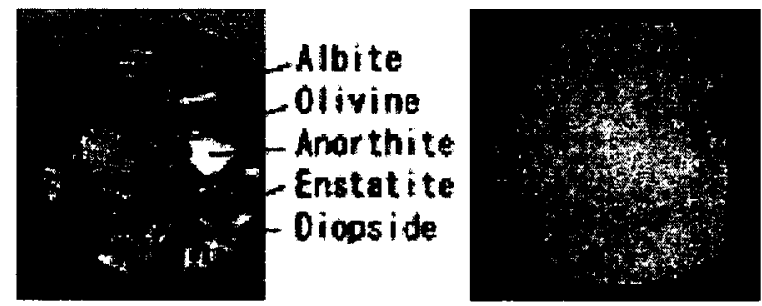

Fig.-1 Mineral palette (simulate rock surface) and Rock Coated surface
Results and Discussion: We compared the measured values and theoretical values by Hapke's. Comparisons between the measured values and both theoretical values for the optical depth are shown in Fig.-2. When optical depth is large, the measured values agree well with both theoretical values. However, when optical depth is small, the Hapke's values gives large disagreement approaching at most $70 \%$. On the other hand, disagreements of the new theoretical values remain less than $10 \%$.

In Hapke two-layer model, the optical properties of each coating layer are estimated implicitly assuming the layer thickness is sufficiently large for multiple scattering. If the layer is optically thin, the theory cannot be applied. Multiple scattering is not expected when the layer's optical depth is less than 1 . As for Fig.-2, when optical depth is smaller than 1, Hapke theoretical reflectance values disagrees from the measurement value greatly.

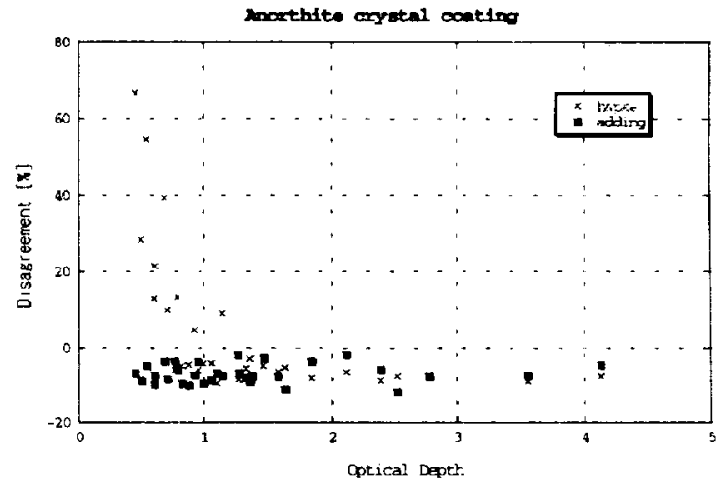

Fig.-2 Disagreement of measured reflectance values from theoretical values.

Doubling Method: Using a so-called doubling method, we can calculate reflectance and transmittance of any thickness of layer if we have reflectance and transmittance data of one optically-thin layer. However, it is not clear that doubling method can be applied to the rock coating. We performed measurements to confirm the applicability of the doubling method. Using optical properties of spherical glass beads and irregularly-shaped olivine powder, theoretical values of the film for thickness of approximately $0.4 \mathrm{~mm}$ was calculated from the measured values of $0.2 \mathrm{~mm}$ thickness. Fig.-3 shows the result. The disagreement of the 
measured values and the theoretical values are less than $20 \%$ when the particle size is under $90 \mu \mathrm{m}$ as for about $0.5 \mathrm{~mm}$ thickness layer. The disagreement is larger than $40 \%$ in the particle size over $125 \mu \mathrm{m}$.

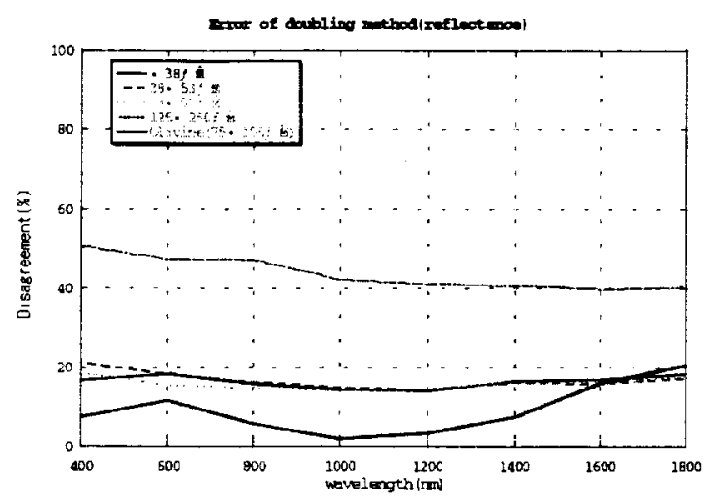

Fig.-3 Disagreement of reflectance between theoretical $0.4 \mathrm{~mm}$ thickness values by the doubling method and measured $0.4 \mathrm{~mm}$ thickness values.

Application: There are various applications of the new rock coating theory. MARS Pathfinder, which landed and surveyed MARS surface, observed two types of rock coating. First, IMP (Imager for MARS Pathfinder) observed colored rock surface. Some rocks, like "Yogi", they have two colors; half side of them is bluish gray and half side of them is red. Bluish gray side is original rock surface color, and red is the color of the oxidized iron contained in the circumference regolith [4]. Using these surfaces spectral data and $\mathrm{Fe}_{2} \mathrm{O}_{3}$ spectral data, we estimated the thickness of this coating layer. It can be estimated that that thickness is about $0.08 \mathrm{~mm}$ from this experiment if the material, which covers Barnacle Bill, is supposed to be the $\mathrm{Fe}_{2} \mathrm{O}_{3}$ particles of $0.1 \mu \mathrm{m}$ size.

Second, MARS Pathfinder had two radiative calibration targets (RCTs). Their reflectance were changing because airborne dust coated their surfaces. IMP observed change of target reflectance. We used these data, and estimated the thickness of the air-borne dustcoating layer. As for this estimate, we used Hematite reflectance to airborne dust reflectance. Martian Sol 10 data agrees well with $0.13 \mathrm{~mm}$ thickness theoretical data, and Sol 20 and Sol 35 data agree well with $0.25 \mathrm{~mm}$ thickness theoretical data. And Sol 52 data agrees well with 0.50 thickness theoretical data.

However actually, the covered particle size and their chemical compositions are different. This discussion is only a preliminary examination.

References: [1] R.Dorn (1998) "Rock Coating", Elsevier, Amsterdam. [2] B. Hapke (1993) "Theory of reflectance and emittance spectroscopy", University Press, Cambridge. [3] Wells, E. et al. (1984) lCARUS, 58, 331-338. [4] Douglas L. Smith (1997) Engineering \& Science, 3, 8-17. 
On the Distribution and Implications of Mantled and Exhumed Terrains on Mars. R. E. Arvidson', P. R. Christensen ${ }^{2}$, J. Garvin ${ }^{3}$, M. Mellon ${ }^{4}$, 'D partment of Earth and Planetary Sciences, McDonnell Center for the Space Sciences, Washington University, St. Louis, MO, 63130 (arvidson@wunder.wustl.edu), ${ }^{2}$ Arizona State University, Phoenix, AZ, ${ }^{3}$ NASA Goddard Space Flight Center, Greenbelt, MD, ${ }^{4}$ University of Colorado, Boulder, CO.

Introduction: The intent of this paper is to show the relationships for Mars among althedo, thermal inertia, roughness inferred from MOLA pulse width spread data, and geology inferred from photogeological analyses. Mapping of surface units using these parameters and approaches, in combination with analysis of hyperspectral image data from ISM, TES, OMEGA, and CRISM observations, will maximizt: our understanding of the distribution and nature of surtace units on the red planet. Results will directly impact the selection of landing sites that exhibit geological records needed to understand planetary habitability.

Data Sets and Approach: Our approach is to compile and examine a global-scale image cube consisting of MOC WA Geodesy Canpaign mosaics [1], MOLA-based elevation [2], MOLA pulse width data that have been corrected for regional scale tilts (CPW) [3], bolometric albedo (over solar wavelengths) from TES observations [4], and thermal inertias modeled from TES emission data [5]. These observations were examined together (e.g., Fig. 1) to infer surface properties and to map geologic units, emphasizing the nature and extent of mantling of preexisting terrains. The work is preparatory to establishing units for analysis of TES spectral emission data, ISM spectral reflectance data, and upcoming OMEGA and CRISM observations. In addition, crater counts were used to help understand the relationships between surface properties, morphology, and the extent of mantling.

Results: Previous work has shown that there is a negative correlation on a global basis between albedo and thermal inertia [e.g., 5]. In particular the Tharsis Plateau, Elysium region, and Arabia Terra have high albedoes and low thermal inertias, consistent with dust mantles. The classical dark areas have high inertias and there is an intermediate surface that has been interpreted as due to exposures of weakly indurated soil deposits of the type documented at the Viking landing sites.

We add a number of new points to this type of analysis:

- The dust and duricrust surfaces have elevated $\mathrm{CPW}$ values relative to classical dark areas. Further, examination of MOC WA and NA images for the dust covered areas in Arabia Terra shows that the deposits are tens of meters in thickness in most cases, thinning to approximately ten meters at the edges. Crater size frequency data for the mantled areas are consistent with this interpretation and with a steady accumulation of dust at a rate of a fraction of a micrometer per year. Arabia has been accumulating dust for billions of years. Similar trends are emerging for dust-covered areas on the Tharsis Plateau and within Elysium.

- A number of units on the Tharsis Plateau and within the Elysium region exhibit very high $\mathrm{CPW}$ values along with intermediate albedoes and low inertias. These materials clearly correspond to wind eroded ash deposits.

- Dark, high thermal inertia terrains have low CPW values as compared to the dust mantled areas. This is an interesting pattern that presumably is associated with reworking of surfaces by aeolian activity. We offer two possibilities: local reworking of dust into bedforms and surfaces or beveling and grading of the dark areas by wind.

- High inertia and CPW surfaces can also be found locally within craters, ejecta deposits, and within fracture and valley systems, particularly in Valles Marineris.

- Exhumed terrains can be found at the margins of the dust mantles, e.g., in Terra Meridiani. In this region a widespread set of exhumed layered deposits can be found, and each major horizon mapped from MOC WA and NA images exhibits unique albedo, color, inertia, CPW, and spectral emissivity properties. This includes the hematite deposit mapped from TES data [6] and the underlying etched unit that has morphologic patterns similar to the young platy ridged pahoehoe flows mapped in the Athabasca region. On the other hand, dust mantles show uniform spectral signatures [4].

Implications: We restrict implications to those associated with targeting OMEGA and CRISM data for high spectral and spatial resolution mapping. This targeting is for spectral analyses of the sites to understand planetary habitability and to help select landing sites for surface exploration of terrains that would provide strong evidence of past climatic or hydrothermal conditions and thus habitability. The bottom line is that the dust mantles should be avoided since these regions have accumulated up to tens of meters of aeolian cover. Careful mapping of exhumed or non-mantled terrains is 
needed to find places that preserve and expose evidence of ancient aqueous conditions. These targets will form the core of the list for detailed hyperspectral mapping.

References: [1] Caplinger and Malin, (2001) ,JGR, 106, 23595-23606. [2] Smith et al., (2001), JGR, 106,
23689-23722. [3] Garvin et al., (1999), GRL, 26, 381 384. [4] Christensen et al. (2001), JGR, 106, 23823 23871. [5] Mellon, M. et al., (2001), Icarus, 148, 437 455. [6] Christensen et al. (2001), JGR, 106, 2387323886.
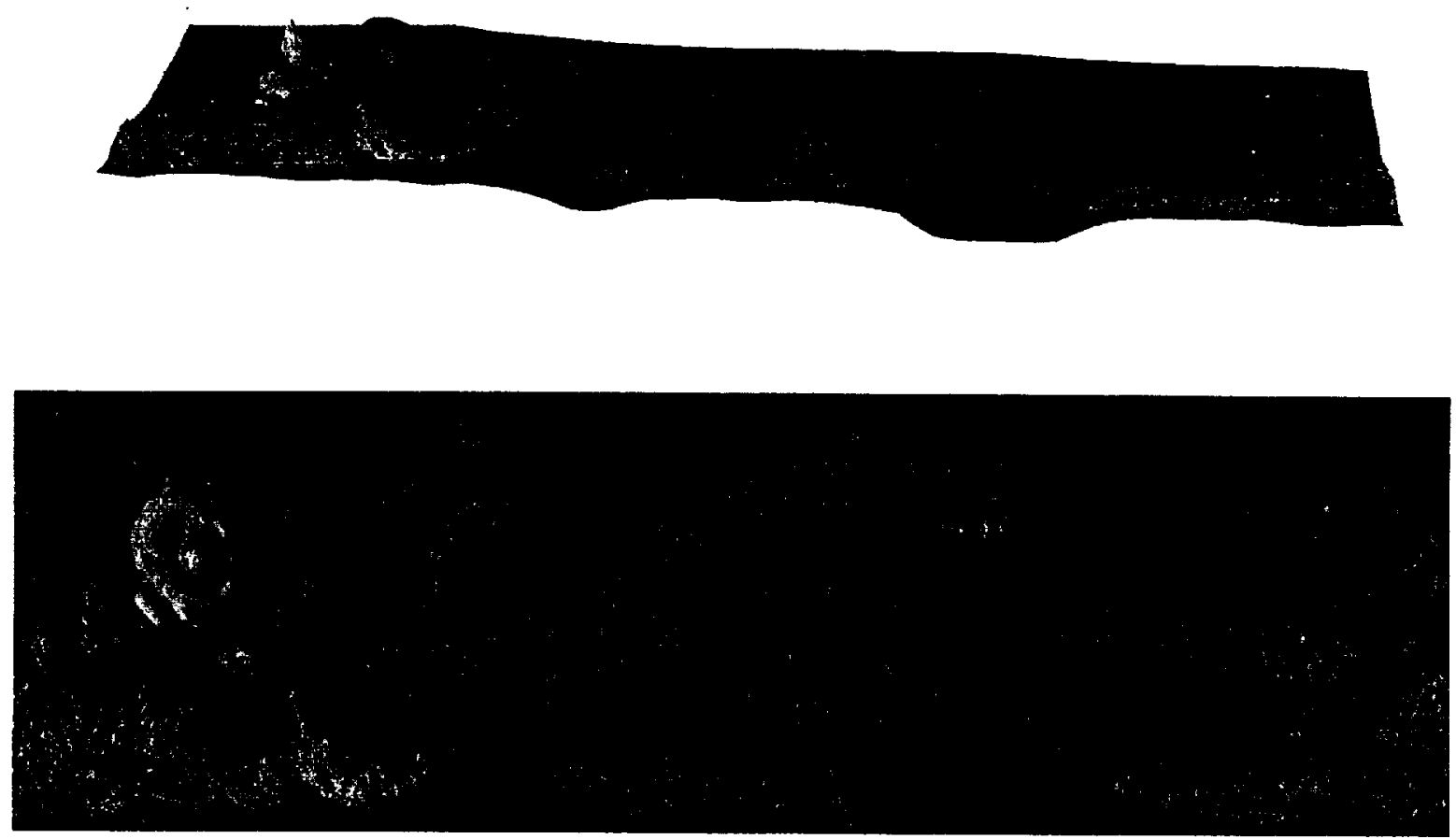

Figure 1: Perspective view (top) and nadir view (bottom) covering all longitudes and from $50^{\circ} \mathrm{N}$ to $60^{\circ} \mathrm{S}$ in latitude. Red color is modulated by TES-based bolometric albedo [4], green by MOLA topography-corrected pulse widths [2], and blue by TES-based thermal inertia [5]. Colors have been overlain on a shaded relied map generated from MOLA elevation data (2). The prime meridian is located in the middle of the frame. Relief exaggeration for the perspective view is 200:1. Orange areas correspond to regional-scale dust deposits that are bright, have low inertias, and are rough. These include Arabia Terra, the triangularly-shaped area in the middle, top part of the map. Yellow areas correspond to wind-eroded volcanic ash deposits that have intermediate albedoes, low inertias, and are very rough. Blue areas are the classical dark areas with high inertias and low roughness values. Purple regions have intermediate brightness and thermal inertia, roughness values similar to regional-scale dust deposits, and have been interpreted as consisting of duricrust. 
TESTING THE HAPKE MODEL FOR THE OPTICAL EFFECTS OF SPACE WEATHERING. D. T. Blewett ${ }^{1}$ and P. G. Lucey ${ }^{2},{ }^{1}$ NovaSol, 1:00 Alakea St., 23rd Floor, Honolulu, HI 96813 (dave@nova-sol.com), ${ }^{2}$ Hawaii Institute of Geophyiscs \& Planesılogy, Univ. of Hawaii, 2525 Correa Rd., Honolulu, HI 96822 (lucey@higp.hawaii.edu).

Introduction: In the Apollo er., Hapke and colleagues used simulation experiments and theoretical considerations to argue for the importance of vaporphase coatings in causing lunar solls to darken and redden with age [1]. During the $199($ s, direct detection of vapor-deposited iron on actual lunar soil grains came from the work of Keller and associates [2]. These workers discovered that mosi soil grains have coatings, some of which differ from the interior composition. The morphology of the coatings is such that they must have condensed from a vapor. The coatings and grain rims typically contain akundant submicroscopic iron particles (SMFe). While SMFe is largely responsible for the reddening of lunar soil, Hapke's work suggests that it alone does not account for the changes accompanying soil maturity. For example, Hapke [3] found that the inclusion of dark glass was required in addition to submicroscopic iron to account for weathering trends. Using a ditferent formalism, Shkuratov et al. [4] emphasized the importance of known particle size evolution in maluration modeling to match observed space weathering Irends. Therefore Hapke's ground-breaking work must be followed up to show how the model glass content and composition and grain size evolution required to fit ohserved data compare to measured soil properties.

Spectral Modeling: We have developed computer code to produce model spectra and model fits to laboratory or telescopic spectra using the equations in [3]. The program uses 11 parameters: the abundances of olivine, orthopyroxene, clinopyroxi'ne, anorthite, ilmenite, glass, large-grained metal, and SMFe, the grainsize, the $\mathrm{Mg \#}$, and the $\mathrm{FeO}$ content of the glass. Figure 1 shows a telescopic near-infrared reflectance spectrum of Aratus, a $10-\mathrm{km}$ diameter highland crater located in the Hadley-Apennine reçion of the Moon [5]. The test fit shown in the plot for Aratus was done

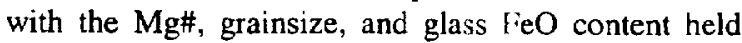
constant. The fit parameters were: of, $11 \%$; opx, $17 \%$; cpx, $4 \%$; an, $52 \%$; ilm, $2 \%$; glass, $14 \%$, large-grain $\mathrm{Fe}$, $0 \%$, and SMFe, $0.08 \%$. This produces an excellent fit to the original spectrum. We then trok this composition and held it constant except for the SMFe content, which we varied in equal steps from 0.005 to 0.23 wt.\%. This produces a simulated space-weathering trend. The results are shown in Figure 1. The model spectrum with very little SMFe (top s.jectrum in Figure 1) is similar to spectra of powdered lunar rocks, with strong absorption bands and a flat continuum. As optical maturation proceeds, the overall albedo drops, the continuum reddens, and the absorption bands are attenuated.

We have undertaken a study to use the Hapke model, including grain size and glass content evolution (representing accumulating agglutinate glass), to fit the Apollo 16 soil maturity series of Fischer and Pieters [6] and compare the model results to the known correlation of glass content, particle size and SMFe content. The Apollo 16 samples have nearly constant composition and mineralogy, but differ in maturity, i.e., they have variations in grainsize and the abundance agglutinitic glass and SMFe. Bidirectional reflectance spectra for Apollo 16 samples 60051, 61141, 61161, 61221, 61241 , and 67461 were retrieved from the RELAB web site at Brown University [7]. The laboratory spectra and model fits are shown in Figure 2. As expected, the amount of SMFe in the model fits increases with the maturity of the samples.

We plan to compare the model predictions of glass content, particle size and SMFe content to the actual measured values. Ultimately our goal is to couple these parameters in a systematic and physically realistic way, and apply these systematics to other highland and mare soils in the RELAB, telescopic and Clementine database. We also seek to understand the compositional controls on the OMAT optical maturity parameter [8] that has been used to remotely map relative variations in soil maturity.

Acknowlegement: DTB thanks Bruce Hapke for serving as a mentor and teacher early in his career.

References: [1] Cassidy, W. and B. Hapke, Icarus 25, 371-383, 1975; Hapke, B., Moon 7, 342-355, 1973; Hapke, B., Phys. Earth Planet. Inter. 15, 264274, 1977; Hapke, B. et al., Moon 13, 339-354, 1975. [2] Keller, L. P. and D. S. McKay, Science 261, 13051307, 1993; Keller, L. P. and D. S. McKay, Geochim. Cosmochim. Acta 61, 2331-2340, 1997; Keller, L. P. et al., in Workshop on New Views of the Moon, p. 4445, 1998. Wentworth, S. J. et al., Meteorit. Planet. Sci. 34, 593-603, 1999. [3] Hapke, B., J. Geophys. Res. 106, 10,039-10,074, 2001. [4] Shkuratov, Y. G. et al. Icarus 137, 222-234, 1999. [5] Blewett, D. T. and B. R. Hawke, Meteorit. Planet. Sci. 36, no. 5, 701-730, 2001. [6] Fischer, E. M. and C. M. Pieters, Icarus 111 , 475-488, 1994; Fischer, E. M. and C. M. Pieters, $J$. 
Geophys. Res. 101, no. E1, 2225-2234, 1996. [7] http://If314rlds.geo.brown.edu/. [8] Lucey, P. G. et al., J. Geophys. Res. 105, no. E8, 20,377-20,386, 2000; Grier, J. A. et al., J. Geophys. Res. 106, no. E12, $32,847-32,862,2001$.

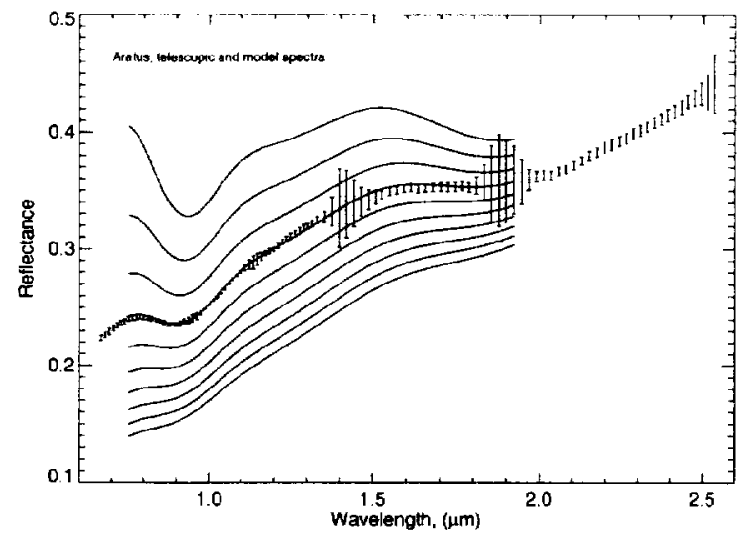

Figure 1. Telescopic spectrum (points with error bars) and model spectra (solid lines) for the crater Aratus. From top to bottom, the model spectra represent a space-weathering trend produced by increasing the amount of SMFe, with other parameters held constant. Telescopic spectrum is scaled to the Clementine 0.75 $\mu \mathrm{m}$ albedo of the area covered by the spectrometer aperture. Model plots were done in the range 0.75 $2.0 \mu \mathrm{m}$ because of limitations in lab data for pure minerals in the UV, and uncertainties in the optical constants of Fe at long wavelengths.

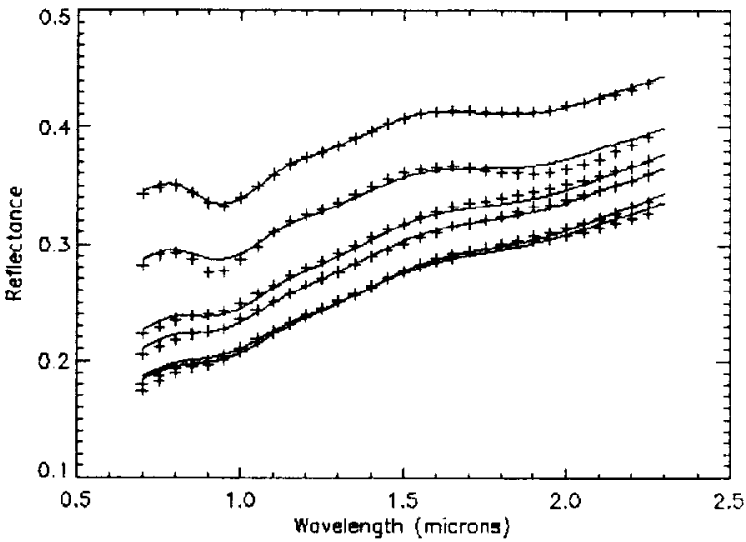

Figure 2. Reflectance spectra for six Apollo 16 soil samples with similar compositions but differing exposure degree (maturity) are shown with the symbol $(+)$. Model fits for each spectrum computed from Hapke's equations are shown as solid lines. 
PHOTOMETRIC MODELING OF A COMETARY NUCLEUS: TAKING HAPKE MODELING TO THE LIMIT. B. J. Buratti (IPL), M. D. Hicks (JPL), L. Soderblom (USGS), J. Hillier (Grays Harbor College), D. Britt (U. Tenn.)

In the past two decades, photonietric models developed by Bruce Hapke have been fit to a wide range of bodies in the Solar System: The Moon, Mercury, several asteroids. and many icy and rocky satellites. These models have enabled comparative descriptions of the physical attributes of planetary surfaces, including macroscopic roughness, particle size and sizedistribution, the single scattering albedo, and the compaction state of the optically active portion of the regolith. One challenging type of body to observe and model, a cometary nucleus, awaited the first space based mission to obtain images unobscured by coma.

The NASA-JPL Deep Space 1 Mission (DS1) encountered the short-period Jupiter-family comet 19/P Borrelly on September 22, 2001, about 8 days after perihelion. Prıor to its closest approach of $2171 \mathrm{~km}$, the remote-sensing package on the spacecraft obtained $25 \mathrm{CCD}$ images of the comet, representing the first closeup, unobscured view of a comet's nucleus (1). At closest approach, corresponding to a resolution of 47 meters per pixel. the intensity of the coma was less than $1 \%$ of that of the nucleus. An unprecented range (If high solar phase angles (52-89 degrees), vie wing geometries that are in general attainable only when a comet is active, enabled the first quantitative and disk-resolved modeling of surface photometric physical parameters.

The geometric albedo of Borrelly is $0.029 \pm$ 0.006 , comparable to the dark hemisphere of
Iapetus, the lowest albedo C-type asteroids, and the Uranian rings. The Bond albedo, $0.009 \pm$ 0.002 , is lower than that of any Solar System object measured. Such a low value may enhance the heating of the nucleus and sublimation of volatiles, which in turn causes the albedo to decrease even further. A map of normal reflectance of Borrelly shows variations far greater than those seen on asteroids. The two main terrain types, smooth and mottled (2), exhibit mean normal reflectances of 0.03 and 0.022 .

Borrelly's physical photometric parameters are typical of other small dark bodies. The nucleus exhibits significant variations in macroscopic roughness, with the oldest, darkest terrain being slightly smoother. This result suggests the infilling of low-lying areas with dust and particles that have not been able to leave the comet. The surface of the comet is backscattering, but there are substantial variations in the single particle phase function. One region scatters isotropically (like cometary dust), suggesting that its regolith is controlled by native dust rather than by meteoritic bombardment.

Work performed at NASA's Jet Propulsion Laboratory, California Institute of Technology.

(1) Soderblom, L. et al. (2002). Science 296, 1087.

(2) Britt, D. et al. (2002). LPSC Abstract 1686. 

THE DIVERSE SURFACE COMPOSITIONS OF THE GALILEAN SATELLITES. R. W. Carlson,' Jet Propulsion Laboratory, Califormia Institıte of Technology, Pasadena, CA 91109 (rcarlson@lively.jpl.nasa.gov).

The galilean satellites represent a diversc collection, ranging from the volcanic moon Io, with a surlace that is changing yearly, to Callisto, with a dark, ancient surface overlying ice. The composition of these surfaces are al is quite different due to a variety of processes and influences, including tidal heating, radiolysis, gardening, a magnetic fisld (Ganymede), and meteoritic infall.

Io's surface contains large quantities of sulfur dioxide $\left(\mathrm{SO}_{2}\right)$ and colorful sulfur allotropes, both originating in plumes and flows from the tidally driven volcanoes. A broad, $1-\mu \mathrm{m}$ band is found at high latitudes and may be itue to absorption by long-chain sulfur polymers produced by $\mathrm{SO}_{2}$ radiolysis, although iron and iron sulfide compounds are candidates. An unidentified $3.15 \mu \mathrm{m}$ absorber is equatorially distributed while a $4.62 \mu \mathrm{m}$ band, perhaps due to a sulfate compound, exhibits a non-uniform distribution. Hot spots are generally dark, and some exhibit negative reflectance slopes (i.e., blue). The composition of these lavas has not been established spectroscopically, but the high temperatures of some volcanoes suggest ultramafic silicates or perhaps more refractory material such as oxides.

Europa's icy surface contains the radiclytic product hydrogen peroxide $\left(\mathrm{H}_{2} \mathrm{O}_{2}\right)$. This molecule is rapidly destroyed by solar ultraviolet radiation, so replenishnent of $\mathrm{H}_{2} \mathrm{O}_{2}$ by highenergy charged particle impact must be occurring continuously. The same process may produce alınospheric and surficial $\mathrm{O}_{2}$ and the hydrated compound thit was found on Europa, suggested to be hydrated sulfuric acid. The hydrate is observed to be associated with visually dark material, whose spectra resemble sulfur polymers, and with surficial $\mathrm{SO}_{2}$. $\mathrm{A}$ radiolytic sulfur cycle continuously reates and destroys these compounds, and their observed relative abundance agrees with predictions based on labcratory radiolysis experiments. The equilibration time for this cycle is $\sim 100$ years. Sources of sulfurous material include Iogenic plasma implantation, preferentially deposited in the trailing hemisphere where the hydrated acid, visually dark material, and
$\mathrm{SO}_{2}$ are indeed found to concentrated. Thermal processes such as diapiric heating, accompanied by $\mathrm{H}_{2} \mathrm{O}$ sublimation, can further concentrate the sulfur compounds and produce the variegated surface patterns, while gardening and asynchronous rotation modify the global patterns. An alternative suggestion for Europa's hydrate is hydrated sulfate salts from an internal ocean, but the radiolytic lifetime of these compounds at the surface is $<4000$ years so they would be rapidly assimilated into the $\mathrm{H}_{2} \mathrm{SO}_{4}, \mathrm{SO}_{2}$, and $\mathrm{S}_{x}$ cycle. NIMS spectra are best matched with sulfuric acid hydrate. Surficial carbon dioxide is also observed on the leading hemisphere of Europa. This $\mathrm{CO}_{2}$ may arise from the radiolysis of carbonaceous meteoritic material that is deposited preferentially on the leading hemisphere or it may be an outgassing product.

Ganymede and Callisto are both covered by a dark deposit overlying ice, the latter exposed on ridges, slopes, and within craters. Both satellites show similar spectroscopic features, generally more pronounced in Callisto's spectra. $\mathrm{CO}_{2}$ is observed to be concentrated on Callisto's trailing hemisphere, suggestive of magnetospheric influence, in particular radiolysis. A corresponding $\mathrm{CO}_{2}$ atmosphere is found on Callisto. The atmosphere is rapidly depleted by ionization and sweeping, so there must be a continuous source of $\mathrm{CO}_{2}$ such as radiolysis or outgassing. A band at $3.88 \mu \mathrm{m}$ has been suggested to be an SH stretch band, but radiolyticallyproduced $\mathrm{H}_{2} \mathrm{CO}_{3}$ provides an equally good fit. A $4.57-\mu \mathrm{m}$ band has been suggested to be from a $\mathrm{CN}$ stretch transition but the shape and position is better fit by carbon suboxide $\left(\mathrm{C}_{3} \mathrm{O}_{2}\right)$, a radiolysis product of $\mathrm{CO}_{2}$. Sulfur dioxide has been suggested as a surface constituent on both Ganymede and Callisto based on a 4- $\mu \mathrm{m}$ infrared feature. However, the corresponding feature is not observed on Europa even though an $\mathrm{SO}_{2}$ ultraviolet feature is present in Europa's trailing side spectra. Ganymede and Callisto's $4-\mu \mathrm{m}$ feature may represent something other than $\mathrm{SO}_{2}$ 
EVOLVING PERSPECTIVES ON SPACE WEATHERING OF ASTEROIDS. Clark R. Chapman, Southwest Research Institute, Suit: 400, 1050 Walnut St., Boulder CO 80302.

Efforts to measure the color: of asteroids date back at least as far as the $19.20 \mathrm{~s}$, when Bobrovnikoff's spectroscopy actually yielded Vesta's rotation period based on cyclical changes in its reflectance spectruni. There were several efforts in the 1940 s and 1950 s to compare asteroid colors with laboratory measurements of meteorite colors. They proved inconclusive for several reasons, including (a) the blandness of mineral spectra in the shortwavelength bands commonly used in astronomical photometry and (b) poor preparation of often terrestrially weathered meteorites.

In the early 1970 s, both laboratory measurements of properly prepared meteorite samples and astronomical observations of visible and near-IR asteroid spectra had advanced and were sufficient to address the fundamental questions concerning asteroid mineralogy and relationship to meteorites. Two basic approaches were employed: (a) attempts to assess asteroid mineralogy from first principles, based on absorption band centers for instance, and (b) spectral matching, in which it was simply assumed that if an asteroid spectrum resembled the spectrum of a particular class of meteorite it could be inferred that the two had similar mıneralogy, even if the specific spectral features were not understood or were not unique.

Application of both approaches yielded a few successes, particularly in associaing Vesta with basaltic achondritic meteorites, but they generally revealed an unexpected disparity: despite sharing some spectral similarities, the most common asteroid type (not corrected for observational selection) could not be reconciled with the most common meteorite type (again, not corrected for atmospheric filtering processes). Thus emerged the conundrum in which S-type asteroids apparently yielded few meteorites while ordinary chondrites (OCs) had essentially no observable parent bodies in the asteroid belt.

Several prominent cosmochemists assumed that the S-types must be associated with OCs and that the disparities must be due to unknown technical issues associated with the remote- sensing techniques or interpretations. But asteroid specialists (including myself) reached a nearly unanimous consensus from the late $70 \mathrm{~s}$ through the early 90s that it was not "scientific" to adopt unknown space weathering processes to resolve the discrepancy. They turned instead to adopting equally unknown, very selective dynamical processes (which had little basis in the published literature of asteroid physics concerning dynamical or collisional processes) to derive OCs from small, rare, generally unseen asteroids while excluding meteorite delivery from the abundant S- type asteroids, which even dominated the Near-Earth Asteroid (NEA) population. This attitude of asteroid specialists was very frustrating for cosmochemists since the degree of space weathering required to resolve the conundrum was in fact modest compared with the extraordinary (if not specifically understood) space weathering effects that clearly were responsible for the dramatic differences between telescopic reflectance spectra of the lunar surface compared with laboratory measurements of lunar samples.

Once again, despite the in-your-face evidence for lunar space weathering, most specialists refused to accept any extrapolation to the asteroids until the actual physical processes responsible for lunar space weathering were identified. In the mid-90s, several types of asteroid observations (e.g., Galileo studies of Gaspra and Ida and spectral studies of small NEAs) demonstrated that space weathering processes (of some unknown sort) must be occurring on asteroids. Simultaneously, laboratory studies finally identified the predominant causes for lunar space weathering as well as showed that micrometeorite "zapping" of asteroidal minerals can modify their spectra in the sense that S-types differ from OCs. Finally, in the last few years, advances in asteroid dynamics (primarily due to enhanced capabilities of numerical simulations) have demonstrated that the combination of minor resonances and the Yarkovsky Effect necessarily leads to general, representative sampling (rather than highly selective sampling) from most of the 
EVOLVING PERSPECTIVES ON SPACE WEATHERING OF ASTEROIDS. C. R. Chapman

inner half of the main asteroid belt. So there can no longer be any doubt about the association of OCs with many S-type asteroids and the reality of space weathering processes (probably associated with the solar wind and micrometeorite bombardment) as the dominant cause of the discrepancies.

In retrospect, one wonders why it took more than a quarter of a century for the early advocacy of space weathering (by Bruce Hapke, among others) to gain wide acceptance. Somehow, the demand for explicit physical "proof" of specific space weathering processes (even as NASA refused to fund the required research on space weathering) delayed for three decades the acceptance of what we now realize is correct. Such attitudes can now be seen to have been intellectually unsupportable: the asteroid spectroscopists generally refused to take seriously the evidence from fields outside their discipline, specifically physicists, whose evolving understanding of collisional and dynamical processes never supported the extreme selectivities on which the asteroid remote-sensing community relied.

Asteroid science was similarly led astray for a few years (in the late 60s and early 70 s), by analogous attitudes on the part of dynamicists, such as George Wetherill, who refused to believe that any meteorites could be derived from the asteroid belt because - in that pre-chaos epoch - no specific dynamical processes had yet been identified that could bring asteroidal samples into Earth-crossing orbits. They criti- cized cosmochemist Ed Anders for not taking dynamics seriously when Anders argued that the meteorites must somehow come from the asteroid belt even if the dynamicists had not yet figured out how.

In both cases, the respective specialists insisted that it wasn't "scientific" to rely on unknown processes until the specific processes were proven to operate. Even as recently as last year I heard loud objections to the term "space weathering" because of the speaker's belief that we do not yet know for sure, from first principles, exactly how space weathering works. Yet, in both cases, history has demonstrated that the non-specialists who basically argued that anything was possible in the spheres of other fields so long as it wasn't specifically disproven turned out to be right. I would not extrapolate these cases to every scientific dispute and suggest that hand-waving generalists or non-specialists who ignore conservative specialists outside their fields of expertise will always prove to be right. But I think there are lessons to be learned from these case studies in asteroid science. Sometimes intuitively plausible hypotheses may prove to be true - provided they don't violate fundamental physical laws - even if we haven't yet figured out specifically how they could be true. We still operate in considerable ignorance and have much to learn, as I think Beth Clark's following talk will exemplify. I expect that studies of asteroid space weathering will still hold some surprises for us. 
THE NATURE OF SPACE WEATHERING EFFECTS ON ASTEROID 433 EROS. Beth Ellen Clark, Physics Department, Ithaca College, Ithaca NY 14850, USA, i bclark@ithaca.edu), Paul Lucey, University of Hawaii, HIGP, Honolulu HI 96822, USA, Paul Helfenstein, Astronomy Department, Comell University, Ithaca NY 14853, USA.

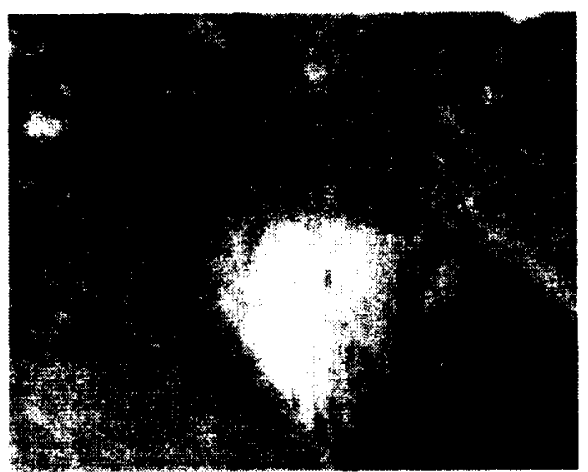

Figure 1: Near-Earth Asteroid Rendezvous (NEAR) observations of 433 Eros have revealed evidence for space weathering on an asteroid surface. This oblique view across the top of Psyche crater on Eros shows the steep northem slopes. Steep slopes allow gravitational processes to compete successfully with surface darkening processes.

Combined Multi-Spectral Imager (MSI) $(0.95 \mu \mathrm{m})$ and Near-Infrared Spectrometer (NIS) (0.8 $02.4 \mu \mathrm{m})$ observations of Psyche crater on 433 Eros are analysed to investigate the nature of space weathering effects on Eros. At $5.3 \mathrm{~km}$ in diameter, Psyche is one of the largest craters in Eros which exhibit distinctive brightness patterns consistent with downslope motion of dark regolith material overlying a substrate of brighter material (Figure 1). At spatial scales of $620 \mathrm{~m} / \mathrm{spectrum}$, Psy. che crater wall materials exhibit albedo contrasts of $32-40 \%$ at $0.946 \mu \mathrm{m}$. Associated spectral variations occur at a much lower level of $48 \%( \pm 2-4 \%)$.

In seeking to constrain the effects of space weathering processes on Eros, we have investigated several possible causes for the observed albedo and spectral trends: grain size differences, olivine, pyroxene, and troilite variations, and lunar-like optical surface maturation.

Spectroscopic data used for this analysis have been photometrically corrected [1]. Photometric correction procedures correct each spectrum for variations due to illumination and viewing geometry. The spectra we used were constrained to phase angles less than $100^{\circ}$, incidence and emission angles less than $75^{\circ}$, and spatial resolution $400-800 \mathrm{~m} / \mathrm{spectrum}$.

We use mineral mixture models to simulate the observed spectra. Next, we modify our mineral mixtures to simulate the spectral consequences of variations in grain size, composition, and optical maturity. We constrain our model mixture variations to be consistent with the observed albedo contrasts of $32-40 \%$. Finally, we compare model predicted spectral variation trends with the measured varialions.

We use an intimate mixing model based on the work of
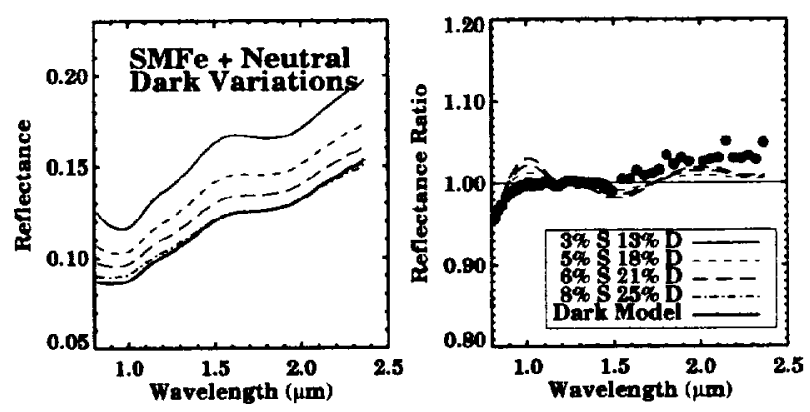

Figure 2: (left) The nominal mixture model spectrum of bright materials (top solid line spectrum) in Psyche is compared with mixture models made by increasing the proportions of both the neutral dark and the SMFe endmembers. (right) Each comparison spectrum is ratioed to the nominal spectrum and normalized to 1.0 at $1.3 \mu \mathrm{m}$. Also plotted are the observed dark/bright material spectral ratios (solid dots) to aid in comparison. This figure shows that the albedo contrasts and overall amplitude of spectral variation trends observed in Psyche crater can be simulated with a mixture model incorporating enhanced neutral dark and SMFe endmember components.

Hapke to quantitatively explore the effects of input parameters. Hapke [2] presents the methods necessary to compute the optical effects of submicroscopic iron which serves an important role in lunar space weathering optical effects. Our model allows the computation of the reflectance spectrum of a meteorite-like assemblage consisting of intimately mixed olivine, ortho- and clino-pyroxene, plagioclase feldspar, troilite, Fe-Ni metal, and submicroscopic Fe metal. We can compute the spectrum of an arbitrary mixture of these components at any grain size.

The model begins with a detinition of the relative abundances of the components and the mineral chemistries of the components For the transparent minerals (olivine, pyroxene, plagioclase and glass) we compute the optical constants from defined chemistries. Chemistry-dependent optical constant coefficients for olivine and pyroxene are from Lucey [3]. The chemistries of olivine and orthopyroxene were linked to conform to the relationship between olivine and pyroxene $\mathrm{Mg}$ number in ordinary chondrites from Keil and Fredrikkson [4]. Optical constants for glass as a function of iron content were computed by Lucey [3]. We used the Hapke-based methods presented in Lucey [3] to compute the iron dependent optical constants of plagioclase from reflectance spectra of plagioclase in the USGS Denver spectrum library.

Next, using the methods outlined in Hapke $[5,6,7,8]$ modified to include the effects of submicroscopic iron presented by Hapke [2] we compute the single scattering albedos of the 


\section{SPACE WEATHERING ON EROS: Beth Ellen Clark et al.}

transparent components from the optical constants, grain sizes and the defined abundance of submicroscopic iron, assuming a coating of metal for the minerals, and a uniform distribution of metal throughout the grain for glass. We note that lunar and asteroidal submicroscopic iron deposition may be quantitatively different because the asteroidal submicroscopic iron may also contain nickel. Our models assume that the submicroscopic coatings are pure iron, which appears to be a good assumption on the Moon Hapke [2]. The single scattering albedos are combined using equation (17) of Hapke [5]. The single particle phase functions are combined using the methods of Helfenstein et al. $[9,10]$. The mixture single scattering albedo is converted to reflectance using equation (37) of Hapke [2].

We find that the albedo contrasts in Psyche crater are not consistent with a cause due solely to variations in grain size, olivine, pyroxene or lunar-like optical maturation. A grain size change sufficient to explain the observed albedo contrasts would result in strong color variations that are not observed. Olivine and pyroxene variations would produce strong bandcorrelated variations that are not observed. A simple lunar-like optical maturation effect would produce strong reddening that is not observed. The contrasts and associated spectral variation trends are most consistent with a combination of enhanced troilite (a dark spectrally neutral component simulating optical effects of shock) and lunar-like optical maturation (Figure 2).

These results suggest that space weathering processes may affect the spectral properties of Eros materials, causing surface exposures to differ optically from subsurface bedrock. However, there are significant spectral differences between Eros' proposed analog meteorites (ordinary chondrites and/or primitive achondrites), and Eros' freshest exposures of subsurface bright materials.

Perhaps most surprisingly, after accounting for all differences in the measurement units of our reflectance comparisons, we have found that the bright materials on Eros have reflectance values at $0.946 \mu \mathrm{m}$ consistent with meteorites, but spectral continua that are much redder than meteorites from 1.5 to $2.4 \mu \mathrm{m}$. Most importantly, we calculate that average Eros surface materials are $30-40 \%$ darker than meteorites.

In summary, we find

(a) The background surface of Eros in and around Psyche crater is $32-40 \%$ darker than the bright materials exposed on steep slopes in the crater walls at spatial scales of approximately $620 \mathrm{~m} / \mathrm{spectrum}$.

(b) The dark material is $2-4 \%$ redder than the bright material at $1.5-2.4 \mu \mathrm{m}$, and has a $1-$ micron band which is $2-4 \%$ broader at $0.8-1.0 \mu \mathrm{m}$. The total spectral variation for the highest albedo contrast area found on Eros is $\mathbf{4 - 8 \%}$ peak-to-peak from the normalized mean at $1.3 \mu \mathrm{m}$.

(c) The albedo and contrasts in Psyche crater are not consistent with a cause due solely to either grain size or compositional variations.

(d) The contrasts and associated spectral variation trends observed in Psyche are consistent with a lunar-like optical maturation which combines accumulation of submicroscopic iron with an enhancement of the dark spectrally neutral component of Eros' surface materials (presumably shocked silicates).

(e) Bright materials in Psyche appear optically less mature than dark materials, but do not spectrally match meteorites The bright materials are approximately the same brightness as meteorites, however their spectra are $33 \%$ redder from 1.5 to $2.4 \mu \mathrm{m}$.

(f) We calculate that average Eros surface materials are 30 $40 \%$ darker than meteorites. We also find that when differences in reflectance measurement units and photometric geometry are accounted for, most meteorites are 2-3 times brighter than asteroids.

(g) If Eros is compositionally similar to ordinary chondrites, and if the darker materials on Eros are due to space weathering (optical maturation of a fresher brighter substrate), then the weathering process must be a strange two-step mechanism whereby materials are reddened before darkened (bright materials are redder than meteorites), and darkened more than reddened (albedo contrasts are stronger than color contrasts).

(h) At the scale of our observations Psyche crater did not excavate materials which are spectrally similar to meteorites. Nor does Psyche have any bright crater ejecta deposits, indicating that since the formation of the crater the surface of Eros has been weathered.

(i) These findings suggest that Eros's surface may be completely weathered due to its exposure to the space environment. Our results suggest that space weathering processes affect Eros' spectral properties, but do not fully explain the differences between Eros and its proposed analog meteorites, the ordinary chondrites and/or the primitive achondrites.

(j) Differences in optical maturation trends between Eros and other S-type asteroids studied by spacecraft may indicate different rates of the competing processes of surface maturation and impact cratering between objects in Near-Earth orbits and objects in the main asteroid belt.

\section{References}

[1] Clark, B.E. et al. (2002) NEAR Infraned Spectrometer Photometry of Asteroid 433 Eros. Icarus 155, 189-204. [2] Hapke, B. (2001) Space Weathering from Mercury to the Asteroid Belt. J. Geophys. Res. 106, 10,039-10,073. [3] Lucey, P.G. (1998) Model near-infrared optical constants of olivine and pyroxene as a function of iron content. J. Geophys. Res. 103, E1, 1703-1713. [4] Keil, K., and K. Fredriksson (1964) The Iron, Magnesium, and Calcium Distribution in Coexisting olivines and hombic pyroxenes of chondrites. J. Geophys. Res. 69, 3487-3515. [5] Hapke, B.W. (1981) Bidirectional Reflectance Spectroscopy I. Theory. J. Geophys. Res. 86, 3039-3054. [6] Hapke, B.W. (1984) Bidirectional Reflectance Spectroscopy III. Correction for Macroscopic Roughness. Icarus 59, 41-59. [7] Hapke, B.W. (1986) Bidirectional Reflectance Spectroscopy IV. The Extinction Coefficient and the Opposition Effect. Icarus 67, 264-280. [8] Hapke, B. (1993) Theory of Reflectance and Emittance Spectroscopy. pp.1-455 Cambridge University Press, New York. [9] Helfenstein, P., et al. (1994) Galileo Photometry of Asteroid 951 Gaspra. Icarus 107, 37-60. [10] Helfenstein, P., et al. (1996) Galileo Photometry of Asteroid 243 Ida. Icarus 120, 48-65. 
ANTS: APPLYING A NEW PARADIGM FOR LUNAR AND PLANETARY

EXPLORATION. P. E. Clark ${ }^{1}$ S. A. Curtis ${ }^{2}$, and M. L. Rilee ${ }^{1},{ }^{1}$ L3 Com Analytics Corporation, 2600 Park Towers Drive, Ste 800, Vienna, VA 22180 USA, Pamela.Clark@gsfc.nasa.gov, ${ }^{2}$ NASA/GSFC, Greenbelt Ruad. Greenbelt, MD 20771 USA, u5sac@lepvax.gsfc.nasa.gov.

Introduction: ANTS (Autonomous NanoTechnology Swarm), a mission architecture consisting of a large (1000 mernber) swarm of picoclass $(1 \mathrm{~kg})$ totally autonomous spacecraft with both adaptable and evolvable heuristic systems, is being developed as a NASA advanced mission concept $[1,2]$, and is here examined as a paradigm for lunar surface exploration.

As the capacity and complexity of hardware and software, demands for bandwidth, and the sophistication of goals for lunar and planetary exploration have increased, greater cost constraints have led to fewer resources and thus, the need to operate spacecraft with less frequent human contact At present, autonomous operation of spacecraft systems allows great capability of spacecraft to 'safe' themselves and survive when conditions threaten spacecraft safety. To further develop spacecraft capability, NASA is at the forefront of development of new mission architectures which involve the use of Intelligent Software Agents (ISAs), performing experiments in space and on the ground to advance deliberative and collaborative autonomous control techniques. Selected missions in current planning stages require small groups of spacecraft weighing tens, instead of hundreds, of kilograms to cooperate at a tactical level to select and schedule measurements to be made by appropriate instruments onboard. Such missions will be characterizing rapidly unfolding real-time events on a routine basis. The next level of development, which we are considering here, is in the use of autonomous systems at the strategic level, to explore the remote terranes, potentially involving large surveys or detailed reconnaissance.
One Previous Application: The use of this approach has already been discussed $[1,2]$ for mainbelt asteroids, a population consisting of more than a million small bodies. Although a large fraction of solar system objects are asteroids, relatively little data is available for them because the vast majority of them are too small to be observed for more than single point measurements except in close proximity. Determination of the systematic distribution of physical and compositional properties within the asteroid population is crucial in the understanding of the solar system formation. In addition, there has been interest in asteroids as sources of exploitable resources. Far more reconnaissance is required before either goal is achieved. A traditional mission approach (to explore larger asteroids sequentially) is not adequate for studying the asteroid population systematically. Our approach involves the use not of a smart spacecraft with 'drones', but of a totally autonomous truly distributed intelligent network of sensors. Each sensor will have specialized instrument capability (e.g., advanced computing, imaging, spectrometry, etc.) and heuristic systems that are both adaptable and evolvable, to simultaneously evaluate a broad cross-section of the entire population. Spacecraft use solar sails, which require no expendables, as the propulsion systems needed to fly from the Earth directly to asteroids 1 kilometer or greater in diameter. Clusters of spacecraft operate simultaneously within a broadly defined framework of goals to select targets from among available candidates and to develop scenarios for studying targets, and selected members of the swarm return to Earth with data and are replaced as needed. 
Application to Lunar Exploration: The ANTS concept has broad application to planetary targets in the inner solar system where detailed reconnaissance is a next logical step in exploration, but the cost and capabilities of lander or rover technology are still limiting factors. The Moon would be an ideal candidate for such a totally autonomous investigative approach using stationkeeping or low- periapsis solar sailors, each with a specialized instrument or capability and placed optimally for its viewing requirements. The many lunar remote sensing investigations, limited in coverage or instrumentation or both, which have already have been done provide an essential context for the step proposed here. A comprehensive simultaneous multi-sensor survey for evidence of the deposits of ice or volcanic material inferred to be buried or just beneath the surface, could provide essential clues on the formation and history of, for example, polar regions, antipodal magnetic swirls, KREEP, the farside basins, to name a few candidate sets of features. Or, clusters of spacecraft could be used to do a systematic highest possible resolution survey of the entire lunar surface. Sensors to be considered should include visible and near infrared red imager/spectrometers, X-ray,
Gamma-ray and neutron spectrometers, altimeter/ranger/sounders, magnetometers. Spacecraft could work in same sensor vertical or planar arrays for $2 \mathrm{D}$ or $3 \mathrm{D}$ imaging or field determination, for example, using magnetometers to investigate antipodal magnetic swirls. Alternatively, multisensor clusters would allow optimal data fusion [3] when prospecting for a particular material, such as neutron, gamma-ray, and $\mathrm{X}$-ray spectrometers, along with radio sounders, all with relevant compositional information to allow interpretation of measurements in terms of the presence and distribution of ice. The lunar environment is more familiar (to humans), and Earth/Moon communication is near real time. As a result, the Moon presents a lower risk than other targets and would be an ideal place for ANTS to train and for the totally autonomous distributed intelligence concept, and human/machine interfaces generally, to undergo development and testing without the expense of sending humans into space.

References: References: [1] Curtis et al, (2000) IAU Proceedings, IAF-00-Q.5.08. [2] Rilee, Clark, and Curtis, (2002) LAU Proceedings, in preparation. [3] Clark and McFadden (2000) JGR, 105, 4291. 


\section{PLANETARY REgOLITH SURFACE ANALOGS AND MESOSCALE TOPOGRAPY: OPTIMIZED DETERMINATION OF HAPKE PARAMETERS USING MULTI-ANGULAR SPECTRO-IMAGING LABORATORY DATA.}

A. Cord', P. Pinet', Y. Daydou1, D Stankevich ${ }^{2}$ and Yu. Shkuratov'. 'Observatoire Midi-Pyrénées (14 Av. E. Belin, 31400 Toulouse, France. Aurelien.C 'ord@enes.fr). ${ }^{2}$ Kharkov Astronomical Observatory (35 Sumskaya St., Kharkov 61022, Ukraine.).

Introduction: Reflectance spectroscopy is now a tool widely used for remote sensing applications, both in planetary and terrestrial observation. Among the large number of possibilities offered by this technique, the investigation of the surface mineralogy of a target is often explored. However, with the considerable increase of the spatial resolution of uptical experiments on and near the planetary surfaces, it becomes more and more important to understand the physics that control the light interaction process with soils, in order to better interpret the physical (nature, texture, surface roughness, maturity, degree of cristallinity) and mineralogical properties of the surface at subpixel scale.

Over the years, a number of semi-empirical models have been developed for analyzing the bidirectional reflectance of particulate surfaces on the basis of the scattering and absorption propertics of minerals and rocks. In particular, Hapke [1] developed an approximate analytical solution, which provides a description of the photometric behavior in terms of physically meaningful parameters.

This work uses a method, founded on a genetic algorithm, for a determination of the global set of parameters involved in Hapke's model for planetary surface analogs when dealing with a set of angular conditions representative of the usual range of observation in planetary exploration for spaceborne optical instruments.

Experimental measurements: Based on experimental measurements produced by a new laboratory widefield multispectral imaging facility [2], the method is applied to three soils: two of them are light materials (single scattering albedo about 0.7 ) and one is dark material (single scattering albedo about 0.3). Those soils are sorted to four grairi sizes, and are representative of the spectral variability of observations realized on planetary surfaces.

The grain size sorting is the following: less than $75 \mu \mathrm{m}$ (labeled "Gl"), from 75 to $250 \mu \mathrm{mi}$ ("G2"), from 250 to $500 \mu \mathrm{m}$ ("G3") and from $500 \mu \mathrm{m}$ to $2 \mathrm{~mm}$ ("G4").

The domain of geometries of obselvation investigated is controlled by the observational situations the most frequently met in planetary exploration by spaceborne optical instruments (e.g. MOC/MGS, HRSC/MarsExpress, AMIE/Smart-1, LIC/Lunar-A, LISM/Selene). The incidence angle is selected between $0^{\circ}$ and $55^{\circ}$ and the emergence angle between $-70^{\circ}$ to $0^{\circ}$ (azimuth angle $0^{\circ}$ ) and $0^{\circ}$ to $70^{\circ}$ (azimuth angle $180^{\circ}$ ) with a minimum phase angle of $20^{\circ}$.

In this range, ten measurements are produced in order to span the photometric variability.

Results: The Hapke's formalism using the parameter values determined by the genetic algorithm well models the variations in bidirectional reflectance related to particle grain size distribution for the three materials. It establishes that all the steps in our methodology are sound, including experimental measurements, the derivation of photometric quantities by means of Hapke's model and the use of genetic algorithm for an optimized determination of the Hapke parameters [3].

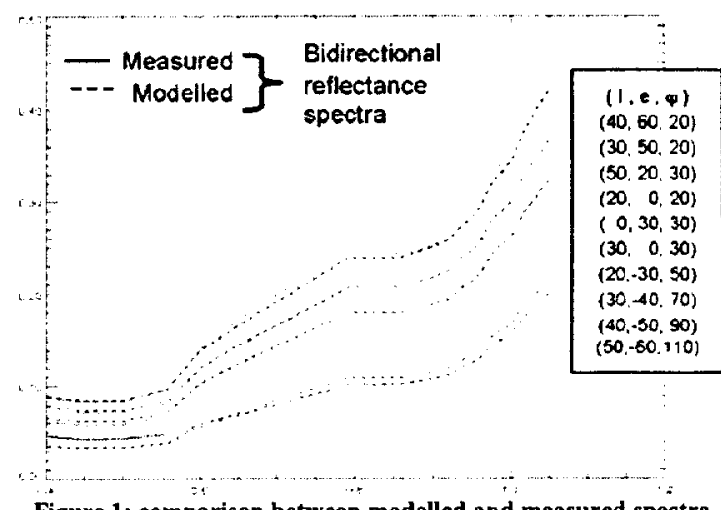

Figure 1: comparison between modelled and measured spectra for one of the twelve samples.

Furthermore a by-product of this application is the derivation of Hapke parameters for samples representative of materials found on planetary soils, which may be used in future reflectance spectroscopy data interpretations:

Phase function parameters (double Henyey-Greenstein function, [4]):

- b keeps similar values for all samples, between 0.4 and 0.5 .

- $\quad$ generally increases with grain size. 
OPTIMIZED DETERMINATION OF HAPKE PARAMETERS, A. Cord et al.

Those parameters are well constrained, showing that the angular domain available in this study (phase angle between $20^{\circ}$ and $120^{\circ}$ ) is large enough to determine this function.

Backscattering function parameters:

- $B_{0}$ values are very small, for our dark material (the backscattering effect is negligible) and for our light materials, $B_{0}$ values increase with grain sizes from 0 for $\mathrm{G} 1$ to 0.6 for $\mathrm{G} 4$.

This shows that materials with larger grains have a structure containing more tunnels, creating a stronger backscattering effect. This result is due to our target experimental design where the surfaces of samples with small grain sizes have a smoother aspect than those with larger grain sizes, implying that, when grains become smaller, the textural roughness of the abserved surface decreases.

- $h$ value ranges about 0.15 for the Palagonite sample and about 0.55 for the Tephra sample, when the backscattering effect is not negligible ( $B_{0}$ greater than 0.2).

Given the limited mesoscale topography range (see below), those values could not be interpreted as it is usually done, because there is an interaction with topographic shadowing function (see in this volume the companion abstract by Stankevich et al.).

Backscattering function is less constrained than phase function. In fact to better describe backscattering effect, smaller phase angle measurements are needed. Roughness function:

- $\theta$ values are highly correlated with grain sizes for our light material samples: $14^{\circ}$ for $\mathrm{Gl}, 20^{\circ}$ for $\mathrm{G} 2,23^{\circ}$ for $\mathrm{G} 3$ and $26^{\circ}$ for $\mathrm{G} 4$. Darker material is linked with smaller $\theta$ values.

In the case of real planetary observations, we may consider three levels of scale (cf. table I): first, the largest one is the topographic scale representing the surface topography variations greater than the pixel size, which ranges typically between 5 and $100 \mathrm{~m}$ scale for current and existing measurements (e.g. MOC/MGS, HRSC/Mars-Express). Then, the smallest scale describes the particulate medium and is controlled by the grain size distribution, which goes from a few tens of microns up to a few hundreds microns. Intermediate to these two scales, exists the mesoscale structure that is linked to the topographic textural distribution inside each pixel, and is represented in Hapke's theory by the $\theta$ parameter, referred to as the surface roughness.

In this study, the pixel size is about $3 \mathrm{~cm}$ and grain size range goes from a few tens of microns (for G1) to 2 $\mathrm{mm}$ (for $\mathrm{G} 4$ ). As a result, the mesoscale domain is reduced in scale (from $\mathrm{mm}$ to a $\mathrm{few} \mathrm{cm}$ ) in our investigation.

\begin{tabular}{|l|c|c|c|}
\hline & $\begin{array}{c}\text { Particulate } \\
\text { Medium } \\
\text { Grain Size }\end{array}$ & $\begin{array}{c}\text { Textural } \\
\text { Mesoscale } \\
\text { Topography }\end{array}$ & $\begin{array}{c}\text { Observation } \\
\text { Scale } \\
\text { Pixel Size }\end{array}$ \\
\hline $\begin{array}{l}\text { Planetary } \\
\text { Observations }\end{array}$ & $10-200 \mu \mathrm{m}$ & $1 \mathrm{~mm}-1 \mathrm{~m}$ & $5-100 \mathrm{~m}$ \\
\hline $\begin{array}{l}\text { Laboratory } \\
\text { Measurements }\end{array}$ & $10-200 \mu \mathrm{m}$ & $1 \mathrm{~mm}-1 \mathrm{~cm}$ & $3 \mathrm{~cm}$ \\
\hline \multicolumn{3}{|c|}{$\begin{array}{c}\text { Table I: Three considered levels of seale } \\
\text { for planetary observation and laboratory }\end{array}$}
\end{tabular}

The values found by the genetic algorithm are in the same range as those determined in McEwen study (1991) for planetary soils: it establishes that the algorithm gives plausible values despite the change in scale of observation implying a more reduced range for the mesoscale features (cf. table I). Indeed, our experiment shows that in case of large grain sizes in the millimeter range, the textural topography variation inside the pixels is dominated by the "rocky" aspect of the surface, this is not yet the case in planetary observation. However with the increase of the spatial resolution in orbital imaging or for in-situ measurements, this situation could arise.

Conclusion: Hapke's model implicitly considers that the scale of surface topography is much larger than the size of regolith particles handled as a particulate medium. However, in laboratory and in-situ planetary measurements one deals with surface topographies that have the characteristic scale comparable with the size of regolith particles, and often such mesoscale topographies are formed by rock fragments. This study shows that despite the change of scale, the Hapke's formalism may describe the photometric behavior of the surface, but the meaning of $\theta$ and $h$ parameters becomes more difficult to handle with the possibility of ambiguous physical interpretation. Theses results point at the need for an improved theoretical modeling for describing the real case of natural regolithic surfaces, for which the mesoscale organization of the textural relief influences the surface photometric behavior (see in this volume the companion abstract by Stankevich et al.)

Acknowledgement.

References: [1] Hapke B. (1993) Theory of reflectance and emittance spectroscopy. Cambridge Univ. Press. 450 p. [2] Pinet et al. (2001) LPSC XXXI, Abstract 1559. [3] Cord A. et al. (2002) lcarus (submitted). [4] Hartman, B., and D. Domingue (1998) Icarus 131, 421-448. 
TOWARD AN IMPROVED SINGLE-PARTICLE MODEL FOR LARGE IRREGULAR GRAINS. W. M. Grundy, Lowell Observatory, 1400 W. Mars Hill Rd., Flagstaff AZ 86001 (grundy@lowell.edu), B. Schmitt and S. Douté, Laboratoire de Planétologie de Grenoble, Bat. D de Physique B.P. 53, 38041 Grenoble Cedex 9, France.

To interpret remote spectral observations, scattering and absorption in a particulate surface are simulated via radiative transfer models. The standard model for this purpose among the planetary science community is the Hapke model (e.g., Hapke 1981, 1993, 2002). This model (like many others) uses two parameters to characterize the optical behavior of individual grains in a particulate surface, the single-scattering albedo $w$ and phase function $p(g)$. These terms describe, respectively, the quantity and the angular distribution of light scattered by an individual grain. Unfortunately, these parameters are strictly optical. They can be rather difficult to interpret in terms of more interesting particle properties such as grain sizes, shapes, and compositions, that a remote sensing experiment might seek to discover.

An equivalent slab approximation is 1ypically used to relate $w$ to the grain size and optical constants of the material. This approach can mimic the wavelength-dependent absorption behavior of irregular grains, as long as the imaginary index $k$ is much less than 1, the shape is equant, and the grain size $D$ is much larger than the wavelength $\lambda$. Unfortunately, the equivalent slab approach provides no information about $p(g)$, which also has a strong dependence on optical constants and particle form (e.g., Grundy et al. 2000). Instead, $p(g)$ is commonly approximated with simple analytical functions such as Legendre polynomials or Henyey-Greenstein functions (e.g., Hapke 1993), effectively discarding the compusitional and textural information carried by the wavelength-dependence of $p(g)$. Often $p(g)$ is assumed to be independent of wavelength (e.g., Cruikshank et al. 1998, Quirico et al. 1999, Doute et al. 1999), thereby forcing the single-scattering albedo to account for all observed spectral features. Such an assumption can lead to significant errors, especially in simulating layered media (e.g. Johnson \& Grundy 2001).

There are many ways of computing or empirically measuring $p(g)$ for an irregular grain having specified optical constants and form (e.g., microwave analogs, Gustafson 2000; discrete dipole approximations, Draine 2000; Monte Carlo ray-tracing, Macke 2000; etc.). Unfortunately, none of these techniques is fast enough for use in iterative radiative transfer models. Using equivalent spheres simulated via Mie theory offers the advantage of providing $w$ and $p(g)$ simultaneously, but such a model also has difficulties. It is still too computationally expensive, and its application to grains in contact with one another (as in a planetary surface) is somewhat inappropriate, since diffraction is included without considering the effect of neighboring grains.

A new, much less computationally expensive, approximate model is urgently needed for calculating $w$ and $p(g)$ from optical constants and particle forn within iterative remote sensing radiative transfer models. A new single particle model would be especially valuable in newer models which are more accurate in their accounting for the angular distribu- tion of multiply-scattered light (e.g., Douté \& Schmitt 1998 , Cheng \& Domingue 2000, Hapke 2002)

We will present a status report on our ongoing effort to remedy this situation by developing a new model of $w$ and $p(g)$ for large, irregular grains. Previous studies have established that $w$ and $p(g)$ exhibit consistent trends as functions of panicle characteristics (e.g., McGuire \& Hapke 1995, Hartman \& Domingue 1998). We are capitalizing on this regular behavior by using it to constrain simple models. We began by performing a Monte Carlo ray-tracing study of scattering by large, irregular particles (Grundy et al. 2000). Figure 1 shows results from this study, in which the Henyey-Greenstein parameters of $p(g)$ are seen to vary with absomtion $\alpha D$, refractive index $n$, and particle surface roughness. Our studies with the Monte Carlo model continue, with the results being used to constrain and test candidate models of $w$ and $p(g)$ as functions of grain size, form, and optical constants over a broad range of parameter space for large irregular particles.

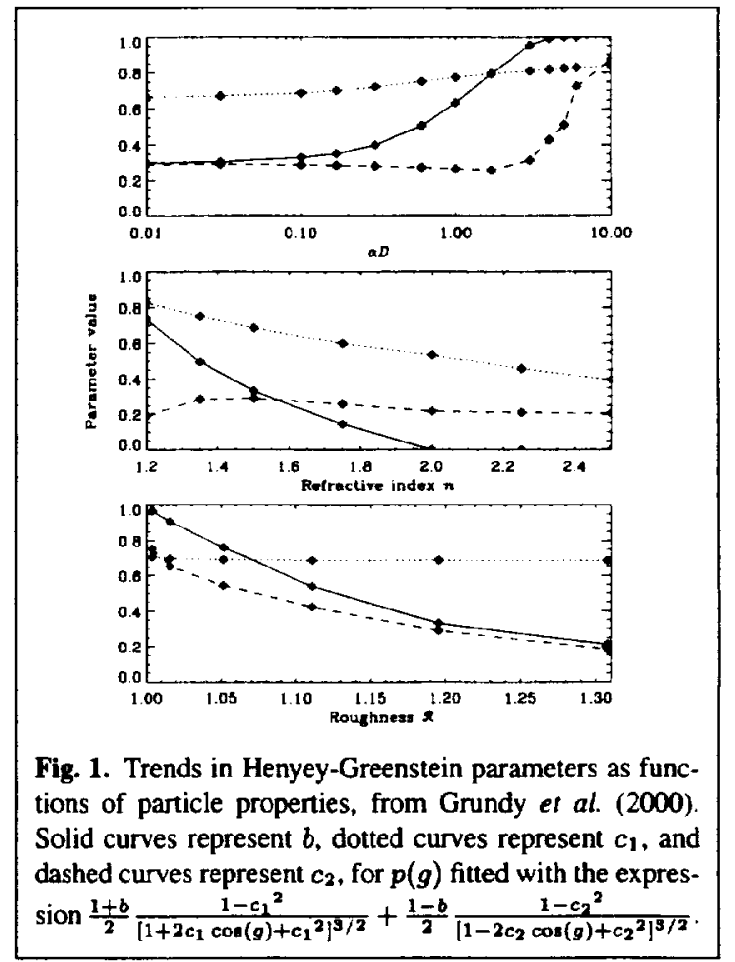

For each Monte Carlo run, we record distinct populations of rays separately: i.e., those reflected from the grain's exterior, those transmitted once through the grain, those transmitted with one internal reflection, those transmitted with two internal reflections, etc. In addition to the angular distribution for scattering from each sub-population, we record the mean in- 


\section{NEW IRREGULAR PARTICLE MODEL: Grundy, Schmitt, \& Douté}

ternal path lengths, polarizations, etc. Each of these scattered populations, as shown in Fig. 2, has a characteristic amplitude and angular distribution, which is directly dependent on particle properties such as optical constants, shape, and surface texture.

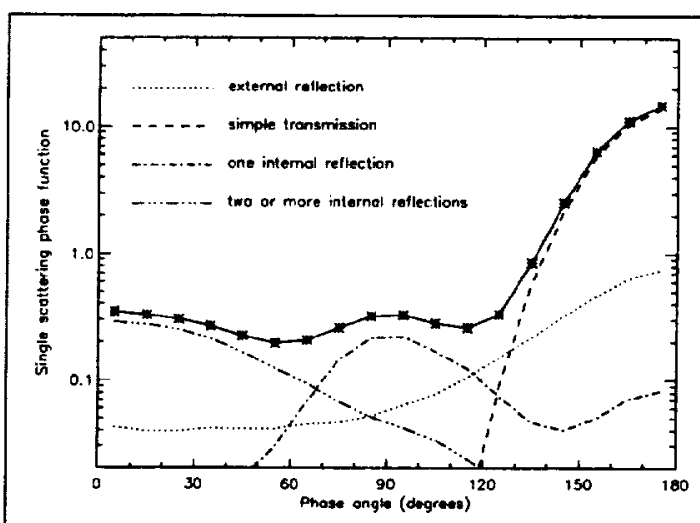

Fig. 2. Distinct populations of rays contributing to $p(g)$ for a single irregular particle with $n=1.3$ and $\alpha D=1$.

Our objective is to represent scattering by each of these separate populations using simple functional forms such as Henyey-Greenstein functions or Legendre polynomials. We are attempting to develop semi-analytical/semi-empirical expressions for the amplitudes and anguiar distributions of these distinct contributions to $p(g)$, which can then be summed to obtain computationally inexpensive synthetic $p(g)$ for arbitrary irregular grain parameters. From the estimated amplitude of each contribution, we can also compute $w$ from the mean interior path lengths for the different ray classes, combined with the absorption coefficients and the Beer-Lambert law.

It remains to be determined how many separate ray classes must be considered to achieve acceptable levels of accuracy. For weakly-absorbing particles (especially those with high refractive indices), the contribution from rays having been inter- nally reflected numerous times can be significant. Fortunately, this highly-scattered component of $p(g)$ tends to have a relatively uniform angular distribution. We are investigating the possibility of merging all the highly-scattered components into a single isotropic or back-scattering term, having an amplitude estimated from the interior scattering efficiency $S_{i}$ and particle opacity $\alpha D$ in a manner analogous to the equivalent slab approximation.

ACKNOWLEDGEMENTS: Support for this project is being provided by NASA Planetary Geology and Geophysics grant NAG5-10159 and by the Programme National de Planétologie de l'INSU.

\section{References}

Cheng \& Domingue 2000, J. Geophys. Res. 105, 9477-9482.

Cruikshank et al. 1998, Icarus 135, 389-407.

Douté \& Schmitt 1998, J. Geophys. Res. 103, 31367-31389.

Douté et al. 1999, Icarus 142, 421-444.

Draine 2000, in Light Scattering by Nonspherical Particles, Academic Press, San Diego.

Grundy et al. 2000, J. Geophys. Res. 105, 29291-29314.

Gustafson 2000, in Light Scattering by Nonspherical Parricles, Academic Press, San Diego.

Hapke 1981, J. Geophys. Res. 86, 3039-3054.

Hapke 1993, Combined Theory of Reflectance and Emittance Spectroscopy, Cambridge University Press, New York.

Hapke 2002, Icarus 157, 523-534.

Hartman \& Domingue 1998, Icarus 131, 421-448.

Johnson \& Grundy 2000, Geophys. Res. Lett. 28, 2101-2104.

Macke 2000, in Light Scattering by Nonspherical Paricles, Academic Press, San Diego.

McGuire \& Hapke 1995, Icarus 113, 134-155.

Quirico et al. 1999, Icarus 139, 159-178. 
BACKSCATTER EFFECTS OF PARTICLES WITH PERFECT AND IRREGULAR SHAPES: GEOMETRIC OPTICS APPROACH. Ye. Grynko ${ }^{1.2}$ and Yu. Shkuratov ${ }^{1},{ }^{1}$ Kharkov astronomical observatory, 35 Sumskaya St., Kharkov 61022, Ukraine, ${ }^{2}$ Max-Planck-Institut für Aeronomie, Max-Planck-Str., 2, KatlenburgLindau D-37191, Germany, e-mail: ধrynko@linmpi.mpg.de.

Introduction: To interpret the: opposition phenomena observed for regolith surfaces optical properties of individual particles should be studied. Even under neglecting diffraction, the problem of light scatter by particles of irregular shape is rather complex. Using the computer ray-tracing metlod, see, e.g, [1.2], we investigate here brightmess and polarization backscatter effects of particles with different shapes in the geometric optics approximation.

Model description: We use a computer Monte Carlo ray tracing approach [2] that allows us calculations of the particle scattering indicatrix with accounting for polarization. A particle is gelserated in the computer memory. It is presented by a succession of triangular flat facets, for which the laws of reflection and refraction are applicable. Thus one can put a normal to each facet of any particle and calculate all necessary local angles. Then, the particle is "illuminated" with great enough number of rays. These rays are multiply reflected and refracted on their way. Besides, they can be partly absorbed within the particle, if it is semitransparent. The interaction with the particle surface is described by means of the Fresnel and Snell laws.

In this work we concentrate on the comparison of optics of particles of perfect shapes widely used in various models of scattering to data for their nonperfect analogs and randomly shaped particles. Several classes of irregular particles are exarmined: (1) faceted spheres that have a different number of flat facets, $N$, which varies in our studies from 100 to 30000 ; (2) cubes of "spoiled" forms, when the length of cube sides slightly varies; (3) randomly shaped particles generated by an auxiliary random gaussian field. The latter is different from so called gaussian particles described in [1], where they are assumed to be stochastically deformed spheres, the radius of which follows to the lognormal statistic law. In order to generate the particles of this class we use an auxiliary 3-D random gaussian field (RGF). After generating such a field, its dissection at a certain level is made that gives a 3-D medium consisting of particles with randon shapes and sizes that could be called RGF particles [3].

We have thoroughly tested the model. In particular, the algorithm was verified on the reciprocity principle expressed in the vector form. The quality of averaging-out over particle orientations has been checked by the criterion of smallness of the elements $F_{13}, F_{14}$, $F_{23}$ и $F_{24}$ and by the condition of symmetry $F_{12}=F_{21}$ and anti-symmetry $F_{34}=-F_{43}$. Although here we pre- sent only intensity and polarization curves, our model allows calculation of all non-zero elements of scattering matrix [3].

Results: The element $F_{11}$ and ratio $-F_{12} / F_{11}$ of scattering matrix vs. phase angle $\alpha$ for faceted spheres having different numbers of facets and RGF particles are shown in Fig. 1 at $n=1.5$ (refractive index) and $k=0$ (absorption coefficient). Results were averaged over orientations and shapes (in case of RGF particles). A spherical on average particle formed with facets, a number of which is more than approximately 10000 , can be considered as a rather perfect sphere. When $N$ is lesser, great changes are observed. Thus, for $F_{11}$ at

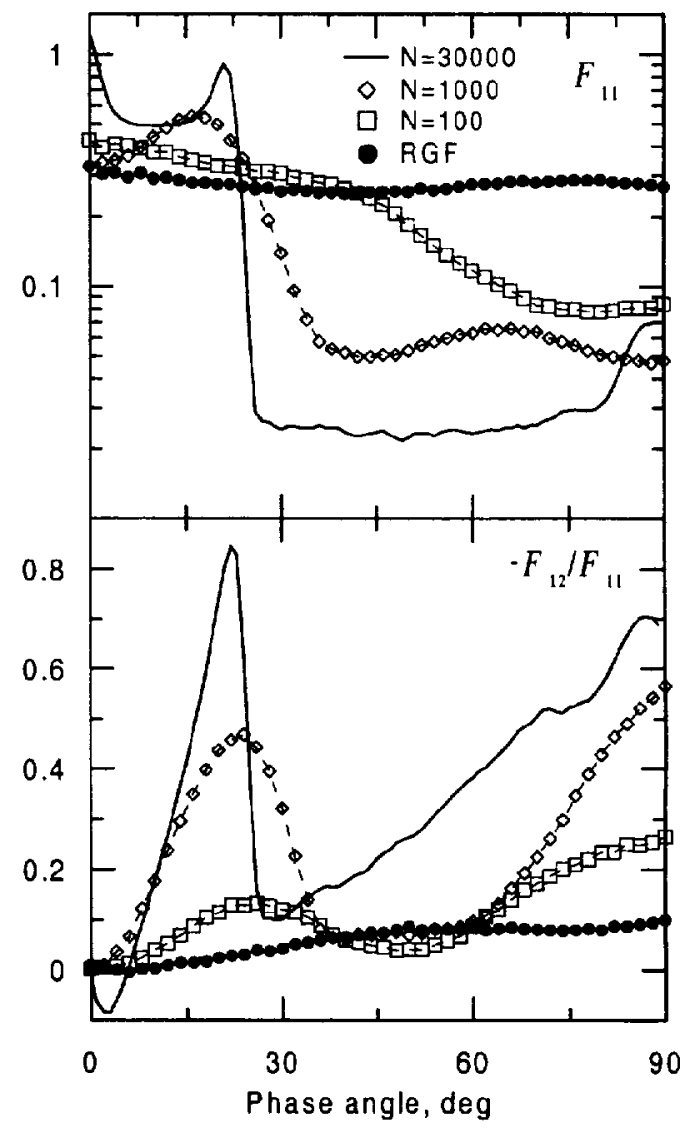

Fig. 1. Phase functions of brightness and linear polarization degree for spherical particles approximated by different number of facets $N$ and for RGF particles at $n=1.5$ and $k=0$. 
$N=1000$ the glory surge disappears and, moreover, instead of the surge an opposition "anti-spike" is developed. At $N=100$, neither the glory nor the first rainbow are observed. Weakening of the features with the decrease of $N$ is clearly seen for the ratio $-F_{12} / F_{11}$. This ratio corresponds to the definition of linear polarization degree of scattered radiation, when particles are illuminated with unpolarized light. So, as one can see in Fig. 1, the small negative polarization branch, which is observed for the perfect spheres near the backscatter direction disappears for roughly faceted particles. The surge of positive polarization corresponding to the first rainbow goes down quickly with decreasing $N$.

The phase function for RGF particles is almost constant in the concerned range of phase angles. It does not show any presence of the rainbow or opposition spike. Light scattered by this kind of particles is positively polarized in backward direction and the polarization degree is much less than that for spheres.

In Fig. 2 the results for perfect and randomly deformed cubes are represented. To obtain particles with such shapes, we used perfect cubes deforming them in the following way. Each vertex of a cube is randomly moved in a random direction. The shift has the zero average and the certain amplitude $\delta$ that is given in the length of the cube edge. We varied $\delta$ from 0 (perfect cube) to 0.2 (very irregular particle). As in the case of other particles the irregular cubes are approximated with triangle facets. The ideal cube reveals the brightness spike as well as strong negative polarization branch at small $\alpha$. These results are in a good agreement with [4]. The backscatter spike is caused by the component of scattered radiation corresponding to double internal reflection and is quickly degraded with deviation of particles from cubical shape. This component also contributes very strongly to the negative polarization that is rapidly relaxed with growing $\delta$.

Conclusions: Scattering properties of faceted spheres with number of the facets less than 10000 distinct significantly from those of the perfect spherical particles. The phase angle curves of the element $F_{11}$ and ratio $-F_{12} / F_{11}$ (and other scattering matrix elements too) of roughly faceted spherical particles, e.g., with $N=100$, diverge very much from those of perfect sphere. The irregular particles show smoother curves. Even small deviations from the perfect shape kill the opposition spikes and negative polarization branch. The ideal cube exhibits the brightness spike as well as strong negative polarization at small $\alpha$. When the cubes are deformed the spike are reduced and become wider; the negative polarization branch vanishes.

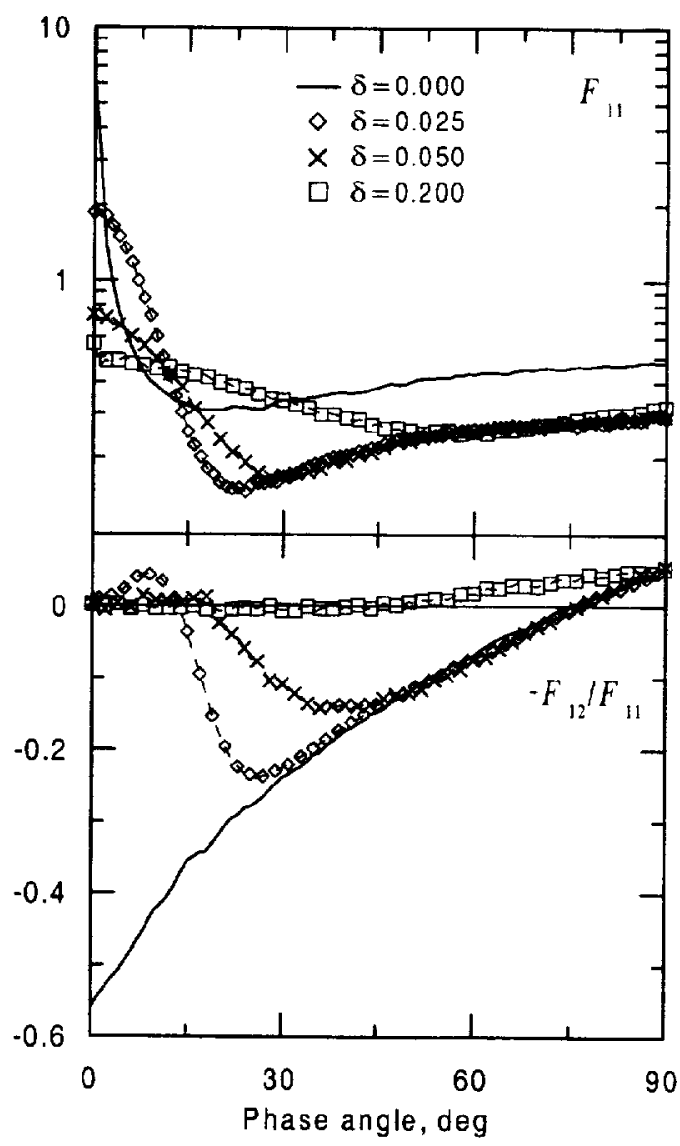

Fig. 2. Phase functions of brightness and linear polarization degree for particles with shapes randomly deviating from cube at $n=1.5$ and $k=0$.

Our modeling shows that there is qualitative similarity of the phase angle curves of intensity $F_{11}$ and ratios $-F_{12} / F_{11}$ (as well as of $F_{34} / F_{11}, F_{22} / F_{11}, F_{33} / F_{11}$, $F_{44} / F_{11}$ ) for the roughly faceted spheres (ellipsoids), RGF particles, and irregular cubes with $\delta=0.2$. Thus strongly irregular particles of all studied classes reveal much more resemblance in scattering properties, than their perfect representatives.

References: [1] Peltoniemi J. et al. (1989) Appl Opt., 28, 4088-4095. [2] Stankevich D. et al. JQSRT, in press. [3] Grynko Ye. and Shkuratov Yu. JQSRT, submitted. [4] Muinonen K. (1989) Appl. Opt., 28, 3051-3060. 
MARS RADAR OBSERVATIONS WTTH THE GOLDSTONE SOLAR SYSTEM RADAR. A. F. C. Haldemann', R. F. Jurgens', K. W. Larsen ${ }^{2}$, R. E. Arvidson ${ }^{2}$, and M. A. Slade', ' Jet Propulsion Laboratory, California Institute of Technology, 4800 Oak Grove Dr., Pasadena, CA 91109-8099, USA. ${ }^{2}$ Dept. of Earth and Planetary Sciences, Washington University, 1 Brookings Drive, St. Louis, MO 63130. Email: albert@shannon.jpl.nasa.gov.

Introduction: The Goldstone Solar System Radar (GSSR) has successfully collected rildar echo data from Mars over the past 30 years. As such, the GSSR has played a role as a specific mission element within Mars exploration. The older data provided local elevation information for Mars, along with radar scattering information with global resolution (e.g. $[1,2]$ ). Since the upgrade to the 70-m Deep Space Network (DSN) antenna at Goldstone completed in 1986, Mars data has been collected during all but the $1997 \mathrm{Ma}$ s opposition. Radar data, and non-imaging delay-Doppler data in particular, requires significant data processing to extract elevation, reflectivity and roughness of the rellecting surface [3]. The spatial resolution of these experiments is typically some $20 \mathrm{~km}$ in longitude by some $15 \mathrm{okm}$ in latitude. The interpretation of these parameters while limited by the complexities of electromagnetic scsitering, do provide information directly relevant to geophysical and geomorphic analyses of Mars.

Landing Site Assessment with Rudar Data. The usefulness of radar data for Mars exploration has been demonstrated in the past. Radar data were critical in assessing the Viking Lander 1 site $[4,5]$ as well as, more recently, the Pathfinder landing site: $[6,7]$. In general, radar data have not been available to the Mars exploration community at large. A project funded initially by the Mars Exploration Directorate Scierce Office at the Jet Propulsion Laboratory (JPL), and liter funded by NASA's Mars Data Analysis Program has reprocessed to a common format a decade's worth if raw GSSR Mars delay-Doppler data in aid of landing site characterization for the Mars Program. These data will soon be submitted to the Planetary Data System (PDS). The radar data used were obtained between 1988 and 1905 by the GSSR, and comprise some 63 delay-Doppler radar tracks. Of these, 15 have yet to be recovered from old 9-track tapes, and some of the data may be permanently lost. The available data used for Mars Exploration Rover landing site analysis for example, are listed in Table 1.

Enhanced Radar Observations: Two modern radar techniques offer the opportunity to improve the spatial resolution and the global coverage of available radar
data:random-long-codedelay-Doppler observations, and interferometric delay-Doppler observations.

Random-long-codedelay-Dopplerradar. In standard delay-Dopplerobservations, Mars isoverspread [8] This can be overcome by using a pseudo-random, nonrepeating code that is much longer than the observing run. This technique was successfully applied to Mars by Harmon et al. [9] to image the Martian surface at $39 \mathrm{~km}$ resolution with the Arecibo Observatory at $12.6 \mathrm{~cm}$ wavelengths. The GSSR used the technique at $3.5 \mathrm{~cm}$ during the 1999 opposition [10]. Dual-polarization coded long pulse data were collected. The 1999 opposition provided the opportunity to observe Mars at high northern sub-radar latitudes, providing a good view of the north polar region. The campaign resulted in 14 days of data over the course of a month. The GSSR $25 \mathrm{kHz}$ baud rate used in 1999 had the potential to produce 12 $\mathrm{km}$ resolution cells in the polar regions. For signal-tonoise reasons, the actual cell size was $48 \mathrm{~km}$. The random-long-code technique is particularly useful for obtaining radar information away from the sub-Earth radartrack at moderate to high latitude. The north-south ambiguity inherent in delay-Doppler is still manifest, and so the technique really lends itself to observations of bright features on the surface.

Interferometric delay-Doppler radar. Observing the radarecho with more than one receiver provides a means to remove the north-south delay-Doppler ambiguity. Ten of the 63 radar tracks in the recently re-assembled radar dataset are interferometric radar tracks. The interferometric information has never been processed, because the signal to noise is insufficient to constrain both the phases and the radar scattering parameters. The new topographic data from the Mars Orbiter Laser Altimeter (MOLA) on the Mars Global Surveyor (MGS) spacecraft offer the best means to analyze these unused data to make radar maps that extend the radarproperties coverage some 3 to 4 degrees beyond the sub-earth radar track. This will be a significant expansion of the dataset, and is all the more warranted as the radar spatial resolution improves away from the sub-Earth track (smaller range ring-Doppler strip intersections away from the subEarth track). At the outer edges the radar resolution cell 
GSSR Mars Data: A. F. Haldemann et al.

is of the same order of size as the landing site ellipses for future mission (approximately $20 \mathrm{~km}$ diameter). Progress on this new technique is reported by Larsen et al. at this meeting, in particular as regards data acquired during the 2001 Mars opposition.

References: [1] Goldspiel J. M. et al. (1993) Jcarus, 106, 346-364. [2] Moore H. J. and Thompson T. W. (1991) $L P S$ XX7, 812-815. [3] Hagfors T., JGR, 102, 3779-3784. [4] Masursky H. and Crabill N. L. (1976) Science, 193, 809-812. [5] Tyler G. L. et al. (1976), Science, 193, 812-815. [6] Haldemann A. F. C. et al. (1997) JGR, I02, 4097-4106. [7] Haldemann A. F. C. et al. (1997) EOS Trans. AGU, 78 , F404. [8] Ostro (1993) Rev. Mod. Phys., 65, 1235-1279. [9] Harmon J. K. (1998) JGR, 104, 14,065. [10] Harcke L. J. and Zebker H. A. (2000) LPS XXXT, abs no 1770.

Acknowledgments: The research described above was carried out at the Jet Propulsion Laboratory, California Institute of Technology, under a contract with the National Aeronautics and Space Administration.

Table 1. Existing GSSR Mars Radar Data for MER

\begin{tabular}{|c|c|c|c|}
\hline \multirow[t]{2}{*}{ Date } & \multirow{2}{*}{$\begin{array}{c}\text { Lat. } \\
\text { (deg.) }\end{array}$} & \multicolumn{2}{|c|}{ West Longitude (deg.) } \\
\hline & & Rise & Set \\
\hline 14 Sept 1990 & -6.0 & 242 & 289 \\
\hline 22 Sept 1990 & -4.8 & 160 & 214 \\
\hline 29 Sept 1990 & -4.0 & 108 & 131 \\
\hline $2 \mathrm{Oct} 1990$ & -3.8 & 52 & 109 \\
\hline 12 Oct 1990 & -3.3 & 41 & 89 \\
\hline 25 Oct 1990 & -3.6 & 227 & 242 \\
\hline 27 Oct 1990 & -3.7 & 234 & 286 \\
\hline 20 Nov 1990 & -7.6 & 65 & 82 \\
\hline 15 Dec 1990 & -12.0 & 53 & 92 \\
\hline $17 \operatorname{Dec} 1990$ & -12.2 & 23 & 77 \\
\hline $24 \operatorname{Dec} 1990$ & -12.8 & 319 & 20 \\
\hline $28 \mathrm{Dec} 1990$ & -13.0 & 290 & 6 \\
\hline 30 Dec 1990 & -13.1 & 263 & 313 \\
\hline $15 \operatorname{Dec} 1992^{*}$ & 11.0 & 214 & 238 \\
\hline $22 \operatorname{Dec} 1992^{*}$ & 10.0 & 192 & 218 \\
\hline 31 Dec $1992^{*}$ & 8.6 & 158 & 207 \\
\hline 2 Jan 1993* & 8.2 & 150 & 188 \\
\hline $3 \operatorname{Jan} 1993^{*}$ & 8.0 & 90 & 175 \\
\hline 5 Jan 1993* & 7.7 & 19 & 68 \\
\hline $10 \operatorname{Jan} 1993^{*}$ & 6.8 & 15 & 109 \\
\hline 14 Jan 1993 & 6.0 & 308 & 353 \\
\hline 21 Jan 1993 & 5.1 & 239 & 273 \\
\hline
\end{tabular}

\begin{tabular}{cccc} 
23 Jan 1993* & 4.9 & 186 & 238 \\
28 Jan 1993* & 4.4 & 137 & 179 \\
31 Jan 1993* & 4.0 & 99 & 172 \\
3 Feb 1993* & 3.9 & 102 & 168 \\
7 Feb 1993* & 3.8 & 109 & 187 \\
14 Feb 1993* & 3.7 & 8 & 113 \\
\hline
\end{tabular}

* Interferometric data recorded 
THERMAL MODELING OF PI.ANETARY REgOlithS. A. S. Hale' and B. W. Hapke' ${ }^{2}$ ' 'Jet Propulsion Laboratory, 4800 Oak Grove Drive. Pasadena CA 91109 (amy.s.hale@jpl.nasa.gov), ${ }^{2}$ Dept of Geology and Planetary Science, University of Pittsburgh, Pittsburgh PA 15260.

Introduction: The thermal region of the spectrum is one of special interest in planetary science as it is the only region where planetary emission is significant. Studying how planetary surfaces emit in the thermal infrared can tell us about their physical makeup and chemical composition, as well as the ir temperature profile with depth. This abstract will discuss a model of thermal energy transfer in planetary regoliths on airless bodies which includes both conductive and radiative processes while including the time dependence of the solar input function. The applications of this model to the Moon, Mercury, and Io, as well as the rings of Saturn, will be discussed.

The classic model [1] of thermal ene rgy transport in planetary regoliths assumed all heat transfer within a regolith occurred by conduction only, and all radiation occurred at the surface only. However, for bodies without atmospheres, radiation can ciccur between grains of a regolith and can escape iuto space from the immediate subsurface. These processes are important as they can affect the brightness temperature of a body as well as its temperature profile with depth. Models which account for radiative processes, but do not include time dependence were developed first $[2,3]$, and showed that gradients could develop in the topmost layers of a regolith which could affect the position of the Christiansen feature, and which also had implications for the actual temperature of the planetary surface; namely it could possibly be colder than previously thought. This model extended that work by accounting for the time dependence of the solar source function.

The assumptions of our model are as follows: we assume a particulate regoltih with grains close packed and much larger than the wavelength. Particles are assumed to scatter isotropically, and have no wavelength dependent properties (i.e. we assume the regolith is spectrally "gray", with no strong absorption features). We also assume that particle properties do not vary within a defined layer (i.e. properties do not vary continuously with depth). We also treat the visible and thermal parts of the spectrum separately. This model has several free parameters, most of which (single scattering albedo, for example) are fixed by the object. 

A NEW METHOD FOR ESTIMATING THE SINGLE SCATTERING PHASE FUNCTIONS OF REGOLITH GRAINS. P. Helfenstein', 'Center for Radiophysic , and Space Research, 320 Space Sciences Bldg., Cornell University, Ithaca. NY 14853 6801; helfenstein@cuspif.astro.comell.cilu.

Introduction: Hapke's [ 1,2,3] photontretric model has been widely used in solar system remote sensing applications for nearly two decades. Recently, Hapke [4] \& xtended his model to describe the coherent-backscatter opyosition effect and multiple-scattering by particles with anisulropic single particle phase functions (SPPF's). A practical dif ficulty for retrieving Hapke's model parameters from typical planet, satellite, and asteroid photometry data sets is that the nuodel employs a large number of adjustable parameters (at least eight) that can be reliably constrained only for a small nuntber of planetary data sets in which both disk-resolved and whtle-disk observations are available from opposition to very large phase angles [5]. The present work aims to reduce the riumber of adjustable parameters and preserve (or even enhance) the model's accuracy and usefulness by expressing Hapke's parameters in terms of more fundamental physical properties on which they mutually depend. The most difficult part of this task, described here, is to develop a simple method for computing the effective SPPF for structurally complex regolith grains from optical constants, grain-size distribution, and ave ige regolith porosity.

The development of light-s.attering models for irregularly shaped particles is a large, complex subject and many sophisticated methods, such as Discrete Dipole Approximation (DDA) and Monte-Carlo simulations, have been explored elsewhere [6]. Many of these methods are computationally intensive and probably impractical for routine substitution in Hapke's model. Here, I report progress in developing a practical, semi-empirical nethod for estimating the directional scattering behavior (i.e SPPF) of irregular regolith grains. The method employs Optical Transfer Function (OTF) techniques to model how the structural complexity of regolith particles broaden and attenuate th: angular distribution of scattered light relative to that expecterl from ideal spherical particles of equivalent size and composition

Methodology: In solar system remote sensing, OTF filtering is often used as an image processing technique for restoring spatial details in photographs ubtained by imaging cameras. It relies on measurements mate in the engineering laboratory and in-flight of how the actulal light transmission and scattering behavior of a camera's optical system deviates from the predicted behavior for a corsesponding hypothetical ideal camera (one that lacks optical imper fections, internal light scattering, diffraction scattering, etc.) For spatial restoration of images, the OTF is a convolution filter that degrades the theoretical brightness distribution of a scene that would be imaged by an ideal camera to that which is observed by a real camera. In image-processing practice, a picture obtained from an imaging camera is first Fourier transformed, the measured OTF for the camera optical system is then deconvolved, and the result is inverse-Fourier transformed to produce a picture in which spatial detail and photometric fidelity has been restored.

The aim of extending OTF methods to the problem of scattering of light by small, irregularly shaped particles is to provide a simple technique to adjust single particle phase functions (SPPF) computed from Mie theory to realistically approximate the SPPF of an average, naturally occurring regolith grain. A theoretical, perfect spherical grain can be considered as an analog to an ideal camera lens. The angular distribution of scattered light (SPPF) from the ideal spherical particle can be exactly computed from Mie theory given grain size, optical constants, and the wavelength of light [7]. An average regolith grain having the same size and optical constants as the Mie particle is then considered as an analog to a real camera lens. The regolith grain's shape irregularities, internal structure, and packing with other grains are akin to optical defects and diffraction limit of a real camera optical system. A convolution function analogous to the OTF can be found that transforms the Mie scattering phase function to more realistically approximate the corresponding SPPF of the irregular regolith grain. Because SPPF's are most often expanded in terms of Legendre polynomials (for example, to treat multiple-scattering of light among particles $[8,4]$ ), it is advantageous to substitute Legendre transforms in place of the Fourier transforms used in optical engineering.

To derive OTF's for specific examples of rough particles observed in the laboratory, their measured SPPF's and corresponding SPPF's predicted from Mie theory are expanded in Legendre series and a transform filter is found that maps the distribution of Legendre coefficients from the Mie SPPF to those of the rough particle SPPF. Following the analogous approach used in laboratory derivation of OTF's for optical systems, we treat separately each major component of scattering degradation (surface scattering, internal scattering and absorption, and diffraction) by the irregular particle. To first-order, the effects of surface scattering can be isolated from internal scattering and absorption by analyzing linear polarization phase curves for a given irregular particle type and resolving the SPPF contributions from rays scattered parallel and perpendicular, respectively, to the scattering plane. Separate OTF's are then derived for each component of polarization. In cases where it was not possible to accurately measure the diffraction lobe from the rough particle, Allen's [9] approximation for diffraction from rough particles is used to model it. Mie theory describes diffraction for small spherical particles that are isolated and independent. However, the amplitude of the diffraction component in an average regolith grain's SPPF strongly depends on the packing density of particles. Hapke [10] provides the following expression for 
computing the diffraction scattering efficiency, $Q_{n}$, for particles in proximity to one another

$Q_{D}=1-1.209(G \varphi)^{\frac{2}{3}}$ where $G=4 \sigma^{\frac{3}{2}} / 3 \pi^{\frac{1}{2}} v$

$v$ and $\sigma$ are, respectively the volume and geometric crosssection of each grain, and $\phi$ is the packing factor. The ratio of $Q_{D}$ values computed for the irregular grain and Mie particle, respectively, is used to adjust the amplitude of the diffraction contribution from the rough grain prior to extracting the diffraction OTF.

Preliminary Results and Conclusions: Preliminary tests of the OTF method with published intensity and polarization phase curves for rough, irregular particles $[11,12,13]$ are encouraging. The results suggest that, in many cases, satisfactory OTF's for use with Hapke's model can be obtained by simply re-scaling the power spectrum of Mie SPPF's to lower Legendre orders and reducing the amplitude of the rescaled low-order Mie Legendre coefficients to accommodate energy losses due to absorption. This is consistent with the intuitive notion that grain surface irregularities and internal particle structure distribute scattered light over broad ranges of scattering angle.

For the OTF method to be realized in Hapke's model, it will be necessary to conduct a systematic investigation of the Legendre-spectrum scaling rules and their possible dependence on grain-size, optical constants, and particle packing. Ultimately, it is hoped that simple empirical relationships will be found for the scaling rules so that Hapke's current totallyempirical SPPF (i.e. a double Henyey-Greenstein function [1 1,4]) can be replaced by Mie phase functions that have been OTF-filtered to realistically model SPPF's of irregular regolith grains. A special advantage of this approach over the use of the empirical Henyey-Greenstein function is that it provides physically-based a priori information about how a realistic regolith grain scatters light at phase angles that may not be available in planetary photometry data sets from which model parameters are being retrieved. Physically-based modeling of SPPF's in Hapke's equation would greatly reduce the risk of obtaining meaningless solutions for parameters that are retrieved from data sets with limited phase coverage. A wider suite of simultaneous laboratory measurements of polarization and intensity phase curves for isolated, irregular grains of different sizes, compositions, and structural complexity are badly needed to complete this study. Such measurements would be especially valuable if made from the fine particle fraction of returned lunar soil samples [14].

References: [1] Hapke, B.W. (1981) JGR 86, 3039-3054. [2] Hapke, B.W. (1984) Icarus 59, 41-59. [3] Hapke, B.W.
(1986) Icarus 67, 264-280. [4] Hapke, B. (2002) lcarus 157. 523-524. [5] Helfenstein et al. (1997) Icarus 128, 2-14. 16] Mishchenko, M.I., J.W. Hovenier, and L.D. Travis (2000) Light Scattering by Nonspherical Particles: Theory, Measurements and Applications, Acad. Press, San Diego, 690pp. [7] Bohren, C. and D.R. Huffmann (1983) Absorption and Scautering of Light by Small Particles, J. Wiley \& Sons, New York, 530pp. [8] Chandrasekhar, S. (1960) Radiative Transfer, Dover, New York. [9] Allen (1946) Proc. Royal. Astron. London, 106, 137-150. [10] Hapke, B.W. (1999) J. Quant. Specrosc. Radiat. Trans. 61, 565-581 . [11] McGuire A.F. and B.W. Hapke (1995) /carus 113, 134-155. [12] WeissWrana (1983) Astron. And Astrophys. 126, 240-250. [13] Worms et al. (1999) Icarus 142, 281-297. [14] Devine, J.M., D.S. McKay, and J.J. Papike (1982) JGR 87, A260-A268.

Acknowledgement: This study was funded by NASA Grant NAGW-3639. 
The lunar phase curve in The Near Ultraviolet. A. R. Hendrix, Jet Propulsion Laboratory/California Institute of Technology, amanda.hendrix@jpl.nasa.gov.

Introduction: We present resul s from an ongoing program to perform UV measure 1 nents $(215.0$ and $237.0 \mathrm{~nm}$ ) of the Moon at varying silar phase angles to understand the lunar phase curve al ultraviolet wavelengths. We use new observations from the Ultraviolet Spectrometer (UVS) aboard the Student Nitric Oxide Explorer (SNOE) combined with existing observations from the Galileo UVS. The lunar IV phase curve can be used to further understand the scattering properties of the lunar surface. The Moon's scattering properties at visible wavelengths are well understood [1]; studying scattering properties at shortel wavelengths may provide insight into the roles of volume scattering vs. surface scattering and how weathering processes may affect scattering properties [2]. The UV lunar phase curve can also be helpful for UV observers, as the Moon is often used as a UV calibration source, but the UV brightness variation with phase angle has not been well understood.

SNOE UVS Moon Observations: The SNOE satellite has been in Earth orbit since March 1998. It was built and is currently operated at the Laboratory for Atmospheric and Space Physics at the University of Colorado. The primary objective of SNOE is to measure nitric oxide in the Earth's atmosphere, as well as to measure solar soft $x$-rays [3]. The SNOE spacecraft is in a low altitude, polar Earth orbit. The UVS on the spinning spacecraft is in a configuration such that it serendipitously observes the Earth s moon once per month. The angle between the orbital plane and the sun varies with time; this beta angle was initially $\sim 25^{\circ}$. Thus the lunar measurements gener ally occur $1-2$ days before full moon. The offset angle between the orbital plane and the UVS viewing direction can be adjusted, thereby varying the solar phase angle at which the moon is observed. The UVS slit is $0.75^{\circ} \times 0.071^{\circ}$, so as the slit scans over the moon, 7-9 samples are obtained. The UVS has two spectral chanricls, $215.0 \mathrm{~nm}$ and $236.5 \mathrm{~nm}$, each with a bandpass of $3.6 \mathrm{~nm}$. The UVS has made measurements of stellar sources, and calibration of both channels is well understood.

The SNOE lunar data set conists of both serendipitous and dedicated observatiors. The initial moon observations performed by SNOE were purely serendipitous. During the first month of the mission, the moon was observed, $\sim 2$ days before full moon, as SNOE passed over the north pole.

The first dedicated Moon observation was performed in February of 2000. The observational timing was changed during the nighttime portion of the orbit so that, rather than scanning the limb, the UVS scanned the portion of the sky (in the zenith direction) where the Moon was projected to be, based on the knowledge of the Moon's right ascension and declination. Other dedicated lunar observations have been performed, to observe the Moon at a smaller phase angle than the orbital beta angle.

The solar phase angle during each Moon observation is determined using data from the Solar Angle Sensor on the Solar X-Ray Photometer, which measures the angle between the sun and the viewing direction of the instruments.

Galileo UVS Moon Observations: To increase the phase angle range of the Moon observations, we have combined the SNOE data with data from the Galileo UVS encounters with the Moon. The phase angle range from the Galileo flybys is $\sim 19^{\circ} \cdot 70^{\circ}$, providing some overlap with the SNOE data set in addition to valuable larger phase angle coverage. The Galileo UVS F-channel covers the $200-320 \mathrm{~nm}$ wavelength range; in this analysis we use only the data from the ranges covered by the SNOE UVS channels.

Analysis: The phase angle range of the current data set is $3.5-70^{\circ}$. We model the data using the Hapke model (see the figure below) to derive a determination of the ultraviolet photometric parameters of this relatively well-understood surface. These Hapke parameters can then be used for comparison with visible results to understand changes in the scattering properties of the surface with decreasing wavelength.

References: [1] Helfenstein, P. and J. Veverka (1987) Icarus, 72, 342-357. [2] Henry, R. C., W. G. Fastie, R. L. Lucke, B. W. Hapke (1976) Moon, 15. 51-65. [3] Barth, C. A., S. M. Bailey, S. C. Solomon. (1999) GRL, 26, 1251-1254. [4] Hendrix, A. R. (1996) Ph.D. Thesis.

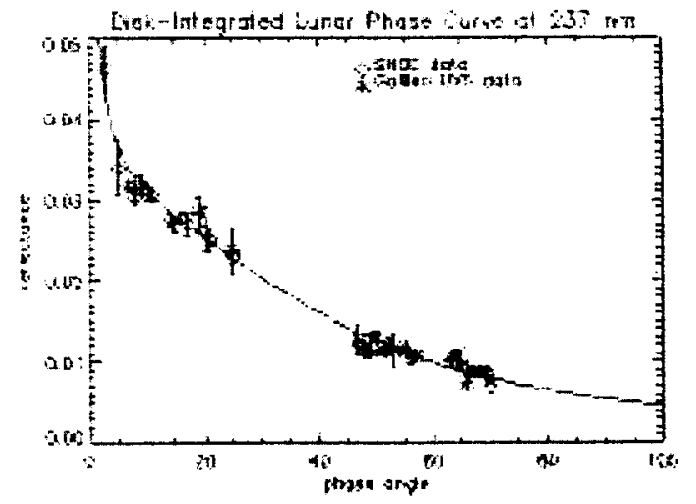



Ultraviolet Surface Properties of the Icy Galilean Satellites from Phase Curve Analysis. A. R. Hendrix and D. L. Domingue ${ }^{2}$, 'Jet Propulsion Laboratory/California Institute of Technology, 4800 Oak Grove Dr., Pasadena CA 91109, amanda.hendrix@ipl.nasa.gov, Johns Hopkins University/Applied Physics Laboratory, Johns Hopkins Rd., Laurel MD 20723, deborah.domingut@jhuapl.edu

Introduction: In this study, we use ultraviolet observations from the International Uilraviolet Explorer (IUE) and the Galileo Ultraviolet Spectrometer (UVS) to compose the ultraviolet solar phase curves of the icy galilean satellites. Broadband rotation phase curves from 0.26 to 0.32 microns are constructed in order to examine the rotational behavior of the icy Galilean satellites in the ultraviolet. After normalizing the rotational variations, modeling of the solar phase variations are compared to comparable studies in the visible.

Background: Studies of the rotational phase curve variations at visible wavelengths $\mathrm{for}$ the icy Galilean satellites indicate distinctive albedo changes from leading to trailing hemisphere. Processes such as the preferential bombardment of the leading hemisphere by micrometeorites and/or the preterential bombardment of the trailing hemisphere by co-rotating ions within Jupiter's magnetosphere have been proposed as mechanisms for creating these albedo variations. Ultraviolet observations by IUE [1] and the Hubble Space Telescope (HST) have measured absorption features attributed to surface chemistry resulting from ion interactions with the surface.

Discovery observations with IUI: in the late 1970's showed an absorption feature at 0.18 microns on Europa whose strength correlated witl the albedo variations observed on this satellite, and whose source was proposed to be the implantation of magnetospheric sulfur ions into the water-ice surface [2]. Later measurements with HST [3] were comprared to laboratory spectra of irradiated water-ice and $\mathrm{SO}_{2} / \mathrm{H}_{2} \mathrm{O}$ layered frosts [4], to show that the observecl spectral feature is better matched by the layered frosts, implying an endogenic origin.

Ganymede's rotational albedo varriations are similar to Europa's, with the leading hemisphere being generally brighter than the trailing hemisphere. Ganymede's trailing hemisphere, however, has a different absoption feature than Europa's. Ganymede's absorption feature has been found to be ozone, and is most concentrated in the trailing hemisphere polar regions and at large solar zenith angles., commensurate with production by ion bombardment and destruction by solar photon interactions $[5,6]$. HST visible observations of Ganymede have also detected oxygen on the trailing hemisphere at low latitudes, consistent with production via ion bombardment processes [7].
Callisto's rotational albedo varations are opposite to those seen on Europa in the visible, where the leading hemisphere is brighter than the trailing hemisphere. This is also the case in the ultraviolet. IUE and HST observations in the ultraviolet have also detected a 0.28 micron absorption featuer, linked to $\mathrm{SO}_{2}[8,9]$. This absorption feature shows no correlation to the rotational albedo variations, however the IUE observations show some spatial correlation to possible neutral wind interactions suggestive of neutral sulfur implantation into Callisto's water-ice surface. The strongest 0.28 micron absorption seen in the IUE data was in the region containing Valhalla basin. Galileo UVS also detected this absorption feature on the leading hemisphere, but found no definitive correlation with surface feature [10].

Data Set and Analysis: Rotational phase curves were derived for each satellite for each wavelength bin $(0.26,0.27,0.28,0.29,0.30,0.31$ and 0.32 microns), using IUE data in the $10^{\circ}$ phase angle range. These rotational phase curves were then used to correct the entire data set (including additional solar phase angles) for each satellite to particular longitudes for photometric analysis. The Europa data set was the most extensive and allowed us to analyze all four longitude regions: the leading and trailing hemispheres, as well as the Jovian and anti-Jovian hemispheres. The Ganymede and Callisto data sets were more limited; for these satellites, we used the rotational phase curves to correct to $90^{\circ} \mathrm{W}$ longitude, and derived a global set of photometric parameters. This global set of photometric parameters was also derived for Europa, for comparison with Ganymede and Callisto.

Results: In this presentation, we discuss the rotational and solar phase curves we have derived for the three icy galilean satellites. We have fit Hapke photometric models to the solar phase curves for Europa, Ganymede and Callisto, and to four longitude regions on Europa. We intercompare these to look for variations related to differences in exogenic processes as discussed above. Although the data sets are not ideal the data are sometimes noisy and phase angle coverage tends to be sparse, this work represents the first indepth ultraviolet analysis of the photometric parameters of the icy galilean satellites, and the results are useful for understanding variations in scattering phenomena with wavelength. 
ICY GALILEAN SATEllite PHASE CURVE ANALYSIS: A. R. Hendrix and D. L. Domingue

References: [1]Nelson et al., 1987, Icarus 72, 358380. [2] Lane et al, 1981, Nature 292, 38-39. [3] Noll et al., 1995, J. Geophys Res. 100, 19057-19059. [4] Sack et al. 1992, Icarus 100, 534-540. [5] Hendrix et al., 1999, J. Geophys. Res. 104, 14169-14178. [6] Noll et al., 1996, Science 273, 341-343. [7] Spencer et al.,
1995, J. Geophys. Res. 100, 19049-19056. [8] Lane and Domingue, 1997, Geophys. Res. Lett. 24, 1143 1146. [9] Noll et al., 1997, Geophys Res. Lett., 24, 1139- 1142. [10] Hendrix et al., 1998, LPSC XXIX. 
EARTH-BASED RADAR SPECKLE DISPLACEMENT INTERFEROMETRY TO STUDY THE SPINVECTOR OF VENUS. I. V. Holin. Space research institute, Moscow, Russia, holin@ mail.cnt.ru

Introduction: The spin-vector of Venus $\mathbf{\Omega}$ was investigated by various Earth- and spacecraft-based techniques [1-3] (see also refs. in [3]) but until now no experimental data have been olytained on variations of $\Omega$ both in magnitude and direction because of insufficient accuracy and too long neasuring interval (much longer than the period of viriations). In this work a new on principle ground-based radar interferometric technique named Radar Speckle Displacement Interferometry (RSDI) is proposed to measure precisely instantaneous vector components of $\boldsymbol{\Omega}$ and their variations with time.

The essentials and theory of RSDI were developed in [4-6] (see also refs. in [6]). The technique is based on a so called far coherence (speckle displacement) effect for speckled radar fields scattered from randomly rough surfaces of moving objects and aims at precise measurement of their instantaneous rotational-progressive motion parameters.

Far Coherence or Speckle Displacement effect: Let us illuminate Venus from Goldstone (USA) by a monochromatic radiowave at wavelength $\lambda=13 \mathrm{~cm}$ (Goldstone's $3.5 \mathrm{~cm}$ is absorbed by the Venus' atmosphere). The randomly rough surface of Venus can be considered as a reflecting antenna with a random speckled backscattering pattern. The average size of a speckle near Earth will be $\mathrm{l}=\lambda \mathrm{R} / \mathrm{a}, \sim 3.5 \mathrm{~km}$, where $\mathrm{R} \sim 40$ million $\mathrm{km}$ - the Earth-Venus distance at inferior conjunctions, $a_{1} \sim 1500 \mathrm{~km}-$ the effective size of subradar reflecting area in the direction orthogonal to the line-of-sight. The Fraunhofer pattern does not depend on distance while the Fresnel pattem changes with the distance to Venus. When Venus rotates with respect to Earth all its Fresnel speckle pattern range layers rotate as well remaining "freezed" for a rather long time. One of these "freezed" speckle layers corresponding to the current Earth-Venus distance sweeps across the Earth surface. It was shown in [4] that the speckle pattern displacement in a "freezed" state can be characterized by a length $\mathrm{L}=2 \mathrm{R}\left(\mathrm{N} / \mathrm{a}_{\mathrm{T}}\right)^{0.5}$ $\sim 130000 \mathrm{~km}$, where $a_{\mathrm{r}} \sim 50 \mathrm{~km}$ - the effective size of subradar reflecting area along the line-of-sight. It follows from $\mathrm{L} \gg 12800 \mathrm{~km}$ (the diameter of Earth) that the speckle pattern remains "freezed" while its displacement all over the surface of Earth. In other words high correlation can be observed between radar echoes received at opposite sides of Earth separated by thousands $\mathrm{kms}$ and this effect can be referred to as far coherence of scattered speckled radar fields [7-9].
RSDI: The main principle of RSDI as applied to a planet's rotation measurement is as follows. If two antennas are placed along with the speckle displacement direction they can see the same speckles passing through them and cross correlation of the received echoes will give the velocity $v$ and direction $v / v$ (the receiving baseline orientation changes with time slowly due to rotation of Earth) of speckle displacement related to the transversal (orthogonal to the lineof-sight) vector component $\boldsymbol{\Omega}_{\text {, }}$ of the planet's instantaneous spin-vector $\Omega$ [4-15]. It was shown in [5] that the above RSDI-principle coincides with the procedure of optimum processing of scattered random speckled radar fields under monochromatic illumination and the minimum of measuring time to estimate instantaneous rotational-progressive motion parameters and their fluctuations with time. This means that RSDI may reach the limit accuracy of planet's instantaneous spin-components estimation when the measuring time is no more than a few minutes and there exists no another Earth-based radar interferometer which could have accuracy better than RSDI in these conditions.

RSDI accuracy: Let us calculate the expected one-look accuracy for the radar interferometers Goldstone-Green Bank (USA) and Evpatoria (Crimea, Ukraine)-Effelsberg (Bonn, Germany)-Bear Lakes (Moscow, Russia) in Europe $(\lambda=6 \mathrm{~cm}$, values are given below in brackets) in accordance with the methodology $[5-10,12,13]$. The potential limit of $\boldsymbol{\Omega}_{\mathrm{t}}$ estimation was derived in [5] where it was shown using the Maximum Likehood criterion that the limit one-look relative accuracy $\sigma$ of $\Omega_{1}$ magnitude (index 1) and direction (index 2) estimation by a twoelement radar interferometer can be expressed analytically as

$$
\sigma_{1,2}=\frac{l_{v .0}}{q b} \cdot \frac{v}{v_{\Omega}} \sim 10^{-6} \quad\left(-1.5 \cdot 10^{-6}\right)
$$

where the baselength $b \sim 3000 \mathrm{~km}(\sim 2000 \mathrm{~km}), I_{v}$ is used for $\sigma_{1}$ and $l_{0}$ for $\sigma_{2}, l_{v} \sim l_{a} \sim 1$ due to nearly spherical shape of Venus, $v_{\Omega}$ is a part of $v$ caused by rotation only (under $\mathrm{V}=0$ ), $\mathrm{q}=\mathrm{Q}^{0.5}, \mathrm{Q}$ - power signal-to-additive noise ratio (snr) at the correlator output, defined as

$\mathrm{Q}=\mathrm{N} /\left(1 / \mathrm{Q}_{1}+1 / \mathrm{Q}_{2}+1 / \mathrm{Q}_{1} \mathrm{Q}_{2}\right) \sim 7 \cdot 10^{5}\left(-1.7 \cdot 10^{5}\right)$ 
$\mathrm{N}=\mathrm{FT} \sim 450(\sim 450), \quad \mathrm{T} \sim 30 \mathrm{~s}(\sim 14 \mathrm{~s})-$ correlator integration time, $Q_{1,2}$ - power snr at the correlator input within Doppler bandwidth $\mathrm{F} \sim 15 \mathrm{~Hz}(\sim 32 \mathrm{~Hz})$ for Green Bank or Effelsberg (index 1) and Goldstone or Evpatoria/Bear Lakes (index 2), $\mathrm{Q}_{1}=\mathrm{P}_{1} / \mathrm{P}_{\mathrm{n}}$ $4600(\sim 1060), Q_{2}=P_{2} / P_{n}-2300(-535)$, noise power $P_{n}=k_{B} T_{s} F \sim 5.4 \cdot 10^{-21} \mathrm{~W}\left(\sim 2.3 \cdot 10^{-20} \mathrm{~W}\right)$, $T_{S} \sim 25 \mathrm{~K}(\sim 50 \mathrm{~K})$ - system temperature, $\mathrm{k}_{\mathrm{B}}-$ Boltzmann's constant, from the radar equation [3]

$$
P_{1,2}=\frac{P_{i} \cdot G_{1,2} \cdot G_{2} \cdot \lambda^{2} \cdot S}{(4 \pi)^{3} \cdot R^{4}}
$$

$P_{1} \sim 2.5 \cdot 10^{-17} \mathrm{~W}-$ echo power at Green Bank, $P_{2} \sim$ $1.2 \cdot 10^{-17} \mathrm{~W}-$ echo power at Goldstone, $P_{t} \sim 450 \mathrm{~kW}$ $(\sim 100 \mathrm{KW})$ - transmitted power, $\mathrm{G}_{1} \sim 1.8 \cdot 10^{6}(\sim$ $\left.8.4 \cdot 10^{6}\right)$ Green Bank (Effelsberg) antenna gain, $\mathrm{G}_{2} \sim$ 9.1 $10^{5}\left(-4.3 \cdot 10^{6}\right)$ - Goldstone (Evpatoria/Bear Lakes) antenna gain, $S-10^{7} \mathrm{~km}^{2}$ - Venus' radar cross section. Eq. (1) is consistent with the well known results for time delay estimation of random signals [5].

The optimum processing algotithm leading to the limit accuracy Eq. (1) is too complicated and a quasioptimum one can be used in practice. Computer simulation showed that loss in accuracy while this transition is $25 . .30 \%$ [6] and instead of the numerical values in Eq. (1) we will have

$$
\sigma \sim 1.3 \cdot 10^{-6}\left(-2 \cdot 10^{-6}\right)
$$

Values (2) mean that one-look (for -1 min observation) accuracy in determination of Venus' polar axis orientation as projected onto a transversal plane orthogonal to the instantaneous line-of-sight with one of the above two-element interferometers will be $300 . . .400$ mas (milliarcseconds). If one interferometer is used $\mathbf{n}$ times (=days) within a single conjunction the accuracy can be $\sim \mathrm{n}^{0.5}$ times better. With $\mathrm{m}$ comparable interferometers accuracy will be another $\mathrm{m}^{0.5}$ times higher. For $n=16$ and $m=4$ the resulting accuracy can be as fine as $40 . .50$ mas (!). It is not easy to achieve these limits even through space missions to the planet. In addition variations of Venus' polar axis (if present) can be studied. The resulting relative accuracy in rotational velocity of Venus can approach $2 \cdot 10^{-7}$ again with the possibility to study variations. RSDI-observations during several successive inferior conjunctions may lead to a few tens of mas in polar axis orientation and $-10^{-7}$ in rotational velocity. More powerful transmitters should give further improvements.
Experiments: The effect of far coherence (speckle displacement) discovered theoretically in [4,5] is now proven in fact both in Europe [15] and in the USA $[16,17]$. The RSDI data obtained on Mercury in accordance with $[4,5,10,11]$ in the USA in May-June 2002 [17] confirm the theory developed in [4-15] in main part and are now processed to determine Mercury's obliquity and librations [18]. RSDI accuracy is expected to be several orders of magnitude better than that obtainable through other Earthbased radar techniques [5-12,14,15] and can be comparable [19] with the accuracy of the two upcoming space missions to Mercury: MESSENGER (USA) and BepiColombo (Europe-Japan).

RSDI experiments on Venus based on the above theory are possible both in the USA and in Europe as early as this 2002 fall. Further opportunities for maximum precision measurements will take place in spring/summer 2004 while the next inferior conjunction of Venus with Earth. Future RSDI experiments can be aimed at substantial improvement in knowing the Venus' polar axis orientation in the sky and precise estimation of the spin-vector variations with time both in magnitude and orientation.

References: [1] Davies M. E. et al. (1992) $J$. Geophys. Res. 97, 12141 [2] Shapiro I. I. et al. (1990) Astron. J. 100, 1363 [3] Ostro S. J. (1993) Rev. Mod. Phys. 65, 1235-1279. [4] Holin I. V. (1988) Radiophys. Quant. Electron. 31, 515-518. [5] Holin I. V. (1992) Radiophys. Quant. Electron. 35, 433-439. [6] Holin I. V. (1998) Uspekhi Sovr. Radioel., 4, 3-15 (in russian). [7] Holin I. V. (2002) Solar System Res. 36, 226-233. [8] Holin I. V. (2002) Meteorit. Planet. Sci. 37, No. 9 (submitted). [9] Holin I. V. (2002) Proceedings of the IEE Radar 2002 conf., UK (in press). [10] Holin I. V. (1999) Uspekhi Sovr. Radioel., 7, 1628 (in russian). [11] Holin 1. V. (2001) Mercury conference (abstract \# 8038). [12] Holin I. V. (2002) LPS XXXIII, 1387+. [13] Holin I. V. (2002) LPS XXXIII, 1393+. [14] Holin I. V. (2002) Met. Soc. 65, 5041t. [15] Holin I. V. (2002) Met. Soc. 65, 5131+. [16] Peale S. J. (2002) private communication. [17] http://www.gps.caltech.edw/margot/mercury/

[18] Margot J-L. (2002) private communication [19] Peale S. J. et al. (2002) Meteorit. Planet. Sci. 37, No. 9 (submitted). 
EVIDENCE FOR GLOBAL RADAR SPECKLE PATTERN DISPLACEMENT TO MEASURE MERCURY'S OBLIQUITY AND LIBRATIONS. I. V. Holin, Space Research Institute, Moscow, Russia, holin@mail.cnt.ru

Introduction: Planetary rotations and related parameters have been measured throinghout years by radar and optical imaging techniques remotely from Earth or spacecraft. It is well understandable from our everyday life that spin components of rotating bodies can be estimated by obtaining their images at different moments of time and then comparing them to each other. Our eyes employ this principle for thousands years with Sun as a source of illumination. Note that Sun is an incoherent source while laser or radar are coherent. At what extent the imaging approach is good in these "coherent" conditions and if there exists a better approach? An answer was given in $[1,2]$.

Theory: A new approach does not necessarily lead to a substantial improvement in accuracy but to improve accuracy by several orders of magnitude as a rule one must use a new physical principle. As applied to planetary rotation measurements by Earthbased radar a new principle can be based on a so called "freezed" speckle pattern displacement effect or simply speckle displacement effe:t (SD-effect) [1]. Let us illuminate Mercury by a munochromatic radiowave at wavelength $\lambda=3.5 \mathrm{~cm}$ Due to coherent illumination and rough surface of Mercury the backscattering pattern is speckled like those well known and easy observable in laser optics experiments [3]. The mean effective transversal (orthogonal to the line-of-sight) size of a speckle near Earth is $l_{t} \sim \lambda R / a_{t}$ $\sim 3 \mathrm{~km}$, where the Earth-Mercury approach $\mathbf{R} \sim 90$ million $\mathrm{km}, \mathrm{a}_{\mathrm{t}} \sim 1000 \mathrm{~km}-$ the effective transversal diameter of subradar backscattering area on Mercury. The mean radial (along the line-of-sight) size of a speckle can be estimated as $1_{r}-l_{1}{ }^{2} \lambda-260,000 \mathrm{~km}$ characterizing the thickness of transversal speckled layers inside which the Fresnel backscattering pattern does not change with range.

What happens with the speckle pattern when the object rotates was considered in [4] for a particular case of cylinder rotation with the spin-axis orthogonal to the line-of-sight. For arbitrary rotationalprogressive (spin-orbital) motion alld arbitrary shape of an object the problem was solved in [1] where it was shown that in the most general case excluding some theoretical geometries and nutions nearly impossible in practice of Earth-based radar astronomy the reflected speckle pattern first sweeps across the receiving aperture plane in a nearly "freezed" state for a rather long distance $\mathrm{L} \sim 2 \mathrm{R}\left(\lambda \mathrm{a} \mathrm{a}_{\mathrm{r}}\right)^{0.5} \sim 150,000$ $\mathrm{km}$, where for Mercury $\mathrm{a}_{\mathrm{r}} \sim 50 \mathrm{~km}$ - effective radial size of the subradar backscattering area, and then decorrelates (decorrelation starts immediately but becomes visible after the speckle pattern overcomes the distance $\mathrm{L}$; also there are particular geometries free of decorrelation, e.g. when the transmitter is placed at the center of the receiving baseline). $L$ is much longer than the diameter of Earth so the speckle pattern while its displacement in any case must remain "freezed" all over the Earth's surface. If this "freezed" SD-effect really exists in the global Earth scale than the correlation of radar echoes obtained at the two antennas, which form a two-element interferometer with the baseline that due to rotation of Earth can be instantaneously colinear with the SD-direction, can provide two observables, the time delay and the epoch assotiated with maximum correlation, both of which may constrain Mercury's spin state to accuracy as high as one part in 100,000 (a few arcseconds) [513].

Experiments: To verify the SD-effect in practice of radar astronomy a number of radar interferometric experiments were proposed in Europe [5,6,12], EuroAsia $[2,5,6]$ and in the USA [5-13]. Altemative radar techniques, e.g. repeat orbit radar interferometry $[14,15]$ were proposed and discussed as well (for comparison of the techniques see [16]). During a year long discussion of the above SD-approach with S. J. Peale he organized a group of radar astronomers who successfully carried out the Goldstone - Green Bank SD-experiment on Mercury in May-June 2002 [17]. The theory and potential limits of SD-experiments including Goldstone - Green Bank can be found in $[8,9,12,13]$. A large cross-correlation between Green Bank and delayed Goldstone radar echoes was clearly detected in accordance with the above theory at least on two days: on May 22 and June 02 . One of these cross-correlation peaks caused by the SD-effect is shown in [17]. It follows from the existence of the peak that the effect of "freezed" speckle pattern displacement discovered theoretically in $[1,2]$ is now proven in fact over the baselength $\sim 3000 \mathrm{~km}$ and further Goldstone - Green Bank data processing (now in progress) may lead to the first in history (!) determination of Mercury's obliquity and librations as discussed in $[7-9,12,13]$.

Discussion: The intermediate experimental result [17] allow estimation of the expected accuracy which can be compared with the potential limits [2,7$9,12,13]$. In fig. 1 a correlation graph is shown sche- 
maticly with necessary parameters estimated from the experimental graph [17] (a periodic disturbance of the amplitude $\sim 0.07$ with the period $\sim 15 . .20$ times larger than the width of the correlation peak seen in the graph [17] was removed).

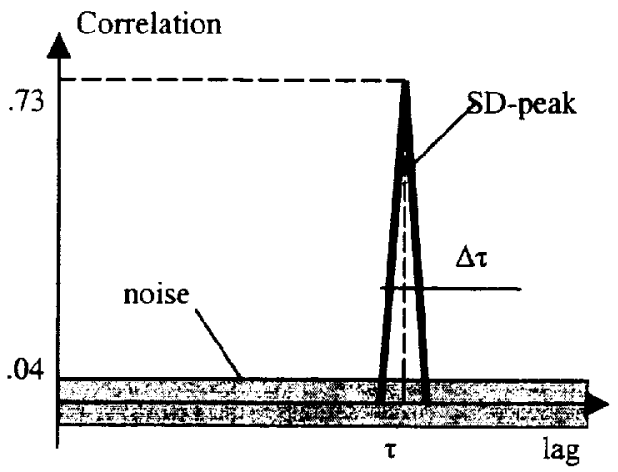

Fig. 1. Goldstone - Green Bank crosscorrelation caused by the SD-effect

As it follows from the theory [1-2,5-13] the maximum of the Goldstone - Green Bank crosscorrelation peak should take place at the time delay $\tau$ $\sim 10 \ldots 13 \mathrm{~s}$ needed by the speckles to come from Green Bank to Goldstone and its width can be expected within $\Delta \tau \sim 10 \ldots 15 \mathrm{~ms}$ (both parameters were not given in [17]). The maximum value of cross correlation is $\sim 0.73$ and the noise amplitude is $\sim 0.04$ (fig. 1) so the signal-to-noise ratio is $q \sim 18$ and this may lead to the relative accuracy $[2,5-13]$ in instantaneous rotational velocity $\sigma_{1} \sim \Delta \tau / q \tau \sim 610^{.5}$ and in obliquity $\theta \quad \sigma_{2} \sim 12$ arcsec. The obliquity itself can be expected to be as small as a few arcminutes [16] so the relative accuracy in $\theta$ will be $\sim 10 \%$ (for $\theta \sim 2$ arcmin). The physical libration amplitude $\varphi$ is expected within $20 . .60$ arcsec [16] so the one look relative accuracy in $\varphi$ in accordance with the methodology $[9,13]$ will be $\sim 50 \%$ (for $\varphi \sim 30$ arcsec). So already the intermediate result [17] is outstanding allowing the first in history measurement of $\theta$ with good accuracy. At the same time potential limits for the May-June 2002 Goldstone - Green Bank conditions are $\sigma_{1} \sim 10^{-5}$ and $\sigma_{2} \sim 2 \operatorname{arcsec}[9,12,13]$ and we all do hope that further quasioptimum processing of the echoes will reach these limits allowing determination of both $\theta$ and $\varphi$ (in case of damped free libration [16]) with fractional uncertainties $\sim 3 \%$ and $\sim 15 \%$ respectively.
Acknowledgments: It is a pleasure to thank S. J. Peale for discussion of many aspects of this work.

References: [1] Holin I. V. (1988) Radiophys. Quant. Electron. 31, 515-518. [2] Holin I. V. (1992) Radiophys. Quant. Electron. 35, 433-439. [3] J. Dainty, ed. (1978) Laser speckle and related phenomena, Springer Verlag. [4] George N. (1976) JOSA 66, 1182. [5] Holin I. V. (1998) Uspekhi Sovr. Radioel., 4, 3-15 (in russian). [6] Holin 1. V. (1999) Uspekhi Sovr. Radioel., 7, 16-28 (in russian). [7] Holin I. V. (2001) Mercury conference (abstract \# 8038). [8] Holin I. V. (2002) LPS XXXIII, 1387+. [9] Holin I. V. (2002) Solar System Res. 36, 226-233. [10] Holin I. V. (2002) Met. Soc. 65, 5041+. [11] Holin I. V. (2002) Met. Soc. 65, 5131+. [12] Holin I. V. (2002) Proceedings of the IEE Radar 2002 conf., UK (in press). [13] Holin I. V. (2002) Meteorit. Planet. Sci. 37, No. 9 (submitted). [14] Slade M.A. et al. (2001) Mercury conference (abstract \# 8062). [15] Slade M.A. et al. (1999) LPS XXX, 1143+. [16] Peale S. J. et al. (2002) Meteorit. Planet. Sci. 37, No. 9 [17] http://www.gps.caltech.edw/ margot/mercury/ 
USING THE HAPKE ROUGH REFLECTANCE FACTOR FOR CALIBRATION OF ATMOSPHERIC EMISSIONS AT MERCURY AND THE MOON. Donald M. Hunten and Ann L. Sprague, Lunar and Planetary Laboratory, University of Arizona, 1629 E. University Blvd. Tucson, AZ 85721 - 0092. dhunten@lpl.arizona.edu, sprague@Ipl.arizona.edu.

Introduction: Emission lines of atmospheric constituents of the Moon and Mercury (especially sodium and potassium) must be absolutely calibrated before the abundances can be obtained. It is convenient to use the intensity of the surface at an adjacent wavelength as the standard, and to use Hapke theory to obtain this intensity. This procedure and some of its pitfalls are discussed here.

Methods Used: Observations of these emission lines in the presence of a strong background from the surface must be made at high spectral dispersion. In spectra of Mercury both lines and continuum are present and the intensity of the light from the surface can be obtained simply by use of a wavelength nearly adjacent to the emission line. The lunar atmosphere is usually observed above the bright limb. Although there is also a strong component of light from the surface, scattered into the instrument by the Earth's atmosphere, it cannot be used for an iutensity calibration. Instead a second exposure is taken, with the telescope pointed directly at the surface. The intensity from this region may then be computed if the Hapke parameters have been obtained and tabulated. For Mercury we use average parameters for the whole surface, taken from the review chapter by Veverka et al. [1]. We compute an array of rough reflectances $r_{R}$ and then an array of intensities for the entire illuminated area of the planet. These can then be used to compute a synthetic image of the planet, smeared by various assumed amounts of atmospheric "seeing". Cuts are then taken across these images at the location of the spectrograph slit, and the one that fits best selected. The procedure is described in detail by Sprague et al. [2], who find that the 2dimensional Gaussian used to repre sent the seeing has, in one example, a half-with parameter of 1.5 arcsecond. This large value is the result of observing the planet close to the horizon. Another group [3] uses similar procedures, though they have not been described in as much detail.
Discussion: A major advantage of the method described is that the calibration is obtained (for Mercury) under the same geometrical and sky conditions as the measurement; for the Moon they are very nearly the same, differing only by the time taken to move the telescope. The accuracy does depend on the photometry from which the Hapke parameters were obtained, and on the accuracy with which these parameters represent the data.

Intensities computed with Hapke theory tend to be brighter near the backscatter direction than those observed. If the instrument is calibrated under this condition, it will appear to be less sensitive than it really is. The sodium data set of Potter and Morgan [4], which was taken over a wide range of lunar phase angle, showed a marked minimum of intensity near full phase. The suggestion of these authors was that the location of the Moon in the geomagnetic tail shielded its surface from sputtering by solar-wind ions. But a later measurement [5] by a different method, calibrated against a source inside the instrument, did not find any diminution of intensity compared with what was found at smaller phase angles. It seems possible that the result of [4] stemmed from a computed surface intensity that was too bright near the full phase. A useful remedy would be to check the derived calibration factors of the instrument, which should normally be stable over periods of days to months unless there are big changes in sky conditions..

References: [1] Veverka, J., Helfenstein, P., Hapke, B. and Goguen, J.D. (1988) in Mercury, eds. F. Vilas, C.R. Chapman and M.S. Matthews (Tucson, Univ. of Arizona Press), 37 - 58. [2] Sprague, A.L., Hunten, D.M. and Grosse, F.A.. (1996), Icarus 123, 345 - 349. [3] Killen, R.M., Potter, A.E. and Morgan, T.H. (1990), lcarus 85, 145 - 167. [4] Potter, A.E. and Morgan, T.H. (1994) GRL 21, 2263 - 2266. [5] Mendillo, M., Baumgardner, J. and Wilson, J. (1999). Icarus 137, 13-23. 

DUST COATINGS AT THE MARS PATHFINDER LANDING SITE. J.R. Johnson', W.M. Grundy', M.T. Lemmon ${ }^{3},{ }^{1}$ U.S. Geological Survey, 2255 N. Gemini Dr., Flagstaff, AZ 86001, ${ }^{2}$ Lowell Observatory, Flagstaff, AZ, ${ }^{3}$ Texas A\&M University, College Stition, TX, jrjohnson@usgs.gov.

Summary: Multispectral imaging of the Mars Pathfinder (MPF) radiometric calibration targets (RCTs) by the Imager for Mars Pathfinder (IMl') camera provided sufficient data to model the photometric changes associated with variable deposition of atmospheric dust on the RCTs. Sky models were used to correct for variations in diffuse/direct illumination conditions during the mission, and two-layer Hapke radiative transfer models were developed to constrain variations in spectral properties associated with dust deposition on the RCTs. The results are consistent $w$ ith dust deposition during the MPF mission amounting to dust optical depth (tau) values $<0.5$ (i.e., $<10$ um effective thickness of $10-20 \mu \mathrm{m}$ particles for porosity 0.80 ), although variability in the dust thickness with time may result from erosive/depositional phases of aeolian activity.

Background: The IMP camera was a multispectral stereo imaging system covering the $440-1000 \mathrm{~nm}$ region in 12 wavelengths. Two RCTs were placed on the MPF lander to provide a direct reference for radiometric and color calibration of IMP images (Figure 1) [1] . All surfaces at the MPF landing site were subject to dust deposition from the atmosphere throughout the 83Sol mission. Images of the RCTs were acquired regularly to assist in calibration of images to relative reflectance. Contamination of the RCJ's with dust could therefore influence image calibration. Radiative transfer models of sky illumination that incorporate opacity measurements have been developed to help compensate for variations in illumination and the relative contributions of diffuse and direct skylight on both scene and RCT images [2-5]. Two-layer radiative transfer models of dust-coated surfaces have been developed to distinguish dust and substrate spectral characteristics and dust thickness [e.g., 6].
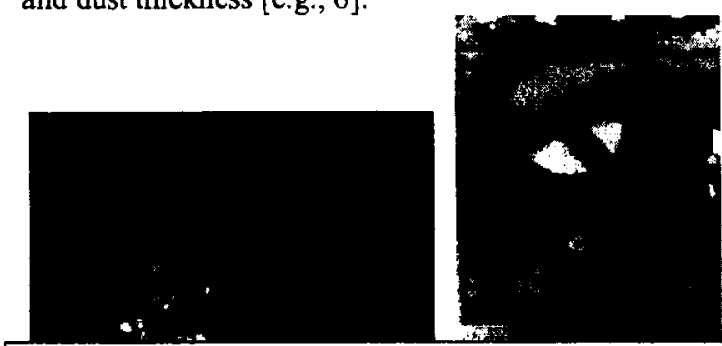

Figure 1. (a) IMP image of lower radiometric calibration target (RCT) showing shadow post, white, gray, and black rings, and color chips; (b) IMP image of the upper RCT, (spacecraft cabling blocking portion of view).

Methodology. We initially approached this problem using images of the RCTs acquired early in the mission
(Sol 3) to calibrate to relative reflectance all RCT images taken at nearly the same local solar time (LST) throughout the mission. Using this approach we demonstrated that dust indeed coated the RCTs and reddened its spectrum with time [7]. However, we treated these as preliminary results owing to the effects of scattered light from the lander and ongoing improvements made to the sky and two-layer models. Our current approach used calibrated radiance data from the RCTs in combination with improved radiative transfer models. We again used the Sol 3 observations as a baseline to observe later variations. Knowing the photometric properties of the RCTs from laboratory measurements [1], we used the sky model to determine the expected radiance of the targets under Mars illumination conditions. These values were compared to data extracted from calibrated radiance images of the targets. Outliers between modeled and observed data found to result from observational problems (e.g., dropped image data, IMP cabling in the field of view; Figure 1) were removed from the dataset. The resulting data set was provided to the two-layer model.

Two-layer model. We used a two-layer version of the Hapke model [8, section 9.D.3] to compute bidirectional reflectance for both the clean substrate (from lab measurements) and RCTs throughout the mission. We first studied cases where dust accumulation was assumed to be linear as a function of time over the duration of the mission. Accumulation rates derived from these models typically ranged from 0.001 to 0.002 optical depths per sol. For dust grain sizes between 5 and $20 \mu \mathrm{m}$ and $80 \%$ porosity, these tau rates correspond to mean accumulation of 0.02 to $0.1 \mu \mathrm{m}$ per sol. We ran models with isotropic single scattering phase functions as well as with phase functions based on atmospheric dust scattering properties [e.g., 2-4]. For some runs, we used dust absorption coefficients determined for palagonites from previous studies [6,9], while in others we left the dust single scattering albedos as free parameters. We also ran test cases where dust thickness for each sol was a free parameter, rather than assuming a simple linear accumulation with time.

Results. Figure 2 shows representative observed spectra of the white RCT ring on Sols 3, 42, and 83 compared to model results using an isotropic phase function for constant dust accumulation rate. A decrease in $443 \mathrm{~nm}$ reflectance values with time resulting from dust deposition is apparent. Sol 83 model values fit more poorly, potentially due to greater variability in 
atmospheric opacity compared to earlier in the mission [2-4]. Figure 3 shows results from the same model as a function of time, where the RCT white ring's $443 \mathrm{~nm}$ reflectance decreases with time and the black ring's $671 \mathrm{~nm}$ reflectance increases as dust accumulates.

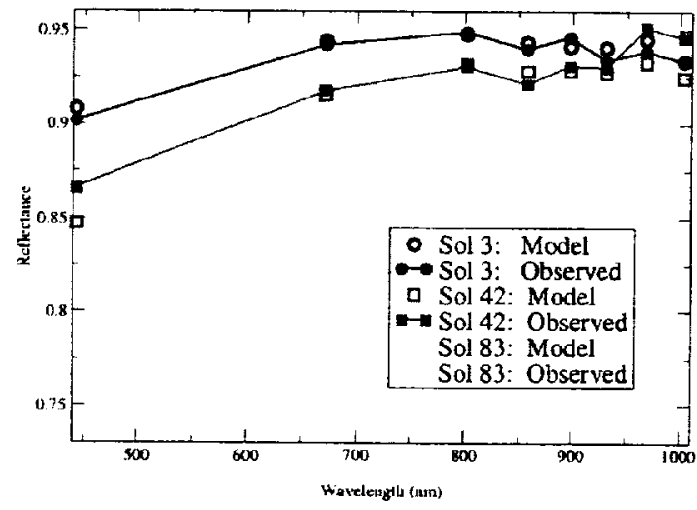

Figure 2. Observed and modeled left-eye spectra of RCT white ring for Sols 3, 42, and 83. Model assumed linear dust accumulation rate and isotropic phase function for dust having diameter $\mathrm{D}=5 \mu \mathrm{m}$.

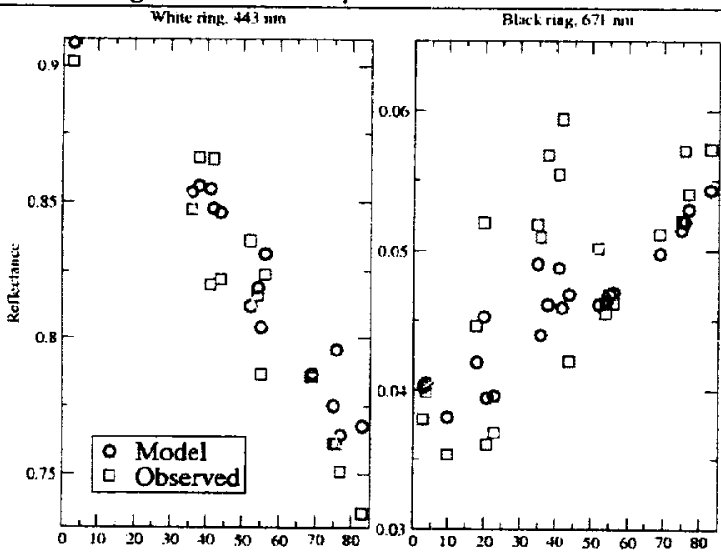

Figure 3. Observed and modeled reflectance as a function of Sol for white ring (at $443 \mathrm{~nm}$ ) and black ring (at 671 nm). Same model parameters used as in Figure 2. Green lines are linear fits to the observed values.

Slight variations between the observations and linear dust accumulation rate models suggested that models in which dust thickness (tau) was allowed to vary on a daily basis were warranted. Figure 4 demonstrates the results from one such model, in which 20 $\mu \mathrm{m}$ particles and an isotropic phase function were used to model the dust. The total dust accumulation of $\sim 0.1$ tau (corresponding to $\sim 6 \mu \mathrm{m}$ in this model) over 83 Sols is consistent with previous estimates of $20-25 \mu \mathrm{m}$ per year dust accumulation based on atmospheric fallout rates after dust storms, laboratory dust-coating experiments, and observations of dust obscuration at the
MPF site [10-12]. Daily variations in tau may be real or the effect of noise in the observational data, the sky model, and/or the two-layer model. We are currently studying how these different error sources may vary among the RCT surface types (white, gray, black) and how those uncertainties influence model results.

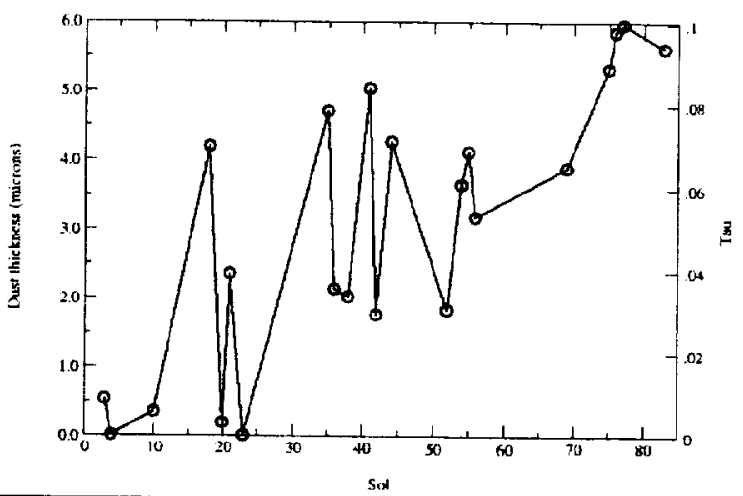

Figure 4. Computed dust optical depth tau variations (and corresponding coating thickness for assumed porosity 0.80 and grain size $D=20 \mu \mathrm{m}$ ) as a function of Sol using a model with an isotropic phase function and with a variable dust accumulation rate.

Conclusions. Radiometrically corrected IMP images of the Mars Pathfinder RCTs can be used to track the amount of deposited (or eroded) atmospheric dust at the MPF site. Two-layer radiative transfer models provide constraints on the dust opacity as it relates to grain size, porosity, and dust thickness, and may help constrain the spectral properties of the deposited dust.

Future work will incorporate new laboratory photometric measurements of palagonite-coated surfaces (both rocks and spare RCT materials to be used with MER Pancam instrument). More temporally- and radiometrically-controlled observations of the MER RCT target are planned for that mission.

References: [1] Reid, R.J., et al., JGR, 104, 8907, 1999; [2] Lemmon, M. et al., LPSC XXXII, \#2047, 2001; [3] Lemmon, M. et al., LPSC XXXIII, \#XXXX 2002; [4] Tomasko, M.G., et al., JGR, 104, 8987-9008, 1999; [5] Thomas, N. et al., JGR, 104, 8795-8808, 1999; [6] Johnson, J.R. and W.M. Grundy, GRL, 28, 2101-2104, 2001; [7] Johnson, J.R., M. Lemmon, W. Grundy, and K. Herkenhoff, LPSC XXXIII, \#1392, 2002; [8] Hapke, B., Theory of Reflectance and Emittance Spectroscopy, Cambridge Univ. Press, 455 pp., 1993; [9] Clark, R.N, et al., JGR, 95, 14643-14480, 1990; [10] Christensen, P., JGR, 91, 3533, 1986; [11] Landis, G., and P. Jenkins, $J G R, 105,1855,2000$; [12] Wells, E., et al., Icarus, 58, 331, 1984.

Acknowledgments. This work was funded by MDAP contract W-19, 898. Thanks to Bob Reid and Ken Herkenhoff, and of course Bruce Hapke for providing the tools necessary to investigate this problem. 
MICROSTRUCTURAL STUDIES OF SPACE WEATHERING EFFECTS IN LUNAR MATERIALS. L. P. Keller, Mail Code SR, NASA Johnson Space Center, Houston, TX 77058 (Lindsay.P.Keller@jsc.nasa.gov).

Introduction: Space weathering is a term used to include all of the processes which act on material exposed at the surface of a planetary or small body. In the case of the moon, it includes a variety of processes which have formed the lunar regolith, caused the maturation of lunar soils, and formed patina on rock surfaces. The processes include micrometeorite impact and reworking, implantation of solar wind and flare particles, radiation damage and chemical effects from solar particles and cosmic rays, interactions with the lunar atmosphere, and sputtering erosion and deposition. Space weathering effects collectively result in a reddened continuum slope, lowered albedo, and attenuated absorption features in reflectance spectra of lunar soils as compared to finely comminuted rocks from the same Apollo sites [1,2]. However, the regolith processes that cause these iffects are not well known, nor is the petrographic setting of the products of these processes fully understood.

Although it was initially proposed that impact vitrification was the main darkening agent acting in the lunar regolith, Hapke and co-workers showed that this process had only a minimal effect on regolith optical properties $[3,4]$. In a very important set of experiments, Hapke et al. showed that this coatings produced by vapor deposition of thermally evaporated analogs, and by the deposition of vapors generated by solar wind sputtering have optical properties that resemble those of the lunar fines [5]. In both of these depositional environments, the postulated darkening agent was submicroscopic Fe metal grains which are strong absorbers of visible wavelengths [5]. Although there existed extensive theoretical data to support an abundance of vapor-deposited material in the lunar regolith, the concept was resisted because of a lack of direct evidence of this material in lunar soils.

An interesting confluence of events occurred in the early 1990's. First, came the discovery of vapor deposited coatings on lunar regolith grains by Keller and McKay [6], who showed that amorphous coatings from $50-100 \mathrm{~nm}$ thick containing fine-grained Fe metal (1-10 $\mathrm{nm}$ in dia.) were common in the fine size fraction of several mature lunar soils. The other discovery was the recognition that the optical properties of lunar soils were dominated by fine grain sized material $(<45$ um fraction) by Pieters and coworkers [7]. These discoveries led to coordinated studies that looked at the mineralogy, chemistry, and optical properties of lunar soils as function of composition, maturity, and grain size fraction $[8,9]$. One of the major revelations from these studies was the recognition that much of the nanophase Fe metal is surface-correlated especially in the finest size fractions, and that it was this nanophase $F e$ that dominated the optical properties of the soil.

What we know: Nanophase $\mathrm{Fe}^{\circ}$ has been strongly implicated as one of the main agents in the modification of optical properties of lunar soils, associated with space weathering Recent work has shown that it the size, abundance, and petrographic setting of the nanophase $\mathrm{Fe}^{\circ}$ that determines the magnitude of the optical effects $[10,11]$. Nanophase $\mathrm{Fe}^{\mathrm{O}}$ occurs in two main petrographic settings in fine grained lunar soils: 1) as inclusions within agglutinitic glass produced by micrometeorite impact, and 2) as inclusions in thin amorphous rims surrounding soil grains that are formed by vapor deposition and irradiation effects. The average size of the nanophase $\mathrm{Fe}^{\mathrm{o}}$ in agglutinitic glass is a factor of $\sim 2$ larger than that observed in the amorphous rims. The average size of the nanophase $\mathrm{Fe}^{\circ}$, regardless of whether it is surface- or volume-correlated, appears to have a major effect on the optical properties of the host grain. The occurrence of $\mathrm{Fe}^{\circ}$ inclusions that are $>10 \mathrm{~nm}$ in diameter results in strong darkening in the corresponding reflectance spectra, whereas it is the smaller $\mathrm{Fe}^{\circ}$ grains $<5 \mathrm{~nm}$ diameter that give rise to strongly reddened slopes in the spectra [12]. It has been shown that in the fine size fraction of mature soils, nearly every grain has nanophase Fe metal within $100 \mathrm{~nm}$ of the grain surface [13]. The same situation exists for patinas on lunar rocks, which also contain abundant nanophase $\mathrm{Fe}$ metal on exposed surfaces [14].

All of the soil particles that we have analyzed to date have some accretionary material (e.g. splash glass, vapor deposits, sputter deposits, etc.) on their surfaces, although the amount of material can be highly variable. A common characteristic of the accretionary material is the occurrence of nanophase metal as randomly oriented inclusions or in layers. In the coated particles, the metal grains are concentrated in thin (50-150 nm thick) rims surrounding mineral grains (mainly plagioclase and augite). The large compositional difference between rims and their hosts led [6] to propose that the inclusion-rich rims on mineral grains likely result from depositional processes in the lunar regolith, either condensation of impact-generated vapors or sputter deposition. This accretionary material can have 
sition. This accretionary material can have profound effects on the optical properties of the soil grains.

Radiation effects can also produce surfacecorrelated nanophase Fe metal in Fe-bearing minerals such as ilmenite and olivine. Transmission electron microscope (TEM) analysis of the ilmenites shows that the grains are surrounded by altered rims up to $200 \mathrm{~nm}$ thick where $\mathrm{Fe}$ has been preferentially removed from the ilmenite surface, nanophase Fe metal grains have been produced, and the $\mathrm{Ti}$ has been partly reduced to $\mathrm{Ti}^{3+}$. While these altered rims contain submicroscopic metal grains, they do not have a large effect on the reflectance data. A more dramatic effect is observed in olivine grains. We have observed altered rims on olivine grains where much of the Fe within $\sim 50 \mathrm{~nm}$ of the surface has been reduced to nanophase Fe metal surrounded by $\mathrm{Mg}$-silicate glass. Spectra from the "altered" olivine grains are much darker than pristine olivine of similar composition.

Recent work has demonstrated that these space weathering effects (amorphous rims with nanophase $\mathrm{Fe}$ metal) can survive lithification, compaction and brecciation, at least for some lunar breccias [15].

What we do not know: An extensive observational database now exists on the space weathering effects in the lunar regolith in terms of changes in optical properties, surface microstructure, and chemistry. What is sorely lacking is an experimental database that will allow for quantification of the observational effects in terms of the processes involved. For example, there is still no consensus on the degree to which sputter deposition and vapor deposition (of material vaporized by micrometeorite impacts) have contributed to the deposited material observed on lunar soil grains. Similarly, it is clear from recent debates in the literature that certain minerals in the lunar regolith (ilmenite for example) behave differently than others (most silicates). Even within silicates, there is the open question of how Fe-bearing silicates undergo space weathering as compared to Fe-poor or Fe-free silicates. Finally, it is generally recognized now that the nanophase and submicroscopic Fe metal beads in melt glass, and in grain rims are the primary cause of the optical effects of space weathering. However, little is known about the specific processes involved - Is a hydrogen saturated surface required? Does sputtering produce significant nanophase Fe? What are the kinetics of these processes, i.e. how long does it take to form a vapordeposited rim $50 \mathrm{~nm}$ thick? Can the experience we have gained from studying space weathering effects in the lunar regolith be extrapolated to understanding space weathering in asteroidal regoliths [e.g. 16]?
References: [1] Adams J. B. and McCord T. B. (1970) GCA, Supp. 1, 1937, [2] Adams J. B. and McCord T. B. (1971) Science, 171, 567. [3] Hapke, B. et al. (1973) Trans. AGU, 54, 356. [4] Wells, E. and Hapke, B. (1977) Science, 195, 977. [5] Cassidy, W. and Hapke, B. (1975) Icarus, 25, 371. [6] Keller, L. P. and McKay, D. S. (1993) Science, 261, 1305. [7] Pieters, C. et al. (1993) JGR, 98, 20,817. [8] Taylor, L. A. et al. (2001) JGR, 106, 27,895. [9] Pieters, C. M. et al. (2000) MPS, 35, 1101. [10] Keller, L. P. and S. J. Clemett, S. J. (2001) LPS XXXII, Abstract \#2097. [11] Allen, C. C. et al. (1996) LPS XXVII, 13. [12] Keller, L. P. et al. (1998) New Views of the Moon, LPI Wkshp, 41. [13] Keller, L. P. et al. (2000) LPS XXXI, Abstr. \#1655. [14] Wentworth, S. et al. (1999) MPS, 34, 593. [15] Noble, S. K., et al. (2002) LPS XXXIII, \#1334. [16] Noble' S. K. et al. (2002) MPS, in press.

Acknowledgments: It is an honor and priviledge to be invited to this Symposium in honor of Dr. Bruce Hapke. This work was supported in part by NASA RTOP 344-31-40-07 (LPK).

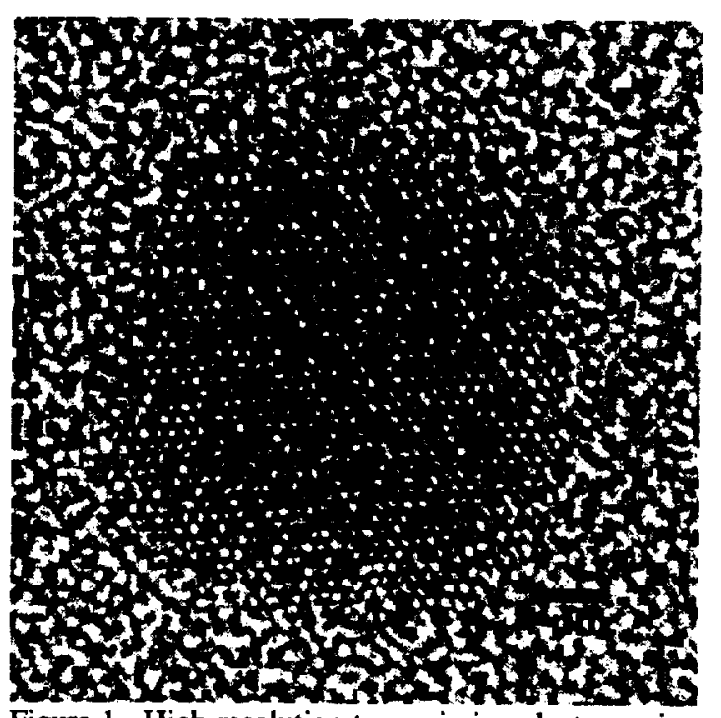

Figure 1. High-resolution transmission electron microscope image of a nanophase ( $7 \mathrm{~nm}$ dia.) Fe metal grain embedded in agglutinitic glass from 79221. 
Four Station Interferometric Kadar Observations of Mars. K.W. Larsen', A.F. Haldemann', R.F. Jurgens ${ }^{2}$, R.E. Arvidson', M.A. Slade ${ }^{2}$ ' Dept. of Earth and Planetary Sciences, Washington University, 1 Brookings Drive, St. Louis, MO 63130. Larsen@wunder.wustl.edu. ${ }^{2} \mathrm{JPL}$, California Institute of Technology, 4800 Oak Grove Dr. Pasadena, CA.

Introduction: Planetary targuts have been observed with radar since the late 1')50's when it was first used for ranging experiments with the Moon [1] As telescope size and power increased, it became possible to observe more distance taryets (Venus, Mars, and the outer satellites). Inherent to radar observations is the uncertainty as to the source of the reflection, there being two points where range and Doppler rings intersect on a sphere. The use of interferometric methods, first used on the moon with two stations [2] and later on Venus and Mars [3], solved this problem. We extend the method through the addition of a fourth receiving telescope (thus doubling the number of projected baselines) and integration of the newly available Mars Orbiter Laser Altimeter (MOLA) topographic datasets.

Observations, 2001: During the two and a half months surrounding the 2001 Mars opposition, fourteen nights of observations were conducted using four telescopes of the Goldstone Deep Space Communication Complex (GSDCC). The observations sampled a significant swath of the Martian equatorial region, coverage dependant on planetary geometry, sampling a diverse collection of geologic settings and conditions. For the purpose of developing and testing the four station interferometric technique, we have focused our efforts on a single night's data, that of July 14, 2001. This night's data has the advantages of a high signal to noise ratio on all four telescopes and a strong return from the moderately rough surface of Chryse Planitia. The extent of coverage on the Maltian surface is dependant on the geometry of the larth-Mars system, which determines the longitude range, and the distance to the first null phase point, covering approximately three degrees of latitude north and suuth.

Method: There are two phase: to the processing of the data. The first phase prepares the data for further processing by calibrating the raw dita stream and converting it from the time domain to range/delay-doppler space. The details of this phase are well known and covered in more detail elsewhere [4]. Figure $l$ is an example of the data at this stage of the processing and shows the relative signal to noise ratios between the four stations. We are primarily intcrested in the second phase of the processing, the interferometric method and modeling of the reflectiun coefficient.

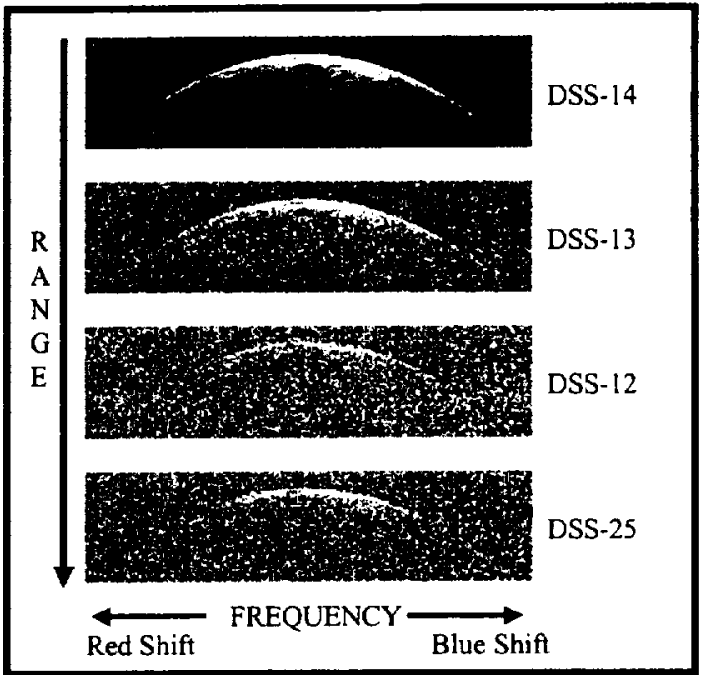

Figure 1: A typical set of range/delay-doppler images for July 14, 2001, approximately $10^{\circ}$ East Iongitude and $7^{\circ}$ North latitude. The frames have been similarly stretched to highlight the differences in the signal to noise between the four stations.

Past planetary interferometric radar experiments have successfully used data from two or three stations to map the surface reflectivity (or radar backscatter coefficient) [3]. The addition of a forth receiving telescope doubles the number of projected interferometric baselines. The equations for the observed signals (voltage) at each of the four telescopes at each point on the surface of Mars are as follows:

$$
\begin{aligned}
& V_{1}=V_{N}+V_{S}+n_{1} \\
& V_{2}=V_{N} e^{i u h_{n}}+V_{S} e^{i \omega h_{s}}+n_{2} \\
& V_{3}=V_{N} e^{i \nu h_{n}}+V_{S} e^{i \omega h_{s}}+n_{3} \\
& V_{4}=V_{N} e^{i w h_{n}}+V_{S} e^{i w h_{4}}+n_{4}
\end{aligned}
$$

Where $V_{x}$ is the complex voltage measured at each telescope, $n_{x}$ is a random gaussian noise, $V_{N, S}$ is the backscatter coefficient due to the northern and southern points respectively, $u, v$, and $w$ are spatial fringe frequencies due to the geometry of the stations and planets, and $h_{N, S}$ are the local heights of the northern and southern hemispheres. 


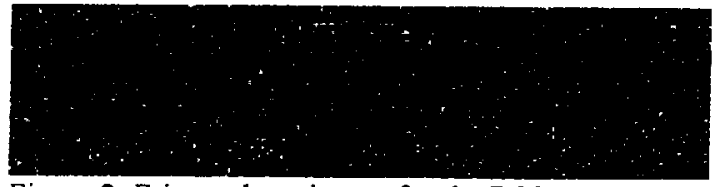

Figure 2: Fringe phase image for the DSS14-13 station pair. The cross-power image is colored such that the pixel brightness represents the power and the color the phase. Only the leading few pixels are easily viewable due to the predominance of the signal contained in the leading edge.

Since there is an independent gaussian noise signal inherent to every measurement, no single solution exists and the statistical solution is necessary. The iterative maximum likelihood function method (MLFM) has been used in the past and found to give the best results [5]. Equations 1-4 are represented such that one station is chosen as a phase-normalized reference station (Equation 1), the phases are assumed to be zero. The phases at the remaining three stations are then established to be relative to that of the first.

Furthermore, since the measured complex voltages at each station are zero-mean Gaussian random variables, as they are composed of the reflection from a large number of randomly oriented subresolution facets within each delay-doppler cell, the square of their magnitude is proportional to the backscattered power, $P_{\mathrm{N}, \mathrm{S}}$. The formulation of the maximum likelihood estimator is as follows.

$$
\left[\prod_{1}^{M} P(v)\right]^{1 / M}=\frac{K e^{-\frac{1}{m} \sum_{1}^{M}|u|^{2}} e^{\frac{B_{m} E+A_{m} f+C_{m}}{E f-D}}}{R_{N} R_{S}(E f-D)}
$$

Where there are $M$ stations, $P(v)$ is the crosspower spectra between two stations and has a northern and southern component. $K, A_{m}, B_{m}, C_{m}, E, f$, and $D$ are from variable changes that, for clarity's sake, are combinations of the power and cross-powers. Finally, $R_{N}$ and $R_{S}$ are the northern and southern reflectivities, for which we want to solve. By differentiating with respect to $P_{N}$ and $P_{S}$, setting the results equal to zero, and solving for $R_{N}$ and $R_{S}$ we arrive at the following coupled equations.

$$
\begin{aligned}
& R_{N}=\frac{A_{m} f^{2}+B_{m} D+C_{m} F}{(E f-D)\left(\frac{f}{\sigma^{2}}-D\right)} \\
& R_{S}=\frac{B_{m} E^{2}+C_{m} E+A_{m} D}{(E f-D)\left(\frac{E}{\sigma^{2}}-D\right)}
\end{aligned}
$$

Here, the variables are the same as for equation (5) with the addition of $\sigma^{2}$ which is the noise component of the signal. Since the terms on the right all contain $R_{N}$ and $R_{S}$, the coupled equations must be interated to a stable solution.

Unlike past experiments where, in addition to iterating the maximum likelihood solution for the reflectivities, the altitude of the reflecting pixels also an unknown, we now possess an extremely accurate topographic map courtesy of MOLA. We use the MOLA topography as an input into the solution, thus eliminating the two height variables and simplifying the calculation.

The actual application of the MOLA topography in the radar solution is complicated by both the size of the resolution cell (the observed reflected power is due to reflection from a large number of small facets) and the antenna response function [6]. Thus, the target response function (a combination of the altitude and orientation of facets within a resolution cell) is convolved with the antenna ambiguity function to properly weight the altitude.

Finally, with the completion of the MLFM processing, we have 2-d maps of the surface reflectivity across the observation regions. We model the reflectivity using Hagfors' model, where $R$ is the reflectivity, $C^{-1 / 2}$ is the rms slope, $\rho$ is the fresnel reflectivity, and $\theta$ is the incidence angle.

$R=\frac{C \rho}{2}\left(\cos ^{4} \theta+C \sin ^{2} \theta\right)^{-3 / 2}$

References: [1] Hagfors T. (1961) JGR, 66, 777785. [2] Shapiro, I.I. et al. (1972) Science, 178, 939948. [3] Jurgens, R.F. et al. (1980) JGR, 85, 82828294. [4] Larsen, K.W. et al. (2002) LPSC XXXIII. [5] Rumsey, H.C. et al. (1974), Icarus, 23, 1-7. [6] Evans, J.V. and Hagfors, T. (1968) Radar Astronomy, McGraw-Hill Book Company. 
A COMPLETE FIRST ORDLR HAPKE MODEL OF THE NEAR-INFRARED SPECTRAL REFLECTANCE OF THE MOON. P. G. Lucey, Hawaii Institute of Geophysics and Planetology, University of Hawaii at Manoa, 2525 Correa Road. I Ionolulu HI 96822 (lucey@higp.hawaii.edu).

Introduction: The spectral reflectance properties of the Moon are governed by the minerals and glasses composing the lunar regolith, their physical state, and the optical effects of soil maturity. Work by Hapke and others has provided all the tools necessary to produce a model of near-IR spectra of the Moon within simplifying assumptions. We have produced such a model and are beginning to apply i: to lunar science problems. Two classes of problems are amenable to immediate use: determining compssitions of lunar surface regions using groundbased and Clementine data, and understanding the detection limits for minerals and rock types using existing and planned data sets.

Model: Our model is based on the equations of Hapke [1] who showed how the visible and near-IR spectra of mixtures of minerals could be computed from their optical constants at arbitrary grain sizes and relative abundances, and recently it $w$ as shown how the method of [1] could be modified to include the effects of submicroscopic particles [2].

Optical constants of all components are required for this model. An inversion of the Hapke equations was presented by [3] in order to compute optical constants from reflectance spectra. We used this inversion to compute the optical constants of anorthite from a spectrum of the mineral in the USGS Denver spectrum library. For the optical constants of olsvine we used the the optical constants computed by [3]. For pyroxene we applied a new methodology wheren we fit the optical constant spectra of a series of pyr 'xenes with Gaussians, then regressed the Gaussian parameters on composition, ignoring Gaussians we believed due to water or iron oxide contaminants. We simulated the spectrum of shocked anorthite by interpolating across the 1250 ferrous iron band of the ISGS plagioclase. For native iron, no measurements of these constants exist at the spectral sampling of lunat telescopic measurements, but from 600 to $2000 \mathrm{~nm}$ the measurements of [4] Johnson and Christy (1974) scatter randomly about straight lines for both $\mathrm{n}$ and $\mathrm{k}$, so we used linear fits to the Johnson and Christy dat. I to represent the optical constants of Fe.

In the forward implementation of the model, the chemistry of minerals, grain sizes, modal abundances and abundance of submicroscopic iron are defined. From the mineral and glass chemistries optical constants are computed, which are then modified by submicroscopic iron coatings. These modified optical constants are then converted to single: scattering albedo and mixed according to their modal abundances.
Results: Qualitatively, the model produces spectra which closely mimic the appearance of lunar spectra, sharing albedo, continuum slope, and spectral contrast, as well as the shape of the absorption features. Validation of the forward model is proceeding use the spectra and analyses of [5]. The validation will determine, for example, when the model prescribes a certain grain size, or grain size distribution, how this optical grain size corresponds to that measured via sieving.

An important unresolved measurement involves submicroscopic iron. Hapke showed that the optical effects of iron extend far beyond the range detected by ferromagnetic resonance. Work by Morris shows that the correlation between various small grain sizes of iron is relatively poor. Thus, measurements of $\mathrm{Is} / \mathrm{FeO}$ can only weakly constrain the amount of submicroscopic iron prescribed by our model to best fit lunar spectra. Future lunar soil validation measurements should measure a wider, or additional, iron size ranges.

While the validation dprocess is proceeding, we have applied the model to lunar science problems that do not depend critically on absolute abundances. For example, we recently showed that areas on the Moon previously interpreted to be composed of anorthosite that had its 1.25 micron band erased by shock, can plausibly be mimicked by spectra of mature crystalline anorthosite [6].

A recently introduced application of this model is to determine detection limits for minerals as a function of soil maturity and data quality (signal to noise ratio). In this process we model a particular soil composition, ensuring that the model spectrum lies within the field of measured lunar spectral properties. We then vary modal and chemical properties to determine the magnitude of the differential signal that can be detected by a remote sensor. Our preliminary results suggest that $10 \%$ differences in mineralogy can only be detected for the most immature surfaces at the $1 \%$ precision of Clementine and groundbased data. Future lunar missions should features sensors with much higher signal to noise ratios.

References: [1] Hapke, B., Theory of Reflectance and Emittance Spectroscopy, Cambridge Univ. Press, Cambridge, 1993, [2] Hapke, B., , J. Geophys. Res., 106, E5, 10,03910,074, 2001., [3] Lucey, P.G, J. Geophys. Res., 103, E1, pp1703-1714, 1998. [4] Johnson, P and R. Christy, Phys. Rev. B9, 5056-5070, 1975, [5] Taylor L. A., Pieters C. M. Keller L. P., Morris R. V., McKay D. S. (2001), J. Geophys. Res. 106, 27,985-27,999., [6] Lucey, P.G. GRL in press. 
. 
COHERENT BACKSCATTERING BY RANDOM PARTICULATE MEDIA IN THE SOLAR SYSTEM. K. Muinonen, Observatory, University of Helsinki, P. O. Box 14,FIN-00014 U. Helsinki, Finland (Karri.Muinonen@ Helsinki.Fi).

Photometric observations for larg: numbers of asteroids indicate an opposition effect, a nonlincar increase of brightness at small solar phase angles, the angle between the Sun and the observer as seen from the asteroid. Vast sets of asteroid polarimetric observations show negative polarization, a peculiar degree of linear polarization $\left(I_{\perp}-I_{\|}\right) /\left(I_{\perp}+I_{\|}\right)$ for unpolarized incident sunlight: at imall phase angles, the brightness component $I_{i}$ with the elt tric vector parallel to the scattering plane defined by the Sun, the asteroid, and the observes predominates over the perpendicular component $I_{\perp}$. For recent reviews, see Muinonen et al (2002ab).

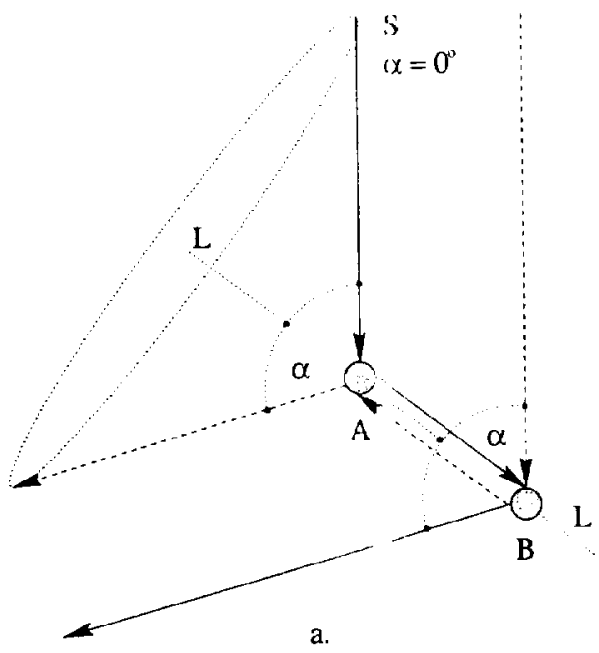

The coherent backscattering me hanism (CBM) for the opposition effect is described in Fig. $t$ a for second-order scattering. An incident electromagnetic plane wave (solid and dashed lines; wavelength $\lambda$ and wavenumber $k=2 \pi / \lambda)$ interacts with two scatterers $A$ and $B$, which are of the order of the wavelength to hundreds of wavtlengths apart, and propagates to the observer to the left. The two scattered wave components due to the two opposite propagation directions between the scatterers interfere constructively in the conical directions defined by rotating the light source direction $S$ about the axis $L$ joining the two end scattertrs. We illustrate a scattering direction on the cone precisely opposite to the light source direction. Thus, the exact backward direction (phase angle $\alpha=0^{\circ}$ ) is on the constructive-interference cone for arbitrary locations of the two scatterers whereas, in other directions, interference varies from constructive to destructive. Three-dimensional averaging over scatterer locations results in a backscattering enhancement with jecreasing angular width for increasing order of interactions, hecause the average distance between the end scatterers is larger for higher orders of interactions. The scattering processes can be caused by any disorder or inegularity in the medium.

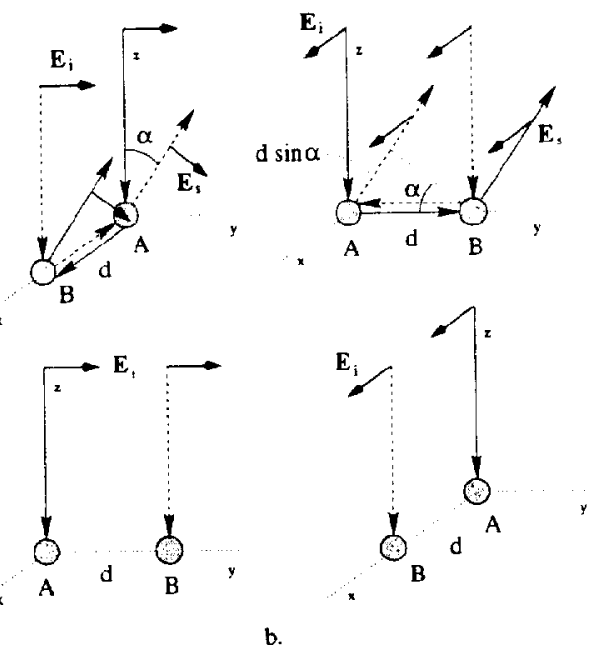

Figure 1: Coherent backscattering mechanism for (a) the opposition effect and (b) the negative polarization. See text.

CBM for the negative degree of linear polarization is explained for second-order scattering in Fig. Ib. The incident radiation is unpolarized by definition, which requires the derivation and proper averaging of the Stokes vectors corresponding to the scattered electromagnetic fields $\left(\boldsymbol{E}_{s}\right)$ for two linear polarization states of an incident plane wave $\left(\boldsymbol{E}_{\boldsymbol{i}}\right)$. In Fig. Ib, incident polarizations parallel and perpendicular to the scattering plane (here $y z$-plane) are treated in the two leftmost and two rightmost panels, respectively.

Consequently, in Fig. 1b, an incident electromagnetic plane wave interacts with two scatterers $A$ and $B$ at a distance $d$ from one another aligned either on the $x$-axis or the $y$-axis. while the observer is in the $y z$-plane. For the present geometries, the constructive interference cones of Fig. la reduce to the $y z$ and $x z$-planes, depending on the alignment of the scatterers. Since first-order scattering is typically positively polarized (e.g., Rayleigh scattering and Fresnel reflection), the scatterers sufficiently far away from each other $(k d=2 \pi d / \lambda \gg 1)$ interact predominantly with the electric field vector perpendicular to the plane defined by the source and the scatterers (two upper panels), while interaction with the electric field vector parallel to that plane is suppressed (two lower panels). The observer in the $y z$-plane will detect negative polarization from the geometry in the upper left panel of Fig. 1, and positive polarization from the geometry in the upper right panel. However, the positive polarization suffers from the phase difference $k d \sin \alpha$, whereas the phase difference for the negative polarization is zero for all phase angles. Averaging over scatterer 


\section{COHERENT BACKSCATTERING: K. Muinonen}

locations will result in negative polarization near the backward direction. Scattering orders higher than the second experience similar preferential interaction geometries, and contribute to negative polarization. As above for the opposition effect, the contributions from increasing orders of scattering manifest themselves at decreasing phase angles.

Figure 2 shows tentative modeling of the opposition effect and negative polarization of $\mathrm{C}, \mathrm{M}, \mathrm{S}, \mathrm{V}$, and E-class asteroids using Monte Carlo computations for coherent backscattering (Muinonen 2002; Muinonen et al. 2002ab). The novel numerical technique relies on the reciprocity principle for electromagnetic scattering, allowing a renormalization of the coherent backscattering contributions at each multiple-scattering event.

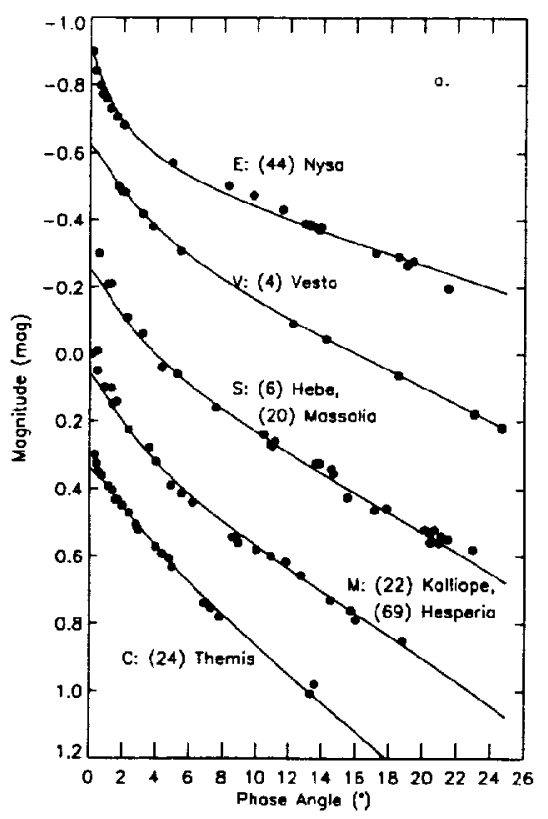

Coherent backscattering is here computed for a plane wave (wavelength $\lambda=2 \pi / 10 \mu \mathrm{m}$ ) normally incident on a semjinfinite medium of discrete Rayleigh scatterers with singlescattering albedos of 0.9 and medium mean-free-path length of $\ell=1,2,3, \ldots, 50 \mu \mathrm{m}$. For Rayleigh scatterers, the singlescattering albedo chosen roughly results in a maximum opposition effect amplitude as well as a maximum depth of the negative polarization surge. $5 \times 10^{4}$ rays were used in each computation

In Fig. 2, an ad hoc exponential weighting scheme of $\exp (-\ell / L)$ has been applied to the numerical results in all cases with $L=1.0,2.0,2.0,2.0$, and $6.0 \mu \mathrm{m}$ for the $\mathrm{C}, \mathrm{M}$, $\mathrm{S}, \mathrm{V}$, and E-class curves, respectively. The weighting scheme derives from the idea that, rather than modeling the random scattering medium using a single mean free path only, we allow a distribution of mean free paths and thus physical characteristics on asteroid surfaces. Additionally, the CBM computations have been multiplied by a linear function $1-K \alpha$, where $K=0.021,0.017,0.015,0.013$, and $0.009 \rho$ for C, M, S, V and E-class curves, respectively. For comparison with the polarimetric observations, the negative polarization surges have been divided by the scaling "fudge factors" of $1.5,2.3,3.0$ 4.0 , and 7.2. The numerous implications of the tentative fits are subject to future discussion.

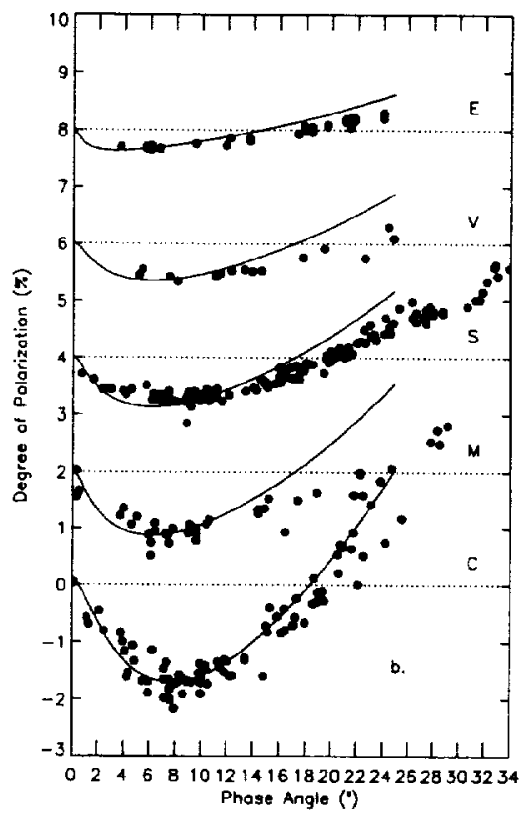

Figure 2: Opposition effect (a) and negative linear polarization observations (b) for C, M, S, V, and E-class asteroids with heuristic modeling. For the references of the observations, see Muinonen et al. (2002a)

K. Muinonen (2002). Coherent backscattering by absorbing and scattering media. In Electromagnetic and Light Scattering by Non-spherical Particles, Gainesville 2002 (B. Gustafson, L. Kolokolova, and G. Videen, eds., Army Research Laboratory, Adelphi, Maryland, U.S.A.), 223-226.

K. Muinonen, J. Piironen, Yu. G. Shkuratov, A. Ovcharenko, and B. E. Clark (2002a). Asteroid photometric and polarimetric phase effects. In Asteroids III (W. Bottke, R. P. Binzel, A. Cellino, P. Paolicchi, Eds., University of Arizona Press, Tucson, Arizona, U.S.A.), in press.

K. Muinonen, G. Videen, E. Zubko, and Yu. Shkuratov, Yu. (2002b). Numerical techniques for backscattering by random media. In Cosmic Dust and Its Optics: Bratislava Contributions (G. Videen and M. Kocifaj, eds., Kluwer Academic Publishers, Dordrecht), in press. 
CRISM: COMPACT RECONNAISSANCE IMAGING SPECTROMETER FOR MARS ON THE MARS RECONNAISSANCE ORBITER. S. Murchie ${ }^{1}$, R. Arvidson ${ }^{2}$, O. Barnouin-Jha ${ }^{3}, \mathrm{~K}$. Beisser ${ }^{1}, \mathrm{~J}$.-P. Bibring ${ }^{3}, \mathrm{~J}$. Bishop $^{4}$, J. Boldt ${ }^{3}$, T. Choo ${ }^{3}$, R.T. (lancy ${ }^{5}$, E.H. Darlington ${ }^{3}$, D. Des Marais ${ }^{4}$, D. Fort', R. Green ${ }^{33}$, J. Hayes ${ }^{3}$, J. Lees $^{1}$, E. Malaret ${ }^{6}$, D. Mehoke ${ }^{3}$, R. Morris ${ }^{7}$, J. Mustard ${ }^{8}$, K. Peacock ${ }^{3}$, M. Robinson', T. Roush ${ }^{4}$, E. Schaefer ${ }^{3}$, P. Silverglate ${ }^{1}$, M. Smith ${ }^{10}$, P. Thom 'son $^{3}$, and B. Tossman', ${ }^{1}$ Applied Physics Laboratory, Laurel, MD 20723, ${ }^{2}$ Washington University, St. Louis, MO, ${ }^{3}$ Institut d'Astrophysique Spatiale, Orsay, France, ${ }^{4}$ NASA ARC, Moffett Field, CA, ${ }^{5}$ Space Science Institute. Boulder, CO. ${ }^{6}$ Applied Coherent Technology, Herndon, VA, ${ }^{7}$ NASA/JSC, Houston, TX, ${ }^{8}$ Brown University, Providence, RI, ${ }^{9}$ Northwestern University, Evanston, IL, ${ }^{10}$ NASA/GSFC, Greenbelt, MD, ${ }^{33}$ NASA/JPL, Pasadena, $C A$.

Introduction: The Compact Recunnaissance Imaging Spectrometer for Mars (CRISM) on the Mars Reconnaissance Orbiter (MRO) will cinduct a comprehensive series of investigations of the Martian surface and atmosphere. The investigations will be accomplished using an instrument design that provides high spatial and spectral resolutions, extended wavelength range, and ability to gimbal through ، range of orientations. Baseline investigations include a near-global survey to find high science priority sites, full-resolution measurement of thousands of such sites, and tracking of seasonal variations in atmospheric and surface properties.

Science Overview: The Mars Exploration Payload Analysis Group [1] recommended specific hyperspectral imaging investigations to characterize Martian geology, climate, and environments of present or past life. CRISM's three groups of investigations address all of MEPAG's recommendations. The first two groups correspond to two primary objectives of MRO: to search for evidence of aqueous and/or hydrothermal activity, and to map and characterize the composition, geology, and stratigraphy of surface features. These investigations are implemented by high-resolution hyperspectral mapping of thousands of high priority targets including candidate sedimentary deposits [2], volcanic regions, crustal sections exposed in steep escarpments, and sites which exhibit evidence in Mars Express/Omega data for concentrations of aqueously formed minerals. The third group of investigations addresses the primary MRO objective to characterize seasonal variations in dust and ice aerosols and water content of surface materials, and the secondary objective to provide information on the atmosphere complementary to other MRO instruments. These investigations are implemented using a systematic, global grid of measurements of the emission phise function (EPF) acquired repetitively throughout the Martian year. EPF measurements allow accurate determination of column abundances of water vapor, $\mathrm{CO}$, dust and ice aerosols, and their seasonal variations [3]. At the same time, the grid's repetitive coverage will track seasonal variations in water content of surface material. Additional, tar- geted observations of the polar caps will investigate their inventory of water and $\mathrm{CO}_{2}$ ices.

When not taking targeted measurements, CRISM will conduct a $\sim 100 \mathrm{~m} / \mathrm{pixel}, \sim 59$-wavelength survey to search for evidence of aqueous activity that lacks morphologic expression and/or is below the resolution of previous spectral mapping. The survey addresses MRO's secondary objective to identify new sites with high science potential for future investigation, and will be particularly important for identification of key Noachian deposits. For example, even at low Martian erosion rates [4], morphologic expressions of Noachian hot spring deposits would have been removed in $\sim 10^{9}$ yrs, so that such deposits may now exist only as mineralized spots in morphologically unremarkable eroded escarpments, crater ejecta, and talus. Much of the survey will be completed before MRO's highest downlink rates, so that newly discovered sites can be targeted with full-resolution coverage.

Instrument Overview: An overview of the instrument is shown in Figure 1. CRISM's two major subassemblies are the Optical Sensor Unit (OSU) and the Data Processing Unit (DPU). The OSU contains visible (VIS) and infrared (IR) imaging spectrographs that share the same $2.05^{\circ}$ field-of-view and cover the wavelength ranges $400-1050 \mathrm{~nm}$ and $1050-4050$ at 7 $\mathrm{nm} /$ channel. The $\mathrm{R}$ focal plane is cooled with doubly redundant cryogenic coolers. A side-facing radiator cools the spectrometer cavity to decrease instrument background. An onboard integrating sphere provides radiance calibration, and a shutter and dedicated dark pixels on each focal plane provide background measurements interleaved with Mars measurements. Optics and the focal planes are enclosed in a housing that is gimballed by a high-precision motor/encoder $\pm 60^{\circ}$ along-track from nadir. During measurement of a target, along-track scanning takes out most groundtrack speed and allows long integration times and high signal-to-noise ratio (SNR) data to be obtained. At the same time, scanning provides the capability to remeasure a spot repeatedly at multiple emission angles to characterize the EPF. High spatial resolution $(-18$ $\mathrm{m} /$ pixel from $300 \mathrm{~km}$ altitude) allows characterization of the surface at the outcrop scale, and wavelength 
coverage to $>4000 \mathrm{~nm}$ provides sensitivity to carbonates even at low abundances. The DPU provides lossless or lossy compression of the data stream in real time. Key design elements are adapted from the CONTOUR and MESSENGER optical instruments.

Operations Overview: Figure 2 summarizes CRISM's three complementary data acquisition strategies, which are built around the three steps needed to characterize high scientific priority, small-scale deposits: find the deposits, separate their signature from that of the atmosphere, and acquire high spectral and spatial resolution measurements with high SNR. Most of the time the OSU views nadir, building up coverage in multispectral survey mode. In the second mode of observing, for a group of -36 orbits once every $\sim 30^{\circ}$ of Ls, EPFs are measured on a $10^{\circ}$ longitude $\times 15^{\circ}$ latitude grid. The third mode is targeted observations, approximately 2200 of which will be obtained at key locations. At each site, gimballing is used to take out most along-track motion, so that the field-of-view is slowly scanned over a rectangular swath approximately $11 \mathrm{~km}$ wide (when measured from $300 \mathrm{~km}$ altitude). Measurements of the swath are bracketed by incoming and outgoing measurements of the EPF of the centerpoint of the swath at emission angles up to $\pm 60^{\circ}$. Larger high-priority targets will be identified in Mars Express/Omega data and other data sets. The multispec- tral survey provides redundancy to Omega data in locating targets that have VIS-IR spectral signatures but lack obvious morphologic expressions, and it provides the spatial resolution needed to identify small targets not evident in Omega data.

References: [1] MEPAG, Mars Exploration Program: Scientific Goals, Objectives, and Priorities, 2000. [2] Cabrol, N. and E. Grin, Icarus, I42, 160 172, 1999. [3] Clancy, R.T., and S. Lee, Icarus, 93, 135-158, 1991. [4] Presley, M., and R. Arvidson, Icarus, 75, 499-517, 1988.

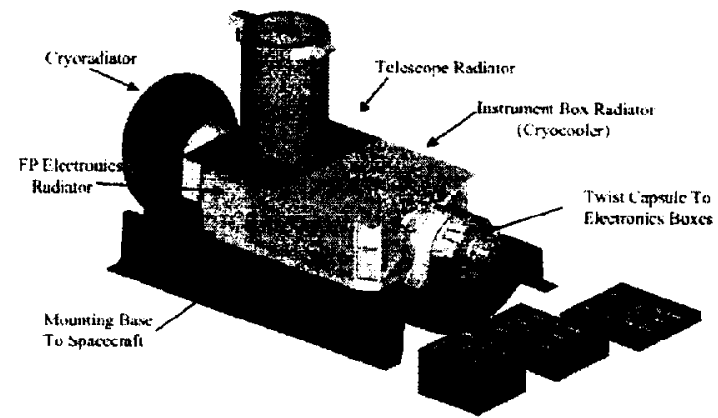

Fig. 1. Preliminary CAD renderings of CRISM's OSU, DPU, and control electronics.

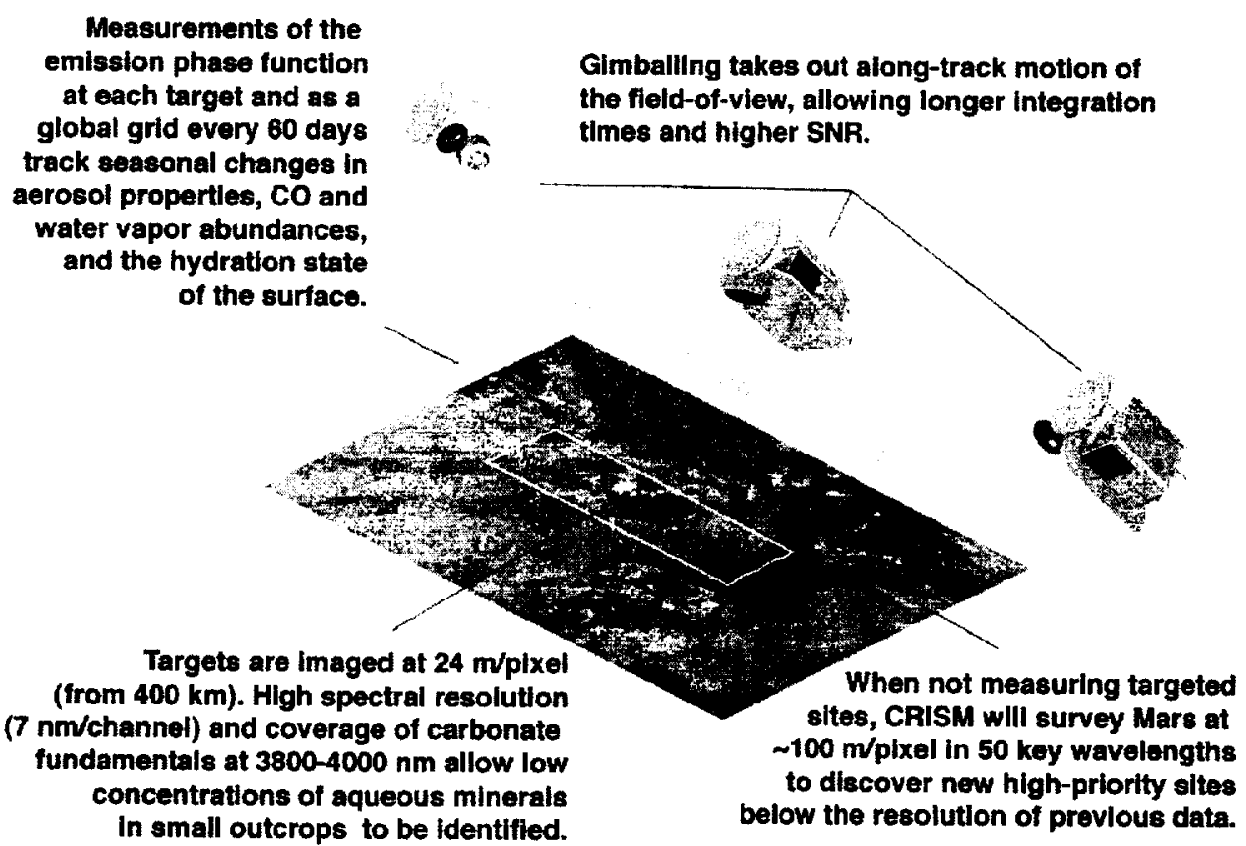

Fig. 2. Schematic depiction of key attributes of CRISM's measurement plan for the Martian surface and atmosphere. 
SMALL PARTICLES AND CEMENTED TEXTURES: TWO CHALLENGES FOR REMOTE COMPOSITIONAL ANALYSIS. J. F. Mustard', 'A Department of Geological Sciences, Box 1846, Brown University, Providence RI, 02912 John Mlustard@ibrown.edu.

Introduction: Hapke s [1] radiative transfer model for particulate surfaces has been demonstrated to be accurate for describing the reflectance of surfaces where the particles are larger than the wavelength of light (geometric optics regime). However, for particles that are smaller than the wavelength of light, the behavior of radiation in a particulate surface is different and not well described by geometric optics. Combining Mie and Hapke theories has been shown as a potentially promising avenue in modeling the reflectance of fine particle surfaces $[2,3]$ through these studies have also shown that important discrepancies between models and observations exist. Another important area of basic remote sensing research is $n$ the changes that occur in reflectance due to cementa1ion [4]. Processes that occur in soils can lead to cementation of particulates such as the formation of caliche or hardpans, or in the case of evaporite basins. Observations of the reflectance of particulate and cemented materials show that the change due to cementation are systematic and distinct from compaction or other effects, but have not been modeled [4]. Нere I describc experimental observations and modeling of these two cases (fine particles and cementation) and discuss possible paths to resolve these problems.

Fine Particle Reflectance: Mustard and Hays [3] measured the reflectance of particle size separates of 5 $\mathrm{m}$ intervals between 0 and $25 \mathrm{~m}$ of the minerals ol ivine and quartz to examine the spectral properties of these materials when the particle size is greater than, approximately the same size as, and then much smaller than the wavelength. They used the wavelength range 0.3-25 m which, when combined with the size separates used, allowed examination of this processes across a range of optical constant: expressed by the minerals. The expectation was that for a given particle size separate at some point, and likely multiple points, along this wavelength range the transition from optical to Mie scattering should occur.

The results of these measurements indeed demonstrated the expected effects. When the particle size approached, and then became smaller, than the wavelength of light, the reflectance exllibited a sharp decrease. This is predicted to some extent by the size parameter which relates the particle size to the wavelength of light. However, the size parameter needs to be combined with concepts of extinction efficiency to fully explain the observations. Mustard and Hays therefore defined a critical diameter, Dc (equation 1, ) $\mathrm{n}=$ real part of the complex index of refraction), based on Mie and scattering theory [1]. When Dc is less than 2 , then the reflectance should experience a sharp decrease. This relationship is only valid for optical re-

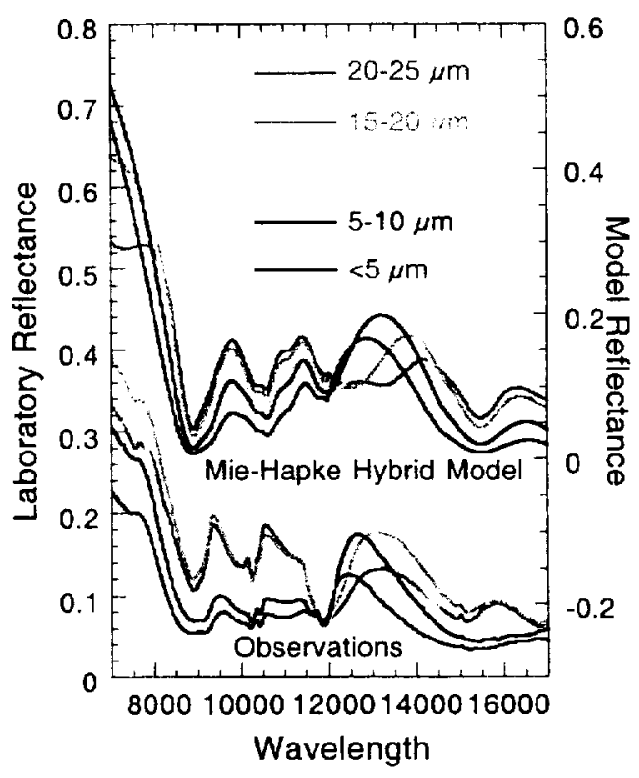

Figure 1. Comparison of measured and modeled reflectance spectra of fine particle separates of olivine

gimes where the imaginary component of the index of refraction is small. Mustard and Hays [3] demonstrated that the wavelengths where quartz and olivine should exhibit a sharp decline in reflectance on the basis of the particle size and the index of refraction corresponded well with observations.

$$
D c=\frac{2 \lambda}{\pi(n-1)}
$$

Modeling of these same observations had mixed results. A Mie model was used to calculate singlescattering albedo and a Hapke [1] model to determine reflectance, and inputs to the model were optical constants and particle size distribution. For olivine (Figure 1), the model agrees with observation in terms of trends: relative albedo of the separates for the various scattering regimes was correct, transitions and crossovers of reflectance occurred at approximately the same wavelengths. Although the model and observations differ in their details, this could be due to the optical constants used. For quartz, however, it was quite a different story. The model was consistent with the 
observations only in the most crude sense, and the details were significantly different. Here again, the suspicion is that with appropriate optical constants, perhaps a better agreement could be attained.

Cemented Soils: Cooper and Mustard [4] examined the effects of cementation on the reflectance spectra of Mars analogs. The samples consisted of mixtures of sulfate and JSC-Mars 1, a martian soil analog derived from Hawaiian palagonite, with proportions that matched the amount of sulfur detected by

\section{Loose Powders}

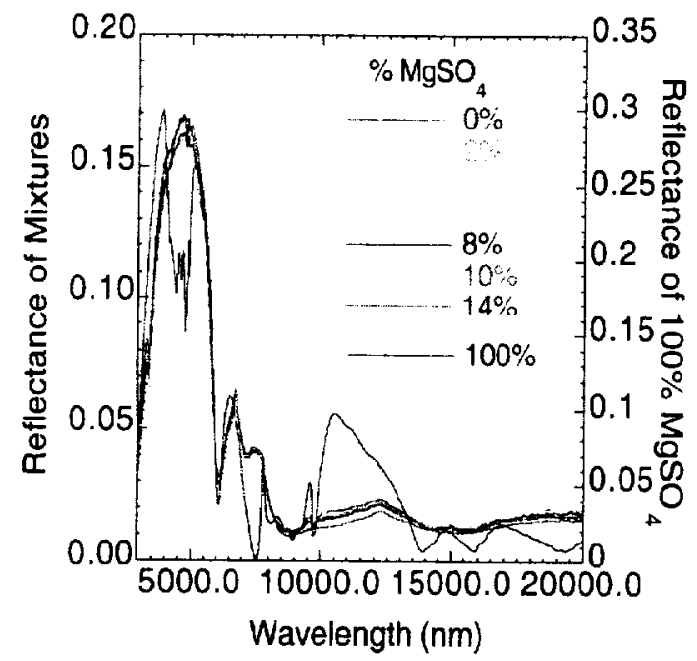

Figure 2a. Reflectance spectra of JSC Mars- 1 and $\mathrm{MgSO}_{4}$ mixtures. Note the vertical axis for $\mathrm{MgSO}_{4}$ is different.

the Viking and Pathfinder landers. The samples were measured as powders, packed powders, and cemented (details of these preparations are presented in [5]).

The spectra of the loose soils vs their cemented counterparts were largely unchanged in the visible to near infrared. However, in the thermal IR $(7-20 \mathrm{~m})$ significant changes did occur (Figure 2ab). The sulfate features around $9 \mathrm{~m}$ were barely identifiable in the powdered sample but became greatly enhanced in the cemented soils. This was understood from a physical perspective. The optical constants for sulfate become large at the $9 \mathrm{~m}$ features, creating a restrahlen band. When in the powdered form, the restrahlen band is weak due to multiple scattering. However, after cementation, the radiation sees a more coherent surface and multiple scattering is reduced creating an enhanced restrahlen feature compared to the iso-chemical powdered counterpart. It is interesting to note, however, that the JSC-Mars 1 sample also exhibits strong changes in the spectral properties in the thermal IR, though the cause of these differences were not explored by [5].

While these data showed drammatic changes that are readily understood, these effects have not yet been modeled. This could have much application for understanding Mars as the new generation of high spatial and spectral data sets become available. It appears that the combination of visible-NIR with thermal IR data could provide compositional and textural information if properly modeled.

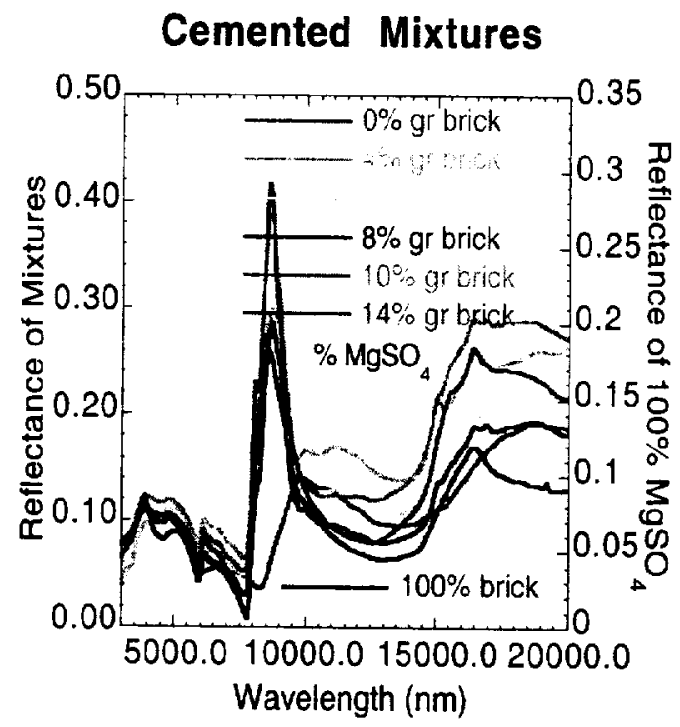

Figure 2b. Same samples as in 3 a but after cementation as described in text. Note change in vertical axis for mixtures relative to $2 \mathrm{a}$ and $\mathrm{b}$

Challenges: Laboratory observations of very fine particle separates indicate that theory provides a basis for understanding the observations, but there are significant problems in applying quantitative models. Similarly, theory provides a sound foundation for understanding the effects of cementation, but has not yet been applied or developed to treat this texture. Fine particles and cementation are two important textures expected on Mars and these effects will likely be present in new data sets to be collected. Thus it will be important to develop a better modeling base to analyze these problems.

References: [1] Hapke, B. 1993. Theory of Reflectance and Emittance Spectroscopy, Cambridge University Press, Cambridge. [2]'Moersch, J. E and P. R. Christensen (1995) J. Geophys. Res., 100, 7465-7477 [3]'Mustard, J. F. and J. E. Hays, (1997), Icarus, 125, 145-163. [4] Cooper, C. and J. F. Mustard (2002) Spectroscopy of loose and cemented sulfatebearing soils: Implications for duricrust on Mars, Icarus, (in press). 
THE OPPOSITION EFFECT: A VERY UNUSUAL CASE. R. M. Nelson, 183-501 Jet Propulsion Laboratory, 4800 Oak Grove Drive, Pasadena C\} 9 1 1 0 9 \text { , Robert.M.Nelson@ipl.nasa.gov. }

The reflection of electromagnetic radiation from a planetary regolith involves a combitation of geometric and physical optics processes which contribute to the signal returned to the remote obsencr. The geometric optics effects are the product of sangly and multiply scattered radiation from the surfaces of the regolith particles, combined with radiation which has undergone various combinations of transnussion through one or more regolith grains followed by one or more scatterings from other particles. The physical optics effects include diffraction of radiation around the cdges of large irregular particles and cosperative coherent scattering between particles which are small when compared to the wavelength of the incident radiation. These effects produce measurable changes in the intensity and polarization of reflected light as a function of illumination and viewing geometry. In particular, as phase angle becomes small, the reflectance of a particulate material will increase non-linearly and exhibit the 'opposition effect' [1]. In the planetary science context, the phase curve, and in particular the size of the opposition surge and the width of the phase curve near zero degrees, have been attributed to two processes commonly called 'shadow hiding' (SHOE) and 'coherent backscattering' (CBOE) [2,3,4] Understanding the contribution of the SHOE and CBOE components to the integrated phase curve will permit models to be developed which can confidently determine the fundamental regolith textural properties such as particle size and packing density from remote sensing data.

Laboratory experiments have attempted to distinguish the contribution of SHOE and CBOE by presenting samples with circularly polarized monochromatic electromagnetic radiation and measuring the circular polarization ratio in the retumed signal. If the returned signal is singly scattered then the circularization polarization ratio should decrease as phase angle decreases. However, if the returned signal is multiply scattered then the circular polarization ratio should strongly increase as phase angle decreases. We have observed this increase in circular polarization ratio with decreasing phase angle in many highly reflective particulate media $[5,6,7]$.

In the case of materials with lou reflectance the expectation is that most of the returisd signal is singly scattered because of the high probability of a photon being absorbed at each scattering. Hence, for absorbing media the circular polarization ratic is not expected to sharply increase as phase angle decreases. In general we have found this to be true [5]. However, recently we have encountered an interesting counter example. Measurements on a suite of boron carbide samples (reflectance $=\sim 5 \%$ ) have found a significant increase in circular polarization ratio with decreasing phase angle, a result that is not consistent with our interpretation of the process. This result suggests that albedo alone is not the principal regulator of the amount of multiple scattering in the medium. The explanation for this unusual behavior remains unknown; a conjecture might be that a unique particle shape may create a very unusual single scattering phase function in boron carbide.

It is important to understand this unusual behavior before we can proceed with development of models that can accurately retrieve textural properties from remote sensing data.

This work performed at JPL under a contract from NASA's Planetary Geology and Geophysics Program.

1. Gehrels, T. Astrophys. J, 123, 331-338, 1956

2. Hapke, B. W. Icarus, 67, 264-280, 1986

3. Shkuratov, Yu. SA-A.J., 27, 581-583, 1983

4. Hapke, B.W. Icanus, 88, 264-280, 1986.

5. Nelson, R. M., B. W. Hapke, W. D. Smythe, L. J. Horn. Icarus 131, 223-230, 1998,

6. Nelson, R. M., B. W. Hapke, W. D. Smythe, L. J. Spilker. Icarus, 147, 545-558, 2000.

7. Nelson, R. M., W. D. Smythe, B. W. Hapke, A. S. Hale. to appear in Planet. Space Sci, 2002. 

SPACE WEATHERING PROCESSES ON MERCURY S. K. Noble and C. M. Pieters, Brown University. Dept of Geological Sciences, Providence RI 02912. noble@porter.geo.brown.edu

Introduction: Like the Moon, Mercury has no atmosphere to protect it from the harsh space environment and therefore it is expected that it will incur the effects of space weathering [1]. These weathering processes are capable of both creating regolith and altering its optical properties $[1,2,3]$. However, there are many important differences between the environments of Mercury and the Moon. These environmental differences will almost certainly affect the weathering processes as well as the products of those processes. It should be possible to observe the effects of these differences in Vis/NIR spectra of the type expected to be returned by MESSENGER. More importantly, understanding these weathering processes and their consequences is essential for evaluating the spectral data retumed from MESSENGER and other missions in order to determine the mineralogy and the iron content of the Mercurian surface. Theoretical and experimental work has been undertaken in order to better understand these consequences $[4,5]$.

Mercurian Environment: Due to its higher impactor flux and greater density, Mercury will produce 13.5 times the melt and 19.5 times the vapor than is produced on the Moon per unit area [6]. Additionally, Mercury has a magnetic field that protects its surface from charged particles which severely reduces the solar wind flux at the surface [7]. Therefore, melting and vaporization due to micrometeorites are expected to dominate space weathering on Mercury with little or no solar wind sputtering effects [1]. Furthermore, agglutinitic glass-like deposits and vapur deposited coatings should be created faster and more efficiently on Mercury. The Mercurian environment is also notable for its extreme temperature range. Due to its slow rotation and proximity to the sun, equatorial regions of Mercury achieve temperatures above $700 \mathrm{~K}$ during the day, while nighttime temperatures fall below $100 \mathrm{~K}$.

The nanometer-scale metallic Fe particles (npFe ${ }^{0}$ ) that are ubiquitous in the rims and agglutirnates of lunar soil [8] (figure 1) should also be present on Mercury. Even for the endmember case where the surface of Mercury has no native $\mathrm{FeO}$, the iron brought in by meteorites should be sufficient to make the formation of $\mathrm{npFe}$ through vapor fractionation an important process on the planet. Amounts as small as 0.05 $\mathrm{wt} \% \mathrm{npFe}$ are enough to affect the optical properties [2].

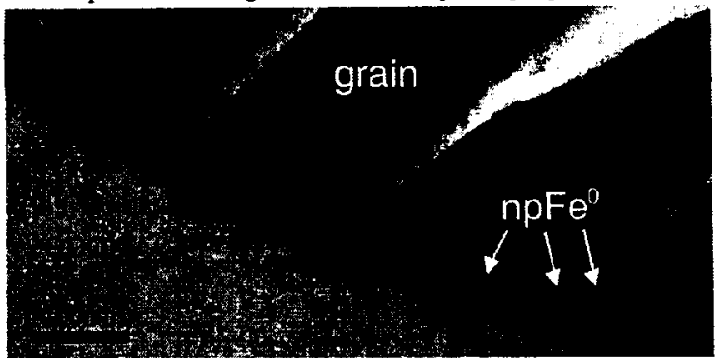

Figure 1. TEM bright field image of $\pi \mathrm{pFe}^{0}$-rich rim on a lunar soil grain

Size of $\mathrm{npFe}^{0}$ Particles: The size distribution of metallic Fe particles in a soil strongly controls the optical effects on the Vis/NIR spectrum. In lunar rims, these $n p F e^{0}$ particles range from $\sim 1-12 \mathrm{~nm}$ in dia. with an average of $\sim 3 \mathrm{~nm}$ [9] The smallest particles $(3-5 \mathrm{~nm})$ will tend to redden the soil while larger particles $(>10 \mathrm{~nm})$ will simply cause darkening $[9,10]$.

Ostwald Ripening: In figure 2 are shown the results of a $\mathrm{npFe} \mathrm{F}^{0}$ study in which $\mathrm{Fe}^{0}$ particles averaging about $8 \mathrm{~nm}$ in diameter were created and then heated for 10 hours at a range of temperatures [12]. The graph plots the final size of the particles vs. the heating temperature. This study is not directly applicable to Mercury because here iron particles are in grain to grain contact rather than occurring as isolated particles suspended in a glass matrix as in space weathering products. However, the experiment is useful because it demonstrates that this size of nanophase $\mathrm{Fe}^{0}$ particles may only be stable to about $200^{\circ} \mathrm{C}$. The majority of Mercury's surface reaches daytime temperatures significantly above $200^{\circ} \mathrm{C}$. In fact, during the course of a Mercurian day, the soil at the hottest parts of Mercury will stay above $400^{\circ} \mathrm{C}$ for about 2 weeks. This increased temperature may be enough to allow the $n p F e^{0}$ particles to grow significantly via Ostwald ripening, a process well known in material sciences [11]. Ostwald ripening is a process by which $n p \mathrm{Fe}^{0}$ particles in a glass matrix will tend to coarsen due to differences in free energy between curved surfaces.

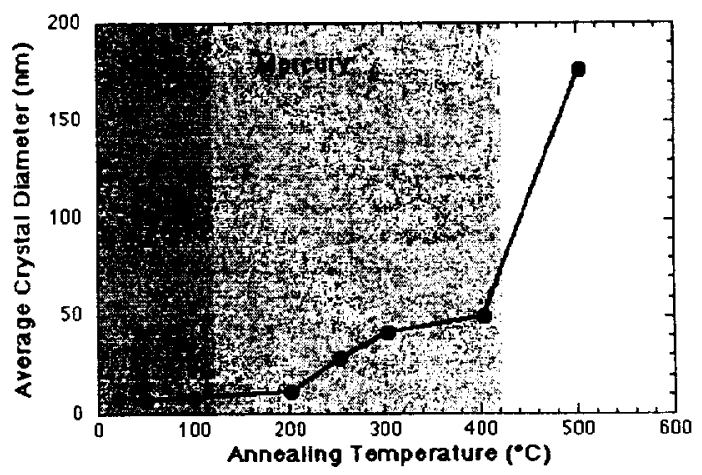

Figure 2. Size of $\mathrm{npFe}^{0}$ particles after annealing for 10 hours. Modified from Glieter, 1989 [12]. The shaded areas show the upper extent of the temperature regimes for the Moon and Mercury, respectively.

A vapor deposition experiment of Hapke et al. [13] demonstrated that heating $n p F e^{0}$-rich vapor coatings to a temperature of $650^{\circ} \mathrm{C}$ for just one hour is sufficient to remove a ferromagnetic resonance. This is hypothesized to occur because particles of $n \mathrm{Fe}^{0}$ have grown to be larger than the range that is measured by FMR techniques [4-33 $\mathrm{nm}$ in dia.[14]]. Thus, it appears that even for npFe particles suspended in a glass matrix, increased temperatures can result in significant grain growth.

Determining the rate of Ostwald ripening on Mercury is difficult due to a lack of directly relevant experimental data. The least constrained, and most important, variables are the diffusion coefficient of $\mathrm{Fe}^{0}$ into the glass matrix, which is strongly temperature dependent, and the surface energy (i.e. boundary between $\mathrm{npFe} \mathrm{e}^{0}$ and matrix), also somewhat temperature dependent. By considering a wide range of values for these, we have attempted to bound the possible range of grain growth through time that might occur on Mercury (figure 3). 


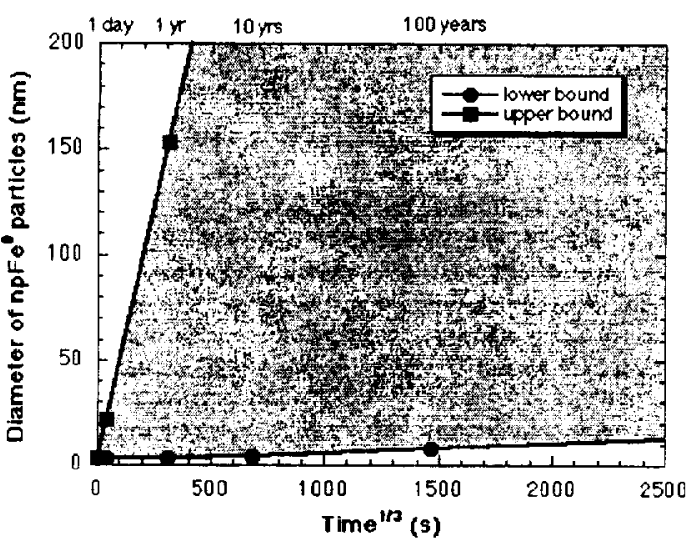

Figure 3. Possible range of effects of Ostwald ripening with time near the equator of Mercury. Lower bound assumes $D$ (diffusion coef.) $=10^{-19} \mathrm{~m}^{2} / \mathrm{s}, \sigma$ (surface energy) $=0.1 \mathrm{~mJ} / \mathrm{m}^{2}$, Upper bound assumes $D=10^{-17} \mathrm{~m}^{2} / \mathrm{s}, \sigma=100 \mathrm{~mJ} / \mathrm{m}^{2}$.

Obviously, these results cover a wide range of possibilities, however, even our most conservative estimates (lower bound) indicate that Ostwald ripening should have a significant effect on equitorial Mercurian soils, doubling the size of the $n p \mathrm{Fe}^{0}$ in a matter of centuries. Of course, with increasing latitude, less solar heating occurs and the thermal regime becomes much more lunar-like where Ostwald ripening will have little to no effect. Certainly polar regions that do not reach temperatures above $200^{\circ} \mathrm{C}$ (beyond $75^{\circ} \mathrm{N}$ or $\mathrm{S}$ for the hot poles, and $65^{\circ}$ for the warm poles) are not expected to be affected by Ostwald ripening as $n p F e^{0}$ particles appear to be stable at those temperatures [12].

Discussion: The size of $\mathrm{npFe}^{0}$ particles will be reflected in remotely acquired data. Ostwald ripening should result in larger $\mathrm{Fe}^{0}$ particles, on average, near the equator where the highest temperatures are reached. As noted earlier, small $\mathrm{npFe} \mathrm{e}^{0}$ particles cause reddening of the reflectance spectrum and larger particles result in darkening. If Ostwald ripening dominates over npFe $e^{0}$ production near the equator, we expect the spectral continuum to become darker at lower latitudes as one approaches the equator and the increased heat allows for larger npFe ${ }^{0}$ particles. The continuum should be reddest at high latitudes, where Ostwald ripening has little or no effect and $n p F e^{0}$ particles remain small.

Our current spectral data set for Mercury, is limited. Most of our spectral data is telescopic [15], largely providing an integrated disk view, masking possible latitudinal variations, as well as regional differences. Also hidden are maturity differences that might be expected at young craters. Two bands of spectral data taken during the Mariner 10 flyby confirms that spectral differences exist on regional and local scales [16], however this dataset does not have the spectral or spatial resolution necessary to see the effects discussed above. Recently, the surface was mapped over the wavelength range $550-940 \mathrm{~nm}$ at roughly $200 \mathrm{~km}$ resolution by the Swedish Vacuum Solar Telescope [17]. Originally, latitudinal variations were removed in the calibration of this dataset, however, some of the data has been reprocessed to allow spectral variations with latitude to be observed [18] (figure 4). Despite significant scatter, it does appear that, as predicted, there is a positive correlation between spectral redness and increasing latitude.

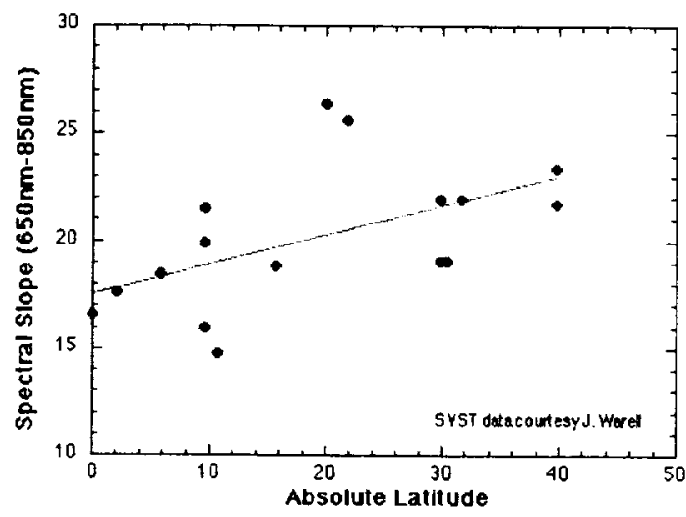

Figure 4. Spectral slope from 650 to $850 \mathrm{~nm}$ vs absolute latitude for 16 features on Mercury. Reprocessed Mercury data provided by $\mathrm{J}$. Warell.

Future Work: To address some of the questions raised here more quantitatively, we have begun two sets of experiments. Both use porous silica gel powders impregnated with nanophase metallic iron, following the technique of Allen et al [19]. The first of these experiments will investigate the optical effects of particle size of $n p F e^{0}$. Because the pore size, and by analogy the $n p F e^{0}$ particle size, can be controlled, these powders are well suited for studying the optical properties of differing sizes of $n p F e^{0}$ particles. We expect to derive from this experiment the particle size at which $n p F e^{0}$ transitions from causing spectral reddening to darkening. The second experiment involves heating the npFe $\mathrm{f}^{0}$-bearing powders for extended periods of time (days to weeks) and measuring the growth rate of the $\mathrm{npFe}{ }^{0}$ particles. This will allow us to better constrain the range of expected effects of Ostwald ripening. In combination, these experiments will provide a foundation for understanding the optical effects of $\mathrm{npFe}^{0}$ in Mercury's environment. This knowledge, in tum, is essential to evaluate mineralogy and iron content from the Vis/NIR spectra expected to be obtained by MESSENGER.

References: [1] Hapke B. (2001) JGR, 106, 10039. 10073. [2] Noble S. K. et al (2001) Meteoritics \& Planet. Sci., 36, 31-42. [3] Pieters C. M. et al (2000) Meteoritics \& Planet. Sci., 35, 1101-1107. [4] Noble S. K. and Pieters C. M. (2001) Mercury Workshop, 68-69. [5] Noble S. K. and Pieters C. M. (2002) Astron. Vestnik, submitted. [6] Cintala M. J. (1992) JGR, 97, 947-973. [7] Hartle R. et al (1975). JGR, 80, 3689-3693. [8] Keller L. P. and Clemett S. J. (2001) LPSCXXXII, ab\# 2097. [9] Keller L. P. et al (1998) New Views of the Moon, 41. [10] Britt D. T. and Pieters C. M. (1994) GCA, 58, 3905-3919. [11] Lifshitz I. M. and Slyozov V. V. (1961) J. Phys. Chem. Solids, 19, 35-50. [12] Gleiter H. (1989) Progress in Mat. Sci., 33, 223-315. [13] Hapke B. et al. (1994) Science, 264, 1779-1780. [14] Housley R. M. et al (1976) PLPSC $7^{\text {th }}, 13-26$. [15] Vilas F. (1988) Mercury, Univ. of AZ Press, 59-76. [16] Robinson M. S. and Lucey P. G. (1997) Science, 275, 197-199. [17] Warell J. and Limaye S. S. (2001) Planet. Space Sci., 49, 1531-1552. [18] Warell, J. (2002) Personal Communication. [19] Allen C. C. et al (1996) LPSCXXVII, 13-14.

Acknowledgments: The guidance of Yan Liang and Paul Hess is much appreciated. NASA support (NAG54303 ) is gratefully acknowledged. 
NEW ESTIMATES OF LUNAK OPPOSITION SPIKE CHARACTERISTICS WITH CLEMENTINE DATA. V. Omelchenko, V. Kaydash, Yu. Shkuratov. Kharkov Astronomical Observatory, 35 Sumskaya, Kharkov, 61022, Ukraine. E-mail: omelchenks@astron.kharkov.ua

Introduction: Several techniques have been used to estimate the opposition spike parameters of the lunar surface by Clementine data [1-3]. The simplest way is averaging many images that contain the spacecraft shadow point (zero-phase-angle point) neglecting albedo variations, which anyhow remain after the averaging [1]. More accurate way is to use a phaseratio image, one component (one initial image) of which contains the zero-phase-angle point and the other one is obtained at larger phase angles [2]. This technique is more precise as the phase-ratio images almost do not contain albedo variations. A drawback of both these approaches is strong dependence of final results on camera characteristics drift that was considerable during the mission. The third technique suggests to use images acquired ilmost simultaneously and containing the zero-phase-angle point [3]. It allows to avoid problems with the drift of camera parameters. With that technique lograrithmic derivatives of the phase function for eacly point of imaged surface can be calculated. For this purpose D/C colorratio (from UVVIS camera images) is used [3]. A shortcoming of the technique is availability of slight variations of the $\mathrm{D} / \mathrm{C}$ color-index $([)=0.95 \mu \mathrm{m}$ and $\mathrm{C}$ $=0.90 \mu \mathrm{m}$ ) over the ratio image. We estimate here an opposition spike parameter for several small mare and adjacent highland regions of the lunar surface suggesting a new technique (that develops the third one), which is also free of the UVVIS camera characteristics drift. We have studied a near-equatorial region placed in the latitude range $8^{\circ} \mathrm{N}-8^{\circ} \mathrm{S}$ at the $0.5^{\circ} \mathrm{E}$ longitude (the lunar near-side ).

Technique and results: The lementine spacecraft was moving along the polar orbit and imaging the lunar surface near the nadir point. Thus images taken successively at the same spectral band overlap almost by $30 \%$. The phase-ratio images for the overlapping areas do not burden with slight variations of the $\mathrm{D} / \mathrm{C}$ color-index. We use the overlapping images containing the zero-phase-angle point or its neighbor. This point is placed at different sites of the images (or even beyond the images), not close to each other, as it was in case of the $\mathrm{D} / \mathrm{C}$ color-ratio images. Therefore, we may not determine the derivatives of the phase function directly. Nevertheless, the'se phase-ratio images are very informative and can he used to estimate lunar opposition spike characteristics.

Initially we followed the algorithm of Clementine data calibration described in [4]. We used the radio- metric calibration only (including the flat field correction). Thus the counts in the images used are proportional to the reflectivity values. Totally eight pairs of UVVIS camera images in each filter covered the lunar surface region indicated above were used. The ratio of two initial images in one filter represents a set of quotients $F\left(\alpha_{1}\right) / F\left(\alpha_{2}\right)$ for a set $\Delta \alpha=\alpha_{1}-\alpha_{2}$, where $F(\alpha)$ is the phase function, $\alpha$ being the phase angle. Having taken the equation for the phase function in the form $F(\alpha)=\exp (P(\alpha))$, where $P(\alpha)=k_{1} \alpha+k_{2} \alpha^{2}$ $+\ldots$, the coefficients $k_{i}$ can be found. For this purpose we use the experimental quotients and the leastsquares method.

Consider the suggested method using as an example a pair of images comprising the north-eastern portion of Sinus Medii (North is below). Initial images we used is presented in Fig. 1 a and $b$ (the craters Bruce and Blagg are seen in Fig. 1 a). The white lines show borders of the common portion of the images. The ratio of the initial images is shown in Fig. 2 . The levels of the equal values of $|\Delta \alpha|$ for each image element are shown in Fig. 3. As it turns out the simplest presentation for the phase function, $F(\alpha)=$ $\exp (k \alpha)$, is quite enough, since the values $|\Delta \alpha|$ are rather small, they do not exceed a few degree. We minimize the sum $\Sigma\left[\ln \frac{F\left(\alpha_{1}\right)}{F\left(\alpha_{2}\right)}-k\left(\alpha_{1}-\alpha_{2}\right)\right]^{2}$ in order to estimate $k$. We have estimated the phase function slope $k$ for eight pairs of images covering both mare surface (Sinus Medii) and highlands southward of the mare region. We use images taken in four spectral bands, A, B, C, and D. All these provides data to study correlation between the phase function slope $k$ (at first several degrees of phase angle) and reflectivity of the lunar surface. This correlation is shown in Fig. 4. As one can see there is no clear dependence of $k$ on reflectivity.

Discussion: The brightness opposition spike of regolith-like surfaces is formed by two mechanisms: the shadow-hiding mechanism and the mechanism of coherent backscatter enhancement. The slope of phase angle function formed by the first mechanism decreases with growth of albedo, as the shadowing effect is suppressed by multiple scattering, whose role increases with albedo increase. Unlike this, the coherent backscatter mechanism predicts increasing the slope with growth of albedo. Our results show that there is no dependence at all. Three explanations for 
NEW ESTIMATES OF LUNAR OPPOSITION SPIKE CHARACTERISTICS. Omelchenko et al.

this can be suggested: (a) the radiometric calibration is not perfect enough to obtain reliable estimates of photometric slopes; (b) the lunar surface in the region under study is too dark (actually albedo varies in the range $7-18 \%$ ) to provide a detectable demonstration of both mechanisms; and (c) as the mechanisms affect the photometric slope in the opposite manner, they can mutually be compensated. It is difficult to make a choice between this three cases. In principle one can have surprises with calibration of Clementine UVVIS data, but we consider that the probability to obtain a "zero" result due to instrumental errors is very low. The second reason is also unlikely, as almost ten years ago B. Hapke [5] showed that some sample of the lunar regolith definitely reveal the coherent backscatter effect. The third reason seems to be more attractive, though it demands studies, in particular, with laboratory or computer modeling.

Anyway, in future it is challenging to use the above-described technique for data, which will be obtained during ESA Smart-1 mission to construct phase ratio images using overlapping Clementine and Smart-1 frames.

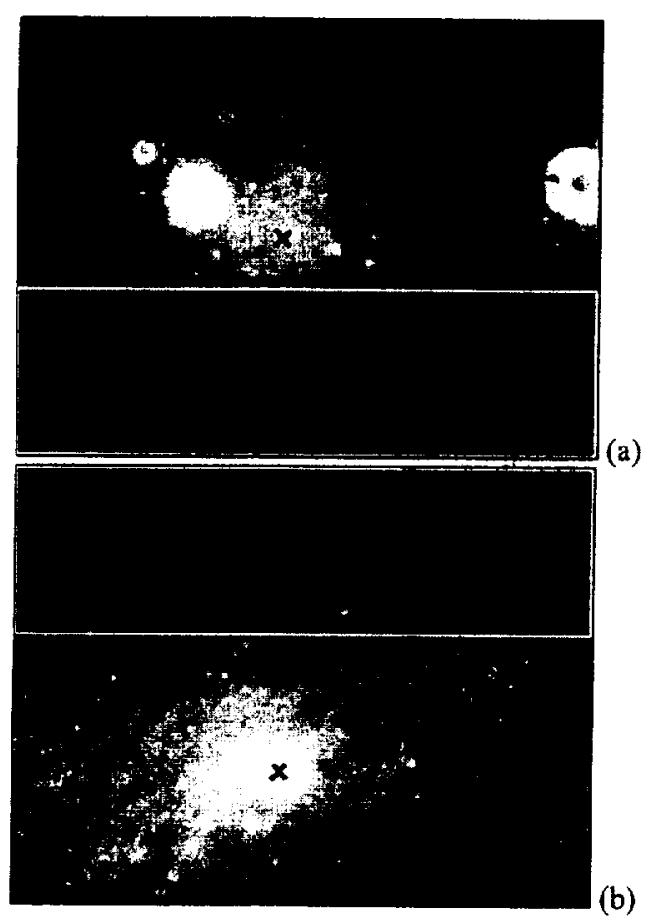

Fig. 1 a,b. The region under study. Crosses indicate the zero-phase-angle points. Overlapping regions have white outlines.

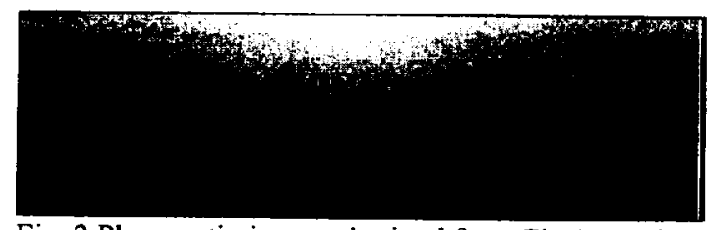

Fig. 2 Phase-ratio image obtained from Fig $1 \mathrm{a}$ and $\mathrm{b}$.

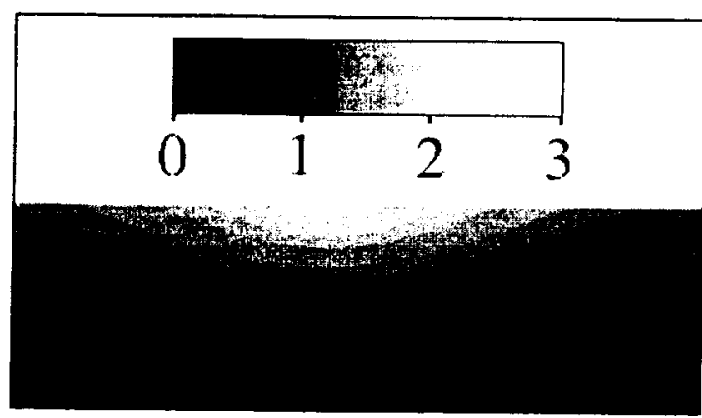

Fig. 3. The isolines of the equal $|\Delta \alpha|=\alpha_{1}-\alpha_{2}$ values (scale is given in degrees) for the phase ratio image.

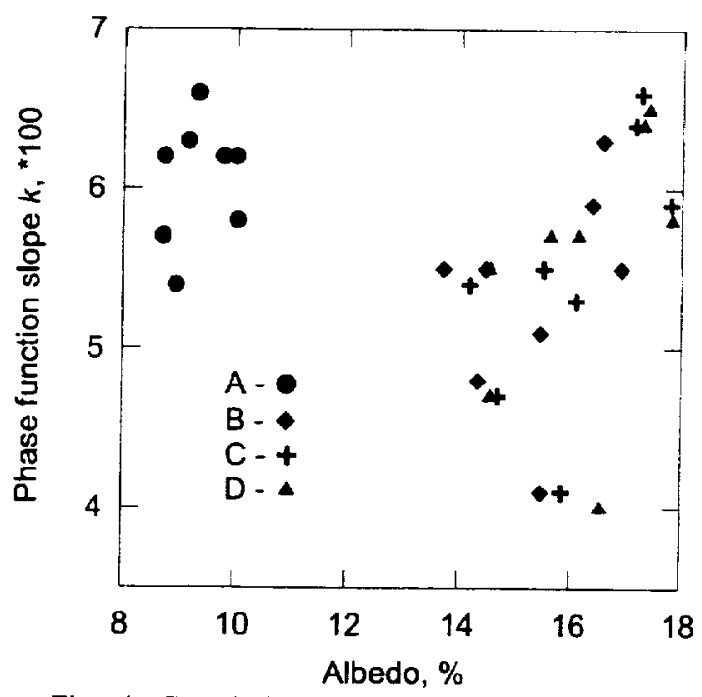

Fig. 4. Correlation between the phase function slope $k$ and albedo of the lunar surface (data for UVVIS filters $A, B, C, D$ ).

Acknowledgments: This study was supported by INTAS grant \#2000-0792.

References: [1] Hillier J. et al. (1999) Icarus, 141, 205-225. [2] Kreslavsky M. A. et al. (2000) JGR Planets 105, 20,281 - 20,295. [3] Shkuratov Yu. et al. (1999) Icarus 141, 132-155. [4] McEwen A. et al. (1998) Lunar and Planet. Sci. 29-th. LPI Houston. Abstract \# 1466. [5] Hapke, B. et al. (1993) Science, 260, 509-511. 


\section{GROUNDBASED RADAR INVES TIGATIONS OF ASTEROIDS AND PLANETARY SATELLITES.}

S. J. Ostro, Jet Propulsion Laboratorv. California Institute of Technology (ostro@ reason.jpl.nasa.gov).

Radar is a powerful source of iıformation about the physical and dynamical properties of solar system bodies. Radar-detected targets include the Moon, Mercury, Mars, Venus, Phobos, Io, Europa, Ganymede, Callisto, Titan, lapetus, Saturn's rings, eight comets, and 179 asteroids (75 main-belt and 104 near-Earth). This talk offers a perspective on the disc-integrated rallar properties of solar system bodies and then turns to what radar remote sensing can tell us about asteroids using spatially-resolved measurements.

In dual-polarization observations, which have been routine for two decades [1], echo power is received in the same circular polarization as transmitted (the SC sense) as well as the opposite (OC) sense. SC/OC is a measure of the near-surface structural complexily at scales near the wavelength (usually $3.5 \mathrm{~cm}$ for Goldstone and $13 \mathrm{~cm}$ for Arecibo). If $\mathrm{SC} / \mathrm{OC}$ is near zero, thin single reflections from smooth surface facets dominate the scattering process, but constraints on the scattering prosess and physical characteristics become increasingly ambiguous and model-dependent as SC/OC increases to a few tens of percent or larger, because such values indicate increasing contributions from single reflections from rough surfaces and/or multiple scattering.

Among all the radar-detected planetary bodies in the solar system, Europa, Ganymede, and Callisto have the most unusual radar properties $[2,3]$. Their reflectivities are huge and their circular polarization ratios exceed unity (Figure 1); that is, in contrast to the situation with other targets, the scattering largely preserves the handedness, or helicity, of the transmission. As Hapke $[4,5]$ first realized, the key to understanding the satellites' radar behavior is the coherent backscatter effect. The icy satellites' echoes are due not to external surface reflections but to volume scattering from within the satellites' regoliths, which the radar sees as an extremely transparent, disordered random medium. The high radar transparency of ice compared with that of silicates permits deeper radar sounding, longer photon path lengths, and higher order scattering from regolith heterogeneities. Coherent backscattering yields strong echoes with SC/OC larger than unity because the incident wave's direction is randomized before its helicity is randomized and before its power is absorbed.

The SC/OC estimates for several near-Earth asteroids (NEAs) approach Callisto's value [6], but their radar albedos are not large, so those asteroids' echoes do not suggest significant coherent backscattering, much less the presence of ice. Large values of SC OC probably can be explained by many, very different structural configurations. On the other hand, the fact that SC/ $)$ C estimates for NEAs range from near zero to near unity establishes that the surfaces of these objects are extremt-ly variegated and in some cases much more complex than any seen by spacecraft that have landed on Eros (whose $\mathrm{SC} O \mathrm{OC}=0.3$, near the NEA average [7]), the Moon, Venus, or Mars.

For most asteroids, radar is the only groundbased remote sensing technique that can achieve useful spatial resolution. Delay-Doppler imaging with $\sim 20$-m resolution is now routine whenever the echoes are strong enough, and images with adequate orientational coverage can be used to make three-dimensional models. As reviewed by [8] radar has revealed both stony and netallic objects, principal-axis and complex rotators, very smooth and extremely rough surfaces, objects that must be monolithic and objects that probably are not, spheroids and highly elongated shapes, contact-binary shapes, and binary systems (which apparently constitute - 1/6 of NEAs as large as $0.2 \mathrm{~km}[9]$ ).

Recently it has been shown that knowledge of physical properties is tightly coupled to long-term prediction of close approaches of NEAs to Earth, because of "Yarkovsky" accelerations from thermal re-radiation of absorbed solar energy[10]. Someday our ability to ascertain whether some asteroids could threaten Earth centuries in the future may rest on how well we understand their global distributions of photometric and thermal properties.

References: Ostro S. J. (2002) Planttary radar astronomy, in The Encyclopedia of Physical Science and Technology, 3rd Edition (R. A. Meyers, ed.), Academic Press, 12, 295-328. [2] Ostro S. J. et al. (1992) JGR 97, 18227-18244. [3] Black G. J. et al. (2001) Icarus 151, 160-166. [4] Hapke B. (1990) Icarus 88, 407-417. [5] Hapke B. and Blewett D. (1991) Nature 352, 46-47. [6] Benner L. A. M. et al. (1997) Icarus 130, 296-312. [7] C. Magri C. et al. (2001) MAPS 36, 1697-1709. [8] Ostro S. J. et al. (2002) Asteroid radar astronomy, in Asteroids III (W. Bottke, A. Cellino, P. Paolicchi, and R. P. Binzel, eds.), Univ. of Arizona. [9] Margot J. L. et al. (2002) Science 296, $1445-1448$. [10] Giorgini J. D. et al. (2002) Science 296, 132-136. 


\section{RADAR PROPERTIES}

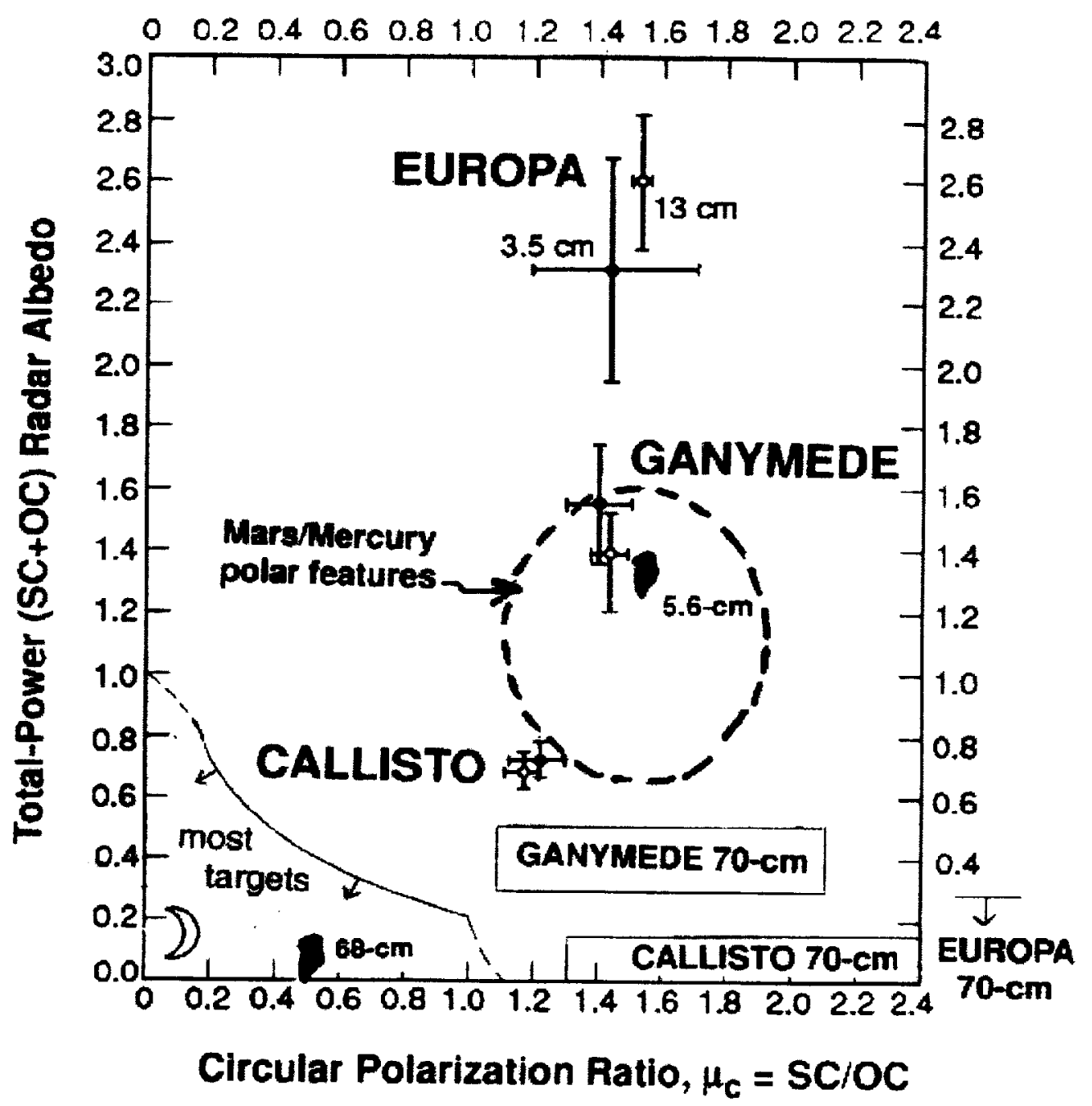

Figure 1 
SIZE-DEPENDENT SCATTERING PROPERTIES OF PLANETARY REGOLITH ANALOGS. J.L. Piatek', B. Hapke', R.M. Nelson', A. S. Hale ${ }^{2}$, and W.D. Smythe ${ }^{2}{ }^{1}$ Department of Geology and Planetary Science, University of Pittsburgh, ${ }^{2}$ Jet Propulsion Laboratory.

Introduction: Particles in a planetary regolith can be treated as independent scatterers when their size is large compared with the wavelength of light. The nature of this scattering, however, is poorly understood as the particle size approaches and becomes smaller than the wavelength. In order to understand this interaction better, the reflectances of well-sorted particulate samples of known composition were measured. This reflectance data was then analyzed to determine how scattering properties change with particle size.

Method: The samples used in this study were 13 aluminum oxide abrasive powders, 6 iron oxide pigment powders, and 4 precipitated calcium carbonate powders. The aluminum oxides range in size from 0.1 to $30.09 \mu \mathrm{m}$, the iron oxides range in size from 0.15 to $2.5 \mu \mathrm{m}$, and the calcium carbonates range in size from 0.06 to $3.0 \mu \mathrm{m}$. SEM analysis has been done to verify not only the particle size of the samples, but the size distribution and the particle morphology. In addition, $x$-ray diffraction has been used to identify the iron oxide mineralogy. The instruments used to obtain the reflectance data are the long and short arm goniometric photopolarimeters (or goniometers) at the Jet Propulsion Laboratory. Both instruments measure the intensity of red laser light reflected from a sample in 8 senses of polarization ( 4 linear, 4 circular). The long arm goniometer is capable of measuring phase angles from 0.05 to $5^{\circ}$ with a minimum phase angle (' $\mathrm{g}$ ') increment of $0.01^{\circ}$. Nelson et al. (2000) [1] has previously presented the data for the aluminum oxide samples, as well as describing the instrument. The short arm goniometer is capable of measuring phase angles from 4 to $160^{\circ}$ with a minimum phise angle increment of $0.5^{\circ}$. For the data presented bere, the incidence angle ('i') is $60^{\circ}$, making the maximum possible phase angle $140^{\circ}$. Data taken with this instrument for a number of samples has previously been published [2].

For each instrument, a run begins by measuring a $99 \%$ reflectance standard for calibration purposes. The standard is measured at $\mathrm{i}=0^{\circ}, \mathrm{g}=5^{\circ}$ in all 8 polarizations. The sample is then measured at the incidence angle used for data collection and $\mathrm{g}=5^{\circ}$ in all polarizations. The results are used to calculate normal albedo and to calibrate the sample data. The resulting phase curves from both instruments cover both very large and very small phase angles. These curves are then subjected to a series of theoretical fits to attempt to determine scattering parameters such as single particle scattering function, single scattering albedo, the intensities of the coherent backscatter and shadow-hiding opposition effects, the half-width half-max (HWHM) of the coherent backscatter opposition surge, the transport mean free path, and the scattering cross section of the particles. An improved suite of bidirectional re- flectance equations from Hapke, 2002 [3] are used to obtain the fits, and an example is shown in Figure 1. The single particle scattering function (' $\mathrm{p}(\mathrm{g})$ )' and the single scattering albedo ('w') are first estimated by assuming $\mathrm{p}(\mathrm{g})$ can be approximated by a 2 nd order Legendre polynomial expansion, and then fitting the phase curve with a Hapke equation fit. If a satisfactory fit cannot be obtained, additional approximations for $p(g)$ are explored, including higher order Legendre polynomial expansions and double Henyey-Greenstein functions (expanded in Legendre polynomials). The fit is allowed to vary from the data where the coherent backscatter opposition surge is apparent in the circular polarization ratio data. These fits are then refined by adding in terms to account for both the shadow-hiding and coherent backscatter opposition surges: the coherent backscatter term contains a variable that measures the HWHM of this opposition surge. Previous work has noted that this HWHM varies with particle size $[4,5]$. The HWHM is used to then calculate the transport mean free path, and this used to determine the scattering cross section of the particles. The single particle scattering function is used to obtain the average scattering angle $\langle\theta\rangle$, which can then be related to the extinction and absorption cross-sections. These two parameters are normally used as inputs for Mie scattering calculations, and can be used to compare these lab results with parameters commonly used in planetary remote sensing.

Results: Contrary to theoretical expectations, the aluminum oxide powders all exhibit coherent backscatter peaks with HWHM's of less than $2^{\circ}$ for both very large and very small particles. Coherent backscatter opposition surges are apparent in the short arm data only in the $2.1,3.2$, and $4.0 \mu \mathrm{m}$ sized aluminum oxides. On the other hand, the iron oxide powders all exhibit coherent backscatter peaks with HWHM's of about 20 degrees, showing no dependence on particle size. Likewise, the calcium carbonate samples also exhibit coherent backscatter peaks widths with no apparent dependence on particle size. The single particle phase functions of all the powders exhibit well developed backscatter lobes, regardless of what approximation is used to represent $p(g)$ (see Figures 2,3 and 4 ). Over the range of phase angles studied, none of the powders had the strong forward scattering lobes predicted by theoretical models based on scattering by spheroids. As the particle size decreases, there is no indication of the change from Mie-type forward scattering to Rayleigh scattering predicted by models that assume the particles scatter independently. 


\section{References:}

[1] Nelson, R.M. et al. 2000. Icarus 147, 545-558.

[2] Nelson, R.M. et al. 1998. Icarus 131, 223-230.

[3] Hapke, B. 2002. Icarus 157, 523-534.

[4] Hapke, B. 1993. Theory of Reflectance and Emittance Spectroscopy. $455 \mathrm{pp}$.

[5] Mishchenko, M.I. 1993. Astrophys. J. 411, 351-361.

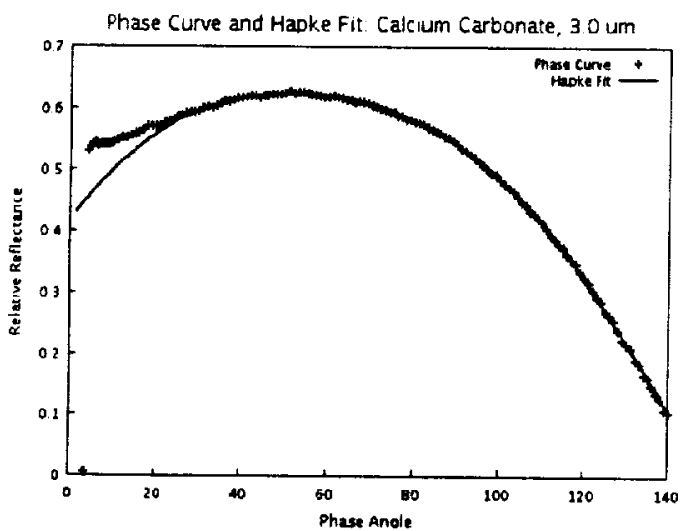

Figure 1: Example of Hapke equation fit to calibrated phase curve data. Single particle scattering function is approximated by a $3^{\text {rd }}$ order Legendre polynomial expansion.

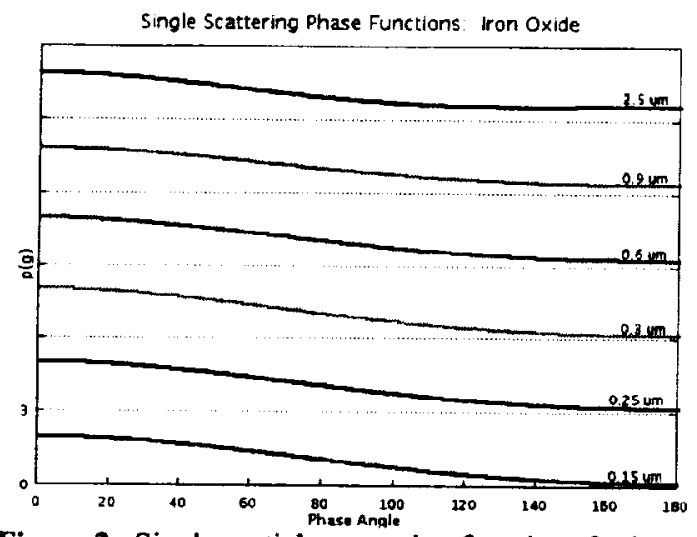

Figure 2: Single particle scattering functions for iron oxide samples, using $2^{\text {nd }}$ order Legendre polynomial expansion. Curves are offset, and the y-axis scale is the same for each.

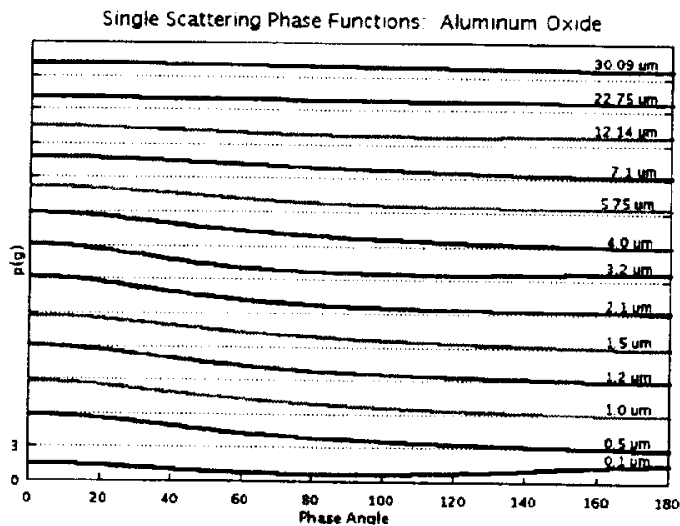

Figure 3: Single particle scattering functions for aluminum oxide samples, using $5^{\text {th }}$ order double HenyeyGreenstein function expanded in Legendre polynomials. Curves are offset, and the $y$-axis scale is the same for each.

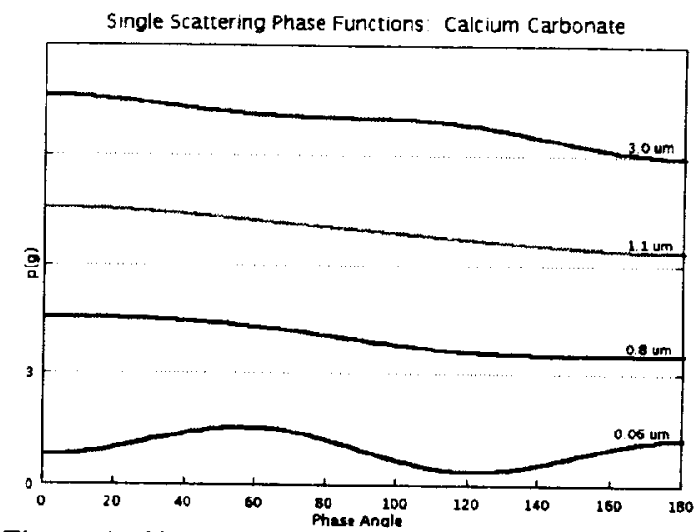

Figure 4: Single particle scattering functions for calcium carbonate samples, using $3^{\text {rd }}$ order Legendre polynomial expansion. Curves are offset, and the $y$ axis scale is the same for each. 
Lessons Learned from Lunar EXPloration: The Moon Continues to SuRPrise Us. C. M. Pieters, Dept. of Geological Sciences, Brown Univ., Providence, RI 02912 (pieters@mare.geo.brown.edu)

The Early Years (1960's and 1970's). It has often been said that exploration of the Moon was implemented backwards. The Apollo national initiative of the 1960 's resulted in landing men on the Moon at six sites and returning samples to earth-based laboratories for study. These unique and valuable samples brought a quantum leap in understanding the age and evolution of the solar system. Simultaneously, the former Soviet Union developed unmanned sample return methods and successfully returned lunar samples from three additional locations. Although these remarkable accomplishments were envisioned as the dawning of a golden era of space exploration, the emphasis in the US quickly turned to developing the Shuttle and using unmanned missions to explore more distant horizons. Management essentially considered lunar exploration closed. In spite of continuous efforts from an everdwindling community of lunar scientists, no phase of detailed study of the Moon with advanced sensors was implemented. A well-instrumented lunar polar orbiter was proposed countless times, but never flown. Lunar exploration went into hibernation for decades.

The Reawakening (1990's). Lunar exploration was re-initiated almost by accident. [In reality, it was sneaked in by never-give-up lunar scientists.] The Galileo spacecraft flew by the Moon twice to get extra energy for its trip to Jupiter. Both times the teams were convinced it was valuable to test and calibrate systems using the Moon. This was the first time relatively modern CCD sensors were used in lunar orbit and Galileo provided the first new orbital spectroscopic data for the Moon [1]. A few years later DoD decided to test a group of miniaturized cameras designed to measure fast moving targets. For political reasons they needed to go outside near-Earth space and approached NASA to ask if there was scientific interest in the data to be produced for the Moon and an asteroid. The lunar science diehards were elated, and soon a Clementine science team was formed to plan and implement science data [2]. After countless calibration issues (some still ongoing) the first global topography [3] and global multispectral imagery data sets were produced for the Moon [e.g. 4]. The only planned mission to the Moon in this second wave of exploration was Lunar Prospector, the cheapest Discovery mission ever selected by NASA. This little mission provided the first global assessment of elemental abundance (especially $\mathrm{Th}, \mathrm{K}$, $\mathrm{Fe}, \mathrm{Ti}, \mathrm{H})[5]$.

The Surprises. The combined new pulse of lunar data knocked the socks off complacent planetary sci- entists who thought they understood the Moon. This is not to say important research had not continued via lunar sample studies and telescopic measurements of the lunar nearside during the dark decades. The origin of the Moon and the Earth/Moon system through a violent planetismal encounter was well on the way to being understood [6] But our perception and understanding of the Moon changed forever with this little bit of new data from relatively modem sensors. Three particularly important surprises are highlighted below.

SPA, the Biggest Basin in the Solar System. Although several of the diehard lunar enthusiasts had long argued for a big basin on the lunar farside [e.g. 7], the South Pole-Aitken basin (SPA) is missing from most commercial lunar maps produced using Apollo era data. This remarkable oversight reflects the limited character of the data available. The first digital images from Galileo changed everything, however. Not only was the basin rim readily recognized, but the basin interior had a distinctively low albedo and exhibited spectral properties indicating that it was rich in mafic minerals [1]. Subsequent data from Clementine illustrated the dramatic topography ( $12 \mathrm{~km}$ deep!) [3] and unusual mineralogy exposed in the interior [8]. It became apparent that SPA may be the largest documented basin in the solar system [3,9], and the deepseated material of the interior represents a terraine on the Moon that is quite distinctive and unlike the lithologies documented with samples returned from the nearside [10].

Thorium Hot Spot. The global elemental data from Lunar Prospector confirmed that the SPA interior is iron-rich (midway between feldspathic highlands and mafic mare basalt). One of the most unexpected results, however, was the very well-defined pattem of radiogenic materials, almost all of which are associated with the nearside Imbrium basin and its deposits. Since such elements (KREEP material) are expected to be concentrated in the residual of primordial lunar differentiation, it is not surprising that a large basin tapped such a concentration. The surprise is that the pattern is only associated with the Imbrium basin, which is not only smaller than SPA but also occurred near the end of the period of basin forming events. This highly localized concentration of radiogenic (incompatible) elements indicates there are enormous gaps in our understanding of the first few hundred million years of planetary evolution and differentiation.

Polar $H$ (water?). The extremely low volatile content of lunar samples was one of the first characteris- 
tics noted for returned lunar samples (along with their ancient age). Thus, the hint of polar ice by Clementine [11] and the unquestioned discovery of hydrogen concentration at the lunar poles by Prospector [12] has led to great optimism that water resources may indeed exist in the permanently shadowed regions of the Moon. This counter-intuitive concept, although reasonable in theory [13], is made more credible by the discovery of extensive volatile (perhaps ice) deposits in craters at Mercury's poles [14].

Meanwhile, back at the ranch.... One of the more perplexing puzzles presented by the returned lunar samples was that lunar soils don't really look like lunar rocks at visible to midIR wavelengths. This is a key concept for remote sensing studies, since data obtained by remote sensing provides the only information on local, regional, and global properties of the Moon and soils invariably dominate remotely acquired data. Understanding soil processes is thus critical and it has taken close to 30 years to resolve. Reinvestigating lunar soils with modern instruments in earth-based laboratories identified the importance of nano-scale processes that occur on soil grains when exposed to the space environment $[15,16]$. Although the seeds were planted from the beginning [17], it is the cumulative insight from detailed laboratory and theory investigations that allowed identification of the dominant role of nanophase reduced iron in altering optical properties [18] and provided the modern paradigm of regolith processes active in the space environment.

The Critical Role of Samples. What can we learn from our experience of using samples in exploration of the Moon? Some aspects seem déjà vu. Mars dust and soil doesn't look anything like crustal rocks (SNC meteorites); nor do typical meteorites look like the surfaces of most asteroids, that might represent likely parent bodies. Nevertheless, it appears to be assumed that remote sensing and in-situ measurements of other bodies will provide sufficient information on the distribution and type of materials to provide key geologic context needed to unravel planetary evolution. The lunar experience provides a few cautionary insights.

The first is that without samples of lunar soil in our laboratories we would not have been able to understand (read interpret) the available remote sensing data sufficiently to discern the primary character of the Moon. Try to imagine our understanding without the samples. We would have detected the presence of pyroxene and would have noted that it appears to be more abundant at fresh craters for some reason. The distribution of iron from Lunar Prospector and the Apollo orbital experiments might have led to the supposition that all the maria are basaltic, but there would be no conclusive evidence. We would puzzle about the weakness of pyroxene features in soil, perhaps hypothesizing it is due to a high degree of mysterious contamination. The highlands are older than the maria (based on superposition and crater density), but we would have no reference for actual age. We would know the highlands are low in iron, but might think the higher albedo is due to smaller particles resulting from more impacts. The weaker absorptions in the highlands might also be due to the greater age, and hence contamination. Such musings would all be nonsense, a gross misconception based on ignorance of fundamental information about lunar soils and their relation to primary rocks.

A second related example is that without samples of key lunar lithologies (high-Ti and low-Ti basalt, feldspathic breccias, KREEP, Mg-suite), we cannot discern the inter-relations between different rock types, so essential to formulating the basic geologic history (magma ocean with mafic mantle cumulates and crustal anorthositic cumulates). In addition to petrologic relations, the precision needed for diagnostic minor and trace elements and isotopic abundances requires Earth-based laboratories. The importance of the Thorium Hot Spot has special meaning to lunar scientists because it most likely represents concentration of the magma ocean residuum in a very localized region of the Moon. The unique mafic-rich interior of SPA presents a challenge to what we think we know about the structure and composition of the lunar lower crust and mantle. If we knew in the 1960's what we know now, we would certainly have argued long and hard with the engineers to obtain a representative sample of SPA. As a footnote, it is also recognized that, at least for the Moon, soils provide a remarkably representative suite of materials of local and foreign origin [19].

References. 1. Belton, M. J. S., et al. (1992) Science 255(5044): 570-576; Belton, M. J. S (1994).Science 264(5162): 1112-1115. (2) Nozette, S. et al. (1994). Science 266: 1835-1839. (3) Zuber, M. T., et al. (1994) Science 266: 1839-1843. (4) Eliason, E. M. et al. (1999). NASA PDS CDRom Archive, CL-4001-4078. (5) Lawrence, D. J. et al. (1998). Science 281: 1484-1489. (6) Hartman, W. K. et al. (1986). Origin of the Moon. LPI, 781pp. (7) Wilhelms, D. E. (1987). USGS PP 1348, pp302. (8) Pieters, C. M et al. (2001) JGR 106(E11): 28,001-28,022. (9) Spudis, P. D et al. (1994) Science 266: 1848-1851 . (10) Jolliff, B. L. et al. (2000) JGR 105(E2): 4197-4216. (11) Nozette, S., C. et al. (1996). Science 274: 1495-1498. (12) Feldman, W. C., et al. (2000). JGR 105(E2): 4175-4195 (13) Amold, J. R. (1979) JGR 84: 5659-5668. (14) Harmon, J. K et al. (1994). Nature 369: 213 215. (15) Keller, L. P. and D. S. McKay (1997) Geochim. et Cosmochim. Acta 61 (11): 2331-2341. (16) Taylor, L. A et al. (2001) JGR106(E11): 27,985-28,000. (17) Hapke B. (2001) JGR 106,10039-10073. (18) Pieters, C. M et al. (2000) Meteoritics \& Planetary Science 35: 1101-1107. (19) Wood, J. A., J. S. Dickey, et al. (1970). Apollo 11 Lunar Sci. Conf., Vol. 1.; Jolliff et al. (2002) LPS XXXIII \#1156. 


\section{INTERCONTINENTAL BISTATIC RADAR TEST OBSERVATION OF ASTEROID 1998 WT24.}

S. Righini ${ }^{1}$, S. Poppi ${ }^{2}$, S. Montebug $10 i^{3}$, M. Di Martino ${ }^{4}$, L.Saba ${ }^{5}$, M.Delbo ${ }^{6}$, S.Ostro ${ }^{7}$, J. Monari ${ }^{8}$, M. Poloni ${ }^{9}$ A. Orlati $i^{10}$, A. Zaitsev ${ }^{11}$.

1, 2, 3, 8, , , ${ }^{10}$ IRA - CNR, via Fiorentina Aia Cavicchio, Medicina (Bo), Italy; ${ }^{4,5,6}$ INAF - Osservatorio Astronomico di Torino, Pino Torinese (To), Italy; NASA-JPL, Pasadena (California, USA); ${ }^{11}$ IRE RAS, Moskow, Russia.

Abstract: We describe the first intercontinental planetary radar test performed in Italy observing the near Earth asteroid (NEA) 33342 (1998 WT24) in December 2001 by means of the bist tic configurations Goldstone (California, USA)-Medicina (Italy) and Evpatoria (Ukraine)-Medicina.

The experiment goal was to characterise the system for realtime radar follow-up observations of NEAs and artificial orbiting debris, in the frams work of a feasibility study which aims at using the Sardinia Radio Telescope, at present under construction, ilso as a planetary radar facility.

We report the preliminary results of the radar observations carried out by the IRA-CNR (Istituto di Radioastronomia - Consiglio Nazionale delle Ricerche) and the OATo (Osservatorio Astronernico di Torino) groups, aimed at exploring the scientific potentials of a new space radar program, using thr existing facilities in Italy.

The planetary radar technique is uniquely capable of investigating geometry and surface properties of various solar system objects [1], demon itrating advantages over the optical methods in its higl spatial resolution and ability to obtain three-dimensionit images.

A single radar detection allows to obtain extremely accurate orbital elements, improving the instantaneous positional uncertainties by orders of magnitude with respect to an optically determined orbit. Radar is a powerful means to spatially resolve NEAs by measuring the distribution of the echo power in time delay (range) and Doppler frequency (line-of-sight velocity) with extreme precision in each coordinate, as it provides detailed information about the target physical properties like size, shape, rotation. near-surface bulk density and roughness and internal density distribution[1].

The Medicina $32 \mathrm{~m}$ antenna had been successfully used for the first time as the receiving part of a bistatic configuration during a test experiment (September 2001) held to check the capabilities of the entire data acquisition system. This test was possible thanks to the collaboration undertaken with the Evpatoria radar station, and consisted in the observation of the ETALON-1 low orbit satellite.

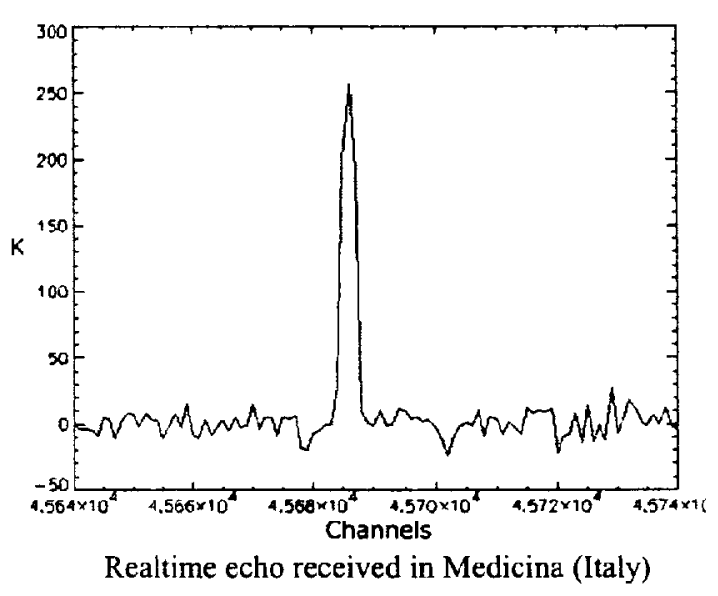

References: [1] S.J. Ostro (1993) "Planetary Radar Astronomy" Rev. Modern Phys., vol.65, no.4 

COMPOSITIONAL REMOTE SENSING OF ICY PLANETS AND SATELLITES BEYOND JUPITER Ted L. Roush, NASA Ame's Research Center, MS 245-3, Moffett Field, CA 94035-1000 (troush@mail.arc.nasa.gov)

Introduction: The peak of the solir energy distribution occurs at visual wavelengths and falls off rapidly in the infrared. This fact, improvements in infrared detector technology, and the low surface temperatures for most icy objects in the outer solar system have resulted in the bulk of telescopic and spacecraft observations being performed at visual and near-infrared wavelengths. Such observations, begun in the early 1970's and continuing to present, have provided compositional information regarding the surfaces of the satellites of Satum and Uranus, Neptune's moon Triton, Pluto, Pluto's moon Charon, Centaur objects, and Kuiper belt objects. Because the incident sunlight penetrates the surface and interacts with the materials present there, the measured reflected sunlight contains information regarding the surface materials, and the ratio of the reflected to incident sunlight provides a mechanism of identifying the materials that are present.

Fink and Sill [1] and Roush [2] present the reflectance spectra of various ices. An initial identification of the species present on solar system objects can be provided by comparison with these laboratory spectra. More detailed information, such as relative abundances grain sizes of surface constituents, requires more detailed models.
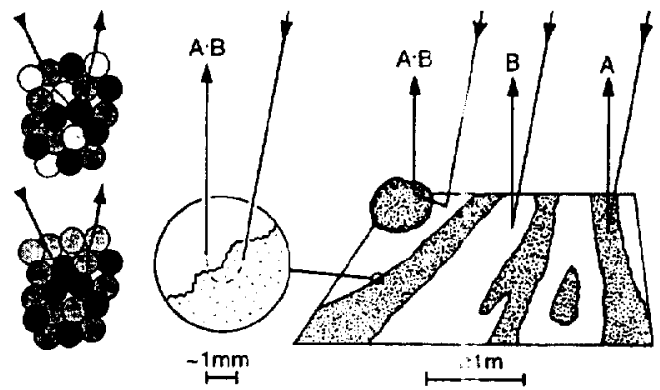

Figure 1. The cartoon on the right [j/ illustrates spatial mixing of spectra where the reflectan' $c$ is dominated by photons encountering individual materials. Along some pathways a component of intimate miving remains. The upper-left cartoon illustrates intimate spectral mixing where the reflectance is dominated by photons that encounter several materials. The low's-left cartoon illustrates intimate spectral mixing with a surface layer.

Various mathematical models are used to characterize the chemical and physical nature of the surfaces under investigation. They can also be used to extend the results of laboratory studies to different mixtures and particle sizes that are not directly investigated.
Spatial mixtures (see Fig. 1) describe photons reflected from the surface that are dominated by interac. tions with individual surface species. The total surface reflectance is given by $R_{T}=X_{1} \times R_{1}+X_{2} \times R_{2}+\cdots$ $+X_{n} \times R_{n}$, where $X$ is the areal extent, and $R$ the reflectance of each component, respectively, and it is required that $\Sigma X_{i}=1$. Exampies of these types of surfaces are individual rocks or outcrops.

In intimate, or "granular", mixtures (see Fig. 1) the surface reflectance is a non-linear function of the reflectances of each component because a photon encounters several grains, due to multiple scattering, before exiting the surface. Examples are individual minerals that are contained in rocks and planetary soils and regoliths.

A series of papers summarized in Hapke [4] developed an approximation to the intimate mixture case allowi the reflectance to be calculated from the optical properties of the surface components. This important contribution allowed the quantitative estimation of the relative abundances and grain sizes of surface components and, given the appropriate observations, some information regarding the surface physical structure. Table 1 summarizes some of these efforts applied to objects in the outer solar system.

Table 1. Outer Solar System Surfaces Modeled Using Hapke Theory

\begin{tabular}{lll} 
Object & \multicolumn{1}{c}{ Property } & \multicolumn{1}{c}{ Reference } \\
\hline Europa & T & 5 \\
Ganymede & T & 5 \\
Callisto & T, C & $5,[6-8]$ \\
Enceladus & T & 5 \\
Tethys & T & 5 \\
Dione & T, C & 5,9 \\
Rhea & T, C & 5,9 \\
lapetus & T, C & $5,[10-11]$ \\
Rings & T & 5 \\
Miranda & C & 12 \\
Ariel & T, C & 5,13 \\
Umbriel & T & 5 \\
Titania & T, C & $5,[13-14]$ \\
Oberon & T,C & $5,[13-14]$ \\
Triton & T, C & {$[15-16],[17-19]$} \\
Pluto & T, C & $20,[21-25]$ \\
Charon & T, C & $26,[23,26-30]$ \\
Trans- & & \\
Neptune & C & {$[31-33]$} \\
objects & & \\
\hline
\end{tabular}

$\mathrm{T}=$ Temperature, $\mathrm{C}=$ composition 
Figure 2, after Cruikshank et al. [32], is an illustration of the application of Hapke modeling to the interpretation of the spectrum of the Centaur Pholus. In the model the surface is represented by a spatial mixture with one area being carbon black and the other an intimate mixture of components.
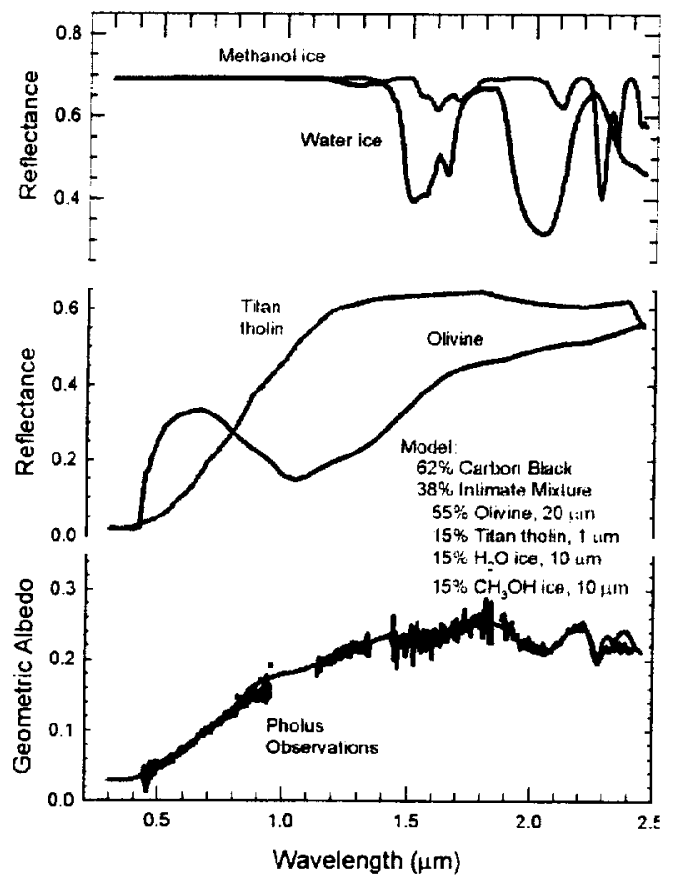

Figure 2. Comparison of a Hapke model of the surface with the measured spectrum of Pholus. At the top is a calculated spectrum of each pure material.

Molecular mixtures occur at atomic scales where one atom or molecule is substituted into the crystalline structure of a host material, for example, $\mathrm{CH}_{1}$ in $\mathrm{N}_{2}$. On the basis of subtle variations in the positions of methane bands, Owen et al. [21] and Cruikshank et al. [23] suggested that on Pluto the methane ice exists in two reservoirs: one relatively pure methane and the other mixed in nitrogen. Doute et al. [24] modeled Pluto's surface as a complex mixture including vertical layering (see Fig. 2).

The long list of Table 1 may erronously lead one to conclude that compositional interpretation of icy surfaces in the outer solar system is readily available via application of Hapke's [4] theory. Most ices are nonabsorbing at visual wavelengths and the visual colors of the various icy objects located in the outer solar system clearly demonstrate that non-ice materials are present. Some optical constants are available for limited materials exhibiting a range of colors at visual wavelengths [e.g. 34-35]. However, the majority of these have been determined with the material at ambient terrestrial conditions. How these characteristics change at conditions more appropriate to the outer solar system is largly unknown, although some information is becoming available [36]. Additionaly, optical constants for a variety of other candidate materials, e.g. silicates, are sorely lacking at visual and near-ir wavelengths. It is clear that there is still ample laboratory work to undertake.

From an observational perspective, the ability to achieve more unique interpretative solutions would benefit from spectral observations over a wide range of viewing geometries. Such observations are essentially impossible from ground-based observatories and await spacecraft measurements.

I gratefully acknowledge research support from NASA's Planetary Geology \& Geophysics Program

References: [1] Fink, U., and G.T. Sill, in Comets, L.L. Wilkening, Ed., Univ. of Ariz. Press, 1983 [2] Roush, T.L., $/ G R$., $106,33315,2001$ [3] Singer, R.B. and T.B. McCord, Proc. Lunar Planet. Sci. Conf. 10th, 1835. [4] Hapke, B., Theory of Reflectance and Emittance Spectroscopy, Cambridge Univ. Press, 1993 [5] Grundy, W.M. et al. Lcarus, 142, 536, 1999. [6] Roush, T.L. et al. /carks, 86, 355, 1990 [7] Calvin, W.M. and R.N. Clark, Icarzs, 89, 305, 1991 [8] Calvin, W.M. and R.N. Clark, /carus, 104, 69, 1993 [9] Benedix, G.K. et al. BAAS, 30, 1101, 1998 [10] Wilson, P.D. and C. Sagan, $\mathcal{G} R, 100,7531,1995$ [11] Owen, T.C. et al. Kcarus, 149, 160, 2001 [12] Bauer, J.M. et al. Kcarus, in press, 2002 [13] Roush, T.L. et al. LPSC XXZY, \#1636 1998 [14] Roush, T.L. et al. LPSC XXZX, \#16321998 [15] Tryka, K.A. et al. SCience, 261, 751, 1993 [16] Grundy, W.M. et al. Icarus, 105, 254, 1993 [17] Cruikshank, D.P. et al., Science, 261, 742, 1993 [18] Quirico, E. et al. /carus, 139, 159, 1999 [19] Cruikshank, D.P. et al. Icarus, 147, 309, 2000 [20] Tryka, K.A. et al. Icarus, 1/2, 513, 1994 [21] Owen, T.C., et al. Science, 26I, 745, 1993 [22] Grundy, W.M., and U. Fink, Icarus, 124, 329, 1996 [23] Cruikshank et al. Pluto and Charon, S.A. Stem and D.J. Tholen, Eds., U. Arizona, 1998 [24] Doute, S. et al. Kcarus, 142, 421, 1999 [25] Grundy, W.M. and M.W. Buie, Icarus, 153, 248, 2001 [26] Buie, W.M. and W.M. Grundy, Kcarus, 148, 324, 2000 [27] Roush, T.L. Icarus, 108, 243, 1994 [28] Roush, T.L. et al. Kcarus, 1/9, 2]4, 1996 [29] Brown, M.E. and W.M. Calvin, Science, 287, 107, 2000 [30] Dumas, C. et al. $A J, 121,1163,2001$ [31] Wilson, P.D. and C. Sagan, Kcarus, 107, 288, 1994 [32] Cruikshank, D.P. et al. Icarus, 135, 389, 1998 [33] Poulet, F. et al. Kcarus, submitted, 2002 [34] Khare, B.N. et al. lcarus, 60, 127, 1984 [35] Khare, B.N. et al. Icarus, 103, 290, 1993 [36] Roush, T.L. and J.B. Dalton, LPSC XXXIII \#/532, 2002. 
SIMULATION OF SPACE WEATHERING BY NANOSECOND PULSE LASER IRRADIATION: SPECTRAL CHANGE AND TEM-ESR CONFIRMATION OF NANOPHASE IRON PARTICLES. S. Sasaki $^{1}$, E. Kurahashi', K. Nakamura ${ }^{2}$, T. Hiroi ${ }^{3}$, C. Yamanaka ${ }^{4}$, 'Dept. Earth \& Planet. Sci., The University of Tokyo, 7-3-1 Hongo, Bunkyo-ku, Tokyo 113-0033, Japan (sho@eps.s.u-tokyo.ac.jp, erika@space.eps.s.u-tokyo.ac.jp), ${ }^{2}$ Dept. Earth \& Planet. Sci., Kobe Iniversity, Kobe 657-8510, Japan, ${ }^{3}$ Dept. Geological Sci., Brown University, Providence RI 02912, USA., ${ }^{4}$ Dept. Earth \& Space Sci., Osaka University, Toyonaka , Japan.

Introduction: In 1975, while examining why lunar regolith becomes darker and redder with time, Bruce Hapke with colleagues advocated that the lunar soil grains should have been coated with vapor-deposited rim containing nanometer-sized iron particles [1]. High-velocity dust impacts and/or solar wind irradiations might produce ferrous silicate vapor. This process is called "space weathering". However, this hypothesis had been ignored until nanophase "ron particles were confirmed in lunar regolith grains by TEM $[2,3]$.

S-type asteroids, majority in asteroids, are believed to be parent bodies of ordinary chondrites, which are a large majority in meteorites. However, S-type steroids exhibit more overall depletion and reddening of spectra, and more weakening of absorption bands relative to ordinary chondrites. This spectral mismatch is also considered being caused by space weathering process. Recent observation by Galileo showed that fresh crater interior and ejecta on S-type Ida have the spectra closer to that of ordinary chondrites [4]. Sume small asteroids have intermediate spectra between S-type and Q-type (ordinary-chondrite like) spectra [5]. NEAR's X-ray spectrometer showed that S-type 433 Eros has elemental composition close to ordinary chondrites [6].

Pulse Laser Irradiation Simulating Dust Impacts: Although van de Graaf type electrostatic dust accelerators can accelerate submicrun dust particles to be more than $10 \mathrm{~km} / \mathrm{s}$, they can hardly irradiate enough number of dust impacts to simulate space weathering. Moreover such accelerators use conductive dust materials such as $\mathrm{Fe}, \mathrm{Ag}$, and $\mathrm{C}$, which would affect the optical property of targets. Therefore, to simulate the high-velocity dust impact heating, we irradiate powder and pellet samples of silicates by a pulse laser beam (1064 $\mathrm{nm}$ ) with pulse duration 6-8 $1 \mathrm{~s}$, which is comparable with a real dust impact $[7,8]$. The total irradiation energy in unit area is $240 \mathrm{~mJ} \mathrm{~mm}^{-2}$ for $30 \mathrm{~mJ}$ pulse energy. Energy deposition rate is about $10^{10} \mathrm{Wcm}^{-2}$, where evaporation and ion formation is strongly expected [9].

Laser-irradiated samples show significant reddening: reduction of spectra is much larger in a visible region than in a near-infrared regın $[7,8]$. We use olivine from San Carlos with 8.97 wt\% FeO, enstatite from Bamble, Norway, with $9.88 \mathrm{w} \% \mathrm{FeO}$ and hypersthene from Mantyharju, Finland, with 16.7 wt\% FeO.
Reflectances of olivines are more easily changed than those of pyroxenes [8] This is compatible with the compiled asteroid data: using areas of $1 \mu \mathrm{m}$ and $2 \mu \mathrm{m}$ absorption bands, olivine-rich asteroids have more reddened spectra than pyroxene-rich asteroids $[10,11]$.

In Fig. 1, spectra are compared with observed spectra of olivine-rich asteroids. 446 Aeternitas spectrum is close to the olivine spectrum after $30 \mathrm{~mJ}$ irradiation. The scaled spectra of irradiated olivine look like compatible with rather flat asteroid spectrum at wavelengths longward of $1.5 \mu \mathrm{m}$ [12]. A more reddened slope of 863 Benkoela spectrum is reproduced by that of five times $30 \mathrm{~mJ}$ irradiations at wavelengths shorter than $1.6 \mu \mathrm{m}$ although repeated irradiation produced continuous reddened slope at longer wavelengths.

We also obtained following results: (1) After twice irradiation of $30 \mathrm{~mJ}$ pulse laser scanning, $510 \mathrm{~nm}$ band of enstatite was weakened [13]. This corresponds to the change of iron oxidation state. This effect is also discussed on lunar sample data. (2) We irradiate the mixtures of olivine and pyroxene with various mixing ratio by the pulse laser. Although the irradiated spectra of Ol-Px mixtures depend on the mixing ratio, the alteration is not proportional to the ratio. Space-weathered spectra of Ol-Px mixtures can be predicted using the end-member spectra of them [14]. (3) We have confirmed spectral changes (darkening and reddening) on an ordinary chondrite sample and a serpentine sample.

Nanophase Iron Particles: To clarify microscopic cause of reflectance change, we observed the irradiated samples by high-resolution scanning electron microscopy (SEM) and transmission electron microscopy (TEM). Thin sections (about 80-100 nm thickness) of irradiated grains were observed by $200 \mathrm{keV}$ High-Resolution TEM equipped with EDS.

In the SEM image of irradiated olivine, there are observed a number of small holes which were formed through surface evaporation (Fig. 2). In the TEM image of a thin section of an olivine grain, a large number of nanophase particles (several to $20 \mathrm{~nm}$ ) are observed in the amorphous rim (Fig. 3(a)). These are crystalline iron particles $[15,16]$. These particles are responsible for optical properties changes such as darkening and reddening as proposed [1] and calculated [17]. These particles may be related to nanophase iron in lunar soils 
$[2,3]$. In the TEM image from a laser-irradiated enstatite sample, nanophase iron particles are observed in amorphous aggregates (Fig. 3(b)). Our results show that hydrogen is not necessary to form nanophase iron.

In order to quantify the amount of nanophase iron, ESR (electron spin resonance) is measured for irradiated pellet samples by a microwave cavity method where spins in the surface depth of 100 micron can be detected nondestructively [18]. Strong ESR signals, which derive from nanophase iron particles, are observed both in irradiated olivine and pyroxene samples (Fig. 4). Moreover, ESR intensities increase with the space weathering degree simulated as laser irradiation time.

References: [1] Hapke B. et al., (1975) Moon 13, 339-353, [2] Keller L. P. and McKay D. S. (1993) Science 261, 1305-1307. [3] Keller L. P. and McKay D. S. (1997) GCA. 61, 2331-2341, [4] Chapman C. R. (1996) Meteoritics \& Planet. Sci., 31, 699-725. [5] Binzel $R$, et al. (1996) Science 273, 946-848. [6] Trombka J. I. et al. (2000) Science 289, 2101-2105. [7] Yamada M. et al. (1998) Antarctic Meteorites XXIII, 173-176. [8] Yamada M. et al. (1999) Earth Planets Space 51, 1255-1265. [9] Kissel J. and Krueger F. R. (1987) Appl. Phys. A 42, 69-85. [10] Hiroi T. and Sasaki S. (1999) LPS XXX, \#1444. [11] Hiroi T. and Sasaki S. (2001) Meteoritics \& Planet. Sci. 36, 1587-1596. [12] Clark B. E. and Hiroi T. (1994) Bull. Am. Astron. Soc. 26, 1172. [13] Hiroi T. et al. (2001) Earth Planets Space 53, 1071-1075. [14] Kurahashi E. and Sasaki S. (2002) LPS XXXIII, \#1479. [15] Sasaki, S. et al. (2001) Nature 410, 555-557. [16] Nakamura K. et al. (2001) LPS XXXII, \#1547 [17] Hapke, B. (2001) J. Geophys. Res. 106, 10039-10073. [18] Ikeya M. et al. (1994) Rev. Sci. Instrum. 65, 3670-3672.

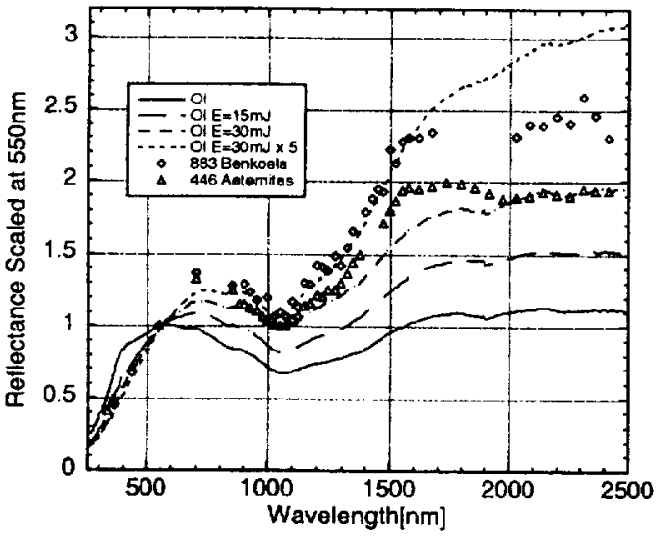

Figure 1. Reflectance spectra of olivine pellet samples before and after pulse laser irradiation. Olivine spectra scaled at $550 \mathrm{~nm}$. The scaled spectra are compared with asteroids 446 Aeternitas and 863 Benkoela.

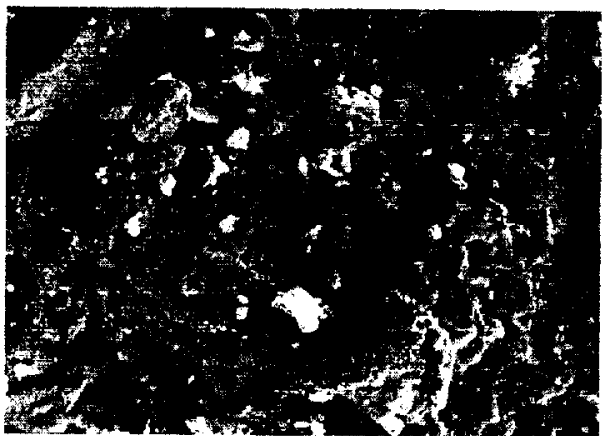

Figure 2. Backscattered SEM image of a pulse-laser irradiated olivine pellet surface $(30 \mathrm{~mJ} \times 10)$. Width of the figure is 40 micron.
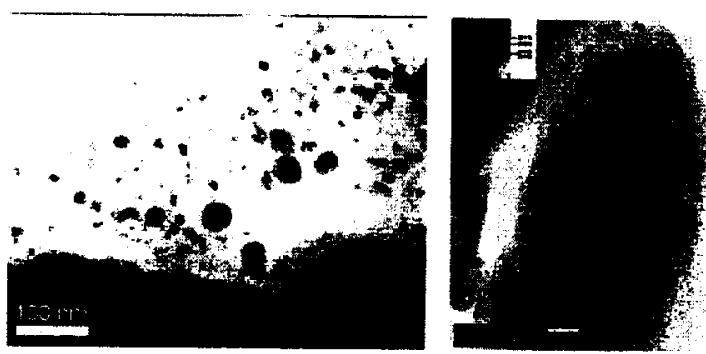

Figure 3 (a) A bright-field TEM image of a thin section from a laser irradiated olivine sample $(30 \mathrm{~mJ} \times 20)$. Nanophase iron particles whose size is several to $20 \mathrm{~nm}$ are within the olivine rim. (b) A bright-field TEM image of a thin section from a pulse-laser irradiated enstatite pellet sample $(30 \mathrm{~mJ} \times 20)$. Nanophase iron particles are embedded in amorphous aggregates.

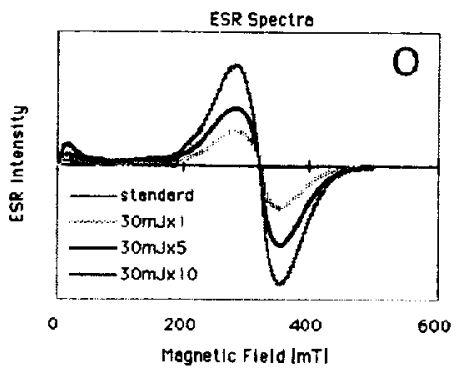

Figure 4. ESR spectra of olivine samples, which were irradiated by $0,1,5,10$ times at $30 \mathrm{~mJ}$. 
DEPENDANCE OF THE SPECTRAL BRDF OF SULFUR SURFACES WITH MATERIAL TEXTURE : LABORATORY MEASUREMENTS WTTH THE SPECTROPHOTO-GONIOMETER AT LPG. B. Schmitt ${ }^{\prime}$, N. Bonnefoy', O. Brissaud' and S. I'outé', 'Laboratoire de Planétologie de Grenoble, Bâtiment D de Physique, B.P. 53, 38041 Grenoble Cedex 9, France, email: Bernard.Schmitt@obs.ujf-grenoble.fr.

Introduction: The exploration of the surfaces of solar system bodies (planets, satellite, asteroids, ...) now systematically involves observations by imagers and imaging spectrometers over the UV, visible and nearinfrared ranges. All along the missions the light reflected by the surface is recorded under various illumination/observation geometries, often constrained by the spacecraft orbit. Large changes of reflectance and spectral contrast with phase angle are generally observed for a given area [1]. Also, contrary to terrestrial snow or ice fields, a tendency for backscattering behavior is widely found for the surfaces of the icy satellites [2].

In particular, lo's Jupiter satellite displays very strong contrast reversals between surface units at low and high phase angles [3]. This behavior seems to be linked to very different BRDF for the various materials constituting its volcanic surface dominated by sulfur compounds and $\mathrm{SO}_{2}$ frost. Several "i priori” interpretations have been proposed for this peculiar behavior but none has been theoretically modeled or tested experimentally.

The correct interpretation of the spectra produced by the imaging spectrometers under varied geometries first requires to be able to "correct" for these bidirectional effects. If sufficiently sampled, the BRDF of a planetary surface also potentially contain interesting textural information on its constituting materials. However, first of all laboratory investigations are clearly needed in order to understand the physical and textural parameters that control the BRDF. For that purpose a spectrophotogoniometer has been designed at LPG to measure the spectral bidirectional reflectance distribution function (SBRDF) of granular and compact geophysical surfaces. The instrument covers the solar spectrum, from 0.3 to 5 $\mu \mathrm{m}$, and enables a wide range of bidirectional configurations [4].

BRDF Measurements: We will first present the instrument and its performances. Then we will lay out the BRDF measurements performed over the visible spectral range on sulfur samples with various textures obtained by different physical processes that may occur on planetary surfaces: sublimation, thermal sintering, gas condensation, melting/solidification $[5,6]$.
Several sulfur powders exhibit a noticeable backscattering behavior, similar to some of the bright areas on lo. Such behavior, not predicted by the current single scattering models of large particles [7], seems to be linked with the sub-micron texture of the particles rather than with their average size and shape. During thermal sintering of these powders a more isotropic behavior develops. A very strong specular reflection is observed for melted/solidified sulfur but also, to a lower extend. for crystalline powders obtained by slow gas condensation at low pressure. All these directional behaviors are modulated by the strongly wavelength dependent absorption of sulfur.

Conclusion: These experiments and their comparison show that the BRDF signature, coupled with the spectral information, should help to discern between different textural states of a given surface material, and thus can give some clues on its formation and evolution processes. However adequate bidirectional sampling of the observations is requisite to correctly retrieve this information.

References: [1] Douté, S., Schmitt B., LopezGautier R., Carlson R., Soderblom L., Shirley J. and the Galileo NIMS Team (2001) Icarus, 149, 107-132. [2] Verbiscer A., and Helfenstein P. (1998) In Solar System Ices, (B. Schmitt, et al. eds.) Kluwer Acad. Publ., Dordrecht, Astrophys. Space Sci Lib., Val. 227, 157. 198. [3] Geissler, P.E.. , McEwen A., Phillips C., Simonelli D., Lopes-Gautier R. and Douté S. (2001) $/$. Geophys. Res. E, in press. [4] Brissaud O., Schmitt B., Bonnefoy N., Douté S., Fily M., Gundy W., and Rabou P. (2002) Appl. Optics, in preparation. [5] Bonnefoy N., Brissaud O., Schmitt B., Douté S., Fily M., Grundy W., and Rabou P. (2000). Remote Sensing. Rev. 19, 59-74. [6] Bonnefoy N. (2001) Thesis, UJF-LPG Grenoble. [7] Grundy, W.M., Douté S. and Schmitt B. (2000) J. Geophys. Res. E, 105, 29,291-29,314. 
BRDF OF SULFUR SURFACES: B. Schmitt, N. Bonnefoy, O. Brissaud, S. Douté
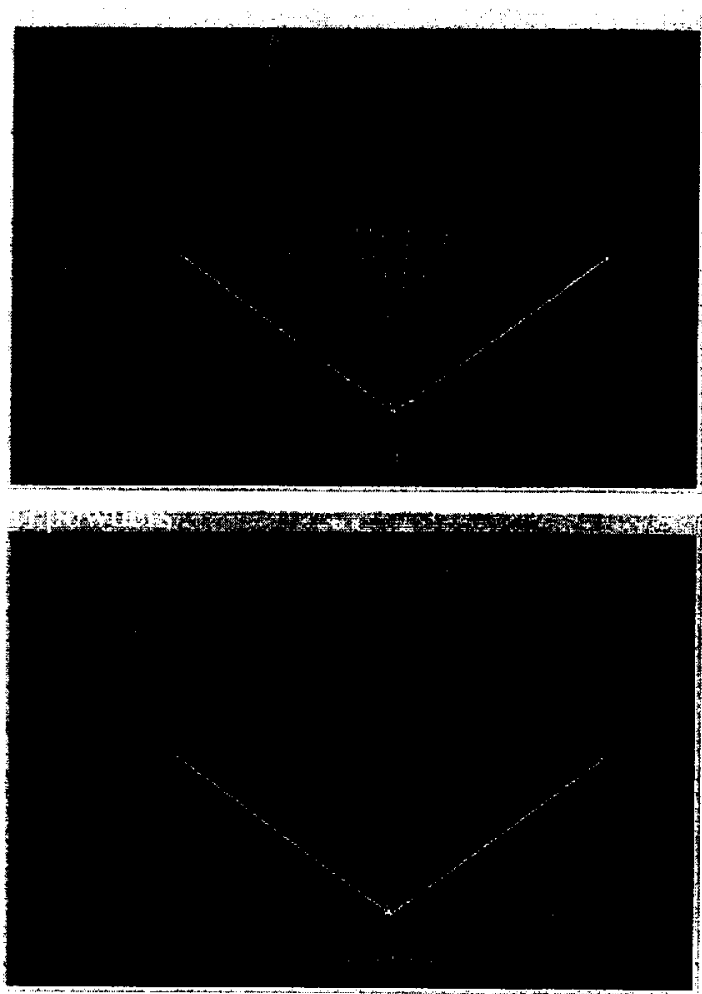

Figure I: Bidirectional Réflectance DistributionFunction (BRDF) in the principal illuminatin plane (source incidence: $60^{\circ}$ ) at $\lambda=492 \mathrm{~nm}$ for 4 sulfur samples: a) (top left) powders with "nominal" grain size of $\sim 40$ and $160 \mu \mathrm{m}, b$ ) (top right) thermaly sintered 40 $\mu \mathrm{m}$ powder $\left(12\right.$ days at $\left.90^{\circ} \mathrm{C}\right), \mathrm{c}$ ) (bottom left) gas phase condensed crystals under low pressure $\left.\left(210^{-2} \mathrm{mbar}\right), \mathrm{d}\right)$ (bottom right) melted $\left(125^{\circ} \mathrm{C}\right)$ and refrozen sample.
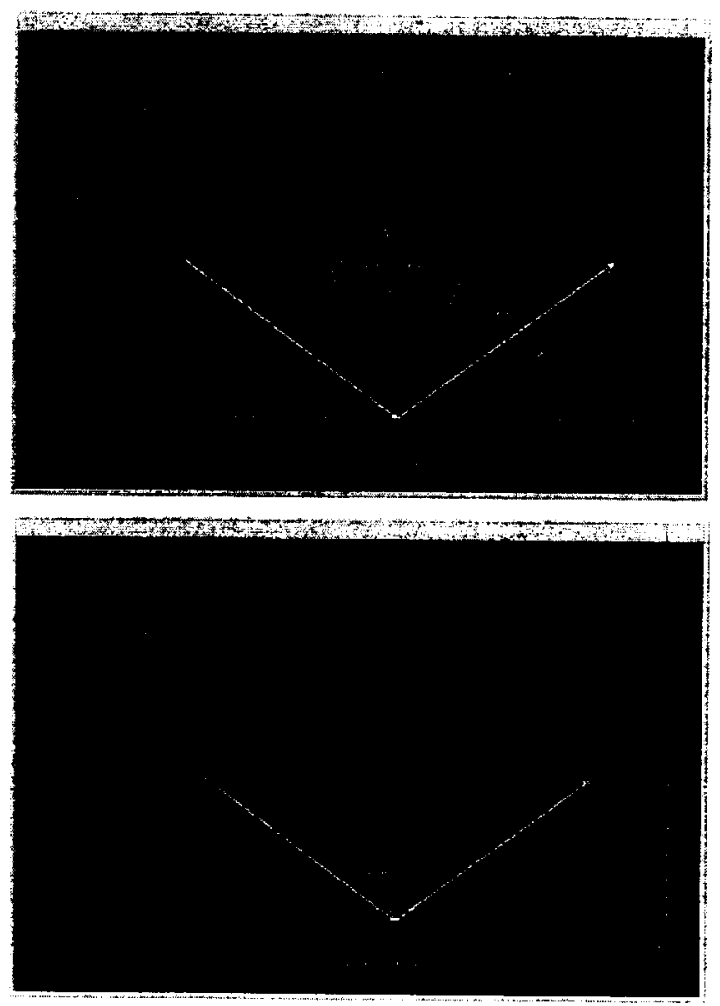

Acknowledgments: CNES and the French Programme National de Planétologie of CNRS (INSU) are acknowledged for their financial supports. 
Remote Sensing Analysis of the Terra Meridiani Region, Mars. F. P. Seelos IV and R. E. Arvidson, Department of Earth and Planetary Sciences, McDonnell Center for the Space Sciences, Washington University, St. Louis, MO 63130, seelos@wunder.wustl.edu.

Introduction: We report on a multifaceted investigation of remote sensing data for the Terra Meridiani region. Our study area extends \pm 10 degrees about the prime meridian and from -5 to +10 degrees in latitude, encompassing the largest of the hematite bearing regions identified on the surface of Mars [1]. A detailed investigation of this area was motivated by the likely selection of the hematite-bearing unit as the destination for one of the twin MER rovers. This study makes use of a variety of remote sensing data sets with the goal of characterizing the region to the fullest possible extent. The initial results from this body of work have contributed to the MER landing site selection process, and will aid in mission planning and operations if one of the rovers is sent to this region.

Remote Sensing Units Mapping: Through extensive examination and interpretation of MOC narrow angle and wide angle images [2], assisted by analysis of TES-derived bolometric albedo [3], TES-derived thermal inertia [4], MOLA-derived pulse width data (a surface roughness measure) [5], and the TES-based hematite index map [1], our study area was partitioned into five primary surface units (Figure 1).

The MOC image analysis also established that these units are set in a clear stratigraphic sequence [6]. The dissected cratered terrain exposed across the southern portion of the study area is mapped as the typical Noachian cratered terrain that pervades the southern hemisphere. The etched unit overlies the dissected terrain and is interpreted to be a severely degraded volcanic complex based on morphologic comparisons to known volcanic regions of Mars such as Athabasca. Notably, the etched unit has both an intermediate albedo and a very high thermal inertia, a combination that runs counter to the global norm. The hematite unit and plains unit overlie the etched unit and exhibit identical morphologic character. However, the plains unit lacks the elevated hematite index values that distinguish the hematite unit. The mantled cratered terrain extends to the north and is characterized by a dust mantling several meters thick. Our current model of the geologic history of this region terminates with the stripping back of the dust mantle still present to the north from the remainder of the study area, exhuming the plains, hematite, and etched units.

TES Hyperspectral Data: To facilitate the spectral analysis of the study region, a data cube with 256 pixels/degree and 138 spectral channels was constructed. Each TES spectrum was localized in the data cube according to the coordinates of the observation center, with each spectrum being relegated to a single spatial pixel. While this is not an accurate reproduction of the TES footprint on the surface, this configuration does allow for both spatially orientated analyses and the interpretation of individual spectra. Further, if the intent is to pass the spectral data to a deconvolution or analysis routine (as is the case here) this method of data localization guarantees that each spectrum is processed only once, instead of multiple times as would be the case if the spectrum were replicated in all of the spatial pixels covered by the actual footprint.

Spectral deconvolution/unmixing. The TES data cube was processed using a variant of the linear deconvolution algorithm advocated by the TES team [7]. This technique is capable of simultaneously deconvolving the atmospheric and surface signals and unmixing the derived surface spectrum in terms of a set of mineralogic endmembers. The dual deconvolution/unmixing procedure was performed with an endmember set consisting of six atmospheric endmember spectra provided by Bandfield, one blackbody spectrum to account for variations in spectral contrast, and -40 pure mineral spectra that collectively cover a wide range of possible surface mineralogies.

The deconvolution algorithm generates a series of resultant data cubes, all of which have the same spatial dimensions as the input. Resultant data cubes include: 1) the best-fit parameters for each spectral endmember (atmospheric and surface); 2) the parameter value assigned to the blackbody endmember and the RMS error of the spectral fit; 3 ) the best fit model spectrum over the wavenumber interval for which the linearity of the convolution holds; 4) the derived atmospheric spectrum and 5) the derived surface spectrum over the same wavenumber interval; 6) the normalized abundance of the atmospheric endmembers; and 7) the normalized abundance of the surface endmembers.

Data interpolation and map generation. Prior to the spatial interpolation of the deconvolution results, the RMS error band is used to eliminate low quality surface observations from consideration. While the data used to populate the original data cube was filtered for quality (e.g. dayside, nadir observations, without flagged errors), the deconvolution error for some orbits is anomalously large. This can be attributed to high atmospheric opacity at the time of observation as the linearity of the spectral convolution breaks down for high opacity observations [7]. High 
RMS errors are also common for dusty regions, an occurrence that can be explained by the nonlinear particle size dependency of emission spectra [8]. However, reducing the data density for dusty surface regions is an acceptable trade-off for eliminating highopacity orbits from consideration. The actual interpolation scheme that generates well-populated endmember maps also takes into account the RMS error of the deconvolution, and gives observations with low RMS errors greater weight. As a result, the statistical analysis of the interpolated maps will also be effectively error-weighted, with low-error observations attaining greater significance. Interpolated endmember maps can be appropriately combined to produce component maps of, for example, hematite abundance (Figure 2), or olivine abundance (Figure 3), for the entire region.

Results: The deconvolution/unmixing results and interpolated component maps provide valuable insight into the mineralogical variation among the surface units. Specific results include: 1) The mapping of the surface mineralogy served to bolster the validity of the unit boundaries, as all of the units are evident in the interpolated component maps. 2) Spectrally (and mineralogically) the etched unit differs significantly from the dissected unit only in clinopyroxene and glass abundance. This is a surprising result, given the differences these units exhibit in other characterizing quantities (e.g. albedo and thermal inertia). 3) The most striking correlation among the component maps is the association of iron-rich $\left(\sim \mathrm{Fo}_{31}\right)$ olivine with hematite (Figures 2-3). These components exhibit a positive correlation within all of the individual surface units, as well as across the study area as a whole. Table 1 gives the correlation coefficients for these components over various spatial regions.

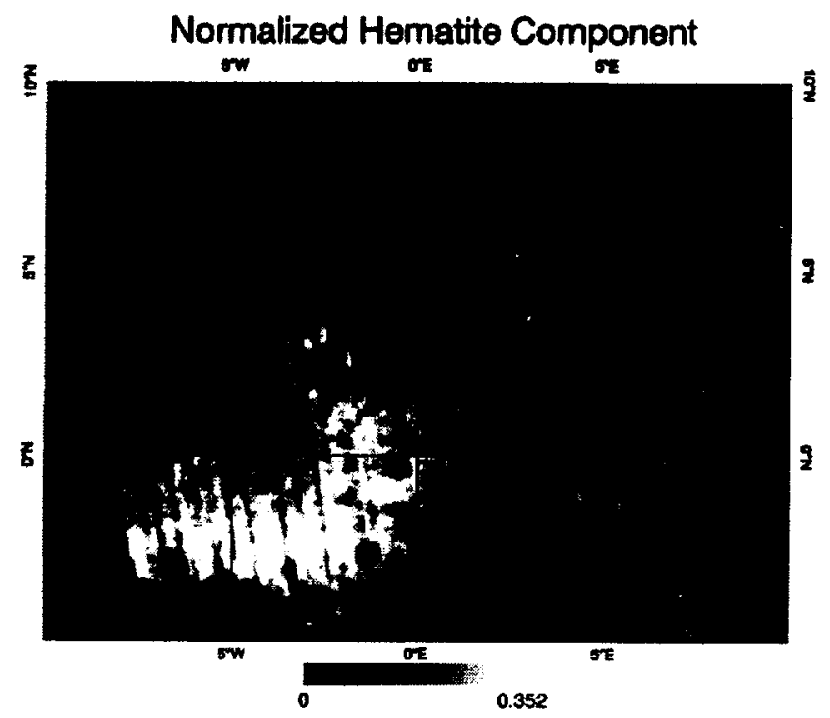

Figure 2. Normalized hematite component for the Terra Meridiani study region.
References: [1] Christensen P. R. et al. (2000) JGR, 105, 9623-9642. [2] Caplinger M. A. (2002) LPSC XXXIII, 1405. [3] Christensen P. R. Personal communication. [4] Jakosky B. M. et al. (2000) JGR, 105, 9643-9652. [5] Garvin J. B. et al. (1999) GRL, 26, 381-384. [6] Arvidson R. E. et al. (2002) in preparation. [7] Smith M. D. and Bandfield J. L. (2000) JGR, 105, 9589-9607. [8] Hamilton V. E. (1999) LPSC XXX, 2001

\section{Table 1. Correlation coefficient of olivine and} hematite components for selected regions.

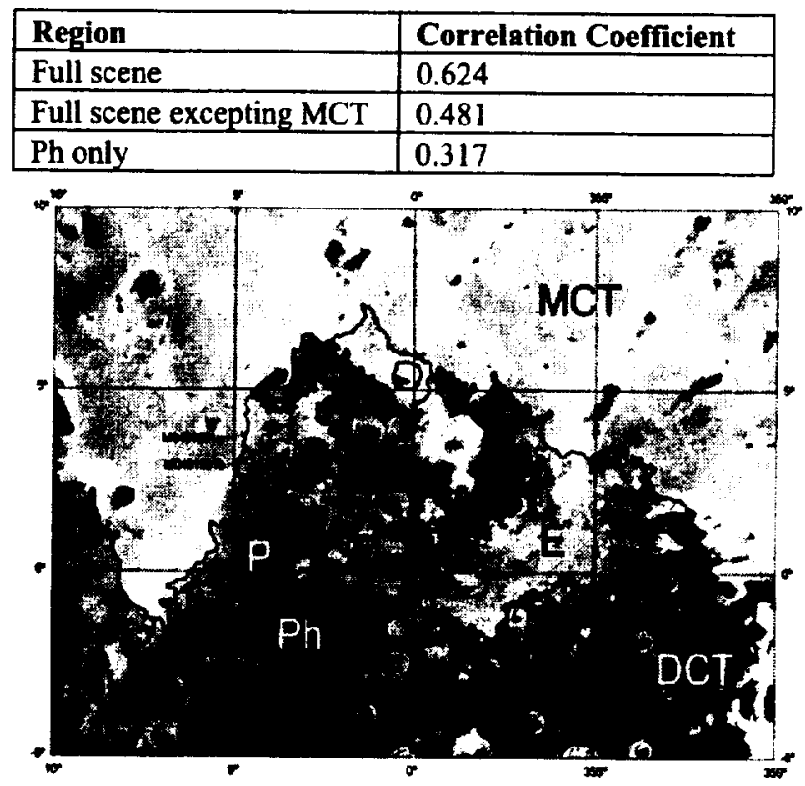

Figure 1. Units map for the Terra Meridiani study region. MCT: mantled cratered terrain; P: plains unit; Ph: hematite-bearing plains unit; E: etched unit; DCT: dissected cratered terrain.

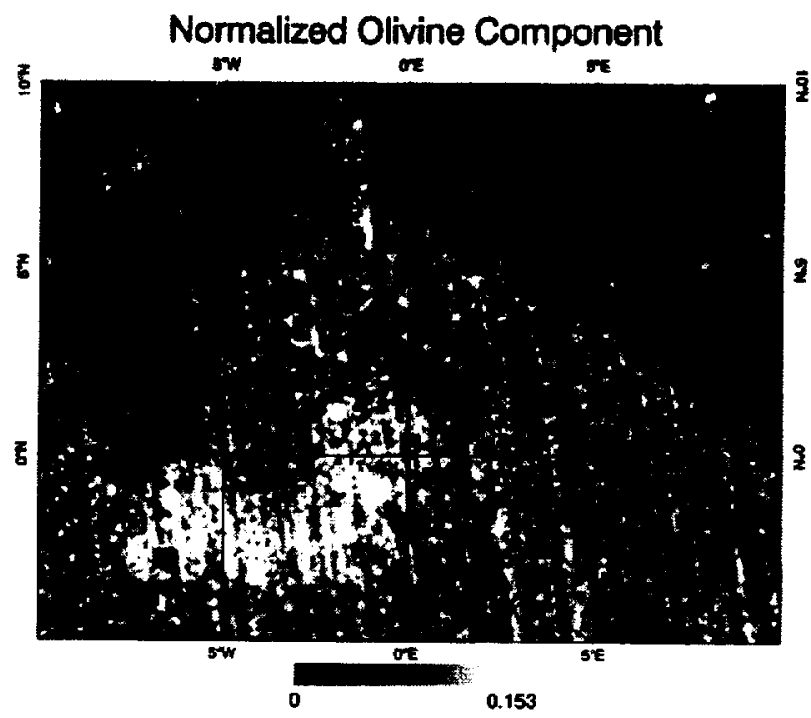

Figure 3. Normalized olivine component for the Terra Meridiani study region. 
INITLAL RESULTS FROM THE BLOOMSBURG UNIVERSITY GONIOMETER LABORATORY. M. K. Shepard, Dept. of Geography and Creosciences, 400 E. Second St., Bloomsburg University, Bloomsburg, PA 17815 (mshepard@bloomu.edu).

Introduction: The Bloomsburg University Goniometer Laboratory (B.U.G. Lab) consists of three systems for studying the photometric properties of sumples. The primary system is an automated goniometer capilble of measuring the entire bi-directional reflectance distribuion function (BRDF) of samples. Secondary systems in lude a reflectance spectrometer and digital video camera with macro zoom lens for characterizing and documenting othur physical properties of measured samples. Works complited or in progress include the characterization of the BRDF of calibration surfaces for the 2003 Mars Exploratiou Rovers (MER03), Martian analog soils including JSC-Mars-1, and tests of photometric models.

B.U.G. Lab: The B.U. gonionet:r is an automated 3axis system, providing for motion in incidence $\left(-60^{\circ}\right.$ to $+60^{\circ}$, emission $\left(-80^{\circ}\right.$ to $\left.+80^{\circ}\right)$, and azimuth angle $\left(0^{\circ}-180^{\circ}\right)$, thus allowing an entire half-hemisphere of icattering geometries to be measured (Fig. 1). Assuming the iample has a random (or at least azimuthally uniform) surface texture, the other half-hemisphere is the same by sy inmetry across the scattering plane. Maximum phase angle is $140^{\circ}$, minimum phase angle is $3^{\circ}$. Computer controlled stepper motors move all three axes with a precision of $\pm 0.001^{\circ}$. The light source is a $100 \mathrm{~W}$ quartz-tungsten halogen bulb and is powered by a radiometric-grade power supply. Thic output light is collimated, filtered with $50 \mathrm{~nm}$ FWHN interference filters, chopped, and fed to the incidence amı along a $1.3 \mathrm{~m}$ fiber optic bundle. Collimating optics at the top of the incidence arm provide a $2 \mathrm{~cm}$ spot on the sampl: from a distance of $56 \mathrm{~cm}$. The detector, sensitive to lighlt from $400 \mathrm{~nm}$ to $1000 \mathrm{~nm}$, consists of a silicon chip $1 \mathrm{~cm}^{2}$, amplifiers, and lock-in electronics that detect only the light chopped at the frequency and phase determined by the chopper on the source. It is located at the top of the emission arm a distance of $77 \mathrm{~cm}$ from the sample. The detector's field-of-view is restricted by a light shield giving an 1111 -vignetted FOV of $2.8^{\circ}$ and vignetted FOV of $25^{\circ}$. The sample dish is $57 \mathrm{~mm}$ in diameter and $10 \mathrm{~mm}$ deep, sits at the intersection of the three axes, and is stationary. Secondary icflections from the equipment surrounding the sample are minimized by flocking everything within the FOV of the detector. Control of the stepper motors and detector readout is completely automated and achieved using custom software written with LabView $^{\mathrm{TM}}$. Axes motion sequences are preprogrammed and saved. A typical run at a single wavelength measures the scattering behavior from $0^{\circ}$ to $60^{\circ}$ incicence $(l)$ in $15^{\circ}$ steps, emission (e) from $0^{\circ}$ to $80^{\circ}$ in $10^{\circ}$ stips, and azimuth $(a)$ from $0^{\circ}$ to $180^{\circ}$ in variable steps to thaintain a consistent solid angle between measurements of - 0.05 steradian. In all, each run of this type returns 680 data points and takes 82 minutes. Radiance factors are determined by dividing all sample radiances by that of a spectrahalon disk at $i=0^{\circ}, e=5^{\circ}$, and multiplying the result by $\cos (t) / \cos (e)$. The RMS precision of the 680 radiance factors are typically $\leq 1 \%$, while absolute accuracy is estimated to be $\leq 10 \%$.

Spectrometer and Digital Video Camera. A single grating spectrometer documents the reflectance spectrum (relative to spectrahalon) of samples from $400-1000 \mathrm{~nm}$ with a spectral resolution of $\sim 6 \mathrm{~nm}$. A Panasonic color video camera fitted with a macro-zoom lens is used to photographically document the samples. Typically two images are taken - one showing the entire sample dish (FOV $\sim 6 \mathrm{~cm}$, resolution of $\sim 100 \mu \mathrm{m}$ per pixel), the second zoomed in (FOV of $1 \mathrm{~cm}$, resolution of $\sim 15 \mu \mathrm{m}$ per pixel).

Examples: Figures 2a-c show an example of the data obtained by B.U.G. Lab on a sample of JSC Mars 1 analog soil provided by $\mathrm{E}$. Guinness. Fig $2 \mathrm{a}$ is a close-up of the soil, Fig $2 b$ is its spectrum, and Fig $2 c$ is a $3 D$ cylindrical plot of the scattering behavior observed for $F=45^{\circ}$ at $860 \mathrm{~nm}$. The $z-$ axis is in units of radiance factor. The radial axis is in units of emission angle, $0^{\circ}$ in the center to $80^{\circ}$ around the rim. Azimuth $\left(0^{\circ}\right)$ starts at the far right center. The scattering plane runs through the middle of the plot, forward scattering to the left, backscattering to the right. Note the overall backscattering behavior (a lambertian plot would be uniformly flat across the top), and the opposition surge.

Work Completed and in Progress: A number of projects have been completed or are underway.

Complete BRDF characterization of the calibration materials to be fown on the MERO3 rovers. Samples of material and the flight spare set of PANCAM filters were provided by J. Bell (Comell). Since the PANCAM is fixed relative to the calibration target on the rover, the emission angle was fixed at $53.5^{\circ}$ and incidence and azimuth were varied.

BRDF characterization of Martian analog soils. E. Guinness (Wash. U.) provided samples of JSC-Mars-1, and R. Morris (JSC) has provided four additional analogs. All of these samples were measured using the flight spare PANCAM filters and the data will be made available through the PDS after a brief analysis.

Controlled experiments to test photometric models. Well characterized particulate samples are being measured in a variety of particle sizes, packing densities, and surface roughness to test the ability of current photometric modeis to extract surface physical properties. 
Acknowledgements: The B.U.G. Laboratory was funded by a Major Equipment augmentation to the author's N.A.S.A. Planetary Geology and Geophysics grant. Current work is also funded through NASA PGG.

\section{Figure 1. Goniometer}

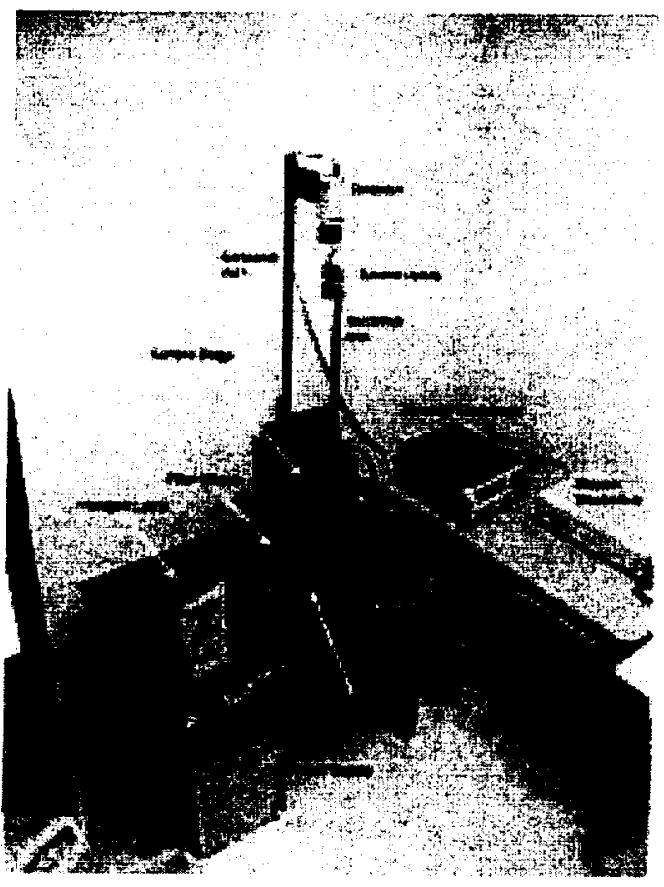

Figure 2a. Close-up of JSC Mars 1 (1 cm FOV)

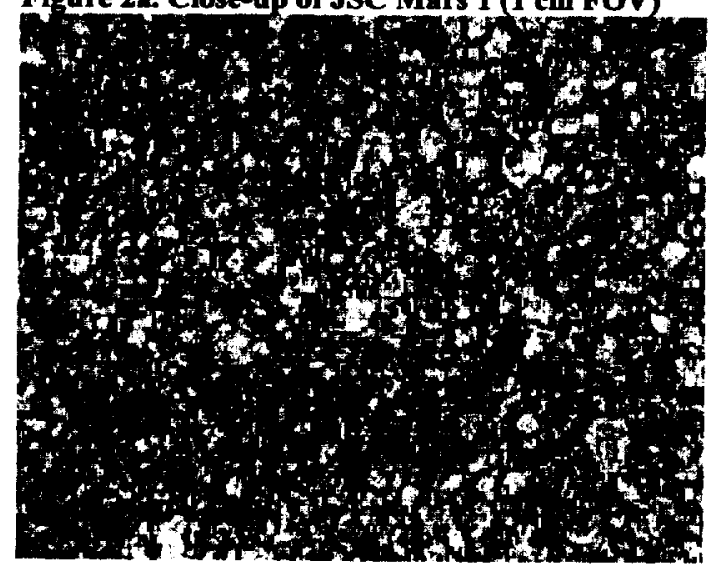

Figure 2b. Spectrum of JSC Mars 1

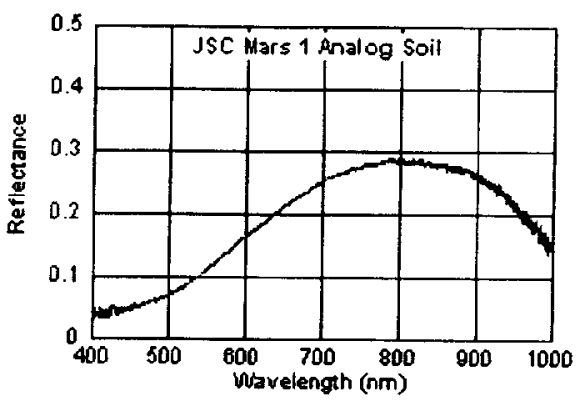

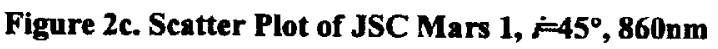

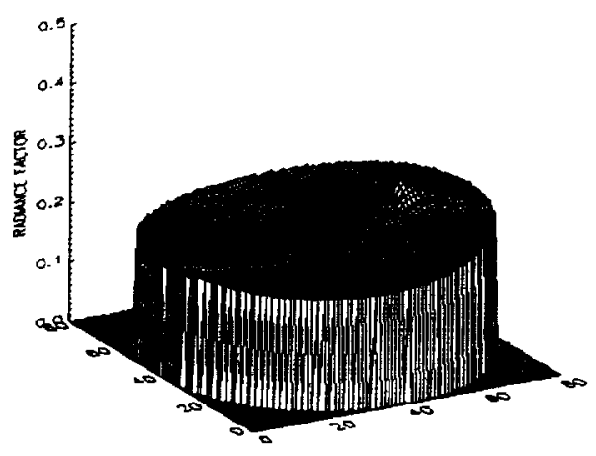


INTERPRETING PHOTOMETRY OF PLANETARY REGOLITHS: PROGRESS AND PROBLEMS AS SEEN FROM KHARKOV. Yu. (i. Shkuratov, Kharkov Astronomical Observatory, 35 Sumskaya St., Kharkov 61022, Ukraine, shkuratov@ygs.kharkov.ua

Introduction: Photometric observations of planetary regoliths demand a sophisticated interpreting basis that includes theoretical modeling, computer and laboratory simulations of light scattering. The high-level theoretical models were produced hy B. Hapke, e.g., [1] and they are widely used in planctology. At present time quick progress is observed in interpreting photometry. We overview here this progress and problems in understanding of physical mechanisms forming photometric properties of the planetary regoliths.

Physical mechanisms: The mechanisms important for interpreting photometry of the planetary regoliths are: shadowing (a) in particulate media and (b) on random topography, as well as (c) incoherent multiple scattering and (d) coherent backscatter enhancement.

(a) Shadowing in particulate media: This is the main effect contributing to photometric properties of the planetary regoliths at all phase angles even in cases when the regolith surfaces are not very absorbing. The shadow-hiding effect is studied vely well [1], nevertheless, a set of problems related to the effect remain unresolved. Recently, for instance, shadow-hiding effect was calculated for a particulate structure arranged as a fractal that is rather typical for the planetary surfaces [2]. This development seems to be progressive for interpreting photometry as it provides good fits for observations and operates with a new parameter, the number of hierarchical surface levels that is treated in the fractal manner as a fractional value. Note also that analytical calculations of the shadowing effect meet problems with densely packed particulate media. This is accounting for correlation in spatial distribution of the medium particles. The most effective way to overcome the problem is computer modeling, see, e.g., [3].

(b) Shadowing effect on random topography: At scales more than particle size, the planetary regoliths are complicated with random topography that also influences phase curves at large angles. A first approximation model accounting for the influence of random topography is presented in [1]. Improvements of this model are difficult, as the rigorous description of shadowing for single-valued random surfaces demands continual integrations that is hardly possible analytically [4]. Again the best way to calculate this effect is now a ray-tracing modeling [4]. The domain, corresponding to the scale of surface topography is comparable with size of surface particles, is studied very poor. Almost all theoretical models deal with largescale topographies described with graussian statistics, whereas so-called rocky topography being very useful for practice has not been studied at all.

A promising mathematically rigorous approach to calculate the influence of surface topography on photometric properties of the planetary surfaces can be applied for rather dark surface arranged hierarchically, when owing to large difference in scales a preceded hierarchical level of topography can be considered as a reference surface for the next topographical level [5]. This approach gives the following photometric disk function: $D(\alpha, b, l)=\cos [\pi(l-\alpha / 2) /(\pi-\alpha)](\cos b)^{\alpha /(\pi-\alpha)}, \alpha, l$, and $b$ being the phase angle, photometric longitude, and latitude, respectively. This formula describes very well the surface of the Moon and Mercury.

(c) Incoherent multiple scatter: If a regolith-like surface is weakly absorbing, multiple scattering can dominate photometric properties in the whole range of phase angles. The multiple scattering manifests itself inside and between particles as well as between elements of surface topography. Multiple scattering inside a particle can be considered when size of the particle is significantly larger that the wavelength. This forms the single-particle albedo and scattering indicatrix, which are often treated separately that is somewhat eclectic. Moreover, due to finite size of particles the shape of the indicatrix depends on the distance between the particles, if the distance is small enough. This is important for calculations of inter-particle multiple scattering.

To find phase curves for all orders of scattering, the radiative transfer equation is used. However this equation does not take into account correlation in ray propagation in media, whereas this correlation is important as it originates the shadowing effect. Our computer modeling shows that this factor is rather important even for higher scattering orders [6]. Another general problem of available theoretical models is treating particulate media as continual that is bounded with a plane, whereas real particulate media are discrete and have a transition upper layer, which is formed due to vertical fluctuations of particle number near the average level and can strongly influence the phase curves especially in the first order of scattering. This problem is effectively resolved by computer modeling $[3,6]$.

(d) Coherent backscatter enhancement is an important factor effecting at small phase angles on optical properties of regoliths even with low albedo. This enhancement is due to constructive interference of double passing light rays along the same trajectories in a par- 


\section{INTERPRETING PHOTOMETRY OF PLANETARY REGOLITHS: Yu. G. Shkuratov}

ticulate medium, e.g., $[1,2,7]$. The width of the coherent backscatter opposition spike depends on the characteristic distance between scatterers. For spikes with the width of a few degrees, the distance is of the order of 10 wavelengths. If the distance decreases the width increases. That is in principle the distance can be so small that the coherent backscatter effect will cover phase angles more than $20^{\circ}$. Nobody knows is it possible or not, as at small distance the ray approach should not work. Another question concerns the coherent backscatter effect for aggregated particles that are typical for the regoliths. What is the minimal size of the aggregates, for which the effect is still observable? This and many other questions have no answers yet.

There are several attempts to develop a combined model of the shadow-hiding and coherent backscatter effects, however, serious progress in this direction has not been yet achieved. The problem is that the scatterers of a medium should be considered simultaneously as large enough to cast shadows and small enough to form the coherent backscatter enhancement. A prospective way to develop this problem is to use two scales model when a medium is considered to consist of large enough $(\sim 100 \mu \mathrm{m})$ aggregates of small $(\sim 1$ $\mu \mathrm{m})$ graines. This allows one to consider separately the shadow-hiding effect (aggregate scale) and the coherent backscatter (grain scale) [2,7].

Laboratory simulations at small phase angles: Modeling photometric studies of many different samples, structural analogs of planetary regolith, with controlled parameters were recently carried out in Kharkov [7]. One Kharkov's photometer/polarimeter allows measurements at the phase angle range $\left(0.2-17.5^{\circ}\right)$. The measurements provide a basis to verify theoretical models. For instance it was found that for dielectric powders, both the opposition effect and negative polarization become more prominent when the samples are compressed. It was also found that the opposition effect and negative polarization parameters depend strongly on micro- and macro-structured optical inhomogeneities of scattering surfaces. These findings are not satisfactory explained by available theoretical models of the coherent backscatter enhancement. We plan to continue measurements paying attention to complicated samples that are made up by different materials and surface structures in order to model large scale inhomogeneity of planetary surface.

Geological aspects: A set of images acquired for the same scene of a planetary surface at different phase angles can be used to determine parameters of model photometric function, e.g., phase function slopes for each point within the scene. This allows one to use images of the surface in the photometric parameter for geologic interpretation. For example, phase function slopes for a few small lunar regions with Clementine UVVis images were mapped in [8]. The maps show several anomalous sites, e.g., small fresh impact craters have anomalous halos in these maps. The phase function within the halos is less steep than for surrounding mare surface. We interpret these halos to be due to geologically recent impact-induced alteration of the equilibrium millimeter-scale regolith structure. This structure is established due to micrometeorite bombardment at geologically short time scale. An anomaly of the same signature was found at the Apollo-15 landing site. We interpret it as a result of the regolith structure alteration with the lander jets. A unique photometric anomaly not correlated with albedo was found within the Reiner- $\gamma$ formation. This favors the impact hypothesis for the nature of the Reiner- $\gamma$ formation. Thus mapping the photometric characteristics is a new powerful tool in studies of the surface of atmosphereless celestial bodies, e.g., asteroids and Martian surface. This tool allows studies of recent seismic events and population of meteoroids in the inner Solar system. The ESA Smart-1 mission to the Moon will be a good opportunity to develop this direction, as during this mission many images of some regions of the lunar surface will be obtained at different phase angles.

Conclusion: The areas of interpreting photometry that seem to be prospective are:

(a) Accounting for the border effects in studies of the shadowing in particulate media;

(b) Consideration of fractal-like structures to model photometric properties of the planetary surfaces;

(c) Laboratory and computer simulations of photometric properties of micro- and macro-inhomogeneous particulate surfaces;

(d) Geological study of the images that present parameters of photometric function.

Acknowledgments. This studies was partially supported by INTAS grant \# 2000-0792.

References: [1] Hapke B. (1993) Reflectance and Emitance spectroscopy [2] Shkuratov Yu. and Helfenstein P. (2001) Icarus 152, 96-116. [3] Stankevich et al. (1999) JQSRT 63, 445-458. [4] Shkuratov Yu. et al. (2000) Thermal emission spectroscopy and analysis of dust, disk, and regoliths / Eds. M. Sitko et al. ASP (196). 221-230. [5] Shkuratov, Yu. (1995) Solar System Res., 29, 421-432. [6] Stankevich et al. (2002) $J Q S R T$ (in press). [7] Shkuratov Yu. et al. (1999) Icarus, 141, 132-155. [8] Kreslavsky M. et al (2000) JGR Planets, 105, 20,281-20,295. 
MERCURY'S SURFACE COMPOSITION FROM SURFACE SPECTROSCOPY. Ann L. Sprague, Lunar and Planetary Laboratory, University of Arizona, 1629 E. University Blvd. Tucson, AZ 85721 - 0092. sprague@Ipl.arizona.edu.

Introduction: During our 15 year quest to augment vis-near infrared spectroscopic $(0.4-2.5 \mu \mathrm{m})$ discoveries with mid-infrared $(2.5-13.5 \mu \mathrm{m})$ spectroscopic discoveries at Mercury, we have achieved much and learned even more. This talk (and paper) will describe some of those discoveries and lessons learned.

Methods used to interpret mid-infrared spectra include 1) identification of key spectral features diagnostic of composition. This has been achieved in the laboratory using terrestrial and lunar rocks, minerals, powders, and glasses. 2) comparison of laboratory and telescopic mid-infrared spectra of lunar soils from similar locations on the Moon. 3) use of the same spectrograph to obtain spectra of rocks, minerals, and powders to compare to spectra of the Moon's and Mercury's surface and thus calibrate spectrograph performance and resulting spectral character. 4) comparison of spectra obtained from spacecraft above all of the Earth's atmosphere of objects in the solar system (Jupiter, Saturn, asteroids) to those oltained from midinfrared instruments from ground-based observatories.

Near-infrared and mid-infrared diagnostics contrasted: The power of visible and near-infrared spectroscopy $(0.4-2.5 \mu \mathrm{m})$ has been demonstrated throughout the solar system for decades. For example, the size and shape of absorption bands in reflected light from the surface caused by crystal field transitions, metal-metal intervalence charge transfer transitions, and oxygen-metal charge transfer transitions of the materials in the regolith, are very diagnostic. The best known of these are probably the lunar olivine and pyroxene bands which permitted mapping of near-side abundance of these minerals and identification of the lunar mantle in Copernicus [1].

From $2.5-7 \mu \mathrm{m}$, volume scattering of light from regoliths becomes important. There are absorption (and emittance) features associated with photons scattering in individual grains. Many silicates, sulfates, and carbonates have diagnostic features in this spectral region. Most of this spectral region is available to spectrographs at high altitude at ground-based observatories and from stratospheric observatories like the Kuiper Airborne Observatory (KAO--retired) the Stratospheric Observatory for Infrared Astronomy (SOFIA--to be commissioned in 2005).

Major rock-forming minerals have their fundamental molecular vibration bands in the region from 7.5 $11 \mu \mathrm{m}$ (the Reststrahlen bands). The transparency feature between 11 and $13 \mu \mathrm{m}$ is associated with the change from surface to volume scattering. There are also major features associated with the bending, twisting modes of silicates, and other solar system materials occurring in the region from $13-40 \mu \mathrm{m}$.

An emissivity maximum (EM) associated with the principal Christiansen frequency, usually between 7 and $9 \mu \mathrm{m}$ is a good diagnostic of specific mineral identity and also bulk regolith or rock type in mixed mineralogic and textural assemblages common in regoliths.

Two good reviews for more details regarding the above highly-condensed and incomplete discussion above may be found in [2] and [3].

VIS/Near-infrared Discoveries at Mercury: Visible and near-infrared $(0.4-1.5 \mu \mathrm{m})$ spectroscopy has been successful in documenting the extremely low probability of presence of the $\mathrm{Fe}^{2+}$ charge-transfer absorption band in reflected light from Mercury's surface. This means that $\mathrm{FeO}$ in the regolith is very low in abundance, if present at all [4], [5], [6], [7]. In addition, the regolith, while mature, is more transparent than the regolith of the Moon, thus indicating low abundance of $\mathrm{Fe}$ blebs and other opaques that are characteristic of the lunar mature soils [8], [9].

Reflectance spectroscopy is useful on Mercury wherever there is reflected light. Of course spatial resolution is limited by the spectrograph aperture size. the plate scale of the telescope and detector, and Earth's atmospheric turbulance.

Mid-infrared Discoveries at Mercury: This spectral range is dominated by thermal emission. The location on the planet, for all data, is biased toward the hottest regions in the footprint of the spectrograph aperture. The other caveats of spatial resolution mentioned above for vis-near-ir observations also hold. In addition, spatial resolution is limited by the diffraction limit of the telescope.

Volume Scattering Region: Emissivity peaks at 5.7 and $6 \mu \mathrm{m}$, in a spectrum of Mercury from $100-160^{\circ}$ longitude [10], resemble prominent emissivity maxima at 5.7 and $6 \mu \mathrm{m}$ exhibited by low-iron olivine powders in laboratory spectra. The exact wavelength of the pair of peaks is seen, in laboratory studies, to shift by small amounts according to $\mathrm{FeO}$ content in the olivine.

A strong $5.5 \mu \mathrm{m}$ emission feature in a spectrum from $45-85^{\circ}$ longinde closely resembles that of laboratory clino-pyroxene powders [11]. The best fit is to diopside and the low-FeO abundance indicated by near-ir reflectance spectroscopy supports a low-iron bearing clino-pyroxene. Spectroscopic observations of 
Mercury have been made in this spectral region from the KAO [10], and between 3 and $7 \mu \mathrm{m}$ from $13,786 \mathrm{ft}$ altitude [11] at Mauna Kea (the NASA Infrared Telescope Facility, IRTF).

Emission Maximum in region of Principal Christiansen Frequency: Emissivity maxima (EM) at or close to $7.9-8.0 \mu \mathrm{m}$ and indicative of intermediate silica content $\left(\sim 50-57 \% \mathrm{SiO}_{2}\right)$ occur in spectra from $12-32^{\circ}$ and $22-44^{\circ}[12], 40-45^{\circ}[13], 45-85^{\circ}$ [11], $10-75^{\circ}[10]$, and $110-120^{\circ}$ [12] longitude. These locations fall in the inter crater plains east of the crater Homer. Spectra from $68-108^{\circ}[11]$ and $100-$ $160^{\circ}[10]$ longitude have multiple $\mathrm{EM}$ indicating a more complicated bulk composition and or mixed mineralogy of more basic composition $\left(45-49 \% \mathrm{SiO}_{2}\right)$.

Reststrahlen features: Reststrahlen features in spectra from $110-120^{\circ}$ longitude have been modeled with simple linear spectral mixing of laboratory spectra from $0-74 \mu \mathrm{m}$ powders. A mixture of labradorite (a mineral of the plagioclase feldspar solid solution with $\mathrm{Na}$-rich plagioclase, albite- $\mathrm{Ab}_{40}$ molar abundance) and low-iron ortho-pyroxene powders matches some but not all features in the Mercury spectrum. [14]. The Reststrahlen features in a spectrum from $68-108^{\circ}$ longitude have not yet been fitted with any model but show three emissivity peaks between 7.8 and $9.3 \mu \mathrm{m}$, some of which may be caused by Reststrahlen features. A composite spectrum from CVF spectral imaging at $205-240^{\circ}$ longitude [15] indicates a probability of picrite based upon comparison to a laboratory spectrum of one powdered picrite sample. In this case the sample was of a rock type with dominant low-iron olivine and other mineralogy.

Transparency minima: The Mercury spectrum from $110-120^{\circ}$ longitude has a clear and strong transparency minimum at $12.3 \mu \mathrm{m}$ that is at the same location as the transparency minimum in a laboratory spectrum of labradorite powders [12]. This is consistent with the location of the EM in the same spectrum as described above. Spectra from longitudes centered on $80^{\circ}, 256^{\circ}$ and $266^{\circ}$ have probable transparency minima at $12 \mu \mathrm{m} \mathrm{[16].} \mathrm{The} \mathrm{bulk} \mathrm{composition} \mathrm{associ-}$ ated with a transparency feature at this wavelength is intermediate to basic $\left(45-57 \% \mathrm{SiO}_{2}\right)$. Spectra from a region centered on $15^{\circ}$ has a minimum at $12.5 \mu \mathrm{m}$ [16] indicative of about $44 \% \mathrm{SiO}_{2}$ or an ultra-basic composition. A spectrum from a region centered on $229^{\circ}$ longitude has a doublet transparency minimum with one at $12.2 \mu \mathrm{m}$ and another at $12.5 \mu \mathrm{m}$. This is indicative of more complex mineralogy in that region.
Summary: About $40 \%$ of Mercury's surface has been measured spectroscopically. Roughly speaking the coverage is of the equatorial and low latitude regions at most, but not all, longitudes.

Mercury's surface composition is heterogeneous. Regions near Homer and the Murasaki Crater Complex appear feldspathic, trending toward $A b_{70}-A b_{40}$, more $\mathrm{Na}$-rich than the lunar anorthosites. Bulk compositions are of intermediate silica content. Some mixed compositions of more basic silica content are present in the regions from $68-160^{\circ}$ longitude but are not at all locations measured. Thus, it appears that low-iron basalts (mixtures of feldspar, pyroxene, and minor olivine) and $\mathrm{Na}$-rich feldspathic units dominate the intercrater plains.

Regions west of Caloris have mixed mineralogy and a more complex bulk composition with some basic and ultra-basic regolith types. There is evidence for picrite-like soils at $205-240^{\circ}$ longitude. According to transparency minima, an ultra-basic composition falls east of Homer crater near $15^{\circ}$. Two measurements indicate that ultra-basic regolith types are located far west of Caloris from about $205^{\circ}$ longitude perhaps to as far as $15^{\circ}$ longitude.

The picture above is painted in the broadest of strokes with the areal extent of the spatial footprint no smaller than $200 \mathrm{~km}$ by $200 \mathrm{~km}$ for the very best spatially resolved observations [12] and as much as 1000 $\mathrm{km}$ by $1000 \mathrm{~km}$ for the least spatially resolved [16]. More observations are called for.

References: [1] Pieters (1986) Rev. Geophys. 24, 557 - 578. [2] Pieters, C. M. and Englert, A. J. (1993), Remote Geochemical Analysis: Elemental and Mineralogical Composition, Cambridge University Press. [3] Cooper et al. (2002) JGR, 107, 10.1029/2000JE001462. [4] Vilas et al. (1986) Icarus, 59,60 - 68. [5] Blewett et al. (1997), lcarus, 129, 217 - 231. [6] Warell, J. (2002), Icarus 156, 303-317. [7] Warrel, J. (2002) Icarus, In Press. [8] Robinson, M. S. and Taylor, G. J. (2001) Meteoritics and Planetary Science 36, 841 - 847. [9] Mitchell, D. and dePater, I. (1994), Icarus, 110, 2 - 32. [10] Emery et al. (1998) Icarus, 136, 104 - 123. [11] Sprague et al. (2002) Meteoritics and Planetary Science In Press. [12] Sprague et al. (1994) Icarus 109, 156 - 167. [13] Tyler et al. (1988) GRL, 15, 808 - 811. [14] Sprague, A. L. and Roush, T. L. (2000) lcarus, 133, $174-183$. [15] Sprague et al. (2000), lcarus, 147, 421 - 432. [16] Cooper et al. (2001) JGR 106, 32803-32814. 
LIGHT SCATTER BY PARTICULATE SURFACES WITH DIFFERENT TOPOGRAPHY. D. Stankevich ${ }^{1}$, Yu. Shkuratov', P. Pinet', and A. Cord ${ }^{2}$. 'Kharkov Astronomical Observatory (35 Sumskaya St., Kharkov 61022, Ukraine. Stankevich@astron.kharkov.ua). ${ }^{2}$ Observatoire Midi-Pyrénées (14 Av. E. Belin, 31400 Toulouse, France).

Introduction: Significant increase of the spatial resolution of optical experiments on and near the planetary surfaces, e.g., in-situ measurements for Mars Pathfinder mission and soon-to-come orbital high resolution multispectral observation; for Mars Express mission, demands efforts in laboratory and theoretical simulations of imaging spectrophotometric studies to interpret physical and mineralogical characteristics of the planetary surfaces. For this reaion a new spectral imaging facility has been designed and settled since April 2000 at the Observatory Midi-Pyrenees [1]. This is intended to help the definition of new spaceborne instruments, to simulate planetary observations, and to improve the understanding of the effects of observational conditions and of physical properties on the bidirectional reflectance of rocky surfaces and soils. Attempts to interpret laboratory measurements carried out with the facility using the standard photometric model by Hapke [2]. Sometimes it may result in non-unique solution with ambiguous physical interpretation $[3,6]$. Hapke's model implicitly consider; that the scale of surface topography is much larger than the size of regolith particles handled as a particulate medium. Besides, this topography is described with the Gaussian statistics. However, in laboratory and in-situ planetary measurements one deals with surface topographies that have the characteristic scale comparable with the size of regolith particles, and often such meso-scale topographies are formed by rock fragments $[1,3,6,7]$ (see in this volume an abstract by Cord et al.). To study this effect, we carried out a computer modeling of multiple light scattering in particulate media complicated by rocky topographies and by a single-valued random surface with the characteristic scale of order of particle size. The geometric optics approximation is used.

Computer model for particulate media and different surface topography. We produce a new computer model for calculations of multiple scattering in arbitrary systems of opaque diffusely scattering particles. Besides, we carry out simulations of the shadowhiding effect for surfaces with different topographies, using this in combination with the model for particulate media. Our computer experiments simulate what happens in nature or laboratory studies: a system of particles generated in a computer memory is "illuminated" by rays, which are traced in the system from the light source to the observer. Unlike our previous models $[4,5]$ the model used here is able to simulate effectively multiple scattering in semi-infinite particulate media with arbitrary surface topographies having the charac- teristic scale comparable with the size of medium particles. For the sake of simplicity the particles are supposed to be spherical with the same size and Lambertian surface. We use albedo particle surface equal 0.8 . This allows us to restrict calculations by the first 5 scattering orders. As in previous studies [4,5], our computer modeling consists of two separate stages: (1) generation of a random system of particles with certain volume density $\rho$ and (2) Monte Carlo ray tracing. We use a system of particles randomly distributed in a rectangular parallelepiped. The upper border of this parallelepiped (the model frame) can be undulated (or complicated) by a topography. The lateral and bottom planes are considered to be cyclically closed. That is, if a ray leaves the parallelepiped volume, e.g., through the lateral plane, it comes into the same volume from the opposite side. This is an effective way to simulate a semi-infinite medium with a finite volume.

Results and discussion: Although our model allows calculations at arbitrary illumination/observation geometry we focused here on two of them. We use the "mirror" geometry, $i=\varepsilon=\alpha / 2, \delta=\pi$, where $i$ and $\varepsilon$ are the angles of incidence and emergence, respectively, $\delta$ is the azimuthal angle, and $\alpha$ is the phase angle. Besides we use an azimuthal geometry at $i=\varepsilon=85^{\circ}, \delta \approx$ $\alpha$. We compare three border topographies: (a) flat-inaverage border of a particulate medium; (b) border a particulate medium undulated by a random topography with the Gaussian distribution of heights and Gaussian correlation function; (c) border of a particulate medium complicated by a thin layer of "rocks" randomly distributed (with random embedding) over the border of the fine-grained particulate medium.

Before to study media with complicated borders we model the shadow-hiding effect for a Gaussian (G) topography with RMS slope equals 1 and for rocky topography formed with spherical stones randomly embedded in a substrate with the flat boundary (Fig. 1). Thus the latter is formed by the boundary and the surface of stones laid upper the boundary. The volume density of stone layer is taken 0.3 . This density provides almost the same number of details in the model frame as in case of G-topography.

Fig. I shows phase curves of the probability that a ray is not interrupted by G- and rocky (R-) topographies at both the mirror $(-M)$ and azimuthal $(-A)$ geometries. As one can see at the M-geometry the Rtopography shows much steeper curve at small phase angles than in case of G-relief. This is quite reasonable 
LIGHT SCATTER BY PARTICULATE SURFACES... D. G. Stankevich et al.

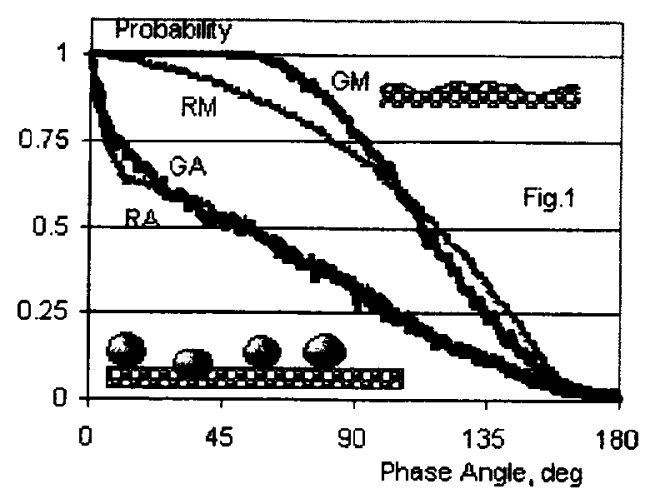

as R-topographies have angles of surface slopes greater than $90^{\circ}$. While at the A-geometry the shadow-hiding probability for these so different types of topography reveals very similar phase angle behaviors.

Calculations of phase-angle curves for semi-infinite media (with $\rho_{\text {medium }}=0.1$ ), whose borders are flat-inaverage (F) and complicated by $G-$ and $R$ - (at $\rho_{\text {stones }}=$ $0.3)$ topographies, are presented in Figs. 2 and 3 for the A- and M-geometries, respectively. The main parameter of G-topography is the RMS slope that is equal to the ratio of RMS heights deviation and horizontal correlation radius $L$. Another important parameter is the mean size of topography details, which is about $L$. We used $L=8 r$, where $r$ is the medium particle radius. As a characteristics of $\mathrm{R}$-relief we use the radius of "stones" $R=10 r$. These values are chosen to obtain the shadowing properties of R-topography maximally close to G-one. We consider also that the thickness of the stone layer equals $2 R$, the stone surface is characterized by Lambertian indicatrix, and multiple scattering taken place for R-topography and in the basic medium.

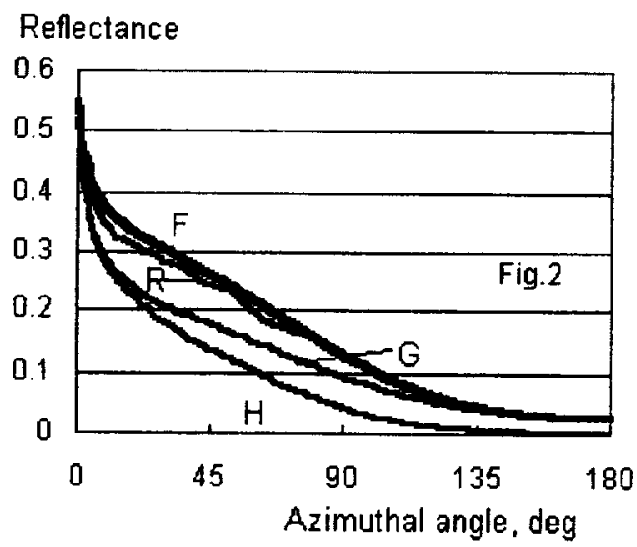

Figs. 2 and 3 clearly show the opposition effect. The brightness surge seen in Fig. 2 is related to correlations between incident and emergent rays in both the particulate medium and the surface topography at small phase angles. We note that for M-geometry (Fig. 3) curves $F$ and $G$ are almost coincident. They go separately in the A-geometry (Fig. 2), though at large phase angles they tend to be close to each other. This closeness of the $F$ and $G$ curves is due to that details of the G-topography consist of too small numbers of medium particles: the details are transparent enough at large phase angles in both the $\mathrm{A}$ - and $\mathrm{M}$-geometries. At small phase angles the G-topography can affect results only at A-geometry (as it should be). For comparison Figs. 2 and 3 show curves $\mathrm{H}$ obtained by multiplication of curves $\mathrm{F}$ by corresponding shadow-hiding probabilities shown in Fig. 2. Such a factorized form is used, when details of topography are not transparent, i.e. when $L \gg r$ [2]. As one can see at large phase angles this factorization gives results that are very distinct from the cases when multiple scattering between topography elements is taken into account. Curves $\mathrm{R}$ in Fig. 2 and 3 show that the surface particulate topography noticeably influences on the photometric properties.

Conclusion: We showed that particulate media with borders complicated by G- and R-topographies reveal different photometric behavior in comparison with the case of the factorized approximation usually used in theoretical models. This points at the need for an improved theoretical modeling for describing the real case of natural regolithic surfaces, for which the meso-scale organization of the textural relief influences the surface photometric behavior.

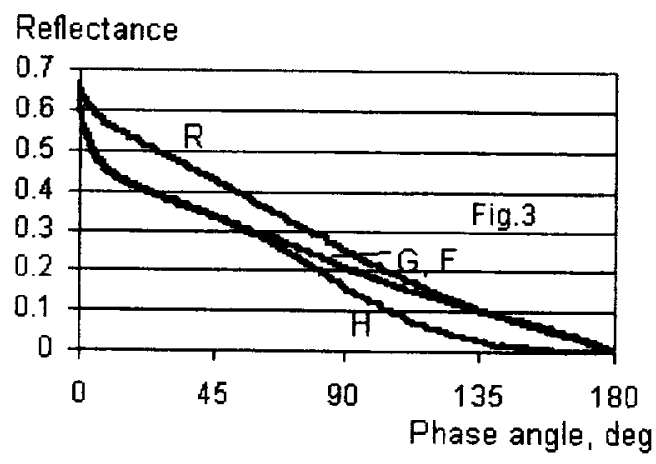

Acknowledgement. This work is partially supported by INTAS grant \# 2000-0792.

References: [1] Pinet P. et al. (2001) LPSC XXXII, Abstract 1559. [2] Hapke B. (1993) Theory of reflectance and emittance spectroscopy. Cambridge Univ. Press. 450 p. [3] Cord A. et al. (2002) lcarus (submitted). [4] Stankevich D., et al. (1999) JQSRT, 63, 445458. [5] Stankevich D., et al. (2000) Optics \& Spectroscopy, 88. 682-685. [6] Cord A., et al. (2002) 27-th EGS Proc. PS2 session, p. 268. [7] Cord A., et al. (2002) 27th EGS Proc. PS4 session, p. 271. 
COMBINING THE MGM AND HAPKE THEORY: TWO MODELS ARE BETTER THAN ONE. J. M. Sunshine' ${ }^{1}$, B. E. Clark', P. G. Lucey' 'Advanced Technology Applications Division, Science Applications International Corporation (SAIC), Suite 400, 4051 Daly Dr., Chantilly, VA 20151 (sunshinej@saic.com), ${ }^{{ }^{2}}$ hysics Department, Ithaca College, Ithaca, NY 14850 (bclark@ithaca.edu) ${ }^{3}$ Hawaii Institute of Geophysics and Planetology, University of Hawaii at Manoa, 2525 Correa Rd., Honolulu HI 96822 (lucey@pgd.hawaii.edu).

Introduction: Over the last several decades, remote spectroscopic studies have very successfully been used to explore the composition of various solid surfaces in the solar system. Building on these initial successes with mineral identification, recent efforts have focused on more detailed spectral inicrences including assessing the composition and abundance of mafic minerals. Such efforts are complicated by the fact that most silicate-rich surfaces include lithologic combinations of several mafic minerals which have overlapping absorption bands.

Two of the more quantitative app w uaches to spectral interpretation are non-linear mixture: modeling based on Hapke's radiative transfer theory [1] and absorption band modeling [2]. Both have been used successfully, yet as described below, these two methods are very complimentary offer even greater promise if they can be successfully combined.

Absorption Band Modeling: The Modified Gaussian Model (MGM,[2]) of absorption bands was developed to specifically resolve overlapping absorption bands in spectra. Under the MGM. spectra are modeled as a sum of absorption bands (eich represented by a Modified Gaussian distribution) superimposed onto a baseline continuum. The shape of these distributions was derived theoretically, and emfirically shown to accurately model isolated absorption bands. Each absorption band is characterized by a center, width, and strength, and the continuum is descriled by a slope and offset (all as function of energy). Using a simple BeerLambert approximation for absorbance (e.g., "apparent absorbance" [3]), modeling takes place as a function of $\log$ reflectance and energy. The MC $\mathrm{MM}$ has been used to characterize continuous changes in individual absortpions as a function of compositisn in several common mafic minerals including olivine and pyroxenes and successfully used to establish constraints on the composition of asteroidal, martian, and lunar surfaces.

Non-Linear (Hapke) Mixture Modeling: Absorption band modeling has many strengths, yet one of its drawbacks is that is does not account for albedo. In contrast, results from mixture models specifically rely on the albedo of various minerals. In addition to albedo (and single-scattering albedo in particular), the Hapke model combines mineral spectra based on radiative transfer equations that also include surface roughness and average single particle phaie functions. This mixture framework allows the addition of mineral phases (metal and other opaques) that do not have specific absorption features that can be modeled with the MGM. In addition, the effects of space weathering can be explicitly included within the framework of Hapke mixture models [Hapke, 2001].

Mixture models also have disadvantages, including the fact that they are very dependent on the choice of endmember spectral components [4]. Typically, laboratory analogs (terrestrial or metcoritic samples) are used as endmembers in modeling remote spectra. Recently these "library" materials have also be augmented with spectra calculated from optical constants $[5,6]$. However, it is difficult to find endmembers that have the same major element, minor element, and physical state (grain size, temperature, texture) as those observed on a remote surface.

Integration of the Two Methods: Many of these issues in mixture modeling can be overcome by using information derived from MGM absorption band modeling, which derives information directly from the actual spectrum as measured. Absorption band and Hapke mixture models are therefore quite complimentary. In particular, results from MGM on mineral compositions can be used to constrain endmember compositions and modal abundances for use in Hapke-based mixture models. These Hapke models in-turn will provide better estimates of abundance than the MGM alone.

In addition, many of the first order approximations within the MGM, including a polynomial continuum (representing first surface reflectance) and its simple absorbance model, can clearly be improved with Hapke theory.

We will explore various approaches to integrating these two model and assess their utility by applying them to laboratory spectra of controlled mineral mixtures, well characterized natural samples (e.g., meteorites), and space-altered surfaces (the moon and silicaterich asteroids). By combining the MGM with Hapke theory we hope to improve our ability to remotely constrain mineralogy from reflectance spectra.

\section{References:}

[1] B. Hapke, Theory of Reflectance and Emittance Spectroscopy, 1993.

[2] J. Sunshine, C. Pieters, and S. Pratt, J.G.R., 1990.

[3] R. Clark and T. Roush, J.G.R., 1984.

[4] B. Clark, J.G.R., 1995.

[5] B. Clark et al., MAPS, 2001.

[6] P. Lucey, J.G.R., 1998. 



\section{ORIGIN OF SURFACE-CORRELATED AND AGGLUTINITIC NANOPHASE FE" A Bedtime Story for Bruce. \\ Lawrence A. Taylor (lataylor (wi) utk.edu); Planetary Geosciences Institute, Department of Geological Sciences, Universily of Tennessee, Knoxville, TN 37996}

Introduction: It has become accepted lore that the myriad of grains of nanophase $\mathrm{Fe}^{0}$ (abbrev. npFe ${ }^{0}$ ) in lunar agglutinates are the result of "auto-reduction of impact-melted lunar soil in the presence of solar-wind hydrogen" [1]. However, recent studies have demonstrated other sources of $\mathrm{npFe}^{0}$ in lunar soils [2-3], as present in thin patinas $(\sim 0.1 \mu \mathrm{m})$ on the surfaces of most soil particles [4]. The major portion of this $\mathrm{npFe}^{0}$ formed by deposition of vapor produced by abundant micrometeorite impacts, as documented by the presence of multiple and overlapping patinas. A smaller portion may have formed by radiation sputtering [5]. The vapor deposition of this patena npFe ${ }^{0}$ is not actually at the surface, sensu stricto, but just below [3]. It would appear that the presence of $n p F e^{0}$ in the vapor-deposited patinas (rims) on virtually all grains of a mature soil [G] provides an additional and abundant source for the greatly increased $1_{\mathrm{s}} / \mathrm{FeO}$ values observed [7]. [At this point, I would like to see just how red with fury Bruce's face must be, as the above statements appear to steal his "I told you so's.".] You see, it was Bruce and his Pittsburgh colleagues that had astutely predicted all this way back in the Apollo days, but its significance went largely overlooked for decades. For an elegant and thorough review, albeit with a bit of bias, please read Hapke [8].

\section{Agglutinates by Auto-reduction of $\mathrm{FeO}$ :} The reaction that supposedly occurs involves in the auto-reduction paradigm involves the presence of solar-wind protons during impact-melting of lunar soil -$\mathrm{FeO}_{(\mathrm{l})}+2 \mathrm{H}^{+}=\mathrm{Fe}^{0}=\mathrm{H}_{2} \mathrm{O}$. Obviously much of the water was lost to space, but with the fine-grain size of the resultant $n p-$ $\mathrm{Fe}^{0}$ intimately intergrown and dispersed in the glass, one would suspect that there should be distinct remnants of the water trapped within the glass. Yet, detailed FTIR studies by Taylor et al. [9] failed to detect any traces ( $>5 \mathrm{ppm}$ ) of water in numerous agglutinitic glasses. This fact alone should make one suspicious of the hydrogen autoreduction theory.

Surface-Correlated npFe ${ }^{0}:$ The Lunar Soil Characterization Consortium [e.g., 10] was established to coordinate detailed study of the physical and chemical properties of the finest fractions of numerous lunar soils $(<45-20,20-10$, and $<10 \mu \mathrm{m})$. They have produced hard data and have come to the following conclusions: 1) the abundances of agglutinitic glass and the $I_{s} / F e O$ values increase with decreasing grain size; 2) if the increase in $I_{S} / F e O$ that is attributable to the increase in agglutinitic glass is accounted for in each change in grain size, the "residual" is the possible surface-correlated $I_{s} / \mathrm{FeO}$ contribution; and 3) on average, there is about a $100 \%$ increase in $I_{s} / \mathrm{FeO}$ value between the finest two size fractions, with only about $10 \%$ increase in agglutinitic glass. These general large increases in $1_{s} / \mathrm{FeO}$ versus nominal increases in agglutinitic glass contents are also demonstrated in the $<10 \mu \mathrm{m}$ fraction of the mare soils, as shown below [7]. 
TABLE 1. Characteristics of Mare Soils.

\section{Low-Ti Soils High-Ti Soils}

$\begin{array}{rlrrr}\mathbf{I}_{S} / \mathrm{FeO}_{(250 \mu \mathrm{m})} & 14 & 94 & 14 & 81 \\ \begin{array}{c}(\text { Maturity) } \\ \text { I }_{s} / \mathrm{FeO}_{(<10 \mu \mathrm{m})}\end{array} & 35 & 160 & 30 & 160 \\ \text { Agglut. Glass } & 55 \% & 70 & 45 & 62\end{array}$

Origin of Agglutinitic Glass: It has been demonstrated conclusively by the research of the LSCC [7] that it is the fusion of the finest fraction $\left(\mathrm{F}^{3}\right.$ model; 11] of lunar soil that forms the majority of the agglutinitic glass. But, it is exactly this $<10 \mu \mathrm{m}$ fraction that also contains the vast majority of the surface-correlated $n p F e^{0}$. It is considered entirely possible that most of the $\mathrm{npFe}^{0}$ in the "agglutinitic glass" had its ultimate origin as vapor-deposited $\mathrm{npFe}^{\mathbf{0}}$ in patinas on soil particles, which were subsequently melted and became part of the glass component. Effectively, as the $<10$ um fraction contains the most vapor-deposited $\mathrm{npFe}{ }^{0}$, this is melted and incorporated into impact glass. The average size of the $\mathrm{npFe}^{0}$ in this recycled glass (agglutinate) increases. This correlates with the data of Keller et al. [12] and James et al [13], where the average grain size $n \mathrm{pFe}^{0}$ in "agglutinitic glass" is larger than in vapor-deposited $\mathrm{npFe}$.

New Minerals: Another significance of the process of vapor-deposition of $n \mathrm{pFe}^{0}$ was recently discovered in a regolith breccia clast in lunar meteorite Dhofar 280 [14]. This clast contains numerous, dispersed micron size $(0.5-10 \mu \mathrm{m}) \mathrm{FeNi}$ metal grains, similar to an agglutinate-glass rich soil. In addition, grains of three new $\mathrm{Fe}-\mathrm{Si}$ phases are present that vary in size from $2-30 \mu \mathrm{m}$. We have reported, for the first time, the occurrence of iron-silicides in lunar rocks.
These Fe-Si compounds have approximate compositions of: $\mathrm{FeSi}, \mathrm{Fe}_{2} \mathrm{Si}$ and $\mathrm{FeSi}_{2}$.

A likely possible scenario for the formation of these phases involves the melting and vaporization of lunar soil by micro-meteorite impact. It is thought that the ultimate origin of most of this $\mathrm{npFe}^{0}$ is from the vaporization of $\mathrm{FeO}$ and $\mathrm{SiO}_{2}$ from the melting of the soil. The $\mathrm{FeO}$ dissociates to $\mathrm{Fe}$ and $\mathrm{O}$ and the $\mathrm{Fe}$ is condensed onto the soil particles. However, no consideration of the $\mathrm{SiO}_{2}$ in the vapor-phase has ever been made. We suggest that the $\mathrm{SiO}_{2}$ in the vapor also dissociates to $\mathrm{SiO}$ and $\mathrm{Si}$. It is thermodynamically possible that the $\mathrm{Fe}$ in the vapor combined with various proportions of $\mathrm{Si}$, also in the vapor and was condensed as small Fe-Si grains, in a crude way similar to the condensation of CalciumAluminum-rich Inclusions (CAIs). If these $\mathrm{Fe}-\mathrm{Si}$ phases are the result of impactproduced vaporization of lunar soil components, it is predicted that many of the small, micron-sized $\mathrm{Fe}$ metal grains that occur in the lunar soil may well contain $\mathrm{Si}$ and $\mathrm{Fe}$-Si phases as well.

References: [1] Housley et al., 1973, PLSC 4, 2737-2739; [2] Keller and McKay, 1993, Science 261, 1305; [3] Keller and McKay, 1997, GCA 61, 2331; [4] Wentworth et al., 1999, MaPS 34, 593. [5] Bematowicz et al., 1994, LPSC XXV, 105; [6] Keller et al., 1999, New Views II; [7] Taylor et al., 2001, MaPS 36, 285; [8] Hapke, B., 2001, JGRPlanets 106, 10,039; [9] Taylor et al., 1995, LPSC XXVI, 1399; [10] Taylor et al., 2001b, JGR-P 106,27985; [11] Papike et al., 1981, PLPSC12, 409; [12] Keller et al., 2000, LPSC XXXI, LPI CD-ROM \#1655; [13] James et al., 2002, LPSC XXXIII, LPI CD-ROM \#1655; [14] Anand et al., 2002, LPSC XXXIII, LPI CD-ROM \#1653. 
UNMIXING THE LUNAR SURFACE. S. Tompkins, SAIC, 4501 Daly Drive, Suite 400, Chantilly, VA 20151-3707 (tompkinss@saic.com).

Introduction: Spectral mixture inalysis has been shown to be a powerful, multifaceted tool for the analysis of spectral image data [e.g., I, 2]. In this approach small areas thought to represent distinct lithologies are chosen within the image. The spectral properties of these representative "endmembers" are then combined in a least-squares mixing algorithm to provide the best fit for each pixel within the image. Each pixel is thus represented by the prisportion of each endmember required to minimize the difference between the model and the actual spectra. Applications to both lunar and terrestrial remote sensing include mapping soils and exposed bedrock and modeling surface processes [e.g., 3, 4].

Linear vs. Non-Linear Mixing: Depending on the application, spectral image data can be "unmixed" assuming linear mixing [5], or non-line $: 1$ mixing $[6,7]$. Under the former, the reflectance specirum of a mixture is assumed to be a linear combination of the endmember reflectance spectra. Non-line ar mixing, on the other hand, is more appropriate in multiple scattering regimes, and each endmember's spectral contribution to the mixture spectrum is weighted by the endmember's optical extinction coeffient [7]. It has been demonstrated that a non-linear mixing model more accurately predicts abundances for the Moun's surface particularly of low albedo materials - and is more appropriate for most lunar science questions [8].

Examples: Recent examples of lunar applications of mixture analysis have focused on: (a) merging high spectral resolution data with high spatial resolution imagery [9] (Figure 1), (b) on estimat Ing and removing the contribution of shade/shadow from a scene to allow more accurate compositional estimates: [10] (Figure 2), and (c) on estimating and modeling sill transport across mare-highland boundaries $[8,11]$. In the data merging example, a linear mixing model has been applied with promising results; future work, however, will include nonlinear mixing as part of the data modeling. With shade removal, both linear and non-linear models have been applied. The non-linear model appears to better separate low-albedo materials from shade, although further validation is needed to assess the results more fully. Finally, in mojeling marehighland mixing [11], a non-linear $m$, del was used to estimate the amount of mare and highland soils distributed across major contacts, and to de velop a physical model for transport.
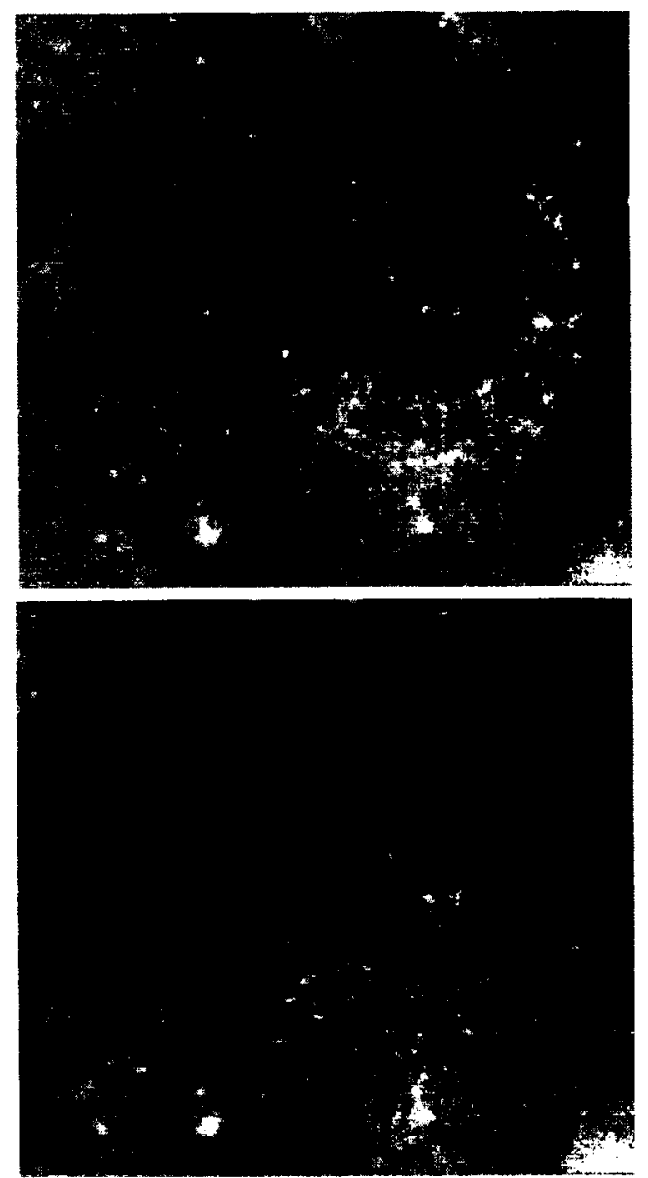

Figure 1: From [9] Top: Mafic $.75 \mu \mathrm{m} / 95 \mu \mathrm{m}$ ratio (a widely used multispectral surrogate for $1 \mu \mathrm{m}$ band depth) of Copernicus crater, using Clementine UVVIS camera data. Bottom: $1 \mu \mathrm{m}$ band depth image derived from calculated NIR data (created by combining multispectral data and telescopic NIR spectra for the same locations on the Moon via a mixture model). Albedo artifacts are eliminated in the band depth image. 

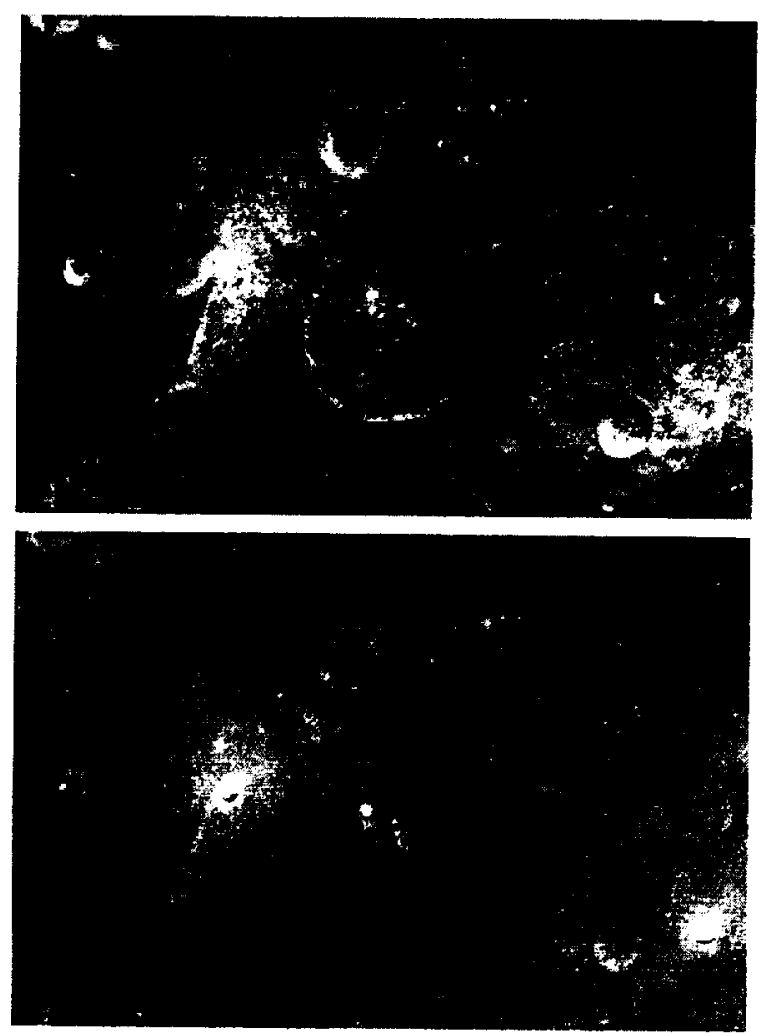

Figure 2: Top: Original Clementine UVVIS camera image at $0.75 \mu \mathrm{m}$ of Stevinus Crater. Note shadows within craters. Bottom: The same image after shade removal using a mixing model. Black spots in small fresh craters are pixels that are saturated in the original calibrated data.

In transforming remote sensing data into quantitative physical parameters, linear spectral mixture analysis has proven to be both diverse in its applicability and robust. The addition of Hapke's photometric model to allow more realistic separation of intimate mixtures has served to make it an even more powerful tool, to which we currently owe a portion of our understanding of lunar surface composition and evolution.

\section{References:}

1. Adams, J.B., et al., J. G. R., 1986. 91: 8113-8125.

2. Smith, M.O., et al., Rem. Sens. Environment, 1990. 31: $1-26$.

3. Roberts, D.A., et al., Rem. Sens. Environment, 1993. 44: $255-269$.

4. Mustard, J.F., et al., J. G. R., 1993. 98: 3387-3400.

5. Adams, J.B., et al., in Remote Geochemical Analyses: Elemental and Mineralogical Composition, 145166.

6. Hapke, B., Theory of reflectance and emittance spectroscopy. 455.

7. Mustard, J.F. and C.M. Pieters, J. G. R., 1989. 94.
8. Mustard, J.F., et al., J. G. R., 1998. 103: 19,41919,425 .

9. Sunshine, J.M. and S. Tompkins, LPSC XXXII, 2001.

10. Tompkins, S., LPSC XXXIII, 2002.

11. Li, L. and J.F. Mustard, J. G. R., 2000. 105(E8): 20,341-20,450. 
Hubble Space Telescope Photometry of Mimas, Enceladus, Tethys, Dione, and Rhea. A. Verbiscer $^{1}$, R. French ${ }^{2}$, and C. McGhee ${ }^{2},{ }^{1}$ Department of Astronomy, University of Virginia, P.O. Box 400325, 530 McCormick Road, Charlottesville, VA 22903, verbiscer@virginia.edu, ${ }^{2}$ Department of Astronomy, Wellesley College, Wellesley MA 02181, rfrench@wellesley.edu, cmghee@firstclass.wellesley.edu

The innermost of Saturn's classical satellites, Mimas and Enceladus, have historically proven challenging observing targets for Eartl]-based telescopes due to their proximity to the bright planet and rings. Since the ring plane crossings in 1995 and 1996, in preparation for the upcoming Cassini mission in 2004, the Hubble Space Telescope's Wicle-Field Planetary Camera (WFPC2) has been monitoring the Saturn system at each opposition as well as near quadrature each year (R. French, PI). This observing program has provided numerous images of Saturn's classical satellites in each of the five WFPC2 wideband UVBRI filters (F336W, F439W, F:55W, F675W, and F814W) and occasionally in the F255W, F785LP, and F1042M filters at solar phase angles between $0.26^{\circ}$ and $6.4^{\circ}$.

We have produced mutually consistent solar phase and rotation curves for Mimas, Finceladus, Tethys, Dione, and Rhea and report the first observation of the steep, narrow opposition effects of Mimas and Enceladus at visible wavelengths, necessitating an increase in currently accepted value's of their geometric albedos. Among these satellites, only Rhea was known to have a strong opposition effect in the visible [1], and 1995 ring plane crossing observations revealed Enceladus' large opposition surge in the near-infrared [2]. Combining thes: new observations with existing Voyager and telescopic data enables a more precise determination of the physical characteristics of regolith particles comprising the surfaces of these satellites. Application of the Hapke photometric equation [3] to phase curves which include near opposition data yields information on the directional scattering behavior of surface particles, regolith compaction, and the degree to which coherent backscatter plays a role in the scattering of light from the particulate surface of an icy satellite: [4]. Comparisons between the derived photometric parameters for each satellite suggest the degree to which location - either interior (Mimas and Enceladus) or exterior (Tethys, Dione, and Rhea) to the densest point in the E-ring
- affects the physical properties of regolith particles. Mimas and Enceladus have brighter trailing hemispheres [5-8], while Tethys, Dione, and Rhea display the opposite hemispherical albedo dichotomy $[5,8$, 9].

Multiple observations during a single HST orbit provide a series of measurements of the brightness of a satellite at constant phase angle, longitude, and latitude in each of the five filters, UVBRI, occasionally spanning a range from $255-1042 \mathrm{~nm}$. These spectral data indicate the satellite's color and demonstrate wavelength dependence on albedo.

References: [1] Domingue et al., 1995, Icarus 115, 228-249. [2] Momary et al., 2000, Icarus 148, 397406. [3] Hapke, 1986, Icarus 67, 264-280. [4] Hapke et al., Icarus 133, 89-97. [5] Buratti and Veverka, 1984, Icarus 58, 254-264. [6] Verbiscer and Veverka, 1992, Icarus 99, 63-69. [7] Verbiscer and Veverka, 1994, Icarus 110, 155-164. [8] Buratti et al., 1998, Icarus 136, 223-231. [9] Verbiscer and Veverka, 1989, Icarus $82,336-353$ 

END MEMBER ANALYSES OF SPACEBORNE THERMAL INFRARED DATA OF METEOR CRATER, ARIZONA AND APPLICATION TO FUTURE MARS DATA SETS S. P. Wright and M. S. Ramsey, Image Visualization and Infrared Spectroscopy (IVIS) Laboratory [http://ivis.eps.pitt.edu], Department of Geology and Planetary Science, University of Pittsburgh, Pittsburgh, PA 15260-3332, ShawnWright@pittsburghpanthers.com.

Introduction: Thermal infrared data from the Advanced Spaceborne and Thermal Enission and Reflection Radiometer (ASTER) is used to identify the distribution of three lithologies within the ejecta blanket of Meteor Crater, Arizona. This is possible because thermal infrared (TIR) data have been shown to add linearly and thus be easily interpretable [1]. As all small, simple impact craters are initıally created with the same morphology, this has many applications to new data from the Thermal Emission Imaging System (THEMIS) currently in orbit at Mars and will use the premise of Ramsey [2] conceming the use of terrestrial multispectral images as proxies for thermal infrared data sets of Mars.

Background: Non-degraded, sunall impact craters outnumber large craters on unmodified planetary surfaces and are of younger age. Younger impact craters will have less erosion, a well-preserved ejecta blanket, and less dust at the surface, making them excellent sites for thermal infrared (TIR) observations of the surface composition. Of further interest is heing able to identify inner-crater stratigraphy and the distribution of those units in the ejecta blanket in order to infer the geology and local climatic conditions of the region before and after crater formation.

ASTER TIR images are used as a proxy for upcoming THEMIS data by extracting enissivity spectra of the lithologic units representing the Meteor Crater rim and ejecta blanket. ASTER TIR has a comparable spectral resolution and spatial resolution ( 90 meters) to that of THEMIS TIR (100 meters). In order to test methodologies to be used with THEMIS data, image end member deconvolutions are performed using ASTER TIR spectra of the Meteor Crater ejecta blanket. Additionally, hand samples will be collected during field work (summer 2002) and the emission spectra of each lithology will be used as sample end members. The sample end member analysis will be compared to the image end member analysis to validate the image end member analysis.

It should be noted that any conparisons between Meteor Crater and a martian impact crater will be strictly a comparison conceming morphology and the geologic process of impact cratering. The sedimentary lithologies found at Meteor Crater are not likely to be found on the surface of Mars and therefore not in any martian crater or ejecta blanket. However, as all small, simple impact craters are initially created with the same morphology, martian impact craters should show a similar pattern of the distribution of ejecta, which will be deposited in inverse stratigraphic order with respect to the pre-impact stratigraphy. An objective of the project is to attempt to quantify this relationship for select small, simple impact craters. In addition to stratigraphy, other crater properties of Meteor Crater will be investigated to determine if these properties can be identified by TIR remote sensing.

It has been shown that an image end member deconvolution using the thermal infrared wavelengths can be used to identify the lithologies distributed in the Meteor Crater ejecta blanket [3]. However, the airborne Thermal Infrared Multispectral Scanner (TIMS) and its 4 meter spatial resolution was used in the earlier study. Still in question and what this project seeks to answer is if THEMIS data sets, with 100 meter spatial resolution, can be used to identify the lithologic members of small, simple impact craters on Mars. ASTER, rather than TIMS, will be used in this study due to the comparable spatial resolution to THEMIS over the TIR wavelengths. The ASTER image end member analyses will be compared to the earlier TIMS image end member deconvolution for validation.

Study Site: Meteor Crater, Arizona is located in semi-arid north-central Arizona and is estimated to be 49,000 to 50,000 years old, making it one of the most recent and well-preserved impact sites on Earth [4]. The simple, bowl-shaped crater is 180 meters deep and $1.2 \mathrm{~km}$ in diameter, with an eroded rim standing 30-60 meters high [5]. The local geology consists of three flat-lying sedimentary members with known lithologies and contrasting spectral signatures. The oldest unit sampled by impact is the Permian Coconino Sandstone. Above the Coconino are the Permian Kaibab Limestone and a thin veneer of the Triassic Moenkopi Formation. The crater rim and ejecta blanket consists of the same lithologies as described above, but inverted due to impact. Within the ejecta blanket, the units are preserved up to $1.2 \mathrm{~km}$ (or 2 crater radii) away. Additionally, the ejecta blanket has a noticeable wind-streak 
to the northeast due to climatic conditions since crater formation.

Meteor Crater has been described as a perfect example of a well-preserved, simple impact crater by many geologists $[3,4,5,6,7]$. Meteor Crater is convenient to use for remote sensing applications and associated groundtruthing with on-site field research. The amount of previous work, mineralogical contrast between the units [8], low amount of erosion since crater formation $[3,7]$ due to a semi-arid environment and young age, and the mixing of the ejecta make Meteor Crater the logical choice to be an analog for similar craters on the martian surface.

Instrumentation: ASTER is a multispectral imager on the Terra spacecraft and has been operational since October 2000. ASTER has fourteen bands from the visible to the thermal wavelengths, including five in the TIR at 90 meter spatial resolution and three in the visual and near infrared (VNIR) wavelengths at 15 meter resolution [9]. ASTER's TIR bandwidths range from $8.13 \mu \mathrm{m}$ to $11.65 \mu \mathrm{m}$.

THEMIS is a multispectral instrument on the Mars Odyssy orbiter and operates over nine bands from 6.5 $\mu \mathrm{m}$ to $14.5 \mu \mathrm{m}$. THEMIS also has five bands in the VNIR wavelengths at 20 meter resolution [10]. The release of a portion of THEMIS data representing a small $(3 \%-4 \%)$ fraction of the martian surface is tentatively set for October 2002 .

Conclusion and Application to Mars: The results of the study will lead to a greater understanding of THEMIS TIR data of young, small, simple impact craters.

THEMIS TIR data sets of the martian surface will yield the emissivity of each pixel at the highest spatial resolution ever collected of the martian surface [10]. Because a sample end member analysis cannot be performed, the limits of an image end member analysis must be known. An image end member analysis could reveal the distribution of various lithologies within the ejecta blanket and mineral spectral libraries can be used to extract the mineralogical content of each lithology. Impact craters on Mars will be chosen to be examined using THEMIS TIR data based on 1) age, as young impact craters will have less erosion and dust, 2) size and shape, as this study concerns what can be learned from the study of small $(<3-4 \mathrm{~km}$ diameter), simple craters, and 3) the TIR spectra, as the impacted area requires spectrally different stratigraphy.
References: [1] Ramsey and Christensen, JGR 103, pp 577-596, 1998. [2] Ramsey, Mars Infrared Spectroscopy Workshop (ext. abstract), pp 2016, 2002. [3] Ramsey, JGR (in press), 2002. [4] Shoemaker, et al., Guidebook to the Geology of Meteor Crater, Arizona, 1974. [5] Roddy, LPSC IX, pp 3891-3930, 1978. [6] Garvin, et a]., LPSC XX (abstract), pp 333-334, 1989. [7] Grant and Schultz, JGR, 98, pp 15033-15048, 1993. [8] Ramsey and Christensen. TIMS Workshop, JPL Publ. 92-14 (ext. abstract), pp 34-36, 1992. [9] Abrams, Int. Jour. of Rem. Sens., Vol. 21, pp 847-859, 2000. [10] Christensen, et al., Mars Mission Workshop, pp $16-18,1999$. 
AN AUTOMATED SPECTRAL DECONVOLUTION ALGORITHM: APPLICATION TO THERMAL INFRARED STUDIES OF EARTH AND MARS. N. V. Zorn' and M. S. Ramsey ${ }^{2}$, 'Department of Electrical Engineering, University of Pittsburgl, 348 Benedum Hall, Pittsburgh, PA 15261, nvzst@pitt.edu, ${ }^{2}$ Department of Geology and Planetary Science, University of Pittsburgh, 200 SRCC Building, Pittsburgh, PA 15260, ramsey@ivis.eps.pitt.edu.

Introduction: The linear mixing of thermal infrared (TIR) emission spectra in multi-mineralic mixtures has been proven, and its limits and applicability have been quantitatively investigated $[1,2$. Limiting factors in the accuracy of any linear retrieval (spectral deconvolution) algorithm include the spectral precision of the instrumentation as well as the fat that the number of end-members must be $\leq$ the number of spectral bands in the TIR [1]. Because of this end-member constraint, there is no way to examine a multispectral TIR image using a large, spectral end-member library [3]. A possible solution is the implementation of an automated, blind end-member algorithm is analyze all possible subsets (of arbitrary size $k$ ) of minerals present in the mixture from within a mineral library (of size $n$ ). For example, in the only such study to employ this technique, the Kelso Dunes, California were examined using 375 unique combinations $(k 4)$ of the most likely $(n=) 15$ minerals present in the dunes [4]. The deconvolution model results were analyzed for their "goodness of fit" to laboratory spectra of collected sand samples. This investigation proved highly successful, further supporting the capabilities of linear retrieval in the TIR [4]. However, the process was computationally intensive because it was entirely manual. The development of an automated algorithm 11" accomplish this task would decrease the amount of time required for such an investigation by many orders of magnitude.

From a remote sensing perspective, an automated blind end-member spectral deconvolution algorithm could be useful to determine mineral abundance for any mixture (or pixel-by-pixel in a TIR image) using a large emissivity library of minerals as input. This approach is particularly timely as there multispectral TIR mapping instruments now orbiting Eirth and Mars.

Algorithm: Combinatorial mathematics states that the number of unique $k$-subsets of $n$ elements is given by the equation:

$$
n \mathrm{C} k=\frac{n !}{(n-k) ! k !}
$$

It is observed that as $n$ increases for any given $k$, the result grows significantly. For example, $15 \mathrm{C} 4=1,365$ and $20 \mathrm{C} 4=4,845$. Algorithms have been written by combinatorial mathematicians to generate lists of these combinations with different ordering methods. In the techniques developed here, a lexicographic (integer) ordering algorithm was implemented [5].

All routines were written in the MATLAB ${ }^{\circledast}$ environment (Version 6.1.0.450). This software was chosen due to its ability to efficiently manipulate large matrices. The algorithm accepts the following as input: (1) an "unknown" mixture spectrum, at high spectral resolution (518 data points); (2) a library composed of $n$ individual mineral spectra, also at high resolution and possibly present in the unknown; (3) a user-selected maximum output subset size $k$; and (4) a user-selected threshold abundance below which minerals should not appear in the modeled output (e.g. 0-5\%). Concerning (4), the mathematical techniques utilized in the linear deconvolution (and explained in detail in [1]) involve a linear regression analysis that can result in negative values in the output matrix of end-member fractions. Because negative abundances are physically impossible, model results containing these are removed from further consideration. Linear deconvolution can also produce spurious results below the realistic accuracy of the model $(-5 \%)[1]$.

A combinations list is then generated for $n \mathrm{C} 2$, and each unique combination is used as indices for the modeled reconstruction of the unknown emissivity. For example, where $n=20$, the 190 resultant combinations $\{1,2\},\{1,3\},\{1,4\}, \ldots\{18,20\}$ and $\{19,20\}$ are each used to construct a narrowed end-member library, and linear deconvolution is performed on this narrowed library, resulting in a vector of abundance fractions for each combination. If any fraction is less than the low threshold parameter, the combination is removed. Then a candidate emissivity is constructed using each fraction vector, and the root-mean-squared (RMS) error (or "goodness of fit") of the candidate is calculated $[1,4]$. The combination resulting in the lowest RMS error is considered the best fitting combination and stored. Next, the subset size is increased by one, and a new combination list is generated ( $n$ C 3 long). If a betterfitting combination of three elements is found within this list, that combination becomes the new best fit. This process is repeated until $(k)$ is reached. Theoretically, the combination that results in the lowest RMS error will be the one that actually comprises the mixture. 
Preliminary Results: Thermal emission spectra of several mixtures of known mineralic composition were used to initially test the capabilities of this algorithm. The mixtures were composed of hornblende, microcline, oligoclase and quartz, and have been analyzed in prior investigations of spectral deconvolution [1].

Is RMS error sufficient? To conserve processor time and rigorously test the algorithm, a blind endmember library of size $n=20$ was initially used, in conjunction with the TES spectral library of 170 minerals [3]. An end-member library subset was constructed of 16 arbitrarily selected mineral spectra and the four known end-members. The $k$ value was set at $\mathbf{4}$ for each execution, and the low threshold parameter was set at $0 \%$. Therefore, the number of combinations examined was $(20 \mathrm{C} 2)+(20 \mathrm{C} \mathrm{3})+(20 \mathrm{C} 4)=6,175$. The results for three mixtures are tabulated in Table 1 .

\begin{tabular}{|c|c|c|c|c|c|c|}
\hline Mixture & \multicolumn{2}{|c|}{2} & \multicolumn{2}{|c|}{3} & \multicolumn{2}{|c|}{4} \\
\hline Mineral & $\frac{\text { Real }}{\%}$ & Mod. \% & $\frac{\text { Real }}{\%}$ & Mod. \% & $\frac{\text { Real }}{\%}$ & Mod.\% \\
\hline Hornblende & 10 & 0.0 & 20 & 22.4 & 70 & 74.4 \\
\hline Microcline & 10 & 12.9 & 20 & 20.5 & 5 & 9.1 \\
\hline Oligoclase & 20 & 20.3 & 20 & 27.8 & 5 & 0.0 \\
\hline Quartz & 60 & 53.0 & 40 & 30.2 & 20 & 15.6 \\
\hline Pyroxmangite & 0 & 14.4 & 0 & 0.0 & 0 & 0.0 \\
\hline Biotite & 0 & 0.0 & 0 & 0.0 & 0 & 1.6 \\
\hline
\end{tabular}

Table 1. Algorithm output for three mixtures using high-resolution thermal emission spectra.

The modeled mixtures above exhibited the lowest RMS error, and were selected as the best-fitting combinations. The RMS errors for modeled mixtures 2, 3 and 4 were $0.0040,0.0039$ and 0.0025 , respectively. The algorithm at times finds other minerals that compose a mathematically better fit to the unknown than the actual combination of minerals. This suggests that RMS error alone may not be capable of judging absolute "goodness of fit." Also, it is observed that some minerals with abundance $\leq 15 \%$ are not always detected, or other minerals are detected in their place. This was also noted in previous investigations and appears to depend not only on abundance, but on spectral features as well $[1,2]$. These and other characteristics of the algorithm are currently being studied in greater detail.

Algorithm runtime considerations. Performing thousands of spectral deconvolutions per unknown spectrum clearly requires a fast and capable computer. The trials performed in this study were done on a Windows 2000 machine with a $1400 \mathrm{MHz}$ Intel ${ }^{\text {(i) }}$ Pentium $^{\circledR} 4 \mathrm{CPU}$ and $128 \mathrm{MB}$ RAM. The total runtime of the algorithm was measured for end-member libraries of different sizes, ranging from 4 to 50 , and $k=4$. In the
20 end-member library trials $(6,175$ total combinations) the algorithm ran for $\sim 8$ seconds. In a 50 endmember library trial $(251,125$ total combinations) the algorithm ran for $\sim 4,032$ seconds ( 67 minutes). Further investigation into the effects of increasing the number of end-members in the spectral library is underway. Ideally, the algorithm should be able to choose from the entire library of recorded thermal emission spectra and quickly produce a resultant fit.

Effects of degraded spectral resolution. The effect of degrading the spectral resolution of the mineral and mixture spectra is also of interest, given that the Marsorbiting Thermal Emission Imaging System (THEMIS) instrument only has 10 spectral bands in the TIR $(6.5$ and $14.5 \mu \mathrm{m}$ ). The capabilities of a linear retrieval algorithm at this resolution were tested by degrading the spectral resolution of the end-member and mixture spectra to the approximated THEMIS band centers. The resulting fractional abundances are tabulated in Table 2 (compare to the actual compositions of mixtures $2-4$ in Table 1). The RMS errors for modeled mixtures 2, 3 and 4 were $0.0041,0.0033$ and 0.0016, respectively. Further analysis of the accuracy at low spectral resolution is also underway. If successful, this algorithm will be implemented for imaging instruments such as THEMIS in order to identify mineral percentages and track sediment transport over time on the surface of Mars and Earth [3].

\begin{tabular}{|c|c|c|c|}
\hline Mixture & 2 & 3 & 4 \\
\hline Mineral & Mod.\% & Mod.\% & Mod. \% \\
\hline Hormblende & 5.4 & 21.5 & 70.0 \\
\hline Micracline & 10.3 & 19.3 & 18.4 \\
\hline Oligoclase & 32.3 & 29.2 & 0.0 \\
\hline Quartz & 52.9 & 30.7 & 11.9 \\
\hline
\end{tabular}

Table 2. Spectral deconvolution results using 10-band thermal emission spectra.

References: [1] Ramsey M. S. and Christensen P. R. (1998) JGR, 103, 577-596. [2] Feely, K.C. and Christensen P. R. (1999) JGR, 104, 24,195-24,210. [3] Christensen P. R., et al. (2000) JGR, 105, 97359739. [4] Ramsey M. S., et al. (1999) GSA Bulletin, 111, 646-662. [5] Kreher D. L. and Stinson D. R. (1999) Combinatorial algorithms: generation, enumeration, and search, Boca Raton, CRC Press, 43. 
NEGATIVE POLARIZATION OF LIGHT SCATTERED BY COMETARY DUST AND PLANETARY REGOLITH: TWO DIFFERENT MECHANISMS. E. S. Zubko and Yu. G. Shkuratov, Kharkov Astronomical Observatory, 35 Sumskaya St., Kharkov, 61022, Ukraine. zubko@astron.kharkov.ua.

Introduction: The planetary reguliths and dust of comets exhibit negative polarization branch (NPB) of light scattered at small phase angles. This is demonstrated in Figs. 1 and 2, where data for the 1P/Halley comet (dark points in Fig. 1) [1] and asteroid 1 Ceres (opened circles in Fig. 2) [2] are presented. One can see good resemblance of NPB for such different ob-

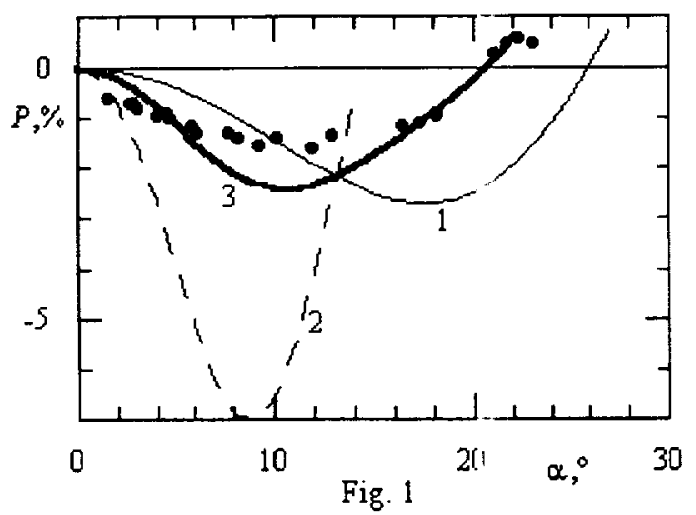

jects; in both cases the NPB have a parabolic shape, and close values of the inversion angle (near $20^{\circ}$ ) and depth (near 1\%). Thus it is reasonahle to suppose that this resemblance can evidence a common origin of the dust and regolith NPB. Nevertheles; here we demon-

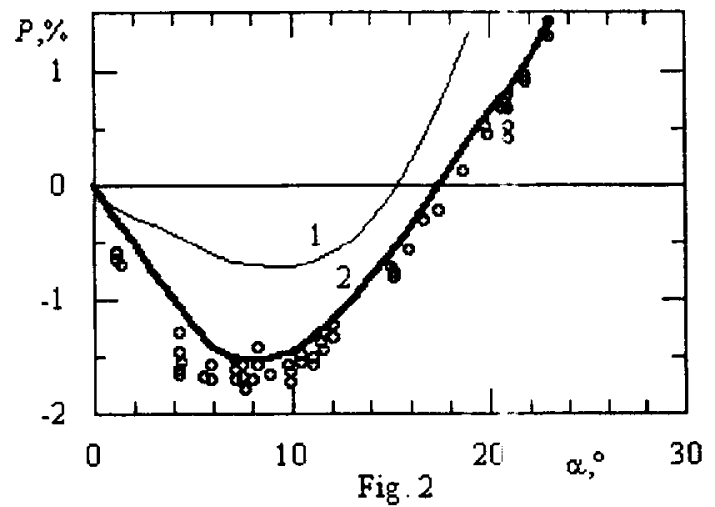

strate that the mechanisms of NPB for comets and planetary surfaces can be quite different. In case of comets this can be due to scattering by individual particles, and for planetary regoliths this could be a combination of the "comet" mechanisn and the coherent backscatter effect.

Light scattering by cometary dust: Individual particles with size compared to the wavelength can in principle produce NPB. This is well known from calcu- lations of scattering properties of spheres and irregular particles by, respectively, the Mie formulas [3] and DDA approach [4]. Spheres as a model of cometary particles are a too rough approximation, since perfect balls, even after particle size averaging, can demonstrate resonant features (both in intensity and in polarization) that are not observed for comets. Results of DDA modeling for irregular particles are rather contradictory. In particular, results obtained in [4] and with the well-known code [5] show bad coincidence. That is why we start DDA modeling using our own codes [6].

Here we simulate scattering properties of dust particles regarding them to scatter independently of each other. Besides we consider that they are randomly shaped and have the following mass distribution: $m^{-u}$, where $u=1.5[7]$ at a constant density of particles. To calculate scattering properties we used an improved DDA method presented in [6]. To simulate cometary dust particles, a set of 137062 cells is used to form an approximately spherical volume. Then two randomly chosen cells of the volume are marked as seeds of a particle material and two cells are considered as seeds of the empty space. Each cell different from the seed cells is marked as the nearest seed cell. Examples of cross-sections of particles generated are shown on Fig. 3. As can be seen we generate particles with very irregular shapes that are undoubtedly closer to real
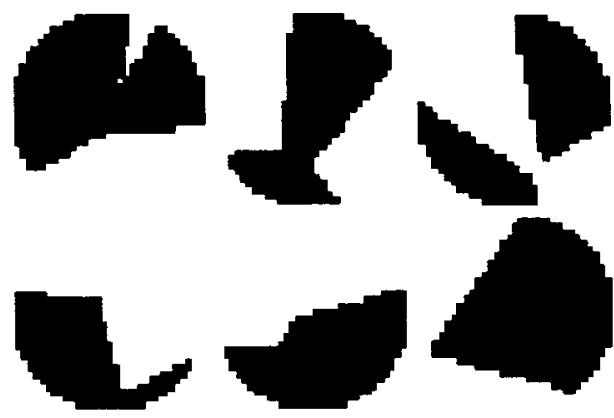

Fig. 3

particles of comets than perfect spheres.

Phase curves of linear polarization degree for a cloud consisting of particles with random shapes are given in Fig. 1. In this figure curve 1 presents data for particles with $m=1.65+0.05 i$ at averaging over sizes from $x=0.1$ to $5.5(x=2 \pi r i \lambda$, where $\lambda$ is the wavelength and $r$ is the radius of the sphere with the volume that is equal to the particle volume). The chosen refractive index can simulate silicate materials with additions 
of carbon. Curve 2 in Fig. 1 corresponds to dirty icy particles $(m=1.31+0.01 i)$; this curve is obtained at averaging over $x=0.1-10.5$. As one can see the curves 1 and 2 are strongly distinct from the 1P/Halley comet data. However, the mixture that is $33 \%$ of silicate and $67 \%$ of icy particles produces curve 3 in Fig. 1, which is a good model of the comet $1 \mathrm{P} /$ Halley NPB. The mixture reproduces the parabolic shape of the NPB, gives almost the same inversion angle and rather close value of the NPB depth. The difference of values of the latter parameter is perhaps due to that we did not take into account in our modeling the contribution of the gas component of the IP/Halley coma.

Thus, NPB of comets can be caused with single particle scattering by rather small particles $(x<10)$, size and compositional distribution of which give the observed parabolic shape of NPB. This is in consistence with recent laboratory investigations [8], where it was shown that clouds of silicate particles of random shapes with size of order of the wavelength do give symmetric NPB with the depth near $2 \%$ and inversion angle of about $20^{\circ}$. At the same time these experiments did not show brightness opposition effect that often accompanies NPB.

Our calculations reveal that despite small number of large particles with $5<x<10$, their contribution to the NPB is rather high. In particular, NPB of symmetric shape can be obtained for two component mixtures of particles with different sizes, even if each component has significantly asymmetric NPB (see Fig. 1).

Light scattering by regoliths: The planetary regoliths, for instance the lunar soils, exhibit conspicuous NPB that are studied in many works. There have been considered many mechanisms of the regolith NPB but the coherent backscatter effect is perhaps the most prospective among them [9]. Even for dark surfaces this mechanism is valid [10]. We model here the NPB of asteroid 1 Ceres, which is a rather dark object.

We treat the planetary regoliths as a particulate media consisting of particles large compared to the wavelength. To calculate scattering properties we used an approach presented in [11]. This approach allows calculations of multiple scattering in a particulate medium with accounting for the coherent backscatter enhancement and shadow-hiding effect.

We consider that the particles of modeling medium have the same size and are randomly shaped, like it is shown in Fig. 3. They are characterized by $m=1.5+$ $0.1 i, x=2$, and single particle albedo $\omega=0.66$. Note that such a particle being isolated does not produce NPB at all. Fig. 2 shows results of our simulation for the particulate medium with volume density $\rho=0.3$. Curves 1 and 2 in this figure present sums of two and six orders of scattering, respectively. As can be seen, the NPB of the medium in the approximation of sixth order is in a good agreement with corresponding data for asteroid Ceres 1 . This example clearly shows that NPB is effectively contributed not only by the second scattering order but high orders too.

We emphasize again that the NPB in this specific case is formed only due to the coherent backscatter effect and definitely not due to polarization produced by individual particles. On the other hand, for planetary regoliths the two considered mechanisms can be important. They are able even to give NPB of complicated forms, in particular, with double minimum. Such NPB were recently discovered for bright asteroids and bright planetary satellites [12] and found in laboratory measurements [13].

Conclusion: Thus, NPB of dust cometary comas and planetary regoliths can generally be given rise by two different mechanisms. One of them is related to single particle scattering. This can be the main cause of comets NPB. We find symmetric (parabolic-like) NPB to be formed due to superposition of very asymmetric NPB corresponding to irregular particles with different sizes. The other mechanism is a collective electromagnetic effect called the coherent backscatter enhancement. This mechanism can form NPB independently of the fact whether the particles have own NPB or not. Unlike the case of scattering by individual particles, the coherent backscattering produce also the prominent brightness opposition effect. We note that cometary comas have actually the brightness opposition surges much less than that for planetary regolith.

References: [1] Kiselev N. N., and Chernova G. P. (1981) Icarus, 48, 473-481. [2] Zellner B. and Gradie J. (1976) Astron. J., 81, 262-280. [3] Brooke T. Y. et al. (1987) Astron. \& Astrophys., 187, 621-624. [4] Lumme K. and Rahola J. (1994) Astrophys. J., 425, 653-667. [5] Draine B. T. and Flatau P. J. (1994) J. Opt. Soc. Am. A., 11, 1491-1499. [6] Zubko E. S. et al. (1999) Solar System Res., 33, 296-301. [7] Mazets E. P. et. al. (1986) Nature, 321, 276-278. [8] Munoz O. et al. (2001) JGR $D, \quad 106, \quad 22,833-22,896$. [9] Shkuratov Yu. et al. (1994) Earth, Moon, and Planets, 65, 201-244. [10] Shkuratov Yu., et al. (2000) Kinematics and Physics of Celestial Bodies. Suppl. Ser., 3. 209-212. [11] Zubko E. S. et al. (2002) Optics and Spectr., 92, 443-448. [12] Rosenbush V. et al. (2002) Icarus (in press). [13] Shkuratov Yu. G. and Ovcharenko A. A. (2002) Workshop on the Optics of Cosmic Dust / Eds. G. Videen and M. Kocifaj (in press). 